\title{
Postfix synchronization methods for OFDM and MIMO-OFDM systems
}

\author{
by \\ Yaobin Wen
}

A thesis submitted to the Faculty of Graduate Studies and Research in partial fulfillment to the requirements for the degree of

Master of Applied Science

Ottawa-Carleton Institute for Electrical and Computer Engineering Department of Systems \& Computer Engineering Carleton University

Ottawa, Ontario, Canada

August 2007

(c) 2007 Yaobin Wen 


$\begin{array}{ll}\begin{array}{l}\text { Library and } \\ \text { Archives Canada }\end{array} & \begin{array}{l}\text { Bibliothèque et } \\ \text { Archives Canada }\end{array} \\ \begin{array}{l}\text { Published Heritage } \\ \text { Branch }\end{array} & \begin{array}{l}\text { Direction du } \\ \text { Patrimoine de l'édition }\end{array} \\ \begin{array}{l}\text { 395 Wellington Street } \\ \text { Ottawa ON K1A ON4 }\end{array} & \begin{array}{l}\text { 395, rue Wellington } \\ \text { Ottawa ON K1A ON4 } \\ \text { Canada }\end{array}\end{array}$

Your file Votre référence ISBN: 978-0-494-33673-1 Our file Notre référence ISBN: 978-0-494-33673-1

NOTICE:

The author has granted a nonexclusive license allowing Library and Archives Canada to reproduce, publish, archive, preserve, conserve, communicate to the public by telecommunication or on the Internet, loan, distribute and sell theses worldwide, for commercial or noncommercial purposes, in microform, paper, electronic and/or any other formats.

The author retains copyright ownership and moral rights in this thesis. Neither the thesis nor substantial extracts from it may be printed or otherwise reproduced without the author's permission.
AVIS:

L'auteur a accordé une licence non exclusive permettant à la Bibliothèque et Archives Canada de reproduire, publier, archiver, sauvegarder, conserver, transmettre au public par télécommunication ou par l'Internet, prêter, distribuer et vendre des thèses partout dans le monde, à des fins commerciales ou autres, sur support microforme, papier, électronique et/ou autres formats.

L'auteur conserve la propriété du droit d'auteur et des droits moraux qui protège cette thèse. $\mathrm{Ni}$ la thèse ni des extraits substantiels de celle-ci ne doivent être imprimés ou autrement reproduits sans son autorisation.
In compliance with the Canadian

Privacy Act some supporting forms may have been removed from this thesis.

While these forms may be included in the document page count, their removal does not represent any loss of content from the thesis.
Conformément à la loi canadienne sur la protection de la vie privée, quelques formulaires secondaires ont été enlevés de cette thèse.

Bien que ces formulaires aient inclus dans la pagination, il n'y aura aucun contenu manquant.

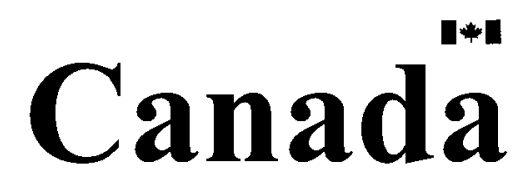




\begin{abstract}
This thesis presents a novel synchronization method for OFDM and Multiple Input Multiple Output (MIMO)-OFDM systems which use preambles containing two identical training parts (Chu sequences) followed by a Flipped Postfix (FIP).

The proposed synchronization method is implemented in four steps: coarse time synchronization, estimation of the Carrier Frequency Offset (CFO) fraction and integer part, and fine time synchronization.

We extend the proposed and conventional Schmidl/Cox (SC) methods to MIMO systems. By using multiple antennas, the spatial diversity improves the accuracy of the symbol timing estimator and CFO estimator.

From the analysis and simulation, the proposed postfix synchronization method has a narrow and large timing metric peak value suitable to specify a threshold and is robust in continuous or burst communication scenarios under different channels with large CFO. It is also robust to the Doppler spread.
\end{abstract}




\section{Acknowledgments}

I would like to express my deep gratitude to my thesis supervisor, Prof. Florence Danilo-Lemoine, for her great help, support, understanding and supervising this research work. Her patience and extensive guidance helped me fulfill this thesis smoothly.

Sincere thanks to the Natural Science and Engineering Research Council(NSERC) of Canada and Prof. Florence Danilo-Lemoine for the financial support during my studies. Without their generosity, my studies could not be completed.

Thanks to Prof. Florence Danilo-Lemoine, Prof. Peter Galko, Prof. Halim Yanikomeroglu, Prof. Richard M. Dansereau, Prof. Sergey Loyka, Prof. Claude D'Amours and Prof. Abbas Yongacoglu, who provided the fundamental knowledge for my research.

Thanks to my colleagues in BCWS lab, Bijan Golkar, Haoming Li and Chaowen $\mathrm{Gu}$, who provided helps during my studies.

Finally, I acknowledge the constant support and encouragement from my family and friends throughout my studies. 


\section{Contents}

1 Introduction 1

1.1 Thesis objectives ......................... 1

1.2 Thesis contributions ................. 5

1.3 Thesis organization ................. 6

2 Background and system model $\quad 8$

2.1 Multipath fading channel . . . . . . . . . . . . 8

2.1.1 Wide-Sense-Stationary with Uncorrelated Scattering (WSSUS)

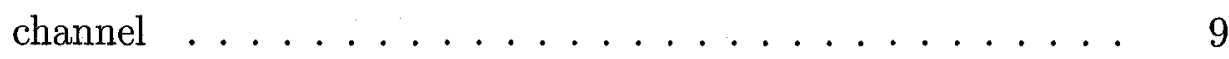

2.1.2 Fast fading vs. slow fading . . . . . . . . . . . . . 12

2.1.3 Flat fading vs. frequency selective fading . . . . . . . . . . 12

2.1.4 A Tapped delay line channel model [17] . . . . . . . . . . . 13

2.2 Representation of OFDM signal . . . . . . . . . . . 15

2.2.1 Transmitted OFDM signal (the simplest version) . . . . . 15

2.2 .2 OFDM digital implementation . . . . . . . . . . 16

2.2 .3 Adding Cyclic Prefix . . . . . . . . . . . . 18

2.2 .4 Adding windowing ................. 20

2.2.5 Transmitted OFDM signal (including CP and windowing) . . 20

2.2.6 Received signal (assuming perfect synchronization) . . . . . 20 
2.2.7 The OFDM system block diagram . . . . . . . . . . 23

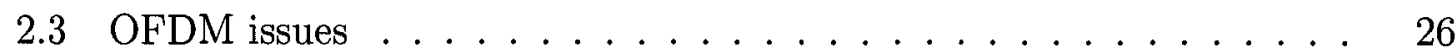

2.3.1 Coding and Modulation .............. 26

2.3.2 Issues related to Cyclic Prefix . . . . . . . . . . . . . 26

2.3.3 The Peak to Average Power Ratio problem . . . . . . . . . . 27

2.3.4 Synchronization of OFDM systems [3] . . . . . . . 27

2.4 MIMO-OFDM systems background . . . . . . . . . . . . . . 30

2.4 .1 MIMO systems ...................... 30

2.4 .2 MIMO-OFDM systems . . . . . . . . . . . 32

2.5 System model . . . . . . . . . . . . . . . . . . 33

2.5 .1 OFDM system model . . . . . . . . . . . . 33

2.5.2 MIMO-OFDM system model . . . . . . . . . . . 34

3 Existing synchronization methods 36

3.1 Synchronization methods using training symbols . . . . . . . . . . 36

3.1.1 Schmidl and Cox $(\mathrm{SC})$ 's method $[6] \ldots \ldots 36$

3.1 .2 Minn's method $[7] \ldots \ldots . \ldots \ldots$

3.2 Synchronization methods for MIMO-OFDM systems . . . . . . . . 41

4 Proposed synchronization method for OFDM and MIMO-OFDM $\begin{array}{ll}\text { systems } & 46\end{array}$

4.1 Proposed synchronization method for OFDM systems . . . . . . . 46

4.1.1 The training symbols structure of the proposed method . . . . 46

4.1.2 Implementing the proposed synchronization method . . . . . . 49

4.1.3 Analysis of effect of the FlP in an AWGN channel . . . . . . . 55

4.2 Proposed synchronization method for MIMO-OFDM systems . . . . 59

4.2.1 Preamble structures for MIMO-OFDM systems . . . . . . . 60 
4.2.2 Implementing the proposed synchronization method for MIMO-

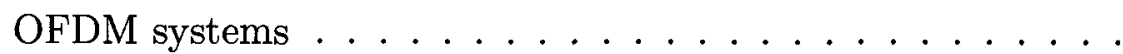

5 Simulation results

68

5.1 Simulation parameters .................... 68

5.2 Simulation results in an AWGN channel . . . . . . . . . . 70

5.3 Simulation results in the first ISI channel . . . . . . . . . . . . 75

5.4 Simulation results in the second ISI channel . . . . . . . . . 80

5.5 Simulation results in the Rayleigh fading channel . . . . . . . . 85

5.5.1 Simulation results in the time-invariant Rayleigh fading channel 85

5.5.2 Simulation results in the time-variant Rayleigh fading channel 89

5.6 Simulation results for MIMO-OFDM systems . . . . . . . . . 101

5.6.1 Simulation results in the time-invariant Rayleigh fading channel for MIMO-OFDM systems . . . . . . . . . . . . . . 101

5.6.2 Simulation results in the time-variant Rayleigh fading channel for MIMO-OFDM systems . . . . . . . . . . . . . 109

6 Conclusions and future works $\quad 118$

6.1 Conclusions ............................. 118

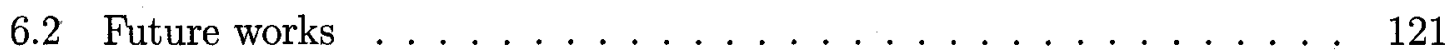

$\begin{array}{ll}\text { Appendices } & 123\end{array}$

A The distribution of $M_{d}=M_{p}\left(d_{o p t}\right)-M_{p}\left(d_{o p t+1}\right)$

B Setting the Threshold for Fine Time Synchronization $\quad 154$

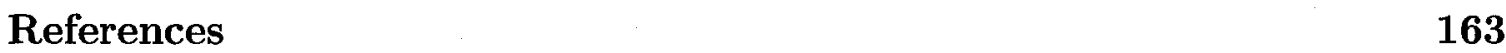




\section{List of Figures}

2.1 The cyclic prefix is a copy of the last part of the present symbol . . 18

2.2 The OFDM system block diagram . . . . . . . . . . . . . 25

2.3 $\mathrm{Q} \times \mathrm{L}$ MIMO-OFDM system, where $\mathrm{Q}$ and $\mathrm{L}$ are the numbers of inputs

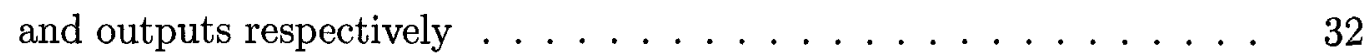

2.4 Block diagram of a system with $\mathrm{Q} \times \mathrm{L}$ transmit-receive diversity . . . $\quad 34$

3.1 Frame structure for the $\mathrm{Q} \times \mathrm{L}$ MIMO-OFDM system . . . . . . 41

4.1 First symbol structure of proposed method and SC's method . . . . . 47

4.2 Comparison of simulated and theoretical $M_{d}$ statistic using SC and

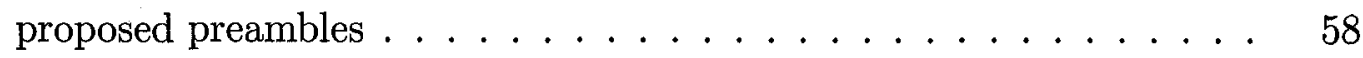

4.3 Timing metric in a noise free ideal channel. . . . . . . . . . . . . 59

5.1 Performance of the timing estimator in an AWGN channel . . . . . 72

5.2 Performance of the CFO (fraction part) estimator in an AWGN channel 73

5.3 Performance of the CFO estimator in an AWGN channel . . . . . . . 74

5.4 Performance of the timing estimator in the first ISI channel . . . . . . 76

5.5 Performance of the CFO (fraction part) estimator in the first ISI channel 78

5.6 Performance of the CFO estimator in the first ISI channel . . . . . 79

5.7 Performance of the timing estimator in the second ISI channel . . . . 82 
5.8 Performance of the CFO (fraction part) estimator in the second ISI

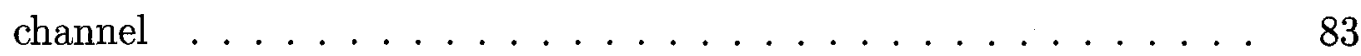

5.9 Performance of the CFO estimator in the second ISI channel . . . . . 84

5.10 Performance of the timing estimator in the time-invariant Rayleigh

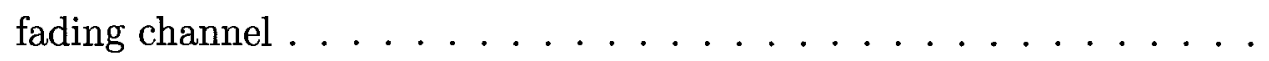

5.11 Performance of the CFO (fraction part) estimator in the time-invariant Rayleigh fading channel ................. 87

5.12 Performance of the CFO estimator in the time-invariant Rayleigh fad-

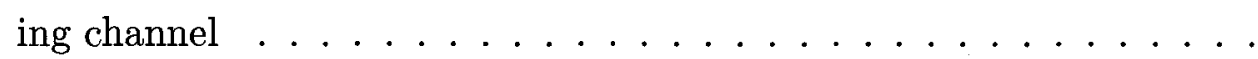

5.13 Performance of the timing estimator in the time-variant Rayleigh fading channel, $v=10 \mathrm{~km} / \mathrm{hr}, f_{m}=46.3 \mathrm{H}_{z} \ldots \ldots \ldots$

5.14 Performance of the CFO (fraction part) estimator in the time-variant Rayleigh fading channel, $v=10 \mathrm{~km} / \mathrm{hr}, f_{m}=46.3 H_{z} \ldots \ldots$

5.15 Performance of the CFO estimator in the time-variant Rayleigh fading channel, $v=10 \mathrm{~km} / \mathrm{hr}, f_{m}=46.3 H_{z} \ldots \ldots \ldots$

5.16 Performance of the timing estimator in the time-variant Rayleigh fading channel, $v=70 \mathrm{~km} / \mathrm{hr}, f_{m}=324.1 H_{z} \ldots \ldots \ldots$

5.17 Performance of the CFO (fraction part) estimator in the time-variant Rayleigh fading channel, $v=70 \mathrm{~km} / \mathrm{hr}, f_{m}=324.1 H_{z} \ldots \ldots$

5.18 Performance of the CFO estimator in the time-variant Rayleigh fading channel, $v=70 \mathrm{~km} / \mathrm{hr}, f_{m}=324.1 H_{z} \ldots \ldots \ldots$

5.19 Performance of the timing estimator in the time-variant Rayleigh fading channel, $v=250 \mathrm{~km} / \mathrm{hr}, f_{m}=1157.4 H_{z} \ldots \ldots 96$

5.20 Performance of the CFO (fraction part) estimator in the time-variant Rayleigh fading channel, $v=250 \mathrm{~km} / \mathrm{hr}, f_{m}=1157.4 H_{z} \ldots \ldots$ 
5.21 Performance of the CFO estimator in the time-variant Rayleigh fading channel, $v=250 \mathrm{~km} / \mathrm{hr}, f_{m}=1157.4 H_{z} \ldots \ldots \ldots$

5.22 Performance of the timing estimator in the time-variant Rayleigh fading channel for various maximum Doppler frequencies . . . . . . . . .

5.23 Comparing the performance of the CFO estimator in the time-variant Rayleigh fading channel for various maximum Doppler frequencies . .

5.24 Performance of the timing estimator in the time-invariant Rayleigh fading channel, $Q=2, L=2 \ldots \ldots \ldots$. . . . . . . . 104

5.25 Performance of the CFO estimator in the time-invariant Rayleigh fading channel, $Q=2, L=2 \ldots \ldots \ldots$. . . . . . . . . . 105

5.26 Performance of the proposed coarse timing estimator in the time-invariant Rayleigh fading channel using different number of antennas . . . . . 106

5.27 Performance of the proposed fine timing estimator in the time-invariant Rayleigh fading channel using different number of antennas . . . . . . 107

5.28 Performance of the proposed CFO estimator in the time-invariant Rayleigh fading channel using different number of antennas . . . . . . . . 108

5.29 Performance of the timing estimator in the time-variant Rayleigh fading channel, $Q=2, L=2, v=10 \mathrm{~km} / \mathrm{hr}, f_{m}=46.3 H_{z} \ldots \ldots 110$

5.30 Performance of the CFO estimator in the time-variant Rayleigh fading channel, $Q=2, L=2, v=10 \mathrm{~km} / \mathrm{hr}, f_{m}=46.3 H_{z} \ldots \ldots 11$

5.31 Performance of the timing estimator in the time-variant Rayleigh fading channel, $Q=2, L=2, v=70 \mathrm{~km} / \mathrm{hr}, f_{m}=324.1 H_{z} \ldots \ldots$

5.32 Performance of the CFO estimator in the time-variant Rayleigh fading channel, $Q=2, L=2, v=70 \mathrm{~km} / \mathrm{hr}, f_{m}=324.1 H_{z} \ldots \ldots 113$

5.33 Performance of the timing estimator in the time-variant Rayleigh fading channel, $Q=2, L=2, v=250 \mathrm{~km} / \mathrm{hr}, f_{m}=1157.4 H_{z} \ldots \ldots 114$ 
5.34 Performance of the CFO estimator in the time-variant Rayleigh fading channel, $Q=2, L=2, v=250 \mathrm{~km} / \mathrm{hr}, f_{m}=1157.4 H_{z} \ldots \ldots 115$

5.35 Performance of the timing estimator using EGC in the time-variant Rayleigh fading channel for various maximum Doppler frequencies, $Q=$ $2, L=2 \ldots \ldots \ldots \ldots \ldots \ldots$

5.36 Performance of the CFO estimator using EGC in the time-variant Rayleigh fading channel for various maximum Doppler frequencies, $Q=2, L=2 \ldots \ldots \ldots \ldots \ldots$ 


\section{List of Tables}

B.1 The probability of $\psi_{d_{o p t 2}}<\eta_{A W G N}$ and $\psi_{n, n \neq d_{o p t 2}}>\eta_{A W G N}$ for different SNR under AWGN channels . . . . . . . . . . . . . . 157

B.2 The probability of $\psi_{d_{o p t 2}}<\eta_{I S I}$ and $\psi_{n, n<d_{o p t 2}}>\eta_{I S I}$ for different SNR under ISI channels . . . . . . . . . . . . . . . . 159 


\section{List of Abbreviations}

$\begin{array}{ll}\text { 4G } & \text { Fourth Generation } \\ \text { AWGN } & \text { Additive White Gaussian Noise } \\ \text { BER } & \text { Bit Error Rate } \\ \text { CDMA } & \text { Code Division Multiple Access } \\ \text { CFO } & \text { Carrier Frequency Offset } \\ \text { CIR } & \text { Channel Impulse Response } \\ \text { CLT } & \text { Central Limit Theorem } \\ \text { CP } & \text { Cyclic Prefix } \\ \text { DAB } & \text { Digital Audio Broadcasting } \\ \text { DFT } & \text { Discrete Fourier Transform } \\ \text { DQPSK } & \text { Differential Quadrature Phase-Shift Keying } \\ \text { DVB-T } & \text { Digital Video Broadcasting } \\ \text { EGC } & \text { Equal Gain Combining } \\ \text { FCP } & \text { Flipped Cyclic Prefix } \\ \text { FDM } & \text { Frequency Division Multiplexing } \\ \text { FIP } & \text { Flipped Postfix } \\ \text { ICI } & \text { Intercarrier Interference } \\ \text { IDFT } & \text { Inverse Discrete Fourier Transform } \\ \text { IFFT } & \text { Inverse Fast Fourier Transform } \\ \text { ISI } & \text { Intersymbol Interference } \\ & \end{array}$




\begin{tabular}{|c|c|}
\hline LAN & Local Area Network \\
\hline MAN & Metropolitan Area Network \\
\hline MIMO & Multiple-Input Multiple-Output \\
\hline MISO & Multiple-Input Single-Output \\
\hline $\mathrm{MRC}$ & Maximum Ratio Combining \\
\hline OFDM & Orthogonal Frequency Division Multiplexing \\
\hline PAPR & Peak to Average Power Ratio \\
\hline $\mathrm{PN}$ & Pseudo-random Noise \\
\hline QAM & Quadrature amplitude modulation \\
\hline QPSK & Quadrature Phase-Shift Keying \\
\hline RV & Random Variable \\
\hline RVs & Random Variables \\
\hline $\mathrm{SC}$ & Schmidl and Cox \\
\hline SIMO & Single-Input Multiple-Output \\
\hline SISO & Single-Input Single-Output \\
\hline SNR & Signal to Noise Ratio \\
\hline STBC & Space Time Block Codes \\
\hline STTC & Space Time Trellis Codes \\
\hline SVD & Singular Value Decomposition \\
\hline $\mathrm{VCO}$ & Voltage Controlled Oscillator \\
\hline WSSUS & Wide-Sense-Stationary Uncorrelated Scattering \\
\hline $\mathrm{ZPP}$ & Zero Padding Prefix \\
\hline
\end{tabular}




\section{List of Symbols}

page where

symbol

Symbol

Explanation

is defined

$\angle$

Argument of a complex number

38

$a_{q, 0}$

Identical part of the first training symbol from the $q^{\text {th }}$

60

$\boldsymbol{a}_{\boldsymbol{q}, \boldsymbol{k}} \quad$ Circular right shift $k$ samples version of $\boldsymbol{a}_{\boldsymbol{q}, \mathbf{0}} \ldots \ldots \ldots \ldots \ldots \ldots$

Complex conjugation of a matrix or vector $\ldots \ldots \ldots \ldots \ldots . . \ldots 37$

$\boldsymbol{b}_{1,0} \quad$ Second training symbol of the first transmitter antenna ..... 62

$\boldsymbol{b}_{1,(q-1) G} \quad$ Circular right shift $(q-1) G$ samples version of $\boldsymbol{b}_{1,0} \ldots \ldots \ldots .62$

$B_{d} \quad$ Doppler spread of the channel $\ldots \ldots \ldots \ldots \ldots \ldots \ldots \ldots \ldots \ldots \ldots$

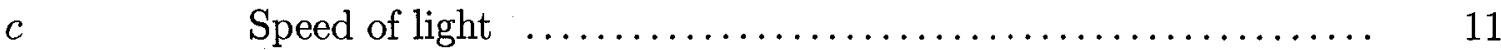

$\circledast \quad$ Convolution operator $\quad \ldots \ldots \ldots \ldots \ldots \ldots \ldots \ldots \ldots \ldots \ldots \ldots \ldots \ldots \ldots \ldots$

$\dagger \quad$ Hermitian conjugation of a matrix or a vector $\ldots \ldots \ldots \ldots \ldots .61$

$\delta_{f} \quad$ Carrier frequency offset $\ldots \ldots \ldots \ldots \ldots \ldots \ldots \ldots \ldots \ldots \ldots \ldots \ldots \ldots \ldots \ldots \ldots$

$\gamma \quad$ Carrier frequency offset normalized by the subcarrier spacing . $\quad 34$

$(\Delta f)_{H} \quad$ Coherence bandwidth of the channel $\ldots \ldots \ldots \ldots \ldots \ldots \ldots .10$

$\delta_{i l} \quad$ Kronecker delta: $\forall i \neq l \delta_{i l}=0$ and $\delta_{l l}=1 \ldots \ldots \ldots \ldots \ldots \ldots$

$\delta(t) \quad$ Dirac delta function $\quad \ldots \ldots \ldots \ldots \ldots \ldots \ldots \ldots \ldots \ldots \ldots \ldots \ldots \ldots \ldots \ldots$ 
$(\Delta t)_{H} \quad$ Coherence time of the channel $\quad \ldots \ldots \ldots \ldots \ldots \ldots \ldots \ldots \ldots . \ldots \ldots$

$d_{o p t}$

Exact symbol start point excluding the prefix and postfix .... 55

$E\{\cdot\}$

Expectation of the argument

$\epsilon$

Symbol time offset 27

$f_{c}$

Carrier frequency 11

$f_{m}$

Maximum Doppler frequency

Fraction part of the carrier frequency offset

Complex amplitude of the $n^{\text {th }}$ path delay

$H(f ; t) \quad$ Fourier transform of $h(\tau ; t)$ respected to $\tau \ldots \ldots \ldots \ldots \ldots \ldots . .10$

$h_{m}$

Complex baseband channel impulse response at lag $m$...... $\quad 34$

$h_{n}(t)$

$n^{\text {th }}$ tap weight of the tapped delay line channel

$h_{q l, m}$

Complex baseband channel impulse response at lag $m$ between the $q^{\text {th }}$ transmitter antenna and the $l^{\text {th }}$ receiver antenna $\ldots \ldots \ldots \ldots . \quad 35$

$h(\tau) \quad$ Complex baseband time invariant channel impulse response $\quad . . \quad 21$

$h(\tau ; t) \quad$ Complex baseband time variant channel impulse response $\quad \ldots \quad 9$

$\Im\{\cdot\} \quad$ Imaginary part of the argument $\ldots \ldots \ldots \ldots \ldots \ldots \ldots \ldots . \ldots . \ldots . \ldots$

$L$

Number of the receiver antennas $\ldots \ldots \ldots \ldots \ldots \ldots \ldots \ldots . . \ldots \ldots$

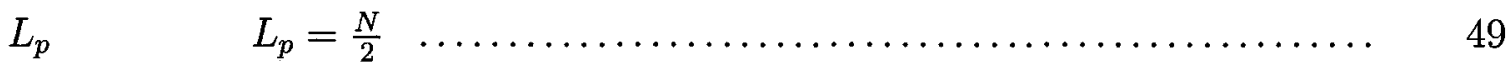

$M \quad$ Length of the tapped delay line channel model $\ldots \ldots \ldots \ldots \ldots . \quad 14$

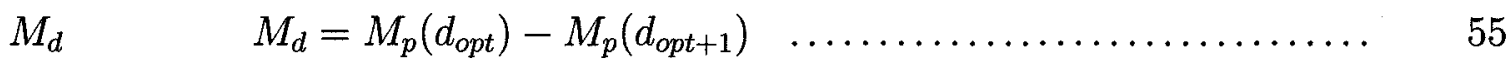

$M_{l}(d) \quad$ Time metric of the proposed method for the $l^{\text {th }}$ receiver

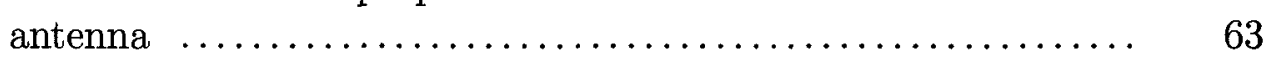

$M_{M I M O}(d) \quad$ Time metric of the proposed method for MIMO-OFDM

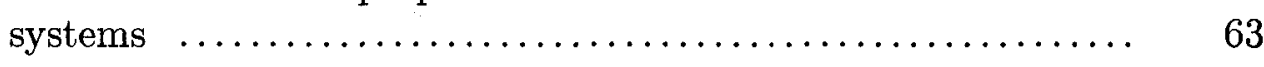

$M_{\text {Minn }}(d) \quad$ Time metric of Minn's method $\ldots \ldots \ldots \ldots \ldots \ldots \ldots \ldots \ldots . . \ldots \ldots$

$\begin{array}{lll}M_{p}(d) \quad \text { Time metric of the proposed method for SISO-OFDM systems } & 49\end{array}$

$M_{S C}(d) \quad$ Time metric of SC's method $\ldots \ldots \ldots \ldots \ldots \ldots \ldots \ldots \ldots . \ldots \ldots$ 
$N \quad$ Total number of subcarriers $\ldots \ldots \ldots \ldots \ldots \ldots \ldots \ldots \ldots \ldots \ldots \ldots$

Gumber of the cyclic prefix samples $\quad \ldots \ldots \ldots \ldots \ldots \ldots \ldots \ldots$

$n_{\epsilon} \quad$ An integer representing the unknown arrival time of a symbol $\quad 34$

$n_{k} \quad k^{\text {th }}$ sample of the complex Gaussian noise with zero mean and variance

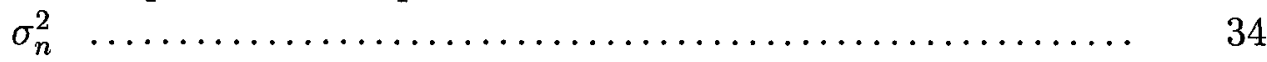

$n_{l, k} \quad k^{\text {th }}$ sample of the complex Gaussian noise with zero mean and variance

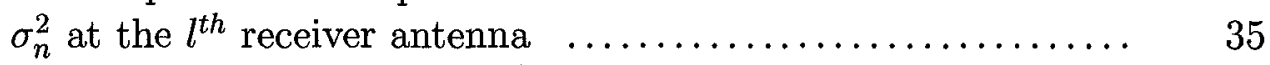

$n(t) \quad$ Additive White Gaussian Noise $\ldots \ldots \ldots \ldots \ldots \ldots \ldots \ldots \ldots \ldots$

$P_{d} \quad P_{d}=\left\|P_{p}\left(d_{o p t}\right)\right\|^{2}-\left\|P_{p}\left(d_{o p t+1}\right)\right\|^{2} \ldots \ldots \ldots \ldots \ldots \ldots \ldots \ldots \ldots \ldots \ldots \ldots \ldots \ldots \ldots \ldots$

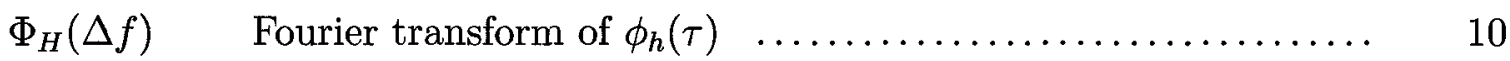

$\phi_{h}(\tau) \quad$ Multipath intensity profile of the channel $\ldots \ldots \ldots \ldots \ldots \ldots . \quad 10$

Q Number of the transmitter antennas $\ldots \ldots \ldots \ldots \ldots \ldots \ldots \ldots$

$r_{k}^{c} \quad$ Carrier frequency corrected time domain sample $\ldots \ldots \ldots \ldots . \quad 53$

$r_{l, k}^{c} \quad k^{t h}$ carrier frequency corrected time domain sample at the $l^{\text {th }}$ receiver

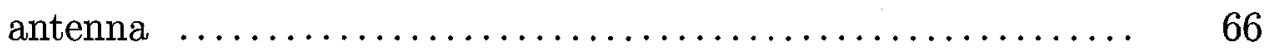

$R_{d} \quad R_{d}=R_{p}^{2}\left(d_{o p t+1}\right)-R_{p}^{2}\left(d_{\text {opt }}\right) \ldots \ldots \ldots \ldots \ldots \ldots \ldots \ldots \ldots \ldots \ldots \ldots \ldots \ldots \ldots \ldots$

$\Re\{\cdot\} \quad$ Real part of the argument $\ldots \ldots \ldots \ldots \ldots \ldots \ldots \ldots \ldots \ldots \ldots \ldots$

$r_{k} \quad k^{t h}$ received sample $\ldots \ldots \ldots \ldots \ldots \ldots \ldots \ldots \ldots \ldots \ldots \ldots \ldots \ldots \ldots \ldots \ldots \ldots \ldots$

$r_{l, k} \quad k^{t h}$ received sample at the $l^{\text {th }}$ receiver antenna $\ldots \ldots \ldots \ldots \ldots .35$

$S_{H}(\lambda) \quad$ Doppler power spectrum of the channel $\ldots \ldots \ldots \ldots \ldots \ldots \ldots . . \ldots$

$S_{H}(\tau ; \lambda) \quad$ Scattering function of the channel $\ldots \ldots \ldots \ldots \ldots \ldots \ldots \ldots \ldots$

$S_{q, k} \quad$ Transmitted data on the $k^{\text {th }}$ subcarrier from the $q^{\text {th }}$ transmitter

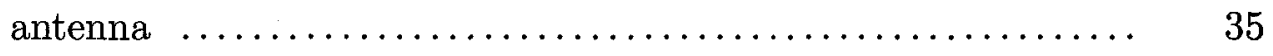

$s_{q, n} \quad n^{t h}$ time domain sample from the $q^{\text {th }}$ transmitter antenna $\ldots \quad 35$

$\tau_{\max } \quad$ Delay spread or maximum channel delay $\ldots \ldots \ldots \ldots \ldots \ldots .21$

$\tau_{n}(t) \quad n^{t h}$ path delay $\ldots \ldots \ldots \ldots \ldots \ldots \ldots \ldots \ldots \ldots \ldots \ldots \ldots \ldots \ldots \ldots \ldots \ldots \ldots \ldots \ldots$

$\tau_{r m s} \quad$ Root-mean-square delay spread $\ldots \ldots \ldots \ldots \ldots \ldots \ldots \ldots \ldots \ldots$ 
$\begin{array}{lll}T_{c p} & \text { Duration of the cyclic prefix } \\ & \ldots \ldots \ldots \ldots \ldots \ldots \ldots \ldots \ldots \ldots \ldots \ldots\end{array}$

$T_{F F T} \quad$ Duration of the OFDM symbol excluding the windowing and

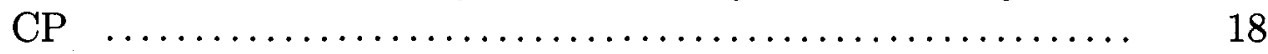

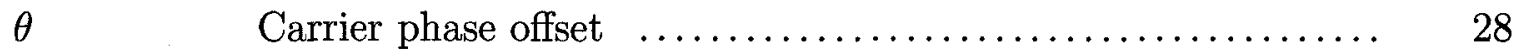

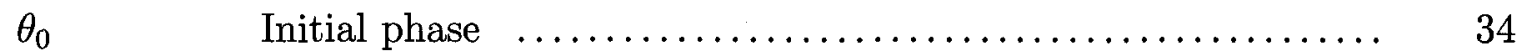

$\tilde{r}(t) \quad$ Lowpass equivalent form of the received signal $\ldots \ldots \ldots \ldots . . .21$

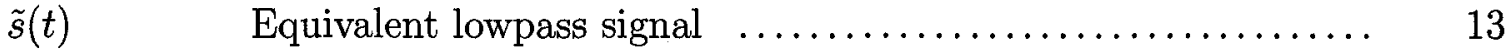

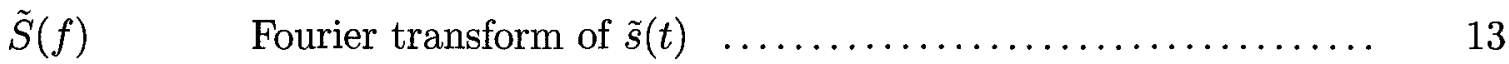

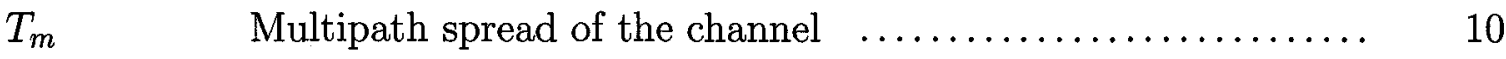

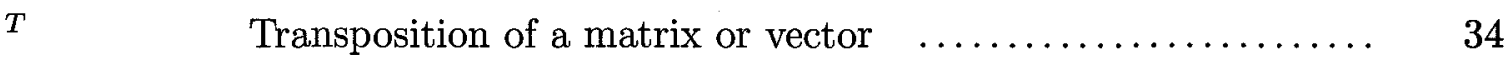

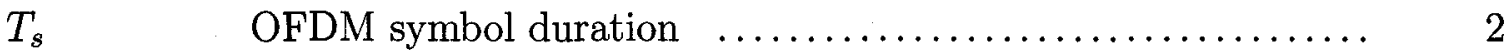

$T_{s a} \quad$ Sample duration $\quad \ldots \ldots \ldots \ldots \ldots \ldots \ldots \ldots \ldots \ldots \ldots \ldots \ldots \ldots \ldots \ldots, \quad 33$

$T_{\text {win }} \quad$ Duration of the windowing (one side) $\ldots \ldots \ldots \ldots \ldots \ldots \ldots . . \ldots$

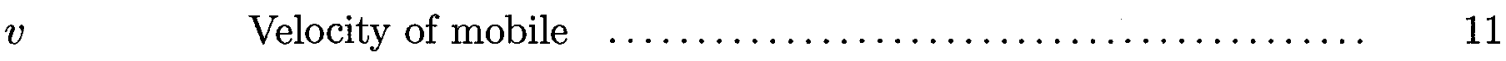

$W \quad$ Bandwidth occupied by the real bandpass signal $\ldots \ldots \ldots \ldots . . . .13$

$W_{s} \quad$ Signal bandwidth excluding the bandwidth occupied by the windowing and $\mathrm{CP}$....................................... 18

$X_{i} \quad$ Complex modulated data on the $i^{t h}$ subcarrier $\ldots \ldots \ldots \ldots . . . . .33$

$x_{n} \quad n^{\text {th }}$ sample of the time domain symbol $\ldots \ldots \ldots \ldots \ldots \ldots \ldots$ 


\section{Chapter 1}

\section{Introduction}

\subsection{Thesis objectives}

Orthogonal frequency division multiplexing (OFDM) is a digital modulation scheme for high data rate wireless transmission. The principle of OFDM is to split a high rate data stream (wide band signal) into a number of subcarriers, i.e. changing a high rate serial data stream into a slow rate parallel data stream. OFDM has been accepted in several wireless standards such as digital audio broadcasting (DAB), digital video broadcasting (DVB-T), the IEEE 802.11a local area network (LAN) standard and the 802.16a metropolitan area network (MAN) standard. It is also a potential candidate for fourth generation (4G) mobile wireless systems.

- Multicarrier vs. Single carrier

A high rate data stream, transmitted serially on a single carrier has a narrow symbol duration, therefore single carrier systems are very sensitive to the delay spread that can cause significant intersymbol interference (ISI) in multipath fading channels. Using multicarrier systems can delimitate the ISI but increases the complexity of the system and sometimes introduces intercarrier interference. 
- Orthogonal vs. Nonorthogonal [1]

In the classic way, a guard band is put between each subcarrier in the frequency domain, which makes the frequency use inefficient. To cope with the spectrum inefficiency, an idea proposed from the mid-1960s was to use parallel data and frequency division multiplexing (FDM) with overlapping subchannels. Each subcarrier is orthogonal with others in each symbol duration, i.e. the carrier space is a multiple of $1 / T_{s}$ (excluding the cyclic prefix and windowing), where $T_{s}$ is the OFDM symbol duration.

- OFDM is robust against narrow band interference [2] Narrow band interference only affects a small fraction of the subcarriers. We can use powerful coding to correct those errors.

- OFDM can use discontinuous bandwidth for operation OFDM is easy to deal with separate band resources and suitable for the wide band demand.

The main advantage of OFDM is that it has a low implementation cost when it transmits over a high frequency selective channel, but it suffers from two drawbacks. One is the peak to average power ratio (PAPR) problem due to the coherent summation of the sine waves, the other is sensitivity to synchronization errors [3]. Synchronization errors such as symbol time error and carrier frequency offset (CFO) cause intersymbol interference (ISI) and intercarrier interference (ICI). Therefore, designing a reliable synchronization scheme is very important for OFDM systems. There are several approaches for the OFDM synchronization: detecting the power of the arriving signal, using the cyclic prefix and using the training symbols.

In [4], the power of the arriving signal is used to find the beginning of the OFDM frame. It did not work well in fading channels and did not consider the CFO estima- 
tion.

In [5], redundant information contained within the Cyclic Prefix (CP) is used to perform joint maximum likelihood symbol time and CFO estimation but reliability is low in multipath fading channels since the CP is short and corrupted by the previous symbol.

Using the training symbols can much more improve timing estimation but the overhead causes inefficiency. Schmidl and Cox (SC) use a training symbol with two identical halves in the time domain to find the start point of the symbol and the fraction part of the CFO. Then they use a second symbol containing differential modulated Pseudo-random Noise (PN) sequence information to estimate the integer part of the CFO [6]. However, due to the CP, there is an inherent plateau in the timing metric that leads to some uncertainty as to the start of the frame. To avoid this effect, the timing estimator is taken as the average of the left and right points at $90 \%$ peak value. SC's method is robust but the timing estimator has a large variance and its mean value is shift to the left by a significant amount. To avoid the occurrence of the plateau and hence some of the uncertainty, various training patterns have been subsequently proposed instead of the two identical halves (e.g. $[A A-A-A]$ proposed in [7] by Minn, $[+B+B-B+B]$ proposed in [8]). Methods such as Minn's method work well in the AWGN channel, but the mean value might exhibit shifts from the exact start point due to the multipath effect. Alternatively a constant envelop preamble weighted by a PN sequence that gives an impulse shaped timing metric in AWGN channel similar to that obtained in [9] has been proposed in [10]. But, [9] and [10] methods have three drawbacks. The major disadvantage is that the metric's peak value is quite small compared to SC's metric in ISI or fading channels since it only considers one path correlation and other paths are treated as noise. It is hard to choose a threshold for detection and the probability of false alarm 
and missing detection is quite high. Another disadvantage is that in fading or ISI channels, the estimated start point will shift to the right and cause a large ISI when the instantaneous power of first path is not the biggest one among all paths. The third disadvantage is that since its metric cannot be calculated iteratively, the computation complexity is quite high when the number of the subcarriers is large.

Symbol timing estimation is often coupled with the CFO estimation. For example, [6] also provides a method to estimate the CFO, and [8] shows how Minn's training pattern (with repeated parts half the length of SC ones) [7] could be used to estimate the CFO. Since Minn's length of repeated parts of the training symbol is half of the SC's one, [8] shows Minn's training pattern yields little degradation compared to SC's training pattern.

A system which uses multiple antennas both on the transmitter and receiver is called a multiple-input multiple-output (MIMO) system. MIMO systems can be implemented in different ways to obtain either a spatial diversity gain to combat fading channel or a capacity gain in a multipath fading environment. When a MIMO system is designed for a frequency selective fading channel, to reduce the equalization complexity of the MIMO system, combining the MIMO system with OFDM modulation would be a good choice and the system becomes a MIMO-OFDM system [11].

MIMO-OFDM systems are also sensitive to synchronization errors. In [12], the author extended SC's synchronization method from OFDM systems to MIMO-OFDM systems. Due to the spatial diversity, the accuracy of the symbol timing estimator and CFO (fraction part) estimator is improved, but it did not give the method for estimating the integer part of the $\mathrm{CFO}$ and the estimated symbol timing shifted to the right. In [13] [14] and [15], the authors describe a synchronization method in the acquisition mode by four steps: coarse time synchronization and signal detection, frequency offset estimation in the time domain, residual frequency offset correction 
and fine time synchronization. This method uses several repeated parts with CP to do the synchronization and channel estimation. The preamble is quite efficient, but its residual frequency offset estimator is inaccurate in a frequency selective channel and no theoretic support for setting the threshold in fine time synchronization is given.

This thesis investigates synchronization methods for OFDM and MIMO-OFDM systems. Since synchronization method using the training symbols is more reliable than other methods, we focus on synchronization methods that use training symbols in this thesis. In particular, a novel synchronization technique is proposed by removing the $\mathrm{CP}$ and adding a flipped postfix (FIP) in the first training symbol structure to make the timing metric more steep and the timing estimator more accurate. Proposed synchronization methods for OFDM and MIMO-OFDM systems are in four steps: coarse time synchronization, estimation of the fraction part of the $\mathrm{CFO}$, estimation of the integer part of the $\mathrm{CFO}$ and fine time synchronization. Using analytical analysis, this thesis also explains how to set up the threshold for the fine time synchronization. Various FlP-based preambles are proposed and performance of the proposed synchronization technique is evaluated over AWGN, ISI and fading channels. For MIMO-OFDM systems, the performance of the proposed synchronization technique using different combining techniques and different number of antennas is evaluated under fading channels.

\subsection{Thesis contributions}

The main contribution of this thesis is the proposed postfix synchronization method for OFDM and MIMO-OFDM systems. By using FlP-based preambles in different channels, the symbol timing estimator becomes more accurate in term of the mean and variance of the estimator. A list of contributions is as follows: 
- Introducing FlP-based preambles (section 4.1.1 and section 4.2.1) to solve the uncertainty of the timing estimators in different channels. When the accuracy of the symbol timing estimator increases, the CFO estimator one also increases.

- Giving an analysis of the FlP-based preambles effect in an AWGN channel (section 4.1.3 and appendix A).

- Describing the fine time synchronization method for the proposed method and SC's method (section 4.1.2). It is shown in chapter 5 that the performance of the timing estimator much improves at low SNR by using the fine time synchronization.

- Analyzing the false alarm and missing detection probability for fine time estimator and giving a theoretical support for setting the threshold (appendix B).

- Extending the proposed method and SC's method from OFDM systems to MIMO-OFDM systems (section 4.2). Due to the spatial diversity, the performance of the symbol timing estimator and CFO estimator is much improved.

- Evaluating, comparing and discussing the performance of the proposed, SC's and Minn's synchronization methods under different channels (chapter 5).

\subsection{Thesis organization}

- Chapter 2, first, introduces the multipath fading channel and gives a tapped delay line model. Then it presents background on OFDM and MIMO-OFDM systems. Finally, it gives the system models used in this thesis.

- Chapter 3 gives a review of the previous works on OFDM and MIMO-OFDM 
systems synchronization and discusses the advantages and disadvantages of different synchronization methods.

- Chapter 4 describes the proposed methods for OFDM and MIMO-OFDM systems. Various FlP-based preambles are proposed for different channels and a theoretic analysis is given for AWGN channels.

- Chapter 5 gives the implementation details for the computer simulations. The proposed, Minn's and SC's methods are compared in terms of the mean and variance of the symbol timing estimator and CFO estimator in different channels by computer simulations. Different combining techniques and the effect of the antennas number are assessed for MIMO-OFDM systems.

- Chapter 6 summarizes the thesis and proposes future works. 


\section{Chapter 2}

\section{Background and system model}

\subsection{Multipath fading channel}

In a wireless communication environment, radio signals generally propagate according to three mechanisms: reflection, diffraction and scattering [16]. Due to these three mechanisms, plane waves received on the receiver antenna arrive from many different directions with random amplitudes, frequencies and phases. This phenomena is called multipath. Since the wavelength is relatively short, small changes in the location of the transmitter, receiver and scattering object in the environment will cause large changes in the phases of the incident plane wave components. The constructive and destructive addition of plane waves combined with motion results in envelope fading. This phenomenon is known as channel fading.

Multipath-fading results in a doubly dispersive channel that exhibits dispersion in both the time and frequency domains. Time dispersion arises because the multipath components propagate over transmission paths having different lengths and, hence, they reach the receiver antenna with different time delays. Time dispersion causes intersymbol interference (ISI) that can be mitigated by using a time or frequency 
domain equalizer in single carrier systems, a RAKE receiver in CDMA systems, or frequency domain equalization in OFDM systems. Channel time variations due to mobility are characterized by Doppler spreading in the frequency domain. Such time variant channels require an adaptive receiver to estimate and track the channel impulse response (CIR).

The complex baseband representation of a wireless channel impulse response can be described by [17]

$$
h(\tau ; t)=\sum_{n} \alpha_{n}(t) \delta\left(\tau-\tau_{n}(t)\right)
$$

where $\tau_{n}(t)$ is the $n^{\text {th }}$ path delay and $\alpha_{n}(t)$ is the corresponding complex amplitude. The complex baseband time variant channel impulse response $h(\tau ; t)$ is a complex valued Gaussian random process in the $t$ variable. When it is modeled as a zero mean complex valued Gaussian random process, which means none of the subpath is significantly stronger than others, the envelope $|h(\tau ; t)|$ at any instant $t$ is Rayleigh distributed and the channel is called a Rayleigh fading channel. When it is modeled as a nonzero mean complex valued Gaussian random process, which means there is one strong component along with the scatter components, the envelope $|h(\tau ; t)|$ at any instant $t$ is Ricean distributed and the channel is called a Ricean fading channel. There exist some other channel models, such as Nakagami, Weibull and Suzuki, derived mainly from experiments. This thesis focuses on the Rayleigh fading channel.

\subsubsection{Wide-Sense-Stationary with Uncorrelated Scattering (WSSUS) channel}

$h(\tau ; t)$ is commonly assumed to be wide-sense-stationary with uncorrelated scattering for the path delays. This channel is then referred to as a WSSUS channel. We define 
the autocorrelation function of $h(\tau ; t)$ as:

$$
E\left\{h^{*}\left(\tau_{1} ; t\right) h\left(\tau_{2} ; t+\Delta t\right)\right\}=\phi_{h}\left(\tau_{1} ; \Delta t\right) \delta\left(\tau_{1}-\tau_{2}\right)
$$

Let $\Delta t=0$, and we define

$$
\phi_{h}(\tau) \triangleq \phi_{h}(\tau ; 0)=E\left\{|h(\tau ; t)|^{2}\right\}
$$

$\phi_{h}(\tau)$ is called the multipath intensity profile or the delay power spectrum of the channel. $\phi_{h}(\tau)$ is the average power delay profile of the channel. The range of the value of $\tau$ over which $\phi_{h}(\tau)$ is essentially nonzero is called the multipath spread of the channel and is denoted as $T_{m}$. Let $\Phi_{H}(\Delta f)$ be the Fourier transform of $\phi_{h}(\tau)$. The range of the value of $\Delta f$ over which $\Phi_{H}(\Delta f)$ is essentially nonzero is called the coherence bandwidth of the channel and is denoted as $(\Delta f)_{H}$. The relationship of the multipath spread and the coherence bandwidth is $(\Delta f)_{H} \approx \frac{1}{T_{m}}$. Let $H(f ; t)$ be the Fourier transform of $h(\tau ; t)$ with respect to $\tau$. We define the autocorrelation function of $H(f ; t)$ to be $\Phi_{H}(\Delta f ; \Delta t)$, we have

$$
\Phi_{H}(\Delta f ; \Delta t)=E\left\{H(f+\Delta f ; t+\Delta t) H^{*}(f ; t)\right\}
$$

Let $\Delta f=0$, and we define $\Phi_{H}(\Delta t) \triangleq \Phi_{H}(0 ; \Delta t)$. The range of the value of $\Delta t$ over which $\Phi_{H}(\Delta t)$ is essentially nonzero is called the coherence time of the channel and is denoted as $(\Delta t)_{H}$. Let $S_{H}(\lambda)$ be the Fourier transform of $\Phi_{H}(\Delta t)$. The function $S_{H}(\lambda)$ is the power spectrum that gives the signal intensity as a function of the Doppler frequency $\lambda$ and it is called the Doppler power spectrum of the channel. The range of the value of $\lambda$ over which $S_{H}(\lambda)$ is essentially nonzero is called the Doppler spread of the channel and is denoted as $B_{d}$. The relationship of the Doppler spread and the 
coherence time is $(\Delta t)_{H} \approx \frac{1}{B_{d}}$.

The scattering function $S_{H}(\tau ; \lambda)$ which provides a measure of the power dispersion imposed by the channel over delay $\tau$ and Doppler frequency $\lambda$ is related to (2.4)

$$
S_{H}(\tau ; \lambda)=\int_{-\infty}^{\infty} \int_{-\infty}^{\infty} \Phi_{H}(\Delta f ; \Delta t) e^{-j 2 \pi \lambda \Delta t} e^{j 2 \pi \tau \Delta f} d \Delta f d \Delta t
$$

\section{Commonly-used Doppler power spectrum: Jakes/Clarke's model}

Jakes' model is a widely used model for the Doppler power spread spectrum of a mobile radio channel. In this model, $\Phi_{H}(\Delta t)=J_{0}\left(2 \pi f_{m} \Delta t\right)$ where $J_{0}(x)$ is the zero-order Bessel function of the first kind and $f_{m}=f_{c} v / c$ is the maximum Doppler frequency, where $f_{c}$ is the carrier frequency, $v$ is the vehicle speed in meters per second $(\mathrm{m} / \mathrm{s})$ and $c$ is the speed of light $\left(3 \times 10^{8} \mathrm{~m} / \mathrm{s}\right)$. The Doppler power spectrum is:

$$
S_{H}(\lambda)=\int_{-\infty}^{\infty} \Phi_{H}(\Delta t) e^{-j 2 \pi \lambda \Delta t} d \Delta t=\left\{\begin{array}{rc}
\frac{1}{\pi f_{m} \sqrt{1-\left(\lambda / f_{m}\right)^{2}}}, & \left(|\lambda| \leq f_{m}\right) \\
0, & \left(|\lambda|>f_{m}\right)
\end{array}\right.
$$

Commonly-used multipath intensity profile/power delay profile: exponential model

Exponential model is a widely used model for the power delay profile of a mobile radio channel. In this model,

$$
\phi_{h}(\tau)= \begin{cases}\frac{1}{\tau_{r m s}} e^{-\frac{\tau}{\tau_{r m s}}}, & \tau \geq 0 \\ 0, & \tau<0\end{cases}
$$

where $\tau_{r m s}$ is the standard deviation (or root-mean-square value) of the delay and is also equal to the mean of the delay in this model. The frequency correlation function 
is

$$
\Phi_{H}(\Delta f)=\frac{1}{2 \pi \Delta f \tau_{r m s}}
$$

In practice, a truncated version of (2.7) to a finite range is used.

Multipath delay spread leads to time dispersion and frequency selective fading [18]. Doppler spread leads to frequency dispersion and time selective fading. They are independent with each other.

\subsubsection{Fast fading vs. slow fading}

The channel can be classified either as a fast fading or slow fading channel when we compare the rate of transmitted baseband signal change with the rate of the channel change [18]. If the channel impulse response changes rapidly within the symbol duration, the channel is called a fast fading channel. That is, the coherence time of the channel is smaller than the symbol duration or the Doppler spread is greater than the bandwidth of the baseband signal. On the contrary, if the channel impulse response changes at a rate much slower than the transmitted baseband signal, the channel is called a slow fading channel. That is, the coherence time of the channel is much greater than the symbol duration or the Doppler spread is much less than the bandwidth of the baseband signal.

\subsubsection{Flat fading vs. frequency selective fading}

Time dispersion due to multipath makes the transmitted signal under either flat or frequency selective fading scenarios [18]. When a mobile radio channel has a constant gain and linear phase response over a bandwidth which is greater than the transmitted signal bandwidth, the channel is called a flat fading channel. The coherence bandwidth is much greater than the signal bandwidth $\left(W \ll(\Delta f)_{H}\right)$. On the contrary, 
when a channel has a constant gain and linear phase response over a bandwidth which is smaller than the bandwidth of transmitted signal, the channel is called a frequency selective fading channel. The spectrum of the transmitted signal has a bandwidth which is greater than the coherence bandwidth of the channel $\left(W>(\Delta f)_{H}\right)$. A frequency selective channel induces intersymbol interference (ISI). In this thesis, the bandwidth occupied by OFDM signals is greater than the coherence bandwidth of the channel. It undergoes frequency selective fading, but the bandwidth of each subcarrier is much smaller than the coherence bandwidth of the channel. Each subcarrier undergoes flat fading.

\subsubsection{A Tapped delay line channel model [17]}

A frequency selective slow fading channel can be represented by a tapped delay line channel model. Let $W$ be the bandwidth occupied by the real bandpass signal. Then the bandwidth of the equivalent lowpass signal $\tilde{s}(t)$ is $|f| \leq \frac{1}{2} W$. From the sampling theorem, we have

$$
\tilde{s}(t)=\sum_{n=-\infty}^{\infty} \tilde{s}\left(\frac{n}{W}\right) \frac{\sin [\pi W(t-n / W)]}{\pi W(t-n / W)}
$$

The Fourier transform of $\tilde{s}(t)$ is

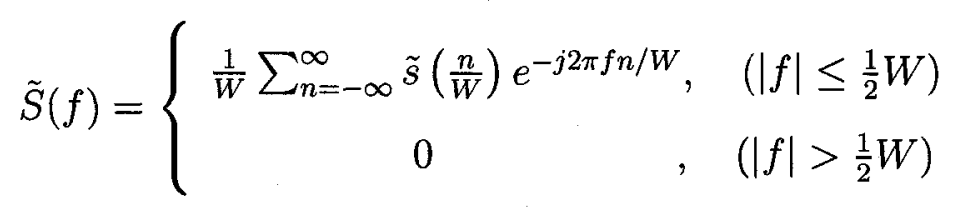

The noiseless received signal under a frequency selective channel is

$$
\begin{gathered}
\tilde{r}(t)=\int_{-\infty}^{\infty} H(f ; t) \tilde{S}(f) e^{j 2 \pi f t} d f \\
\left.\tilde{r}(t)=\int_{-\infty}^{\infty} H(f ; t) \tilde{S}(f) e^{j 2 \pi f t} d f \quad\right) d f \\
=\frac{1}{W} \sum_{n=-\infty}^{\infty} \tilde{s}\left(\frac{n}{W}\right) \int_{-\infty}^{\infty} H(f ; t) e^{j 2 \pi f\left(t-\frac{n}{W}\right)} d f
\end{gathered}
$$




$$
\begin{aligned}
& =\frac{1}{W} \sum_{n=-\infty}^{\infty} \tilde{s}\left(\frac{n}{W}\right) h\left(t-\frac{n}{W} ; t\right) \\
& =\frac{1}{W} \sum_{n=-\infty}^{\infty} \tilde{s}\left(t-\frac{n}{W}\right) h\left(\frac{n}{W} ; t\right)
\end{aligned}
$$

where $h(\tau ; t)$ is the time variant channel impulse response (CIR) and $H(f ; t)$ is the transfer function. We define

$$
h_{n}(t)=\frac{1}{W} h\left(\frac{n}{W} ; t\right)
$$

Then we have

$$
\tilde{r}(t)=\sum_{n=-\infty}^{\infty} h_{n}(t) \tilde{s}\left(t-\frac{n}{W}\right)
$$

From (2.13), the time variant frequency selective channel can be modeled as a tapped delay line with tap space $\frac{1}{W}$ and tap weight coefficients $\left\{h_{n}(t)\right\}$. The lowpass channel impulse response is

$$
h(\tau ; t)=\sum_{n=-\infty}^{\infty} h_{n}(t) \delta\left(\tau-\frac{n}{W}\right)
$$

It achieves a resolution of $\frac{1}{W}$ in the multipath delay profile. In practical scenarios, since the total multipath spread is $T_{m}$, we truncate the tapped delay line at $M=\left\lfloor T_{m} W\right\rfloor+1$ taps and we have

$$
\tilde{r}(t)=\sum_{n=1}^{M} h_{n}(t) \tilde{s}\left(t-\frac{n}{W}\right)
$$

In a Rayleigh fading channel, the tap weights $\left\{h_{n}(t)\right\}$ are zero mean complex Gaussian random processes and they are statistically independent. The power delay profile of the channel, (2.3), determines each tap's mean power, or equivalently the variance of the $h_{n}(t)$. We assume all taps have the same Doppler power spectrum and 
we can use the Jakes' model in section 2.1.1 to generate the tap weights.

Alternatively, for each tap $h_{n}(t)$, if the amplitude of $h_{n}(t)$ is fixed and the phase of $h_{n}(t)$ is fixed or uniformly distributed in $[0,2 \pi$ ) (Phase does not change in the $t$ variable), then (2.15) can be used to model ISI channels.

\subsection{Representation of OFDM signal}

OFDM is a multicarrier modulation technique which transmits data in parallel by modulating a set of orthogonal subcarriers. OFDM is attractive because it is achieving a high data rate under a frequency selective fading channel, is easy to equalize in the frequency domain (only a one-tap frequency domain equalizer is required) and allows the water pouring strategy (using adaptive modulation) to increase the overall bandwidth efficiency.

\subsubsection{Transmitted OFDM signal (the simplest version)}

OFDM is a modulation technique that spreads the data over a large number of carriers which are spaced apart at frequencies $f_{n}=n W / N$, where $n$ is an integer, $W$ is the total available bandwidth and $N$ is the total number of subcarriers [3]. In the most simple case $W=N / T_{s}$, where $T_{s}$ is the symbol duration. With this choice of subcarriers, the subcarriers are mutually orthogonal, since

$$
\int_{k T_{s}}^{(k+1) T_{s}} e^{j 2 \pi f_{i} t} \cdot e^{-j 2 \pi f_{l} t} d t=T_{s} \delta_{i l}
$$

where $\delta_{i l}$ is Kronecker delta: $\forall i \neq l \delta_{i l}=0$ and $\delta_{l l}=1$.

The modulation at this stage normally uses QPSK, DQPSK, QAM or any other modulation scheme. Let the complex transmit symbol at time instant $k$ on the $i^{\text {th }}$ subcarrier be $X_{i, k}$. Consider QPSK, $X_{i, k}=a_{i, k}+j b_{i, k}$, with $a_{i, k}, b_{i, k}= \pm 1$, with 
$-N / 2 \leq i \leq N / 2$ and $i \neq 0$ (DC-subcarrier can be distorted by the electronics and therefore is usually not used). For simplicity of notation in this thesis, we use $0 \leq i \leq N-1$ instead of $-N / 2 \leq i \leq N / 2$ and $i \neq 0$.

The OFDM signal is

$$
s(t)=\Re\left\{\tilde{s}(t) e^{j 2 \pi f_{c} t}\right\}
$$

where $f_{c}$ is the center frequency of the occupied frequency spectrum and $\tilde{s}(t)$ is the complex envelope of the OFDM signal given by

$$
\tilde{s}(t)=\sum_{k=-\infty}^{\infty} \tilde{s}_{k}(t)=\sum_{k=-\infty}^{\infty} \sum_{i=0}^{N-1} X_{i, k} g_{i}\left(t-k T_{s}\right)
$$

where the basis pulse $g_{i}(t)$ is a frequency-shifted rectangular pulse

$$
g_{i}(t)= \begin{cases}e^{j 2 \pi f_{i} t}=e^{j 2 \pi i t / T_{s}} & 0 \leq t \leq T_{s} \\ 0 & \text { otherwise }\end{cases}
$$

\subsubsection{OFDM digital implementation}

We can use $N$ oscillators to generate $N$ subcarriers, then use $N$ filters in the receiver to get the data. When $N$ is large, the bandwidth of the subcarrier might be very narrow. It is hard and expensive to build $N$ precise oscillators and filters. In 1971, Weinstein, S. and Ebert, P. published the paper [19] which introduces using the Discrete Fourier Transform (DFT) to implement the frequency division multiplexing (FDM). The DFT transforms a cyclic time domain signal into its equivalent frequency spectrum. This is done by finding the equivalent waveform generated by a sum of orthogonal sinusoidal components. The Inverse Discrete Fourier Transform (IDFT) performs the reverse process, transforming a spectrum signal (amplitude and phase of each component) 
into a time domain signal. IDFT operation can be used to generate OFDM signals as shown in the following.

Consider only the first symbol interval and assume perfect synchronization in time and frequency domain. From (2.18), we have

$$
\tilde{s}_{0}(t)=\sum_{i=0}^{N-1} X_{i, 0} g_{i}(t)
$$

If we sample $\tilde{s}_{0}(t)$ in $(2.20)$ at $t=\frac{n \times T_{s}}{N}, n=0,1, \cdots, N-1$, we have

$$
\tilde{s}_{0}\left(\frac{n \times T_{s}}{N}\right)=\sum_{i=0}^{N-1} X_{i, 0} e^{\frac{j 2 \pi i n}{N}}, n=0,1, \cdots, N-1
$$

since $\frac{T_{s}}{N}$ is a constant, we can write $\tilde{s}_{0}\left(\frac{n \times T_{s}}{N}\right)$ to be

$$
\tilde{s}_{0}(n)=\sum_{i=0}^{N-1} X_{i, 0} e^{\frac{j 2 \pi i n}{N}}, n=0,1, \cdots, N-1
$$

The IDFT of the $X_{i, 0}, i=0,1, \cdots, N-1$ is

$$
s(n)=\frac{1}{N} \sum_{i=0}^{N-1} X_{i, 0} e^{\frac{j 2 \pi i n}{N}}, n=0,1, \cdots, N-1
$$

Obviously, (2.22) and (2.23) are identical except for a constant scalar $\frac{1}{N}$. Hence it is seen that the OFDM signal can be generated at baseband by taking the IDFT of the complex modulated data symbols instead of generating the carriers separately. For the same reason, the receiver can be also implemented using the DFT.

The FFT or IFFT provides an efficient way to implement the DFT or IDFT [20]. It reduces the number of complex multiplications from $N^{2}$ to $\frac{N}{2} \log _{2} N$ for N-point DFT or IDFT. Hence, with the help of the FFT and IFFT, the implementation of 
OFDM is very simple.

\subsubsection{Adding Cyclic Prefix}

After OFDM modulation, a guard interval is inserted to suppress ISI which is caused by the multipath fading propagation. The guard interval is also called the cyclic prefix. As shown in Fig. 2.1, it is a copy of the last part of the present symbol. We

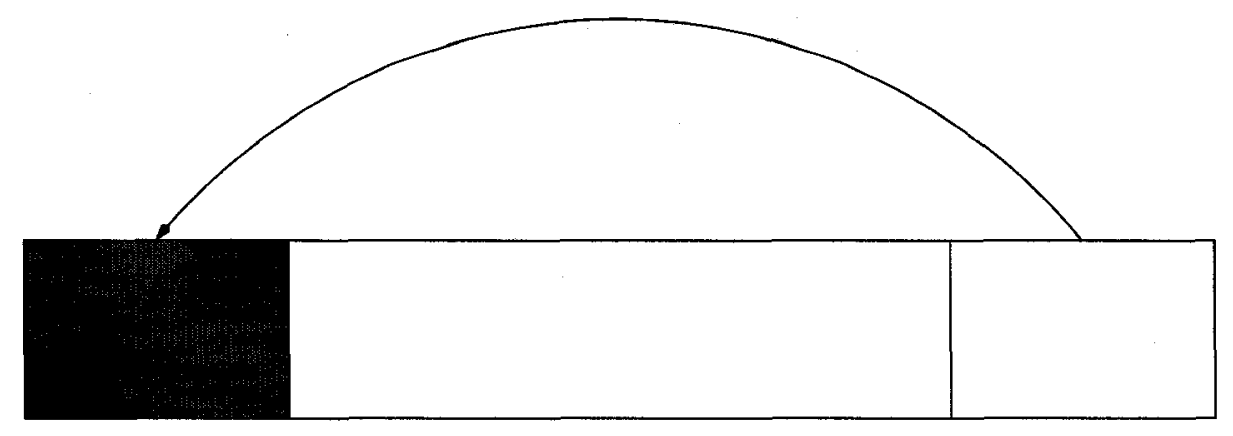

Time

Fig. 2.1 The cyclic prefix is a copy of the last part of the present symbol

define a new base function

$$
g_{i}^{\prime}(t)=e^{j 2 \pi i \frac{W_{s}}{N} t} \quad-T_{\mathrm{cp}} \leq t \leq T_{F F T}
$$

where $W_{s}$ is the signal bandwidth excluding the bandwidth occupied by the windowing and CP. $T_{F F T}=\frac{N}{W_{s}}$ is the duration of the OFDM symbol excluding the windowing and $\mathrm{CP}$ and $T_{\mathrm{cp}}$ is the duration of the cyclic prefix. Hence the symbol duration is $T_{s}=T_{F F T}+T_{\mathrm{cp}}$ in this stage. It can be verified that

$$
g_{i}^{\prime}\left(t+N / W_{s}\right)=e^{j 2 \pi i \frac{W_{s}}{N}\left(t+N / W_{s}\right)}=e^{j 2 \pi i \frac{W_{s}}{N} t}=g_{i}^{\prime}(t)
$$


Considering the first symbol interval, if we use $g_{i}^{\prime}(t)$ instead of $g_{i}(t)$ in $(2.20)$, the complex envelope of the first OFDM signal is

$$
\tilde{s}_{0}(t)=\sum_{i=0}^{N-1} X_{i, 0} g_{i}^{\prime}(t) \quad-T_{\mathrm{cp}} \leq t \leq T_{F F T}
$$

Therefore, for $0 \leq t \leq T_{F F T}, \tilde{s}_{0}(t)$ is equal to the "normal" OFDM signal (given for example by (2.20)). For $-T_{\mathrm{cp}} \leq t<0$, we obtain

$$
\tilde{s}_{0}(t)=\sum_{i=0}^{N-1} X_{i, 0} g_{i}^{\prime}(t)=\sum_{i=0}^{N-1} X_{i, 0} g_{i}^{\prime}\left(t+N / W_{s}\right)=\tilde{s}_{0}\left(t+N / W_{s}\right)
$$

Hence $\tilde{s}_{0}(t)$ is a copy of the last part of the present symbol. Equivalently, the samples of the complex envelope of the OFDM signal (with CP) are:

$$
\tilde{s}_{n}=\tilde{s}_{0}\left(n T_{F F T} / N\right)= \begin{cases}\sum_{i=0}^{N-1} X_{i, 0} e^{j 2 \pi i n / N} & n=0, \ldots, N-1 \\ \tilde{s}_{0}\left((n+N) \frac{N}{W_{s}} / N\right)=\tilde{s}_{N+n} & n=-G, \ldots,-1\end{cases}
$$

where $G$ is the number of the cyclic prefix samples. Similarly, if several data symbols are considered, then the complex envelope of the OFDM signal (with $\mathrm{CP}$ ) is given by $^{1}$

$$
\tilde{s}(t)=\sum_{k=-\infty}^{\infty} \sum_{i=0}^{N-1} X_{i, k} g_{i}^{\prime}\left(t-k T_{s}\right)
$$

where $g_{i}^{\prime}(t)$ is given by $(2.24)$ and $T_{s}=T_{F F T}+T_{\mathrm{cp}}=N / W_{s}+G / W_{s}$.

When the duration of cyclic prefix is longer than the delay spread of the channel, the ISI caused by the multipath fading propagation would be diminished. All the delay echoes of the previous symbol will die away in the cyclic prefix. Otherwise the ISI will degrade the performance. The cyclic prefix makes OFDM robust against ISI

\footnotetext{
${ }^{1}$ Note that in (2.29), a rectangular pulse shape is assumed.
} 
and ICI.

\subsubsection{Adding windowing}

The windowing would be applied after adding the cyclic prefix to reduce out-of-band frequency leaking when the OFDM symbols have a discontinuous phase connection at the begin and end. For example, the windowing function $w(t)$ is the transmitter pulse prototype to smooth each OFDM symbol with a window around the edges of the OFDM frame to reduce out of band radiation resulting from Sinc function sidelobes. It is defined by:

$$
w(t)=\left\{\begin{array}{cl}
\frac{1}{2}\left[1+\cos \frac{\pi\left(t+T_{c p}\right)}{T_{w i n}}\right] & -T_{w i n}-T_{c p} \leq t \leq-T_{c p} \\
1 & -T_{c p} \leq t \leq T_{F F T} \\
\frac{1}{2}\left[1+\cos \frac{\pi\left(t-T_{F F T}\right)}{T_{w i n}}\right] & T_{F F T} \leq t \leq T_{F F T}+T_{w i n}
\end{array}\right.
$$

where $T_{w i n}$ is the duration of the windowing (one side).

\subsubsection{Transmitted OFDM signal (including CP and windowing)}

The complex envelope of the OFDM signal (with CP and windowing) is given by

$$
\tilde{s}(t)=\sum_{k=-\infty}^{\infty} \sum_{i=0}^{N-1} X_{i, k} e^{\frac{j 2 \pi i W_{s}\left(t-k T_{s}\right)}{N}} w\left(t-k T_{s}\right)
$$

where $T_{s}=2 T_{w i n}+T_{c p}+T_{F F T}$.

\subsubsection{Received signal (assuming perfect synchronization)}

The complex envelope of the OFDM signal can be represented by (2.31). Assume that the transmitted OFDM signal goes through a time variant multipath fading channel which is expressed by its equivalent lowpass impulse response $h(\tau ; t)$ plus additive 
white Gaussian noise (AWGN) $n(t)$, then the lowpass equivalent form of the received signal can be written as

$$
\tilde{r}(t)=h(\tau ; t) \circledast \tilde{s}(t)+n(t)=\int_{0}^{\tau_{\max }} h(\tau ; t) \tilde{s}(t-\tau) d \tau+n(t)
$$

where $\circledast$ is the convolution operator and $\tau_{\max }$ is the channel maximum delay (or delay spread). $h(\tau ; t)$ denotes the impulse response at time $t$ ( $\tau$ denotes the excess delay of the channel impulse response), which is zero outside the range of this convolution integral $\tau=\left[0, \tau_{\max }\right]$ and $\tilde{s}(t)$ is the complex envelope of the OFDM signal given by $(2.31)$.

Assuming the channel is time-invariant during the transmission of one OFDM symbol (slow fading channel), $\tau_{\max }<T_{c p}$, and there is perfect time synchronization, the orthogonality of the tones is preserved and there is no interference from the previous OFDM symbol to affect the current one. In other words, ISI is suppressed. We assume that $h(\tau ; t)$ is time invariant in one OFDM symbol interval and it can be represented as $h(\tau)$. In that case, considering the first symbol interval, the received signal after removing the windowing and $\mathrm{CP}$ can be represented by:

where

$$
\begin{gathered}
\tilde{r}_{0}(t)=h(\tau ; t) \circledast \tilde{s}_{0}(t)+n(t)=\int_{0}^{\tau_{\max }} h(\tau) \tilde{s}_{0}(t-\tau) d \tau+n(t) \\
\tilde{r}_{0}(t)=h(\tau ; t) \circledast \tilde{s}_{0}(t)+n(t)=\int_{0}^{\tau_{\max }} h(\tau) \tilde{s}_{0}(t-\tau) d \tau+n(t) \\
\tilde{s}_{0}(t)=\sum_{i=0}^{N-1} X_{i} e^{\frac{j 2 \pi i t}{T_{F F T}}}
\end{gathered}
$$

and $X_{i}$ is $X_{i, 0}$. Sampling the received signal at $n T_{F F T} / N$ yields

$$
\tilde{r}_{0}\left(n T_{F F T} / N\right)=\int_{0}^{\tau_{\max }} h(\tau) \tilde{s}_{0}\left(n T_{F F T} / N-\tau\right) d t+\tilde{n}_{n}
$$


assuming a tapped delay line channel model (section 2.1.4)

$$
h(t)=\sum_{m=0}^{M-1} h_{m} \delta\left(t-m T_{F F T} / N\right)
$$

we have

$$
\begin{aligned}
\tilde{r}_{0}\left(n T_{F F T} / N\right) & =\int_{0}^{\tau_{\max }} \sum_{m=0}^{M-1} h_{m} \delta\left(\tau-m T_{F F T} / N\right) \tilde{s}_{0}\left(n T_{F F T} / N-\tau\right) d t+\tilde{n}_{n} \\
& =\sum_{m=0}^{M-1} h_{m} \tilde{s}_{0}\left((n-m) T_{F F T} / N\right)+\tilde{n}_{n} \\
& =\sum_{m=0}^{M-1} h_{m} \tilde{s}_{0}(n-m)+\tilde{n}_{n}
\end{aligned}
$$

where $\tilde{s}_{0}(n-m)$ is a short notation for $\tilde{s}_{0}\left((n-m) T_{F F T} / N\right)$. An AWGN channel corresponds to $h_{0}=1$ and $h_{1}=h_{2} \cdots=h_{m-1}=0$ (i.e. $h(\tau)=\delta(\tau)$ ).

Assuming an AWGN channel and no noise, the received samples are given by

$$
\tilde{r}_{0}\left(n T_{F F T} / N\right)=\tilde{s}_{0}(n)=\left.N \cdot \operatorname{IFFT}\left\{X_{i, 0}\right\}\right|_{n^{t h} \text { sample }}
$$

Therefore, it is seen that $\left\{X_{i, 0}\right\}$ can be attained from $\left\{\tilde{r}_{0}\left(n T_{F F T} / N\right)\right\}$ by performing an FFT.

An FFT operation can also be used to attain the $X_{i, 0}$ 's for a tapped delay line channel model as shown as follows. In that case, the output of the FFT is

$$
\begin{aligned}
Y_{k} & =\left.F F T\left\{\tilde{r}_{0}\left(i T_{F F T} / N\right)\right\}\right|_{k^{t h} \text { sample }}=\sum_{i=0}^{N-1} \tilde{r}_{0}\left(i T_{F F T} / N\right) e^{-\frac{j 2 \pi i k}{N}} \\
& =\sum_{i=0}^{N-1}\left\{\sum_{m=0}^{M-1} h_{m} \tilde{s}_{0}(i-m)+\tilde{n}_{i}\right\} e^{-\frac{j 2 \pi i k}{N}}
\end{aligned}
$$




$$
\begin{aligned}
& =\sum_{m=0}^{M-1} h_{m} e^{-\frac{j 2 \pi m k}{N}}\left\{\sum_{i=0}^{N-1} \tilde{s}_{0}(i-m) e^{-\frac{j 2 \pi(i-m) k}{N}}\right\}+\sum_{i=0}^{N-1} \tilde{n}_{i} e^{-\frac{j 2 \pi i k}{N}} \\
& =\sum_{m=0}^{M-1} h_{m} e^{-\frac{j 2 \pi m k}{N}}\left\{\sum_{i=0}^{N-1}\left[\sum_{l=0}^{N-1} X_{l, 0} e^{\frac{j 2 \pi(i-m) l}{N}}\right] e^{-\frac{j 2 \pi(i-m) k}{N}}\right\}+N_{k} \\
& =H_{k}\left\{\sum_{l=0}^{N-1} X_{l, 0} e^{\frac{j 2 \pi(k-l) m}{N}} \sum_{i=0}^{N-1} e^{\frac{j 2 \pi(l-k) i}{N}}\right\}+N_{k}
\end{aligned}
$$

where $N_{k}=\sum_{i=0}^{N-1} \tilde{n}_{i} e^{-\frac{j 2 \pi i k}{N}}$ is the independent noise sample and $H_{k}=\sum_{m=0}^{M-1} h_{m} e^{-\frac{j 2 \pi m k}{N}}$. Since $\sum_{i=0}^{N-1} e^{\frac{j 2 \pi(l-k) i}{N}}=\delta_{k l}$, we have

$$
\begin{aligned}
Y_{k} & =H_{k}\left\{\sum_{l=0}^{N-1} X_{l, 0} e^{\frac{j 2 \pi(k-l) m}{N}} \sum_{i=0}^{N-1} e^{\frac{j 2 \pi(l-k) i}{N}}\right\}+N_{k} \\
& =H_{k}\left\{\sum_{l=0}^{N-1} X_{l, 0} e^{\frac{j 2 \pi(k-l) m}{N}} \delta_{k l}\right\}+N_{k} \\
& =H_{k} X_{k, 0}+N_{k}
\end{aligned}
$$

therefore the complex data symbols can be attained from the $Y_{k}$ 's using an one-tap frequency domain equalizer.

Remark, note that (2.40) can be viewed as the output of a matched filter, matched to the $k^{\text {th }}$ subcarrier signal, assuming no ICI i.e.

$$
Y_{k}=\frac{1}{T_{F F T}} \int_{0}^{T_{F F T}} \tilde{r}_{0}(t) \cdot e^{-j \frac{2 \pi k t}{T_{F F T}}} d t
$$

\subsubsection{The OFDM system block diagram}

The OFDM system block diagram is shown in Fig. 2.2 [3]. At the transmitter, the input binary data stream is encoded using any suitable modulation technique. Then this serial data stream is converted to the parallel data stream. This parallel data stream is then taken through an IFFT and this stage is called the OFDM modulation. 
After OFDM modulation, a cyclic prefix is inserted to suppress ISI which is caused by the multipath fading propagation. The windowing would be applied after the cyclic prefix to reduce out-of-band frequency leaking. After that, the signal though a D/A converter becomes an analog baseband signal, upconverted to RF, and transmitted. After passing a time variant channel and adding a Gaussian noise, the signal arrives at the receiver antennas. For an OFDM receiver, the signal downconverted into an analog baseband signal, is passed through an A/D converter, then windowing and CP are removed, after the signal is demodulated using FFT (that converts the OFDM time domain samples into the frequency domain samples which correspond to the complex modulated data symbols). Then the obtained parallel data sequences are converted into a serial data sequence. After decoding, it becomes the output binary data stream.

Throughout this thesis, we employ the following assumptions:

- A cyclic prefix is used.

- The impulse response of the channel is shorter than the cyclic prefix.

- Channel noise is additive, white, and complex Gaussian.

- The fading is slow enough for the channel to be considered constant during one symbol interval. (For time variant fading channels, we model the CIR changes every sample.) 


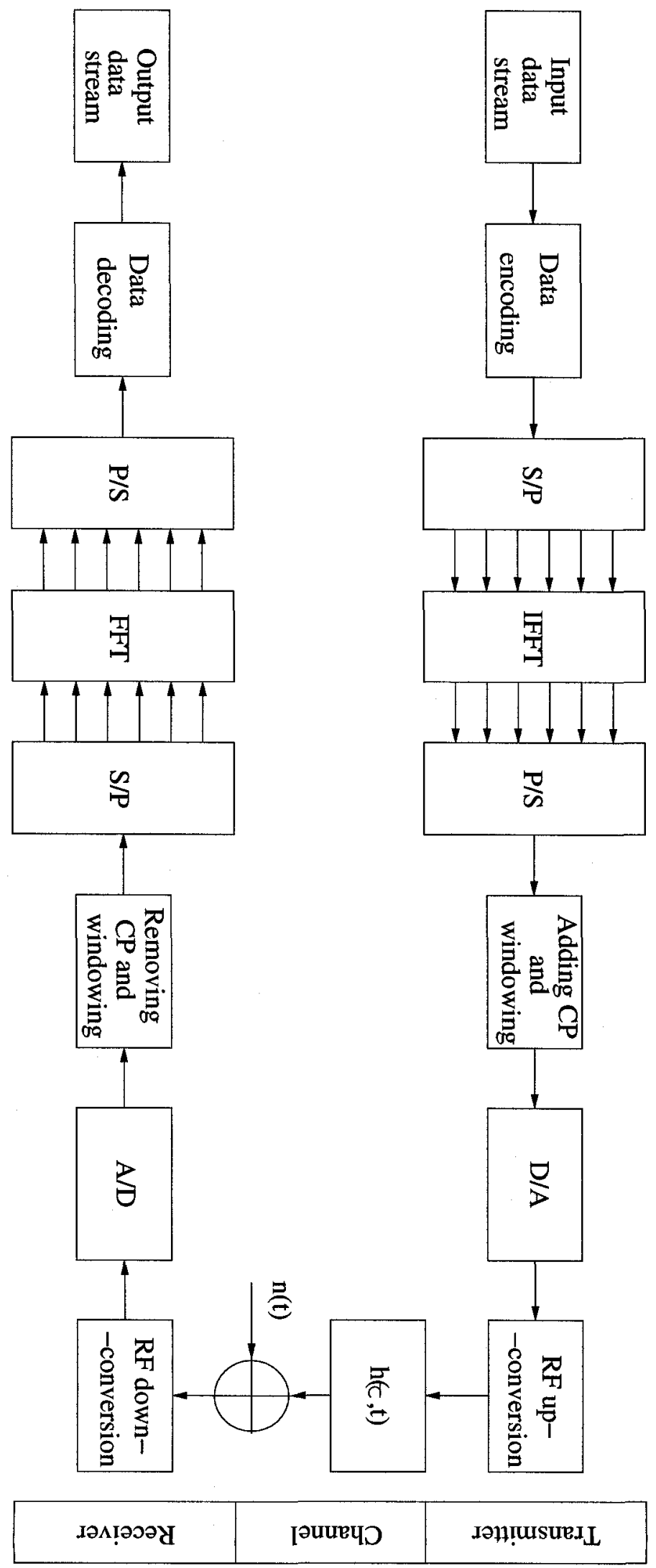

Fig. 2.2 The OFDM system block diagram 


\subsection{OFDM issues}

\subsubsection{Coding and Modulation}

In the OFDM system the data is transmitted by $N$ narrow subcarriers [1]. Each subcarrier has a different amplitude when it arrives to the receiver. Some of them may be in the deep fading in a frequency selective fading channel. That would cause high BER although most of subcarriers are detected without errors. To avoid performance dominated by the weakest subcarriers, some redundant codes are used to make the performance determined by the average received power. In the OFDM system block codes and/or convolution codes are normally used, sometimes two codes are combined or concatenated.

To deal with error bursts, we can use interleaving to randomize the occurrence of bit errors prior to decoding. After interleaving the adjacent bits are separated by several bits. Coding and interleaving can be combined to deal with the PAPR.

Because the rectangular constellations of QAM are easy to implement as they can be split into independent PAM components for both the in-phase and the quadrature part, we normally use them in combination with OFDM. The adaptive modulation uses different modulation schemes on different subcarriers according to the channel information of each subcarrier. The water pouring strategy increases the overall bandwidth efficiency.

\subsubsection{Issues related to Cyclic Prefix}

The cyclic prefix makes OFDM robust against the ISI and ICI. Some algorithms use it for the synchronization, but it takes $5 \%$ to $30 \%$ of the symbol duration. That makes it inefficient in terms of spectrum use. Another disadvantage is the SNR loss. The cyclic prefix constitutes "noise" when the signal is concerned and provides less power to the 
signal frame by occupying signal space. By using a proper equalizer and new detection algorithm [21] we can eliminate the cyclic prefix. In [22], the author suggests to use a binary sequence that is multiplexed with the OFDM signal to replace the cyclic prefix. The sequence is used as a pilot for the time and frequency domain synchronization but we have to use a good equalizer reduce the ISI. The advantage is that we can put some information in that binary sequence, such as the user ID. In [22] the author says this method can also reduce the PAPR. It also increases the spectrum efficiency. We can also use those PN binary sequence to estimate the channel. The disadvantage is that we have to use a complex equalizer. In this thesis, we only consider a CP-based OFDM system.

\subsubsection{The Peak to Average Power Ratio problem}

Because of the coherent summation of the sine waves, we have a large peak to average power ratio which makes the problem for the linear bandwidth of the RF amplifier [23]. When $N$ signals are added with the same phase, they produce a peak power that is $N$ times the average power. To reduce the PAPR, we can use techniques such as amplitude clipping, clipping and filtering, coding, peak windowing tone reservation, tone injection, active constellation extension, partial transmit sequence, selected mapping and interleaving, but the price is the performance degradation and complexity increase.

\subsubsection{Synchronization of OFDM systems [3]}

Synchronization is an important issue of OFDM systems since it makes each subcarrier orthogonal with others. OFDM systems are highly sensitive to timing errors, carrier frequency offsets, phase noise and sampling clock offsets. The symbol synchronization means knowing when the symbol starts to make the symbol time offset $\epsilon$ as small as 
possible. If it is larger than the cyclic prefix, the orthogonality is not maintained and ICI will occur. Carrier frequency offset is created by differences of the oscillators in transmitter and receiver, Doppler shifts or phase noise are introduced by nonlinear channels. Two destructive effects are caused by a carrier frequency offset in OFDM systems. CFO reduces the signal amplitude and introduces ICI from other subcarriers. Normally, the synchronization algorithms rely on the evaluation of the correlation function (training symbol or cyclic prefix).

\section{Symbol time and carrier frequency offset}

Symbol time and carrier frequency offset cause ICI. For single carrier systems the phase noise and frequency offset only cause the degradation of SNR rather than interference. OFDM is more sensitive to the frequency offset.

Assuming perfect synchronization, the received signal after the demodulation of OFDM (using FFT) becomes the received signal constellation point $Y_{k}$ given by $(2.40)$. Due to a frequency mismatch between the oscillators of the transmitter and receiver, a frequency offset $\delta_{f}$ and a carrier phase offset $\theta$ must be accounted for as a frequency shift in the lowpass equivalent received signal:

$$
\tilde{r}^{\prime}(t)=\tilde{r}_{0}(t) \cdot e^{j\left(2 \pi \delta_{f} t+\theta\right)}
$$

where $\tilde{r}_{0}(t)$ is given by (2.33). If furthermore a symbol time offset $\epsilon$ is present, the $k^{\text {th }}$ sample of the received signal is

$$
r_{k}=\tilde{r}^{\prime}\left(k T_{F F T} / N+\epsilon\right)=\tilde{r}_{0}\left(k T_{F F T} / N+\epsilon\right) \cdot e^{j\left(2 \pi \delta_{f}\left(k T_{F F T} / N+\epsilon\right)+\theta\right)}
$$

Assume for simplicity, $\epsilon=-n_{\epsilon} \frac{T_{F F T}}{N}$, where $n_{\epsilon}$ is an integer representing the unknown 
arrival time of a symbol. Let $\gamma=\delta_{f} T_{F F T}$ be the CFO normalized by the subcarrier spacing. From (2.43) and (2.37), we have

$$
\begin{aligned}
r_{k} & =\tilde{r}_{0}\left(\left(k-n_{\epsilon}\right) \frac{T_{F F T}}{N}\right) \cdot e^{j\left(2 \pi\left(k \frac{\gamma}{N}-n_{\epsilon} \frac{\gamma}{N}\right)+\theta\right)} \\
& =\left\{\sum_{m=0}^{M-1} h_{m} \tilde{s}_{0}\left(k-n_{\epsilon}-m\right)+\tilde{n}_{k-n_{\epsilon}}\right\} e^{j\left(2 \pi \frac{k \gamma}{N}+\theta_{0}\right)} \\
& =\tilde{s}_{k-n_{\epsilon}} e^{j\left(2 \pi \frac{k \gamma}{N}+\theta_{0}\right)}+n_{k}
\end{aligned}
$$

where $\theta_{0}=\theta-2 \pi \frac{n_{\epsilon} \gamma}{N}$ is the initial phase. Note that demodulation can be done either using (2.40) or (2.41). In practice (2.40) is implemented. However to analyze qualitatively the effect of non-integer symbol time offset, it is more convenient to use (2.41). In the presence of a symbol time offset $\epsilon$, the demodulation interval becomes $t \in\left(\epsilon, T_{F F T}+\epsilon\right)$. Hence we have

$$
Y_{k}=\frac{1}{T_{F F T}} \int_{\epsilon}^{T_{F F T}+\epsilon} \tilde{r}^{\prime}(t) \cdot e^{-j 2 \pi k \frac{(t-\epsilon)}{T_{F F T}}} d t
$$

From [3], the final expression for the received signal without ICI term becomes:

$$
Y_{k}=X_{k} \cdot H_{k} \cdot \operatorname{sinc}\left(\delta_{f} \cdot T_{F F T}\right) \cdot e^{j \Psi_{k}}+N_{k}
$$

and

$$
\Psi_{k}=\theta+2 \pi \delta_{f}\left(\frac{T_{F F T}}{2}+\epsilon\right)+2 \pi \epsilon\left(\frac{k}{T_{F F T}}\right)
$$

where $H_{k}=H\left(\frac{k}{T_{F F T}}\right)=\int_{0}^{\tau_{\max }} h(\tau) \cdot e^{-j 2 \pi k\left(\frac{\tau}{T_{F F T}}\right)} d \tau$ is the Fourier transform of $h(\tau)$ at the frequency $\frac{k}{T_{F F T}}, N_{k}$ is the independent noise sample, and $\operatorname{sinc}(x)=\sin (\pi x) /(\pi x)$. From (2.46), we can find the carrier frequency offset $\delta_{f}$ and carrier phase offset $\theta$ will cause attenuation of all subcarriers by the Sinc function and a common phase 
rotation. $H_{k}$ will introduce a phase rotation and an attenuation too. To minimize the effect of channel, we can estimate the channel when we assume the timing offset is not very large, or use differential modulation to cancel out the effect of the channel in a consecutive sequence. The carrier frequency offset also causes ICI of the adjacent subcarriers. The symbol time offset $\epsilon$ causes a progressive phase rotation (multiply by $k$ ) and the maximum phase rotation is found at the edges of the frequency band. When $\tau_{\text {max }}-T_{c p} \leq \epsilon \leq 0$, that means the channel impulse response dies away in the guard interval and no ISI, otherwise we have to deal with the ISI by the equalizer.

\section{Sampling frequency synchronization}

The received continuous time signal is sampled at instants determined by the receiver clock. There are two methods to deal with the mismatch in the sampling frequency. In the synchronized sampling system, a timing algorithm controls a VCO (voltage controlled oscillator) to lock the receiver clock with the transmitter clock. In the non-synchronized sampling system, the sampling rate is fixed and a postprocessing is required in the digital domain. The effect of the clock frequency offset not only rotates and attenuates the useful signal component but also introduces ICI. Nonsynchronized sampling is more sensitive to the frequency offset, compared with a synchronized sampling system. In this thesis, we assume perfect sampling frequency synchronization and will focus on symbol time and carrier frequency offset estimation.

\subsection{MIMO-OFDM systems background}

\subsubsection{MIMO systems}

According to the number of antennas which are used at the transmitter and receiver, communication systems can be classified as four kinds [3][13]. Single-Input Single- 
Output (SISO) is the basic wireless configuration, Single-Input Multiple-Output (SIMO) uses a single receiver antenna and multiple transmitter antennas, Multiple-Input Single-Output (MISO) has multiple transmitter antennas and single receiver antenna, Multiple-Input Multiple-Output (MIMO) uses multiple antennas both for the transmitter and receiver. MIMO systems can be implemented in different ways to obtain either a spatial diversity gain to combat fading channel or a capacity gain.

Spatial diversity is also called antenna diversity which can be divided into receive diversity and transmit diversity. Such techniques include delay diversity, space time block codes (STBC) and space time trellis codes (STTC). Types of diversity schemes include selection diversity, maximum ratio diversity and equal gain diversity. Transmit diversity is easy to be implemented at the base station but the transmitter must know well the channel information or use the space-time coding schemes without knowledge of the channel.

Spatial multiplexing gives systems a capacity gain. One popular example is Vertical Bell Laboratories Layered Space Time (V-BLAST) system. A MIMO system can linearly increase the channel capacity by using the same bandwidth spectrum and boosts the data rate multiple times, when a rich scattering environment provides independent transmission paths from each transmitter antenna to each receiver antenna. Each transmitter antenna transmits different data at the same time. Because of the multipath propagation, each receiver antenna gets the mixed data. Since we assume that the receiver has the complete knowledge about the channel, the data are processed by the signal processor to reconstruct the transmitted signal. It looks like using $N$ different cables in same frequency band to transmit parallel data. It increases symbol rate $N$ times and also reuses the spectrum $N$ times.

The third way to use MIMO systems is to exploit the knowledge of the channel at the transmitter. It decomposes the channel coefficient matrix by singular value 
decomposition (SVD), then uses these decomposed unitary matrices as pre-filters and post-filters at the transmitter and receiver to achieve near capacity.

In MIMO systems, there is a trade-off between the diversity gain and the capacity gain. When channel situation is bad, for decreasing the BER, we have to use the diversity and decrease the symbol rate (but we can use high lever modulation to improve the bit rate). When channel is good, we can increase the symbol rate by transmitting different data on each transmitter antenna and give up a part of the diversity. We have to use the adaptive scheme to choose the best solution according to the channel situation and channel matrix.

\subsubsection{MIMO-OFDM systems}
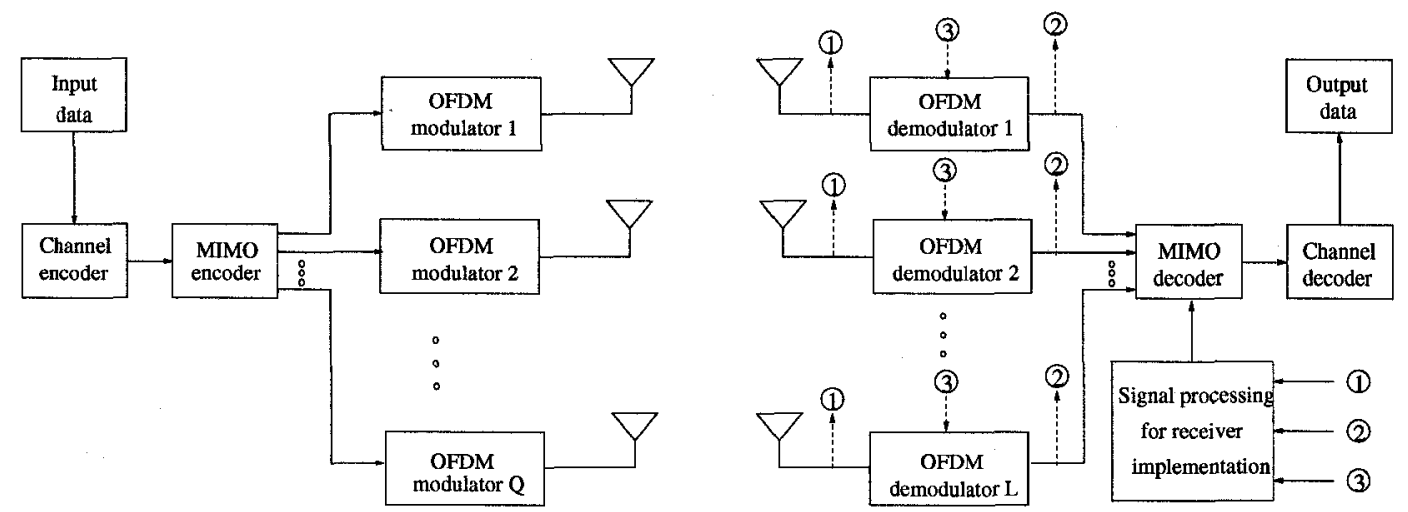

Fig. 2.3 $Q \times$ L MIMO-OFDM system, where $Q$ and $L$ are the numbers of inputs and outputs respectively

When a MIMO system is designed for a frequency selective fading channel, to reduce the equalization complexity of the MIMO system, combining the MIMO system with OFDM modulation would be a good choice [11]. The system becomes the MIMOOFDM system. In Fig. 2.3, we give a block diagram of a MIMO-OFDM system [13]. All MIMO-OFDM receivers must perform time synchronization, carrier and 
sampling frequency offset estimation, and correction and parameter estimation. At the beginning of the frame, we use a preamble containing one or more training sequences. Once the acquisition phase is over, receiver goes into the tracking mode by using training pilots on some subcarriers.

\subsection{System model}

\subsubsection{OFDM system model}

Using the IDFT or IFFT to implement OFDM, from (2.23), the time domain samples of a complex value baseband OFDM symbol can be written as

$$
x_{n}=\frac{1}{\sqrt{N}} \sum_{i=0}^{N-1} X_{i} e^{\frac{j 2 \pi i n}{N}}, n=0,1, \cdots, N-1
$$

where $N$ is IFFT size, $x_{n}$ is the $n^{\text {th }}$ sample of the time domain symbol, $X_{i}$ is the complex modulated data on the $i^{\text {th }}$ subcarrier, and for convenience we use the normalized factor $\frac{1}{\sqrt{N}}$ instead of $\frac{1}{N}$. In a CP-based OFDM system, a CP which is the copy of the last part of the current symbol is added to each OFDM symbol. The signal then is passed through a window and a D/A converter, upconverted to RF, and transmitted. When the CP length is longer than the channel delay spread, the ISI caused by multipath fading is eliminated. At the receiver the signal is downconverted into a baseband signal $\tilde{r}(t)$, passed through a A/D converter, removed of its windowing and sampled at a rate $T_{s a}=T_{F F T} / N$, where $T_{s a}$ is the sample duration. Taking into account the phase shift introduced by a CFO and a timing offset, the received sample can be written as (see (2.44) in section 2.3.4)

$$
r_{k}=s_{k-n_{\epsilon}} e^{j\left(2 \pi \gamma k / N+\theta_{0}\right)}+n_{k}
$$


where $n_{\epsilon}$ is an integer representing the unknown arrival time of a symbol, $\gamma$ is the CFO normalized by the subcarrier spacing, $\theta_{0}$ is the initial phase, $n_{k}$ is the $k^{\text {th }}$ sample of the complex Gaussian noise with zero mean and variance $\sigma_{n}^{2}$. For AWGN channels $s_{k}=x_{k}$. For ISI or fading channels, we use a tapped delay line model introduced in section 2.1.4 and $s_{k}$ is given by

$$
s_{k}=\sum_{m=0}^{M-1} h_{m} x_{k-m}
$$

where $M$ is channel length and $h_{m}$ is the complex baseband channel impulse response at lag $m$.

\subsubsection{MIMO-OFDM system model}

Let us consider the $Q \times L$ space time system in Fig. 2.4 [14].

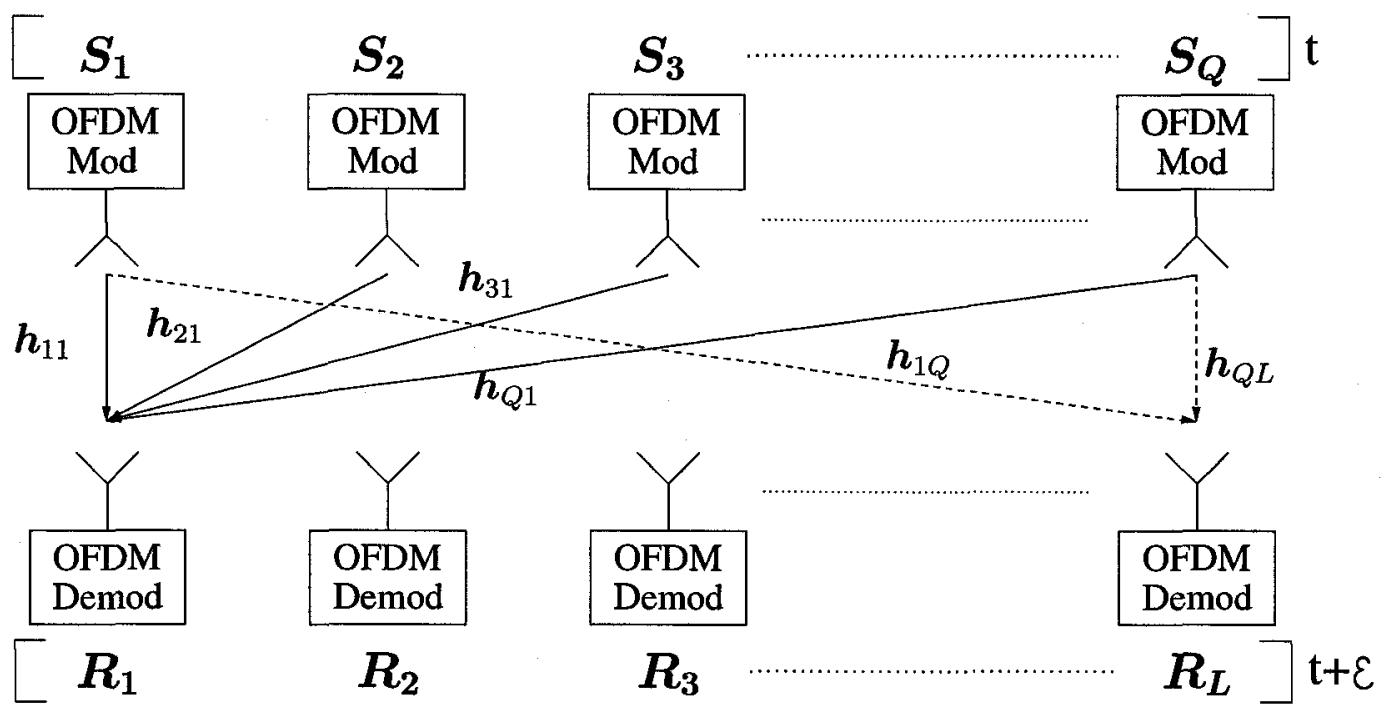

Fig. 2.4 Block diagram of a system with $\mathrm{Q} \times \mathrm{L}$ transmit-receive diversity

Such system has a diversity order of $Q \times L$, Let $S_{q}=\left[S_{q, 0}, \ldots, S_{q, N-1}\right]^{T}$ be the transmitted OFDM symbol on the $q^{\text {th }}$ transmitter antenna, $\boldsymbol{R}_{l}=\left[R_{l, 0}, \ldots, R_{l, N-1}\right]^{T}$ 
be the received OFDM symbol from the $l^{\text {th }}$ receiver antenna. Similar as (2.48), the $N$ point IDFT output sequence for the OFDM symbol $\boldsymbol{S}_{\boldsymbol{q}}$ is given by

$$
s_{q, n}=\frac{1}{\sqrt{N}} \sum_{k=0}^{N-1} S_{q, k} e^{\frac{j 2 \pi n k}{N}}, n=0,1, \cdots, N-1
$$

where $S_{q, k}$ is the transmitted data on the $k^{\text {th }}$ subcarrier from the $q^{\text {th }}$ transmitter antenna and $s_{q, n}$ is the $n^{\text {th }}$ time domain sample from the $q^{\text {th }}$ transmitter antenna. For a fair comparison with SISO systems, the summation of all transmitter antennas power are normalized to be one and we have

$$
\sum_{q=1}^{Q} \sum_{n=0}^{N-1}\left|s_{q, n}\right|^{2}=\sum_{n=0}^{N-1}\left|x_{n}\right|^{2}=1
$$

At the receiver, for the $l^{\text {th }}$ receiver antenna, after removing the windowing and CP, the received sample sequence is

$$
r_{l, k}=\sum_{q=1}^{Q} \sum_{m=0}^{M-1} h_{q l, m} s_{q, k-n_{\epsilon}-m} e^{j\left(2 \pi \gamma k / N+\theta_{0}\right)}+n_{l, k}
$$

where $h_{q l, m}$ is the complex baseband channel impulse response at lag $m$ between the $q^{\text {th }}$ transmitter antenna and the $l^{\text {th }}$ receiver antenna $\left(\boldsymbol{h}_{q, l}=\left[h_{q l, 0}, \ldots, h_{q l, M-1}\right]\right)$ and $M$ is the length of the channel. $n_{\epsilon}$ is an integer representing the unknown arrival time of a symbol, $\gamma$ is the CFO normalized by the subcarrier spacing, $\theta_{0}$ is the initial phase, $n_{l, k}$ is the $k^{t h}$ sample of the complex Gaussian noise with zero mean and variance $\sigma_{n}^{2}$ at the $l^{\text {th }}$ receiver antenna. 


\section{Chapter 3}

\section{Existing synchronization methods}

\subsection{Synchronization methods using training symbols}

\subsubsection{Schmidl and Cox (SC)'s method [6]}

The authors use two training symbols to estimate the symbol timing and carrier frequency offset. The first training symbol has two identical halves in the time domain. The training symbol is a PN sequence on the even subcarriers and zeroes on the odd subcarriers, so that the receiver can separate the training symbol and the data symbol. The first half is identical to the second half after passing the channel, except for a phase shift caused by the carrier frequency offset.

If the conjugate of a sample from the first half is multiplied by the corresponding sample from the second half ( $\frac{T_{F F T}}{2}$ seconds later), the effect of the channel should be canceled out. Only a phase difference of $\phi=\pi \gamma$ will remain, we can use it to estimate the normalized carrier frequency offset $\gamma$. The phase difference is constant because the length between the samples $L_{s c}$ is constant.

In order to increase the frequency capture range, the second training symbol is used. The second training symbol contains a PN sequence on the odd subcarriers 
to measure these subchannels, and another PN sequence on even subcarriers to help determining the CFO.

At the start of the frame, the products of each of these pairs of samples will have almost the same phase, so the magnitude of the sum will be a large value. The sum of products can be expressed in the following equation:

$$
P_{S C}(d)=\sum_{m=0}^{L_{s c}-1}\left(r_{d+m}^{*} \cdot r_{d+m+L_{s c}}\right)
$$

where $L_{s c}=\frac{N}{2}$ samples. $P_{S C}(d)$ can be calculated iteratively,

$$
P_{S C}(d+1)=P_{S C}(d)+r_{d+L_{s c}}^{*} r_{d+2 L_{s c}}-r_{d}^{*} r_{d+L_{s c}}
$$

The energy of the second half of the first training symbol to normalize the sum of the correlation value is used. The energy is calculated as:

$$
R_{S C}(d)=\sum_{m=0}^{L_{s c-1}}\left|r_{d+m+L_{s c}}\right|^{2}
$$

A timing metric is defined as the normalized sum of products. It is:

$$
M_{S C}(d)=\frac{\left|P_{S C}(d)\right|^{2}}{R_{S C}^{2}(d)}
$$

The timing metric exhibits a plateau of length of the guard interval where the metric reaches a maximum when the samples are pairs with distances of half a symbol period. The start of the frame can be taken to be anywhere within this window without a loss in the received SNR. When the signal is propagating over a multipath fading channel, the length of the plateau will be shortened by the length of the channel delay time. This plateau leads to some uncertainty as to the start of the frame, as it will always 
be a "rough" estimation of the symbol timing error. Authors propose an averaging method which find two points with $90 \%$ of the maximum value, one to the left and the other to the right of the maximum point. The timing estimate is taken as the average of the two $90 \%$ points.

We can use the main difference between the two halves of the first training symbol which is a phase difference to estimate the carrier frequency offset.

$$
\begin{gathered}
\phi=\pi \gamma \\
\widehat{\phi}=\angle P_{S C}\left(\widehat{d}_{S C}\right)
\end{gathered}
$$

where $\angle$ denotes the argument of a complex number. $\widehat{d}_{S C}$ is the estimated symbol start point using SC's method. Since (3.6) yields an estimate of $\phi$ only up to a multiple of $2 \pi$, only the fraction part of the CFO can be estimated from $\widehat{\phi}$ as

$$
\widehat{\gamma}_{f}=\frac{\widehat{\phi}}{\pi}
$$

The actual frequency offset is

$$
\gamma=\gamma_{f}+2 g
$$

where $g$ is an integer (possibly equal to 0 ), and $\gamma_{f}$ is the fraction part of the carrier frequency offset. We have to use the second training symbol to estimate $g$. The second training symbol contains a PN sequence modulated differentially with respect to the first training symbol and it can be retrieved and compared with the reference sequence. After the fraction carrier frequency offset correction (by multiplying the sample $r_{n}$, given by (2.49), by $e^{-j 2 \pi n \widehat{\gamma f} / N}$ ), we obtain the corrected samples corresponding to the 
two training symbols as:

$$
\begin{array}{rc}
r_{n} e^{-j 2 \pi n \widehat{\gamma}_{f} / N} & n=0, \ldots, N-1 \\
r_{n+N+g} e^{-j 2 \pi(n+N+g) \widehat{\gamma}_{f} / N} & n=0, \ldots, N-1
\end{array}
$$

Let $x_{1, k}$ and $x_{2, k}$ be the FFT's of $r_{n} e^{-j 2 \pi n \hat{\gamma}_{f} / N}$ and $r_{n+N+g} e^{-j 2 \pi(n+N+g) \hat{\gamma}_{f} / N}$, and let $v_{k}$ be the differentially modulated PN sequence on the even frequencies of the two training symbols.

$$
v_{k}=X_{2, k} / X_{1, k} \quad k=0,2, \cdots, N-2
$$

where $X_{1, k}$ and $X_{2, k}$ are frequency training sequences of first and second training symbol respectively and $k$ is index of the subcarriers. The number of the even positions shifted can be calculated by finding $\widehat{g}$ that maximizes

$$
B(g)=\frac{\left|\sum_{k \in \boldsymbol{X}} x_{1, k+2 g}^{*} v_{k}^{*} x_{2, k+2 g}\right|^{2}}{2\left(\sum_{k \in \boldsymbol{X}}\left|x_{2, k}\right|^{2}\right)^{2}}
$$

where $\boldsymbol{X}$ is the set of indices for the even frequency components given by $\boldsymbol{X}=$ $\{0,2, \cdots, N-2\}$. The carrier frequency offset estimate is obtained as

$$
\widehat{\gamma}=\frac{\widehat{\phi}}{\pi}+2 \widehat{g}
$$

We can also use the second training symbol to estimate the channel.

\subsubsection{Minn's method [7]}

First, Minn used

$$
R_{f}(d)=\frac{1}{2} \sum_{m=0}^{N-1}\left|r_{d+m}\right|^{2}
$$


to replace the $R_{S C}(d)$ in (3.4), which decreases the variance of the symbol timing estimator. Then he proposed the new training pattern $\left[\begin{array}{lll}A & A-A & -A\end{array}\right]$ where $A$ represents samples of length $L_{M}=N / 4$ generated by $N / 4$ point IFFT of $N_{u} / 4$ length modulated data of a PN sequence ( $N_{u}$ is the number of used subcarriers). The timing metric is given by

$$
M_{\text {Minn }}(d)=\frac{\left|P_{\text {Minn }}(d)\right|^{2}}{R_{\text {Minn }}^{2}(d)}
$$

where

$$
P_{M i n n}(d)=\sum_{k=0}^{1} \sum_{m=0}^{L_{M}-1} r_{d+2 k L_{M}+m}^{*} \cdot r_{d+2 k L_{M}+m+L_{M}}
$$

and

$$
R_{M i n n}(d)=\sum_{k=0}^{1} \sum_{m=0}^{L_{M}-1}\left|r_{d+2 k L_{M}+m+L_{M}}\right|^{2}
$$

The estimated start point of the symbol is

$$
\widehat{d}_{M i n n}=\arg \underbrace{\max }_{d}\left\{M_{M i n n}(d)\right\}
$$

When we use $R_{f}(d)$ in (3.12) to replace $R_{\text {Minn }}(d)$ in (3.13), we can also decrease the variance of the timing estimator obtained with Minn's training pattern.

Using Minn's method, the timing metric does not have the plateau any more, so the uncertainty of the timing estimation has been reduced. It works well in an AWGN channel, but in fading channels, due to the multipath effect, the mean value will not be in the exact start point and will shift to the right. It introduces large ISI since the FFT will include some samples which are from the next symbol.

For carrier frequency offset estimation, it uses the repeated parts length which is the half of the SC's method. Since the variance of the frequency estimator depends on the time error, SNR, and length of the training symbol, it has a little degradation 
[8]. The estimated fraction part of $\mathrm{CFO}$ is

$$
\widehat{\gamma_{f}}=\frac{2}{\pi} \angle P_{M i n n}\left(\widehat{d}_{M i n n}\right)
$$

where $\widehat{d}_{\text {Minn }}$ is the estimated symbol start point using Minn's method. Note that the ' 2 ' in (3.17) comes from the fact that the length of the repeated part of the training symbol in Minn's method is half SC'one. Minn did not propose any method for estimating the integer part of the CFO.

\subsection{Synchronization methods for MIMO-OFDM systems}

Similar to OFDM systems, MIMO-OFDM systems are also very sensitive to synchronization errors. Therefore, we have to design robust synchronization methods for them. Since multiple antennas are used, we can achieve better performance of the synchronization from the spatial diversity. In [13] [14] and [15], authors describe a synchronization method in the acquisition mode by four steps. The system model is same as the model described in section 2.5.2. The frame structure is given in Fig. 3.1.

\section{Antenna 1}

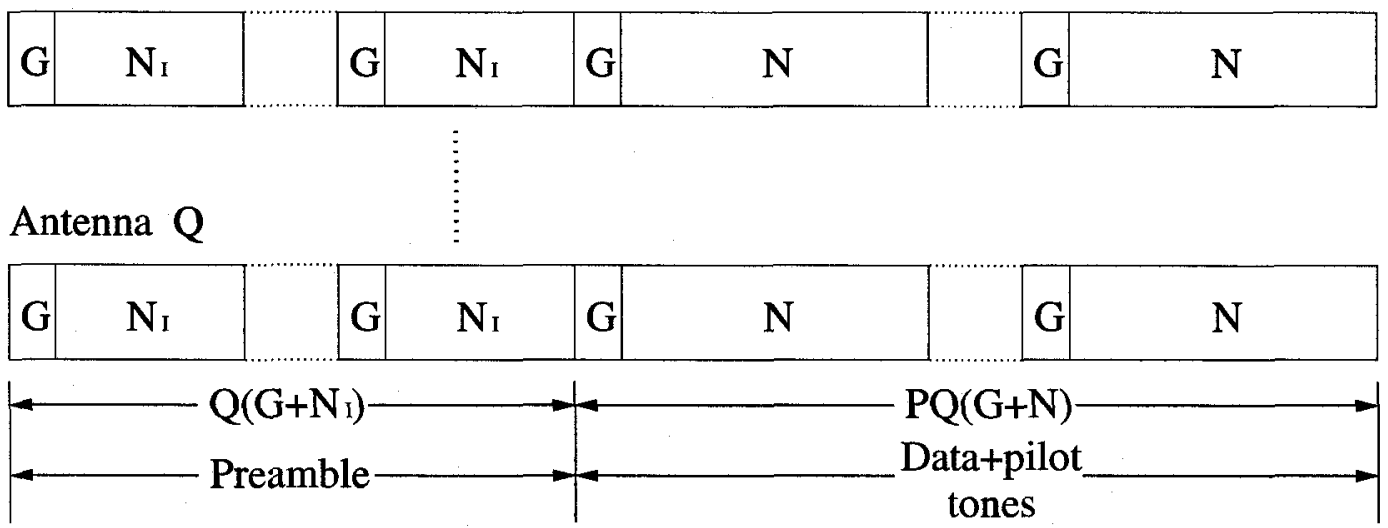

Fig. 3.1 Frame structure for the $\mathrm{Q} \times \mathrm{L}$ MIMO-OFDM system 
where $G$ is the CP length, $N_{I}$ is the length of the short training symbol, $N$ is the length of the data symbol, $Q$ is the number of transmitter antennas.

- Step 1) Coarse Time Synchronization and Signal Detection

Due to the CP (or guard interval), coarse time acquisition can be performed by autocorrelating the received, down converted discretized complex samples over a window of $\mathrm{G}$ samples that are at a distance of $N_{I}$ from each other over a length $G$ window:

$$
n_{l, \text { coarse }}=\arg \underbrace{\max }_{n}\left\{\phi_{l, n}\right\}
$$

where

$$
\phi_{l, n}=\sum_{k=0}^{G-1}\left(r_{l, n+k}^{*} \cdot r_{l, n+k+N_{I}}\right)
$$

where $r_{l, k}$ is the $k^{\text {th }}$ received sample at the $l^{\text {th }}$ receiver antenna (given by (2.53)) and maximization of $\phi_{l, n}$ is performed only if $\phi_{l, n}$ exceeds a certain threshold which is $10 \%$ of the incoming signal energy contained in the correlation window.

- Step 2) Frequency Offset Estimation in the Time Domain

Let $\gamma$ be the carrier frequency offset between the transmitter and receiver local oscillators normalized by the subcarrier spacing. The samples at a distance of $N_{I}$ in the time domain have a phase shift $\phi=2 \pi \gamma N_{I} / N$. The frequency offset estimate of up to $\pm I / 2$ subcarrier spacings is

$$
\widehat{\gamma}_{f, l}=\frac{I}{2 \pi} \angle\left\{\phi_{n_{l, \text { coarse }}}\right\}
$$

where $I=N / N_{I}$. By reducing the length of the training symbol by a factor of $I$, we increase the range of the frequency offset estimate by a factor of $I$. If we 
multiply the received sample sequence with $e^{-j 2 \pi \hat{\gamma} k / N_{I}}$ during the preamble and $e^{-j 2 \pi \hat{\gamma} k / N}$ during the data portion, the fraction part of the CFO can be removed.

- Step 3) Residual Frequency Offset Correction

If the range of the $\mathrm{CFO}$ is over $\pm I / 2$ subcarrier spacings, frequency domain processing can be used. Let $\left\{S_{q, k}\right\}_{k=0}^{N-1}$ be the frequency domain training sequence, obtained by taking $N$ point FFT of the time domain training sequence $\left\{s_{q, n}\right\}_{n=0}^{N_{I}-1}$ transmitted from the $q^{\text {th }}$ transmitter antenna. Suppose that the same frequency domain training sequence $\left\{S_{q, k}\right\}_{k=0}^{N-1}$ is transmitted from all the transmitter antennas. The residual frequency offset can be estimated by taking a cyclic cross-correlation of $\left\{S_{q, k}\right\}_{k=0}^{N-1}$ with the received, frequency corrected and demodulated symbol sequence $R_{l, n}^{c}=F F T_{N}\left\{r_{l, k} e^{-j 2 \pi \hat{\gamma}_{f, l} k / N_{I}}\right\}$ and we have ${ }^{1}$

$$
\chi_{l, k}=\sum_{n=0}^{N-1} S_{q,(k+n)_{N}}^{*} R_{l, n}^{c} \quad k=0,1, \cdots, N-1
$$

where $(k+n)_{N}$ denotes $k+n \bmod N$. The integer frequency offset is estimated as

$$
\widehat{g}_{l}=\arg \max \left\{\left|\chi_{l, k}\right|\right\} \quad k=0,1, \cdots, N-1
$$

The overall CFO estimate is then obtained as

$$
\widehat{\gamma}_{l}=\widehat{\gamma}_{f, l}+\widehat{g}_{l}
$$

- Step 4) Fine Time Synchronization

When the frequency offset is removed, fine time synchronization can be performed by cross-correlating the received, frequency corrected samples with the

\footnotetext{
${ }^{1}$ Note that $\chi_{l, k}$ does not depend on $q$ since it is assumed the same frequency domain training sequence is used for all transmitter antennas.
} 
transmitted sequences. The general fine time synchronization metric can be given by:

$$
n_{l, f i n e}=\arg \underbrace{\max }_{n}\left\{\psi_{l, n}\right\}
$$

where

$$
\psi_{l, n}=\sum_{q=1}^{Q} \sum_{k=0}^{N_{I}-1}\left(s_{q, k}^{*} r_{l, n+k}^{c}\right)
$$

and

$$
r_{l, k}^{c}=r_{l, k} e^{-j 2 \pi \widehat{\gamma}_{l} k / N_{I}}
$$

If the training sequences are orthogonal with each other, only one cross-correlation is needed per receiver antenna. The threshold is set at $10 \%$ of the energy contained in $N_{I}$ received samples. Since it is a computationally consuming process, we should use a small window which is centered around the coarse time estimated point $n_{l, \text { coarse }}$. Finally, the estimated start point is $n_{\text {opt }}=\frac{1}{L} \sum_{l=1}^{L} n_{l, \text { fine }}$.

From the frame structure, we know the preamble is used not only for synchronization but also for channel estimation. The preamble is efficient, but this method also has some drawbacks. First, most of MIMO-OFDM channels are frequency selective fading channels, i.e. each subcarrier has a different frequency domain channel coefficient. In step 3, the cyclic cross-correlation would be affected by different channel coefficients and it makes the estimation of the residual frequency offset inaccurate. It also affects the performance of fine time synchronization. Secondly, in step 4, the threshold which is set at $10 \%$ of the energy contained in $N_{I}$ received samples is chosen independently of SNR. And no theoretical support is provided for the choice of the threshold. Finally, $[13,14,15]$ do not clearly explain how to use spatial diversity.

Unlike [13] [14] and [15], [12] and [24] focus on the spatial diversity for the synchronization in MIMO-OFDM systems. In [12], the author extends the SC's method from 
OFDM systems to MIMO-OFDM systems. It uses a preamble containing two identical parts without CP. The length of the preamble is $N$. We assume $Q$ transmitter antennas and $L$ receiver antennas are used. The timing metric is given by:

$$
P(d)=\sum_{i=0}^{L-1} w_{i} P_{i}(d)
$$

where $w_{i}$ is the combining weight. If $w_{i}=1$, the system implements equal gain combining (EGC). If $w_{i}=\max \left(\left|P_{i}(d)\right|\right)$, the system is under a maximum ratio combining (MRC).

$$
P_{i}(d)=\sum_{m=0}^{N / 2-1} r_{i, d+m}^{*} r_{i, d+m+N / 2}
$$

where $r_{i, k}$ is the $k^{\text {th }}$ received sample at the $i^{\text {th }}$ receiver antenna.

The estimated start point of the symbol is

$$
\widehat{d}_{M V C}=\arg \underbrace{\max }_{d}\{P(d)\}
$$

The estimated fraction part of the CFO is given by:

$$
\widehat{\gamma_{f}}=\frac{\angle P\left(\widehat{d}_{M V C}\right)}{\pi}
$$

From the simulation, the author concluded that MRC had better performance than EGC. There are two drawbacks of this method, one is that no method is given for estimating the integer part of the CFO, the other is that the estimated start point is not accurate in multipath channels and shifted to the right side of the exact start point. 


\section{Chapter 4}

\section{Proposed synchronization method for OFDM and MIMO-OFDM} systems

\subsection{Proposed synchronization method for OFDM systems}

\subsubsection{The training symbols structure of the proposed method}

As seen from (3.5) and (3.6), the fraction CFO is estimated from the angle of $P_{S C}$ at the estimated start point, so the CFO estimator's performance depends on the timing estimator's performance and an accurate timing estimator is essential.

As explained in section 3.1.1, CP causes timing metric uncertainty. Furthermore, since the first symbol contains two identical parts, to estimate the CFO integer part we can perform an $N / 2$ point FFT on only the second half symbol in order to get the training data on even subcarriers. Therefore, having ISI in that symbol will not have an impact on the estimation of the CFO integer part. Since we are only using the time domain signals to do the estimation of the symbol timing and the fraction part 
of the CFO, we can remove the CP from the first training symbol.

The proposed method uses two training symbols to do the time and carrier frequency synchronization. The first training symbol is used for estimating the coarse time and the CFO fraction part. The first symbol pattern of the proposed synchronization method for AWGN channels is illustrated in Fig. 4.1 b. For the proposed method, the first OFDM symbol contains two identical parts $[\boldsymbol{a} a]$ which are Chu sequences with length $L_{p}=N / 2$. Chu sequences are chosen because they are constant envelop sequences with a perfect autocorrelation function [25]. All circular autocorrelations are zero except for the circular shift $D$, when $\bmod \left(D / L_{p}\right)=0$, where $L_{p}$ is the length of sequences.

\begin{tabular}{|c|c|c|}
\hline Cyclic Prefix & Identical Part $\boldsymbol{a}$ & Identical Part $\boldsymbol{a}$ \\
$G$ & $N / 2$ & $N / 2$ \\
\hline
\end{tabular}

(a) SC's method first symbol

\begin{tabular}{|c|c|c|c|}
\hline FCP & Identical Part $\boldsymbol{a}$ & Identical Part $\boldsymbol{a}$ & FlP \\
$G / 2$ & $N / 2$ & $N / 2$ & $G / 2$ \\
\hline
\end{tabular}

(b) SISO proposed method first symbol in AWGN channels

\begin{tabular}{|c|c|c|r|c|c|}
\hline ZPP & Identical Part $\quad \boldsymbol{a}_{\text {sh }}$ & 0 & Identical Part $\boldsymbol{a}_{\text {sh }}$ & 0 & FIP \\
$G / 2$ & $N / 2-G / 2$ & $G / 2$ & $N / 2-G / 2$ & $G / 2$ & $G / 2$ \\
\hline
\end{tabular}

(c) SISO proposed method first symbol in ISI or fading channels

\begin{tabular}{|l|c|c|c|}
\hline $\mathrm{CP}$ & Identical Part & Identical Part & FIP \\
$G / 2$ & $\boldsymbol{a}_{q, 0}=\boldsymbol{a}_{1,(q-1) G}$ & $\boldsymbol{a}_{q, 0}=\boldsymbol{a}_{1,(q-1) G}$ & $N / 2$ \\
$N / 2$ & $N / 2$ \\
\hline
\end{tabular}

(d) MIMO proposed method first symbol in fading channels ( $q^{\text {th }} \mathrm{Tx}$ antenna)

Fig. 4.1 First symbol structure of proposed method and SC's method

In addition to the two identical parts, in an AWGN channel, we add a Flipped cyclic prefix (FCP) and a Flipped Postfix (FIP) for the first training symbol (see 


\section{Proposed synchronization method for OFDM and MIMO-OFDM} systems

Fig. 4.1 b.). The FCP is the negative copy of the last $G / 2$ samples of $\boldsymbol{a}$ and FIP is the negative copy of the first $G / 2$ samples of $\boldsymbol{a}$, where $G$ is the number of the CP samples. Let $\boldsymbol{a}$ be $\left[a_{0}, a_{1}, \cdots, a_{N / 2-1}\right]$. The first symbol $\boldsymbol{x}_{\text {preamble }}$ in an AWGN channel is

$$
\left[-a_{\frac{N-G}{2}}, \cdots,-a_{\frac{N}{2}-1}, a_{0}, a_{1}, \cdots, a_{\frac{N}{2}-1}, a_{0}, a_{1}, \cdots, a_{\frac{N}{2}-1},-a_{0},-a_{1}, \cdots,-a_{\frac{G}{2}-1}\right]
$$

In fading channels, multipath will cause the estimated start point to shift to the right side of the exact start point. Recalling the tapped delay line model in (2.49)-(2.50), the timing metric would be the summation of each tap timing metrics, hence the right shift. Usually, most of the channel power is located in first few taps (e.g. channel with exponential decaying power delay profile). To make the timing estimator robust in fading or ISI channels, we generate each half training sequences using Chu sequences with length $\frac{N}{2}-\frac{G}{2}\left(\boldsymbol{a}_{\mathrm{sh}}\right.$, where 'sh' stands for short) and pad $\frac{G}{2}$ zeroes at each half end (see Fig. 4.1 c.). Such proposed preamble works well in burst communication scenarios, but in continuous communication scenarios, first several samples will be corrupted by the previous symbol which makes the estimated start point shift to the right. To make the timing estimator robust in continuous communication scenarios as well, the length of FIP was chosen to be $G / 2$ samples instead of choosing $G$ (SC method CP length) and we add a $G / 2$ zero prefix at the beginning of the symbol (see Fig. $4.1 \mathrm{c}$.$) .$

The second training symbol is used for estimating the CFO integer part and performing the fine time synchronization. It is a known Chu sequence with length $N$. Since the Chu sequences for the two training symbols are known and of different lengths, the CFO integer part can be estimated by using the differences of the two training symbols on the even frequencies. 


\subsubsection{Implementing the proposed synchronization method}

The proposed method can be implemented in four steps:

- Step 1) Coarse Time Synchronization

The proposed method uses the timing metric similar as SC's method. Let $r_{k}$ be the complex samples taken at the output of A/D (see Fig. 2.2), given by (2.49). Since the first symbol has two identical parts, after passing a channel, the first part is same as the second part except for a phase shift by the CFO. If the conjugate of a sample from the first half is multiplied by the corresponding sample from the second half, the effect of channel is canceled out. At the start of the frame, the products of each of these pairs of samples will have almost same phase, so the magnitude of the sum will be a large value. The sum of products can be expressed in the following equation:

$$
P_{p}(d)=\sum_{m=0}^{L_{p}-1}\left(r_{d+m}^{*} \cdot r_{d+m+L_{p}}\right)
$$

where $L_{p}=\frac{N}{2}$ samples. We will use the energy of the second half of the first training symbol to normalize the sum of the correlation value. The energy is calculated as:

$$
R_{p}(d)=\sum_{m=0}^{L_{p}-1}\left|r_{d+m+L_{p}}\right|^{2}
$$

A timing metric is defined as the normalized sum of products. It is:

$$
M_{p}(d)=\frac{\left|P_{p}(d)\right|^{2}}{R_{p}^{2}(d)}
$$

If we adopt Minn's modified $R_{f}(d)$ given in (3.12) instead of $R_{p}(d)$ in (4.3), the variance of the timing estimator decreases at the expense of a slight increase 
in complexity. The coarse estimation of the start point is taken at the peak of timing metric and it is given by

$$
\widehat{d}_{\text {coarse }}=\arg \underbrace{\max }_{d}\left\{M_{p}(d)\right\}
$$

- Step 2) Estimating the Fraction Part of the CFO

At the start of the frame, the products of each of these pairs of samples will have approximately same phase $\phi=\pi \gamma$, where $\gamma$ is the CFO normalized by the subcarrier spacing. We can use the phase of those products summation to estimate the carrier frequency offset. Let

$$
\widehat{\phi}=\angle P_{p}\left(\widehat{d}_{c o a r s e}\right)
$$

Since (4.5) yields an estimate of $\phi$ only up to a multiple of $2 \pi$, only the fraction part of the CFO can be estimated from $\widehat{\phi}$ as

$$
\widehat{\gamma}_{f}=\frac{\widehat{\phi}}{\pi}
$$

The actual frequency offset is

$$
\gamma=\gamma_{f}+2 g
$$

where $g$ is an integer (possibly null) and $\gamma_{f}$ is the fraction part of the carrier frequency offset. The estimated fraction part of the CFO is

$$
\widehat{\gamma_{f}}=\frac{\widehat{\phi}}{\pi}=\frac{\angle P_{p}\left(\widehat{d}_{\text {coarse }}\right)}{\pi}
$$




\section{Proposed synchronization method for OFDM and MIMO-OFDM}

In this step, the frequency offset estimation is up to \pm 1 subcarrier spacing. To remove the fraction part of the CFO, we should multiply the received sample sequences $r_{k}$, given by (2.49) with $e^{-j 2 \pi \widehat{\gamma_{f}} k / N}$.

- Step 3) Estimating the Integer Part of the CFO

To estimate the integer part of the $\mathrm{CFO}$, we have to use the second training symbol. The second training symbol contains a known sequence on even subcarriers.

Since we did not use CP in the first training symbol, property of the circular convolution with the channel coefficients is not retained any more. But the first symbol contains two identical parts, only even subcarriers contain the information and all odd subcarriers are zeroes. The second part of the first training symbol retains property of the circular convolution with the channel coefficients. To estimate the CFO integer part we perform an $N / 2$ point FFT on the second half of the first symbol in order to get the data on even subcarriers.

After the fraction CFO correction (by multiplying the samples $r_{k}$ by $e^{-j 2 \pi \widehat{\gamma_{f}} k / N}$ ), we obtain the corrected samples corresponding to the two training symbols as:

$$
\begin{array}{rl}
r_{n} e^{-j 2 \pi n \widehat{\gamma}_{f} / N} & n=0, \ldots, N-1 \\
r_{n+N+g} e^{-j 2 \pi(n+N+g) \widehat{\gamma}_{f} / N} & n=0, \ldots, N-1
\end{array}
$$

Let $x_{1, k}$ and $x_{2, k}$ be the FFT's of $r_{n} e^{-j 2 \pi n \widehat{\gamma}_{f} / N}$ and $r_{n+N+g} e^{-j 2 \pi(n+N+g) \widehat{\gamma}_{f} / N}$. Let $\left\{c_{k}\right\}$ be the ratio sequence on the even frequencies of the two training symbols.

$$
c_{k}=X_{1, k} / X_{2, k} \quad k=0,2, \cdots, N-2
$$


where $X_{1, k}$ and $X_{2, k}$ are frequency training sequences of the first and second training symbol respectively and $k$ is the index of the subcarrier. The number of the even positions shifted can be calculated by finding $\widehat{g}$ that maximizes

$$
G(g)=\frac{\left|\sum_{k \in \boldsymbol{X}} x_{2, k+2 g}^{*} c_{k}^{*} x_{1, k+2 g}\right|^{2}}{2\left(\sum_{k \in X}\left|x_{1, k}\right|^{2}\right)^{2}}
$$

where $\boldsymbol{X}$ is the set of indices for the even frequency components and $\boldsymbol{X}=$ $\{0,2, \cdots, N-2\}$. The estimated integer part of the CFO is

$$
\widehat{g}=\arg \underbrace{\max }_{g}\{G(g)\}
$$

The estimated carrier frequency offset is obtained as

$$
\widehat{\gamma}=\widehat{\gamma_{f}}+2 \widehat{g}
$$

where $\widehat{\gamma_{f}}$ is given by (4.8). If we compare (4.9) and (4.10) with (3.9) and (3.10), we can find a slight difference of definitions of $c_{k}$ and $v_{k}$. Since we add $G$ zeroes in the first training symbol under ISI or fading channels, the frequency domain training sequence $X_{1, k}$ would not be a constant envelop sequence. The sample values on some subcarriers might be small. To reduce the effect of large value $v_{k}$ to $B(g)$, we use the $c_{k}$ and $G(g)$ instead of the $v_{k}$ and $B(g)$.

If we multiply the received sample sequence $r_{k}$ with $e^{-j 2 \pi \widehat{\gamma} k / N}$, the CFO is removed.

- Step 4) Fine Time Synchronization

If the accuracy of the coarse time synchronization is not enough for the system demand, the fine time synchronization method can be used to achieve better 
performance for the timing estimation. The price to pay is the computation complexity. First, we have to remove the $\mathrm{CFO}$ effect by multiplying the received sample sequence $r_{k}$ with $e^{-j 2 \pi \widehat{\gamma} k / N}$. The carrier frequency corrected time domain sample is

$$
r_{k}^{c}=r_{k} e^{-j 2 \pi \gamma k / N}
$$

Secondly, we calculate the cross-correlation of the carrier frequency corrected sequences $\left\{r_{k}^{c}\right\}$ and the transmitted preamble sequences. Since the first training symbol does not have a cyclic prefix, we use the cross-correlation function of the carrier frequency corrected sequences $\left\{r_{k}^{c}\right\}$ and the second time training sequences excluding $\mathrm{CP}\left\{b_{k}\right\}$ obtained as follows:

$$
\psi_{n}=\sum_{k=0}^{N-1} b_{k}^{*} r_{n+k}^{c}, n \in\left[-G / 2+\widehat{d}_{c o a r s e}+N+1.5 G, \widehat{d}_{c o a r s e}+N+1.5 G+G / 2\right]
$$

From the central limit theorem (CLT), $\psi_{n}$ is a Gaussian RV. We can also use the cross correlation of the carrier frequency corrected sequences $\left\{r_{k}^{c}\right\}$ and two training sequences excluding prefix and postfix by length $2 N$ (In that later case, we have to remove $G$ samples in the middle of $\left.\left\{r_{k}^{c}\right\}\right)$. The accuracy of timing estimation and complexity would be increased. In this thesis, since one symbol length cross correlation is enough for the system demand, we only use one symbol length cross-correlation to do the fine time estimation. All analysis and simulations consider this situation.

To get $\widehat{d}_{\text {fine }}$, we search the first point $\widehat{n}$ in $\left[-G / 2+\widehat{d}_{\text {coarse }}+N+1.5 G, \widehat{d}_{\text {coarse }}+N+\right.$ $1.5 G+G / 2]$ (from smallest to greatest) whose $\left|\psi_{\hat{n}}\right|$ is greater than a threshold 
$\eta$. Then we obtain fine time synchronization using

$$
\widehat{d}_{\text {fine }}=\widehat{n}-N-1.5 G
$$

Appendix B presents the methodology how to set the threshold for the fine time synchronization. From appendix B, we set

$$
\begin{aligned}
\eta_{A W G N}= & R_{f}\left(\widehat{d}_{\text {coarse }}\right) \sqrt{M_{p}\left(\widehat{d}_{\text {coarse }}\right)} \\
\eta_{I S I}= & \begin{cases}0.2 R_{f}\left(\widehat{d}_{\text {coarse }}\right) \sqrt{M_{p}\left(\widehat{d}_{\text {coarse }}\right)} & \text { if } \widehat{S N R}>5 d B \\
0.3 R_{f}\left(\widehat{d}_{\text {coarse }}\right) \sqrt{M_{p}\left(\widehat{d}_{\text {coarse }}\right)} & \text { if } \widehat{S N R} \leq 5 d B\end{cases} \\
\eta_{\text {Rayleigh }}= & \begin{cases}0.2 R_{f}\left(\widehat{d}_{\text {coarse }}\right) \sqrt{M_{p}\left(\widehat{d}_{\text {coarse }}\right)} & \text { if } \widehat{S N R}>5 d B \\
0.3 R_{f}\left(\widehat{d}_{\text {coarse }}\right) \sqrt{M_{p}\left(\widehat{d}_{\text {coarse }}\right)} & \text { if } \widehat{S N R} \leq 5 d B\end{cases}
\end{aligned}
$$

The complexity of the cross-correlation method depends partly on the search

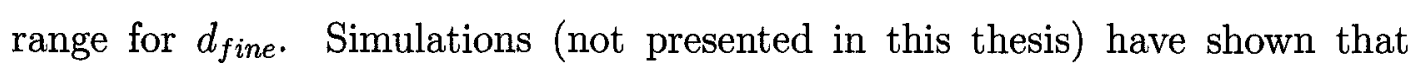
increasing the range beyond $\left[-G / 2+\widehat{d}_{\text {coarse }}, \widehat{d}_{\text {coarse }}+G / 2\right]$ did not improve significantly the accuracy of the timing estimator of the proposed method. Therefore to limit the computational complexity of the method, we propose to search $d_{f i n e}$ in $\left[-G / 2+\widehat{d}_{c o a r s e}, \widehat{d}_{\text {coarse }}+G / 2\right]$. For SC's method, we can also implement the fine time synchronization, but the searching range should be $\left[-G+\widehat{d}_{c o a r s e}^{s c}, \widehat{d}_{\text {coarse }}^{s c}+G\right]$ to get meaningful results. Comparing the range of the two methods, the proposed method has less complexity than SC's method. 


\subsubsection{Analysis of effect of the FIP in an AWGN channel}

For simplicity, we consider an AWGN channel with unmodified $R_{p}(d)$. Analysis can be generalized to ISI or fading channels scenarios with $R_{f}(d)$ given by (3.12). We define the Random Variable (RV)

$$
M_{d}=M_{p}\left(d_{o p t}\right)-M_{p}\left(d_{o p t+1}\right)
$$

where $d_{o p t}$ is the exact symbol start point excluding the prefix and postfix, $d_{o p t+1}$ is the exact symbol start point shifted right by one sample and $M_{p}(d)$ is given in (4.3). Let each complex sample $r_{m}=s_{m}+n_{m}$ be made up of a signal and noise component. Let the variance of the real and imaginary components be $E\left\{\Re\left\{s_{m}\right\}^{2}\right\}=E\left\{\Im\left\{s_{m}\right\}^{2}\right\}=$ $\sigma_{s}^{2} / 2$, and $E\left\{\Re\left\{n_{m}\right\}^{2}\right\}=E\left\{\Im\left\{n_{m}\right\}^{2}\right\}=\sigma_{n}^{2} / 2$, respectively. SNR is $\sigma_{s}^{2} / \sigma_{n}^{2}$. From [6], we know the $\left|P_{p}(d)\right|, R_{p}(d)$, and $M_{p}(d)$ can be approximated by Gaussian RVs due to the central limit theorem. Since $\left|P_{p}(d)\right|$ and $R_{p}(d)$ have much larger means than their variances, $\left|P_{p}(d)\right|^{2}$ and $R_{p}^{2}(d)$ can also be approximated by Gaussian RVs with mean $L_{p}^{2} \sigma_{s}^{4}$, and $L_{p}^{2}\left(\sigma_{s}^{2}+\sigma_{n}^{2}\right)^{2}$ respectively, also quite larger than their variances. Using $(1+b) /(1+c) \approx(1+b-c)$ ( $b$ and $c$ are zero mean Gaussian RVs and their variances are quite smaller than one) we have

$$
\begin{aligned}
M_{p}\left(d_{o p t}\right) & =\frac{\left|P_{p}\left(d_{\text {opt }}\right)\right|^{2}}{R_{p}^{2}\left(d_{o p t}\right)}=\frac{L_{p}^{2} \sigma_{s}^{4}}{L_{p}^{2}\left(\sigma_{s}^{2}+\sigma_{n}^{2}\right)^{2}} \frac{1+\delta P}{1+\delta R} \approx \frac{\sigma_{s}^{4}}{\left(\sigma_{s}^{2}+\sigma_{n}^{2}\right)^{2}}(1+\delta P-\delta R) \\
& =\frac{\left|P_{p}\left(d_{o p t}\right)\right|^{2}}{L_{p}^{2}\left(\sigma_{s}^{2}+\sigma_{n}^{2}\right)^{2}}-\frac{R_{p}^{2}\left(d_{o p t}\right) \sigma_{s}^{4}}{L_{p}^{2}\left(\sigma_{s}^{2}+\sigma_{n}^{2}\right)^{4}}+\frac{\sigma_{s}^{4}}{\left(\sigma_{s}^{2}+\sigma_{n}^{2}\right)^{2}}
\end{aligned}
$$

where $1+\delta P \triangleq\left|P_{p}\left(d_{o p t}\right)\right|^{2} /\left(L_{p}^{2} \sigma_{s}^{4}\right)$ and $1+\delta R \triangleq R_{p}^{2}\left(d_{o p t}\right) /\left(L_{p}^{2}\left(\sigma_{s}^{2}+\sigma_{n}^{2}\right)^{2}\right)$. Similarly

$$
M_{p}\left(d_{o p t+1}\right) \approx \frac{\left|P_{p}\left(d_{o p t+1}\right)\right|^{2}}{L_{p}^{2}\left(\sigma_{s}^{2}+\sigma_{n}^{2}\right)^{2}}-\frac{R_{p}^{2}\left(d_{o p t+1}\right) \sigma_{s}^{4}}{L_{p}^{2}\left(\sigma_{s}^{2}+\sigma_{n}^{2}\right)^{4}}+\frac{\sigma_{s}^{4}}{\left(\sigma_{s}^{2}+\sigma_{n}^{2}\right)^{2}}
$$




\section{Proposed synchronization method for OFDM and MIMO-OFDM} systems

where we have used $L_{p}-2 \approx L_{p}$ and $L_{p}-1 \approx L_{p}$ when $L_{p}$ is large. $M_{p}\left(d_{o p t}\right), M_{p}\left(d_{o p t+1}\right)$ can be approximated by Gaussian RVs, so $M_{d}=M_{p}\left(d_{\text {opt }}\right)-M_{p}\left(d_{\text {opt }+1}\right)$ is also a Gaussian RV given by

$$
M_{d} \approx \frac{\left|P_{p}\left(d_{o p t}\right)\right|^{2}-\left|P_{p}\left(d_{o p t+1}\right)\right|^{2}}{L_{p}^{2}\left(\sigma_{s}^{2}+\sigma_{n}^{2}\right)^{2}}+\frac{\sigma_{s}^{4}}{L_{p}^{2}\left(\sigma_{s}^{2}+\sigma_{n}^{2}\right)^{4}}\left(R_{p}^{2}\left(d_{o p t+1}\right)-R_{p}^{2}\left(d_{o p t}\right)\right)
$$

To calculate the mean and variance of $M_{d}$, we have to calculate the means and variances of $P_{d}=\left|P_{p}\left(d_{\text {opt }}\right)\right|^{2}-\left|P_{p}\left(d_{\text {opt }+1}\right)\right|^{2}$ and $R_{d}=R_{p}^{2}\left(d_{\text {opt }+1}\right)-R_{p}^{2}\left(d_{\text {opt }}\right)$ and their covariance. Let $c_{1}=\frac{1}{L_{p}^{2}\left(\sigma_{s}^{2}+\sigma_{n}^{2}\right)^{2}}$ and $c_{2}=\frac{\sigma_{s}^{4}}{L_{p}^{2}\left(\sigma_{s}^{2}+\sigma_{n}^{2}\right)^{4}}$. The mean of $M_{d}$ is $E\left\{M_{d}\right\}=c_{1} \cdot E\left\{P_{d}\right\}+c_{2} \cdot E\left\{R_{d}\right\}$, From appendix A, we have

$$
E\left\{M_{d}\right\}= \begin{cases}\frac{4\left(L_{p}-1\right) \sigma_{s}^{4}}{L_{p}^{2}\left(\sigma_{s}^{2}+\sigma_{n}^{2}\right)^{2}} & \text { using the FlP preamble. } \\ \frac{2\left(L_{p}-1\right) \sigma_{s}^{4}}{L_{p}^{2}\left(\sigma_{s}^{2}+\sigma_{n}^{2}\right)^{2}} & \text { using SC's preamble. }\end{cases}
$$

and $\operatorname{Var}\left(M_{d}\right)=c_{1}^{2} \cdot \operatorname{Var}\left(P_{d}\right)+c_{2}^{2} \cdot \operatorname{Var}\left(R_{d}\right)+2 \cdot c_{1} \cdot c_{2} \cdot \operatorname{Cov}\left(P_{d}, R_{d}\right)$

$$
=\left\{\begin{array}{l}
\frac{1}{L_{p}^{4}\left(\sigma_{s}^{2}+\sigma_{n}^{2}\right)^{4}}\left[4\left(L_{p}-1\right)^{2}\left(3 \sigma_{s}^{6} \sigma_{n}^{2}+\sigma_{s}^{4} \sigma_{n}^{4}\right)+4\left(L_{p}-1\right)\left(2 \sigma_{s}^{4}+2 \sigma_{s}^{2} \sigma_{n}^{2}+\sigma_{n}^{4}\right)\right. \\
\left.\left(2 \sigma_{s}^{2} \sigma_{n}^{2}+\sigma_{n}^{4}\right)+8\left(\sigma_{s}^{6} \sigma_{n}^{2}+\sigma_{s}^{4} \sigma_{n}^{4}+\sigma_{s}^{2} \sigma_{n}^{6}\right)\right]+\frac{\sigma_{s}^{8}}{L_{p}^{4}\left(\sigma_{s}^{2}+\sigma_{n}^{2}\right)^{8}}\left[16 L_{p}^{2} \sigma_{s}^{2} \sigma_{n}^{2}\left(\sigma_{s}^{2}+\sigma_{n}^{2}\right)^{2}\right. \\
\left.+\left(32 L_{p}-28\right) \sigma_{s}^{4} \sigma_{n}^{4}\right]-8 \frac{\sigma_{s}^{4}}{L_{p}^{4}\left(\sigma_{s}^{2}+\sigma_{n}^{2}\right)^{6}}\left[\left(L_{p}-1\right)^{2} \sigma_{s}^{4} \sigma_{n}^{2}\left(\sigma_{s}^{2}+\sigma_{n}^{2}\right)\right. \\
\left.+\left(L_{p}-1\right) \sigma_{s}^{4} \sigma_{n}^{2}\left(\sigma_{s}^{2}+2 \sigma_{n}^{2}\right)+\left(L_{p}+1\right) \sigma_{s}^{2} \sigma_{n}^{2}\left(\sigma_{s}^{2}+\sigma_{n}^{2}\right)^{2}\right] \text { using the FIP preamble. } \\
\frac{1}{L_{p}^{4}\left(\sigma_{s}^{2}+\sigma_{n}^{2}\right)^{4}}\left[\left(L_{p}-1\right)^{2} \sigma_{s}^{4}\left(2 \sigma_{s}^{4}+8 \sigma_{s}^{2} \sigma_{n}^{2}+4 \sigma_{n}^{4}\right)+4\left(L_{p}-1\right)\left[3 \sigma_{s}^{4}\left(\sigma_{s}^{2} \sigma_{n}^{2}+\sigma_{n}^{4}\right)+\right.\right. \\
\left.\left.\left(2 \sigma_{s}^{2} \sigma_{n}^{2}+\sigma_{n}^{4}\right)^{2}\right]+8\left(\sigma_{s}^{6} \sigma_{n}^{2}+\sigma_{s}^{4} \sigma_{n}^{4}+\sigma_{s}^{2} \sigma_{n}^{6}\right)\right]+\frac{\sigma_{s}^{8}}{L_{p}^{4}\left(\sigma_{s}^{2}+\sigma_{n}^{2}\right)^{8}}\left[16 L_{p}^{2} \sigma_{s}^{2} \sigma_{n}^{2}\left(\sigma_{s}^{2}+\sigma_{n}^{2}\right)^{2}+\right. \\
\left.\left(32 L_{p}-28\right) \sigma_{s}^{4} \sigma_{n}^{4}\right]-8 \frac{\sigma_{s}^{4}}{L_{p}^{4}\left(\sigma_{s}^{2}+\sigma_{n}^{2}\right)^{6}}\left[\left(L_{p}-1\right)^{2} \sigma_{s}^{4} \sigma_{n}^{2}\left(\sigma_{s}^{2}+\sigma_{n}^{2}\right)+\right. \\
\left.\left(L_{p}-1\right) \sigma_{s}^{4} \sigma_{n}^{2}\left(\sigma_{s}^{2}+2 \sigma_{n}^{2}\right)+\left(L_{p}+1\right) \sigma_{s}^{2} \sigma_{n}^{2}\left(\sigma_{s}^{2}+\sigma_{n}^{2}\right)^{2}\right] \text { using SC's preamble. }
\end{array}\right.
$$


When $L_{p}$ is large, $\operatorname{Var}\left(M_{d}\right)$ can be approximated as

$$
\operatorname{Var}\left(M_{d}\right) \approx\left\{\begin{array}{l}
\frac{1}{L_{p}^{4}\left(\sigma_{s}^{2}+\sigma_{n}^{2}\right)^{4}}\left[4\left(L_{p}-1\right)^{2}\left(3 \sigma_{s}^{6} \sigma_{n}^{2}+\sigma_{s}^{4} \sigma_{n}^{4}\right)\right] \\
+\frac{\sigma_{s}^{8}}{L_{p}^{4}\left(\sigma_{s}^{2}+\sigma_{n}^{2}\right)^{8}}\left[16 L_{p}^{2} \sigma_{s}^{2} \sigma_{n}^{2}\left(\sigma_{s}^{2}+\sigma_{n}^{2}\right)^{2}\right] \\
-8 \frac{\sigma_{s}^{4}}{L_{p}^{4}\left(\sigma_{s}^{2}+\sigma_{n}^{2}\right)^{6}}\left[\left(L_{p}-1\right)^{2} \sigma_{s}^{4} \sigma_{n}^{2}\left(\sigma_{s}^{2}+\sigma_{n}^{2}\right)\right] \\
\text { using the FIP preamble. } \\
\frac{1}{L_{p}^{4}\left(\sigma_{s}^{2}+\sigma_{n}^{2}\right)^{4}}\left[\left(L_{p}-1\right)^{2} \sigma_{s}^{4}\left(2 \sigma_{s}^{4}+8 \sigma_{s}^{2} \sigma_{n}^{2}+4 \sigma_{n}^{4}\right)\right] \\
+\frac{\sigma_{s}^{8}}{L_{p}^{4}\left(\sigma_{s}^{2}+\sigma_{n}^{2}\right)^{8}}\left[16 L_{p}^{2} \sigma_{s}^{2} \sigma_{n}^{2}\left(\sigma_{s}^{2}+\sigma_{n}^{2}\right)^{2}\right] \\
-8 \frac{\sigma_{s}^{4}}{L_{p}^{4}\left(\sigma_{s}^{2}+\sigma_{n}^{2}\right)^{6}}\left[\left(L_{p}-1\right)^{2} \sigma_{s}^{4} \sigma_{n}^{2}\left(\sigma_{s}^{2}+\sigma_{n}^{2}\right)\right] \\
\text { using SC's preamble. }
\end{array}\right.
$$

(4.19-4.21) show that the FIP doubles the mean of $M_{d}$ (and decreases the variance of $M_{d}$ when SNR $>3 \mathrm{~dB}$ ). Since $M_{d}$ is approximated by a Gaussian RV, using the FIP decreases the probability of the start point shifting to the right side. Fig. 4.2 shows the comparison of the simulation and theoretic $M_{d}$ 's mean and variance using SC and proposed preambles in AWGN channels, where $L_{p}=512, N=1024$ and $\sigma_{s}^{2}=1 / N$. 100,000 simulation runs are done for each SNR.

Fig. 4.3 shows the timing metrics of different methods under a noise free and no distortion condition. The curve labeled "Proposed method" in Fig. 4.3 uses the AWGN preamble structure and the curve labeled "Without CP and postfix" uses as first training symbol only two identical parts. Park and Ren's methods are described in [9] and [10] respectively. From Fig. 4.3, we see the timing metric of the proposed method does not have the plateau of SC's method and the sub peaks of Minn's method. Its peak is quite sharp. 


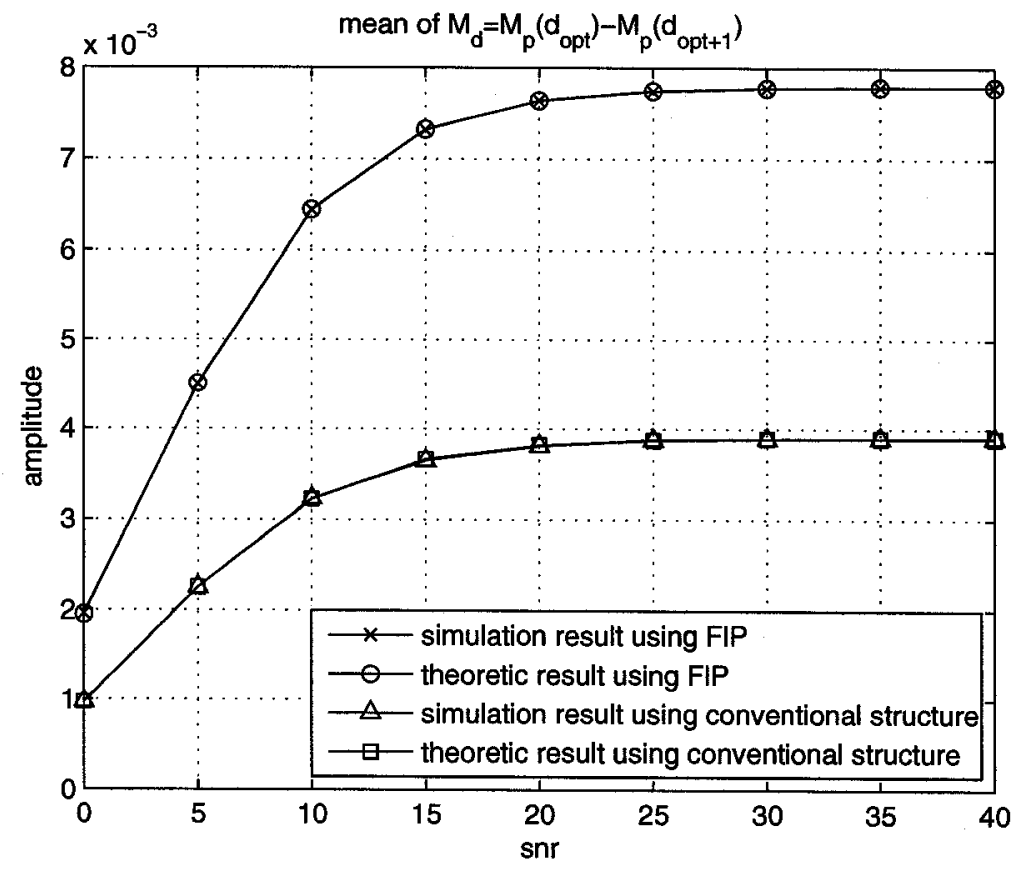

(a) Mean of $M_{d}$

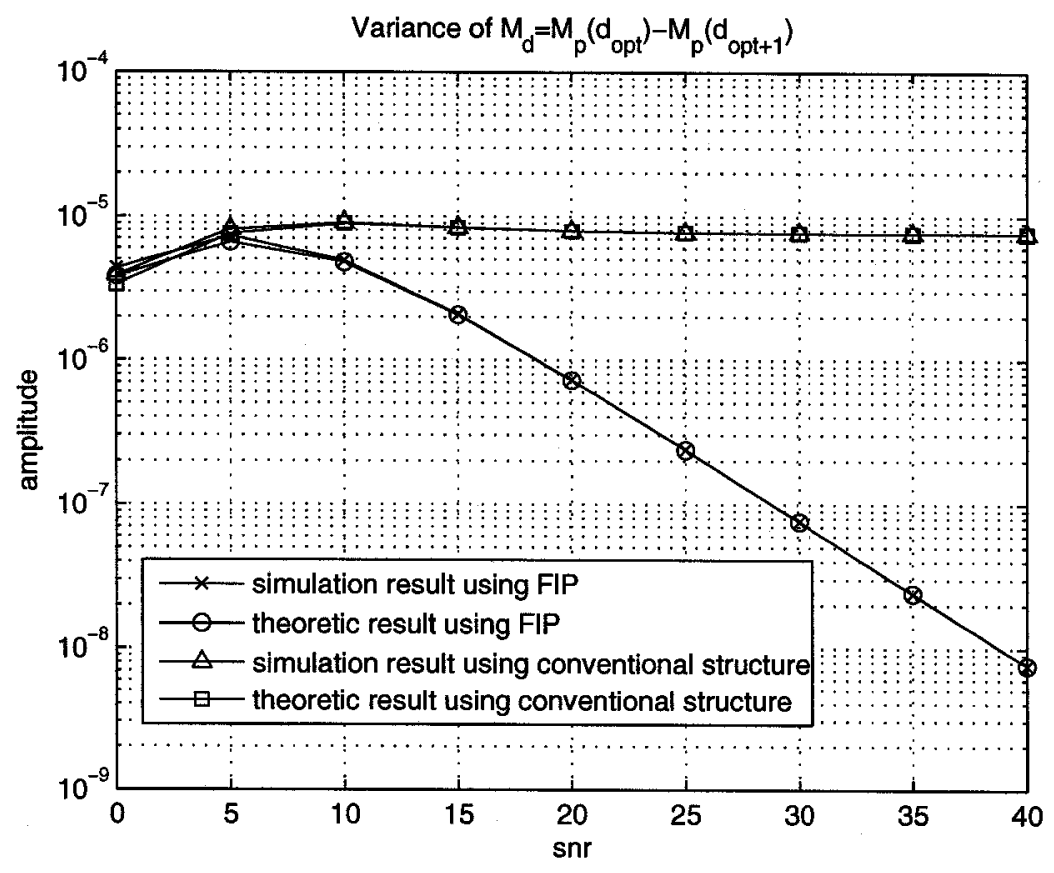

(b) Variance of $M_{d}$

Fig. 4.2 Comparison of simulated and theoretical $M_{d}$ statistic using SC and proposed preambles 


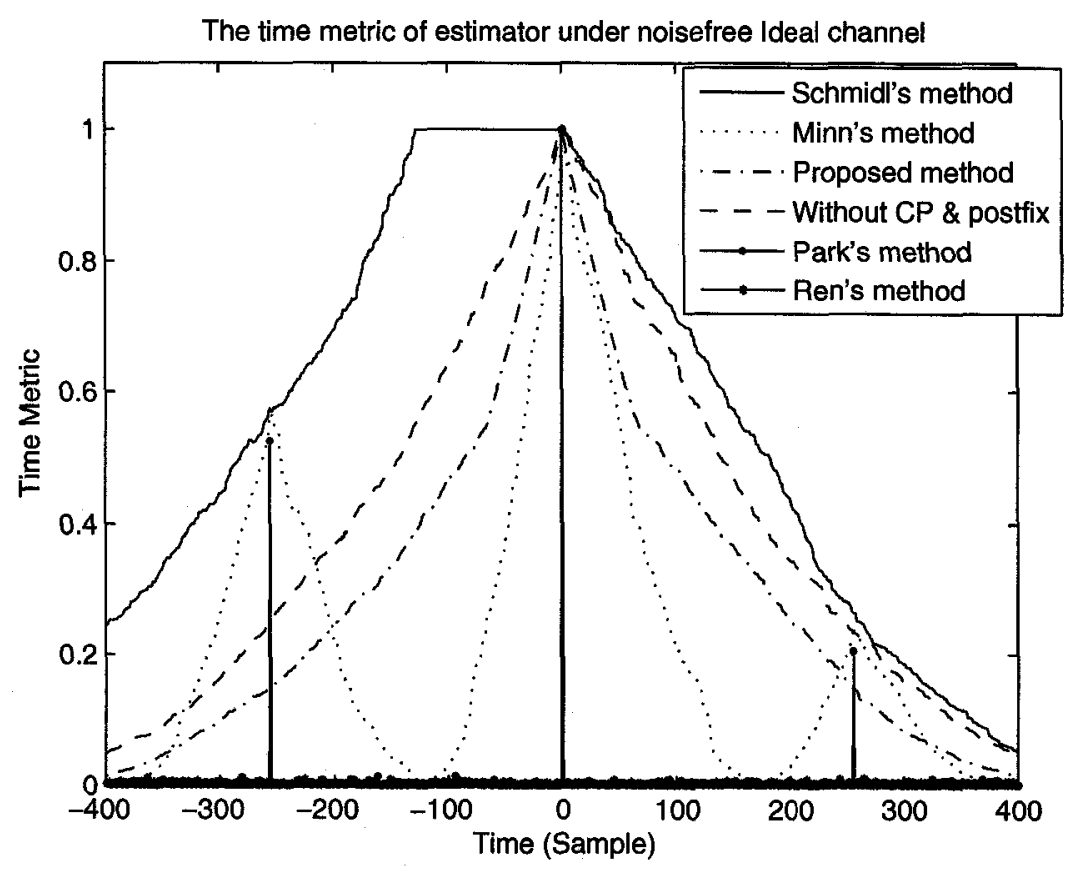

Fig. 4.3 Timing metric in a noise free ideal channel.

\subsection{Proposed synchronization method for MIMO-OFDM systems}

From section 2.4, a MIMO-OFDM system uses spatial diversity, spatial multiplexing, or combining both to achieve better performance and/or capacity gain under frequency selective fading channels. It also needs reliable synchronization methods. In this section, we are going to extend our proposed method from OFDM systems to MIMO-OFDM systems. SC's method can be extended by generalization. In section 4.2.1, we analyze the requirements of MIMO-OFDM preambles and give a preamble structure. In section 4.2.2, we describe the proposed MIMO-OFDM synchronization method. 


\subsubsection{Preamble structures for MIMO-OFDM systems}

If we use only one transmitter antenna, we can use the preamble discussed in section 4.1.1. If we use more than one transmitter antennas, the received signal would be a combination of the transmitted signals passed through the channel plus noise.

Now, let us analyze the optimum preamble in a multipath fading channel. Assume we use $Q$ transmitter antennas and $L$ receiver antennas. Let $h_{q l, m}$ be the channel impulse response at the lag $m$ from the $q^{\text {th }}$ transmitter antenna to the $l^{\text {th }}$ receiver antenna. The first training symbol contains two identical parts (denoted as $\left[\boldsymbol{a}_{\boldsymbol{q}, \mathbf{0}}, \boldsymbol{a}_{\boldsymbol{q}, \mathbf{0}}\right]$ for the $q^{\text {th }}$ transmitter). Let $\boldsymbol{a}_{\boldsymbol{q}, \boldsymbol{k}}$ denote the circular right shift $k$ samples version of $\boldsymbol{a}_{\boldsymbol{q}, \mathbf{0}}$. From (2.53), the received signal of the $l^{\text {th }}$ receiver antenna corresponding to the first symbol without noise is composed of two parts:

$$
\begin{aligned}
\boldsymbol{r}_{l, \mathbf{I}} & =\sum_{q=1}^{Q} \sum_{m=0}^{M-1} h_{q l, m} \boldsymbol{a}_{\boldsymbol{q}, \boldsymbol{m}} \\
\boldsymbol{r}_{l, \mathbf{I I}} & =e^{j \phi}\left(\sum_{q=1}^{Q} \sum_{m=0}^{M-1} h_{q l, m} \boldsymbol{a}_{\boldsymbol{q}, \boldsymbol{m}}\right)
\end{aligned}
$$

where $\phi$ is the phase difference between the two identical parts. The correlation function of the two parts for the $l^{\text {th }}$ receive antenna $P_{l}(d)$ defined as ${ }^{1}$ :

$$
P_{l}(d)=\sum_{m=0}^{N / 2-1} r_{l, d+m}^{*} r_{l, d+m+N / 2}
$$

where $r_{l, k}$ is given by (2.53). Let $d_{\text {opt }}$ be the exact start point of the symbol excluding prefix and postfix. $P_{l}\left(d_{\text {opt }}\right)$ can be expressed as the scalar product of the two vectors

\footnotetext{
${ }^{1}$ MIMO version of (4.1).
} 
$r_{l, \mathrm{I}}$ and $r_{l, \mathrm{II}}$ :

$$
\begin{aligned}
P_{l}\left(d_{o p t}\right) & =\boldsymbol{r}_{l, \mathbf{I}}^{\dagger} \boldsymbol{r}_{l, \mathbf{I I}} \\
& =e^{j \phi}\left(\sum_{q=1}^{Q} \sum_{m=0}^{M-1}\left|h_{q l, m}\right|^{2} \boldsymbol{a}_{\boldsymbol{q}, \boldsymbol{m}}^{\dagger} \boldsymbol{a}_{\boldsymbol{q}, \boldsymbol{m}}+\sum_{q=1}^{Q} \sum_{p=1}^{Q} \sum_{m=0}^{M-1} \sum_{n=0, n \neq m}^{M-1} h_{q l, m}^{*} h_{p l, n} \boldsymbol{a}_{\boldsymbol{q}, \boldsymbol{m}}^{\dagger} \boldsymbol{a}_{\boldsymbol{p}, \boldsymbol{n}}\right)
\end{aligned}
$$

where $\nmid$ is Hermitian conjugation of a matrix or a vector.

In (4.24), the term $\sum_{q=1}^{Q} \sum_{m=0}^{M-1}\left|h_{q l, m}\right|^{2} \boldsymbol{a}_{\boldsymbol{q}, \boldsymbol{m}}^{\dagger} \boldsymbol{a}_{\boldsymbol{q}, \boldsymbol{m}}$ always gives a positive contribution, but the second term $\sum_{q=1}^{Q} \sum_{p=1}^{Q} \sum_{m=0}^{M-1} \sum_{\substack{M-1 \\ n=0, n \neq m}}^{M-1} h_{q l}^{*} h^{*}{ }_{q, m} h_{p l, n} a_{q, \boldsymbol{m}}^{\dagger} \boldsymbol{a}_{\boldsymbol{p}, \boldsymbol{n}}$ might give a negative or positive contribution depending on the channel coefficients and training symbols. To eliminate the second term effect, we might set it to be zero, i.e, the training sequences of different antennas should be orthogonal and shift-orthogonal and each training sequence of different antennas should have a perfect autocorrelation function. Since we assume the channel tap length is no longer than the guard interval (CP length), we use Chu sequences and each circular shift $G$ version of Chu sequences to be the half part of the first training symbol for each transmitter antenna. Let the first training symbol of the first transmitter antenna be $\left[\boldsymbol{a}_{\mathbf{1 , 0},}, \boldsymbol{a}_{\mathbf{1 , 0}}\right]$, where $\boldsymbol{a}_{\mathbf{1 , 0}}$ is a Chu sequence. And let the first training symbol of the $q^{\text {th }}$ transmitter antenna be $\left[a_{1,(q-1) G}, a_{1,(q-1) G}\right]$, where $\boldsymbol{a}_{1,(q-1) G}$ is circular right shift $(q-1) G$ samples version of $\boldsymbol{a}_{\mathbf{1 , 0}}$. If the number of transmitter antennas $Q$ is greater than $\left\lfloor\frac{N}{2 G}\right\rfloor$, we cannot achieve the shift-orthogonality.

For the proposed method, to make the training sequences of different antennas orthogonal and shift-orthogonal with each other, we have to modify the first training symbol structure for ISI or fading channels. In addition to the two identical parts $\left[\boldsymbol{a}_{\boldsymbol{q}, \mathbf{0}}, \boldsymbol{a}_{\boldsymbol{q}, \mathbf{0}}\right]=\left[\boldsymbol{a}_{1,(\boldsymbol{q}-1) G}, \boldsymbol{a}_{1,(q-1) G}\right]$ which are Chu sequences of length $L_{p}=N / 2$, we 
add a CP and a Flipped Postfix (FIP) of lengths $G / 2$ to the first symbol of each transmitter antenna (see Fig. $4.1 \mathrm{~d}$ ).

Let us denote the second training symbol (excluding CP) for the $q^{\text {th }}$ transmitter antenna as $\boldsymbol{b}_{\boldsymbol{q}, \mathbf{0}}$. In OFDM systems, the differences of two training symbols on the even subcarriers can be used to estimate the integer part CFO. More specifically, using an approach similar to SC'method, the CFO integer part is obtained using the ratio sequence $c_{k}$ defined as

$$
c_{k}=X(q, 1, k) / X(q, 2, k) \quad k=0,2, \ldots, N-2
$$

where $\{X(q, 1, k)\}$ is the FFT of $\left[\boldsymbol{a}_{1,(q-1) G}, \boldsymbol{a}_{1,(q-1) G}\right]$ and $\{X(q, 2, k)\}$ is the FFT of $\boldsymbol{b}_{\boldsymbol{q}, \mathbf{0}}$, and $k$ is the index of the subcarrier. For MIMO systems with multiple transmitter antennas, the $\left\{c_{k}\right\}$ must be identical for all transmitter antennas in order to get a better estimation for the CFO integer part. This can be achieved by letting $\boldsymbol{b}_{\boldsymbol{q}, \mathbf{0}}=$ $\boldsymbol{b}_{1,(q-1) G}$, where $\boldsymbol{b}_{1,(q-1) G}$ is the circular shift $(q-1) G$ samples version of $\boldsymbol{b}_{1,0}$ and $\boldsymbol{b}_{\mathbf{1}, \mathbf{0}}$ is chosen as a Chu sequence. Since the second symbol shifts by the same number of samples as the first symbol for each transmitter antenna, by the circular time shifting property of $\mathrm{DFT}^{2}$, the sequence $\left\{c_{k}\right\}$ is the same for all transmitter antennas.

\subsubsection{Implementing the proposed synchronization method for MIMO-OFDM systems}

Similar as the proposed synchronization method for OFDM systems, the proposed synchronization method for MIMO-OFDM systems has four steps:

\footnotetext{
${ }^{2}$ From [26], the circular time shifting property of the DFT is: Let $g[n]$ be a length $N$ sequence and $G[k]$ be the $N$-point DFT of $g[n]$, then $e^{\frac{-j 2 \pi k n_{0}}{N}} G[k]$ is the $N$-point DFT of $g\left[\left(n-n_{0}\right)_{N}\right]$. where $\left(n-n_{0}\right)_{N}$ is $\bmod \left(\left(n-n_{0}\right) / N\right)$.
} 
- Step 1) Coarse Time Synchronization

First, we do the coarse time synchronization for each receiver antennas. Let the $l^{\text {th }}$ receiver antenna timing metric be $M_{l}$ :

$$
M_{l}(d)=\frac{\left|P_{l}(d)\right|^{2}}{R_{l}^{2}(d)}
$$

where $P_{l}(d)$ is given by (4.23) and

$$
R_{l}(d)=\frac{1}{2} \sum_{m=0}^{N-1}\left|r_{l, d+m}\right|^{2}
$$

where $r_{l, k}$ is the $k^{\text {th }}$ complex sample taken at the output of A/D of the $l^{\text {th }}$ receiver antenna given by $(2.53)$. Note that $R_{l}(d)$ corresponds to the so-called modified "R" following [7] (see (3.12)). The coarse time estimation of the $l^{\text {th }}$ receive antenna is

$$
\widehat{d}_{l, c o a r s e}=\arg \underbrace{\max }_{d}\left\{M_{l}(d)\right\}
$$

and the estimated SNR of the $l^{\text {th }}$ receiver antenna is [6]

$$
\widehat{S N R_{l}}=\frac{\sqrt{M_{l}\left(\widehat{d}_{l, \text { coarse }}\right)}}{1-\sqrt{M_{l}\left(\widehat{d}_{l, \text { coarse }}\right)}}
$$

The timing metric for MIMO-OFDM systems is defined:

$$
M_{M I M O}(d)=\sum_{l=1}^{L} w_{l} M_{l}(d)
$$

where $w_{l}$ is the weight of each receiver antenna timing metric. If $w_{l}=1$, the system implements equal gain combining. $w_{l}=\widehat{S N R_{l}}$ corresponds to maximum ratio combining. The coarse estimation of the start point is taken at the peak 
of the timing metric $M_{M I M O}(d)$ and it is given by

$$
\widehat{d}_{\text {coarse }}=\arg \underbrace{\max }_{d}\left\{M_{M I M O}(d)\right\}
$$

- Step 2) Estimating the Fraction Part of the CFO

To estimate the fraction part of the CFO, we define

$$
P_{M I M O}\left(\widehat{d}_{c o a r s e}\right)=\sum_{l=1}^{L} w_{l} P_{l}\left(\widehat{d}_{c o a r s e}\right)
$$

where $w_{l}$ is the weight of each receiver antenna defined as in step 1. Similar to the OFDM system, the estimated fraction part of the CFO is

$$
\widehat{\gamma_{f}}=\frac{\angle P_{M I M O}\left(\widehat{d}_{\text {coarse }}\right)}{\pi}
$$

In this step, the frequency offset estimation is up to \pm 1 subcarrier spacing. To remove the fraction part of the CFO, we should multiply the received sample sequences with $e^{-j 2 \pi \widehat{\gamma f} k / N}$.

- Step 3) Estimating the Integer Part of the CFO

The actual frequency offset is

$$
\gamma=\gamma_{f}+2 g
$$

where $g$ is an integer. To estimate the integer part of the CFO $g$, we have to use the second training symbol. After the fraction CFO correction (by multiplying the samples by $e^{-j 2 \pi \widehat{\gamma f}_{f} k / N}$ ), let FFT of the $l^{\text {th }}$ receiver antenna's first symbol be $x_{l, 1, k}$ and the FFT of the $l^{t h}$ receiver antenna's second symbol be $x_{l, 2, k}$. Let $\left\{c_{k}\right\}$ 
be the ratio sequence on the even frequencies of the two training symbols.

$$
c_{k}=X(q, 1, k) / X(q, 2, k) \quad k=0,2, \cdots, N-2
$$

where $X(q, 1, k)$ and $X(q, 2, k)$ are frequency training sequences of the first and second training symbol from the $q^{\text {th }}$ transmitter antenna respectively and $k$ is index of the subcarrier. As discussed in section 4.2.1, all transmitter antennas have same ratio sequences $\left\{c_{k}\right\}$. Let

$$
G_{l}(g)=\frac{\left|\sum_{k \in \boldsymbol{X}} x_{l, 2, k+2 g}^{*} c_{k}^{*} x_{l, 1, k+2 g}\right|^{2}}{2\left(\sum_{k \in \boldsymbol{X}}\left|x_{l, 1, k}\right|^{2}\right)^{2}}
$$

where $\boldsymbol{X}$ is the set of indices for the even frequency components given by $\boldsymbol{X}=$ $\{0,2, \cdots, N-2\}$. The number of the even positions shifted can be calculated by finding $\widehat{g}$ that maximizes

$$
G_{M I M O}(g)=\sum_{l=1}^{L} w_{l} G_{l}(g)
$$

where $w_{l}$ is the weight of each receiver antenna defined as in step 1 . The estimated integer part of the CFO is

$$
\widehat{g}=\arg \underbrace{\max }_{g}\left\{G_{M I M O}(g)\right\}
$$

The estimated CFO is

$$
\widehat{\gamma}=\widehat{\gamma_{f}}+2 \widehat{g}
$$

If we multiply the received sample sequences with $e^{-j 2 \pi \hat{\gamma} k / N}$, the CFO is removed. 


\section{Proposed synchronization method for OFDM and MIMO-OFDM}

- Step 4) Fine Time Synchronization

First, we have to remove the CFO effect by multiplying the received sample sequences of each receiver antenna with $e^{-j 2 \pi \hat{\gamma k} / N}$. The $k^{\text {th }}$ carrier frequency corrected time domain sample at the $l^{\text {th }}$ receiver antenna is

$$
r_{l, k}^{c}=r_{l, k} e^{-j 2 \pi \hat{\gamma} k / N}
$$

Secondly, we calculate the cross-correlation of the carrier frequency corrected time domain sequences $\left\{r_{l, k}^{c}\right\}$ and the transmitted preamble sequences of each transmitter antenna and average over all transmitter antennas to obtain:

$$
\begin{aligned}
& \psi_{l, n}^{\prime}=\sum_{q=1}^{Q}\left|\sum_{k=0}^{N-1} b_{q, k}^{*} r_{l, n+k}^{c}\right| \\
& n \in\left[-G / 2+\widehat{d}_{\text {coarse }}+N+1.5 G, \widehat{d}_{\text {coarse }}+N+1.5 G+G / 2\right]
\end{aligned}
$$

where $\boldsymbol{b}_{\boldsymbol{q}, \mathbf{0}}=\left[b_{q, 0}, \ldots, b_{q, N-1}\right]$ is the second time training sequence of the $q^{\text {th }}$ transmitter antenna excluding CP. Since the training sequences for different transmitter antennas are orthogonal, only one cross-correlation is needed per receiver antenna. Using the first transmitter antenna sequences $\left\{b_{1, k}\right\}$ to do the correlation, we have

$$
\begin{aligned}
& \psi_{l, n}=\left|\sum_{k=0}^{N-1} b_{1, k}^{*} r_{l, n+k}^{c}\right| \\
& n \in\left[-G / 2+\widehat{d}_{\text {coarse }}+N+1.5 G, \widehat{d}_{\text {coorse }}+N+1.5 G+G / 2\right]
\end{aligned}
$$

For each receiver antenna, we search the first point $\widehat{n}$ in $\left[-G / 2+\widehat{d}_{\text {coarse }}+N+\right.$ $\left.1.5 G, \widehat{d}_{c o a r s e}+N+1.5 G+G / 2\right]$ (from smallest to greatest) whose $\psi_{l, \widehat{n}}$ is greater 
than a threshold $\eta_{l, \text { Rayleigh }}$. Then we obtain fine time synchronization using $\widehat{d}_{l, \text { fine }}=\widehat{n}-N-1.5 G$ and

$$
\widehat{d}_{\text {fine }}=\left\lfloor\frac{1}{L} \sum_{l=1}^{L} \widehat{d}_{l, f i n e}\right\rfloor
$$

The choice of threshold for each receiver antenna is obtained similarly to the threshold for SISO OFDM systems. Assuming that the first tap power would be greater than $-14 \mathrm{~dB}$ (or equivalently $\left|h_{1 l, 0}\right|>0.2$ ), it can be shown that a good threshold is $\eta_{l, \text { Rayleigh }}=0.5 E\left[\psi_{l, d_{o p t}}\right] \approx 0.2 R_{l}\left(\widehat{d}_{C}\right) \sqrt{M_{l}\left(\widehat{d}_{C}\right)}$. Since we can estimate SNR from the peak value of the timing metric, we can also set up a look up table depending on the estimated SNR for setting the threshold. For example, in the simulations of this thesis we set

$$
\eta_{l, \text { Rayleigh }}= \begin{cases}0.2 R_{l}\left(\widehat{d}_{C}\right) \sqrt{M_{l}\left(\widehat{d}_{C}\right)} & \text { if } \widehat{\mathrm{SNR}_{l}}>5 \mathrm{~dB} \\ 0.3 R_{l}\left(\widehat{d}_{C}\right) \sqrt{M_{l}\left(\widehat{d}_{C}\right)} & \text { if } \widehat{\mathrm{SNR}_{l}} \leq 5 \mathrm{~dB}\end{cases}
$$




\section{Chapter 5}

\section{Simulation results}

The performance of the proposed method is evaluated by computer simulations in terms of the mean and variance of the timing estimator and CFO estimator under AWGN, ISI or fading channels.

\subsection{Simulation parameters}

For all the simulations, the system uses 1024 subcarriers and 1024 point IFFT/FFT. The length of $\mathrm{CP}$ is $G=102$ samples. The $\mathrm{CFO}$ is 12.4 subcarrier spacing. We use QPSK modulation and 10,000 simulations are run for each SNR. We did not consider the windowing in the simulations. All simulations consider a continuous communication scenario since its self interference is worse than the one in burst communication. For a fair comparison, the SNR is defined as the average signal power and the average noise power ratio on one OFDM symbol excluding the postfix and prefix.

Recall the OFDM system model in section 2.5.1 and MIMO-OFDM systems model in section 2.5.2. For the simplicity of computer simulations, we set $n_{\epsilon}=0$ and $\theta_{0}=0$ in (2.49) and (2.53). 
For OFDM systems, we compare the SC's, Minn's and proposed method in terms of the mean and variance of the timing estimator and CFO estimator under AWGN, ISI or Rayleigh fading channels. For the ISI channels, we use the ISI channel models described in [7] and [6]. We simulate two types of ISI channels. Both ISI channels have 16 paths with an equal path spacing of 4 samples. The delays $\tau_{n}$ are $0,4,8, \cdots, 60$ samples and path gains are given by

$$
h_{n}=\frac{e^{-\tau_{n} / 60}}{\sqrt{\sum_{k=1}^{16} e^{-\tau_{k} / 30}}}, \quad n=1,2, \cdots, 16
$$

The phases of all paths are zeroes in the first ISI channel and uniformly distributed in $[0,2 \pi)$ in the second ISI channel.

For Rayleigh fading channels, we use the tapped delay line channel model described in section 2.1.4. From section 2.1.1, the tap weights $\left\{h_{n}(t)\right\}$ are zero mean complex Gaussian random processes and statistically independent. The channel power delay profile is modeled as an exponential power delay profile, truncated to 16 paths (see section 2.1.1). All simulated Rayleigh fading channels have 16 paths with delays $\tau_{n}$ of $0,4,8, \cdots, 60$ samples and mean power of each path obtained from (2.3) and given by

$$
E\left\{\left|h_{n}(t)\right|^{2}\right\}=\frac{e^{-\tau_{n} / 30}}{\sqrt{\sum_{k=1}^{16} e^{-\tau_{k} / 30}}}, \quad n=1,2, \cdots, 16 .
$$

Both time-invariant Rayleigh fading channels $\left(\left\{h_{n}(t)\right\}\right.$ does not change for all symbols and samples) and time-variant Rayleigh fading channels $\left(\left\{h_{n}(t)\right\}\right.$ changes each sample period depending on the maximum Doppler frequency) are simulated. We assume all taps have the same (Jake/Clarke) Doppler power spectrum given by (2.6). To generate the Doppler power spectrum given by (2.6), we can use the simulation method given by [27], but it is difficult to create multiple uncorrelated fading waveforms. Therefore, 
we use modified Jakes fading model provided by [28]. The carrier frequency of the system is $5 \mathrm{GH}_{\mathrm{z}}$. The total bandwidth of the system is $W=50 \mathrm{MH}_{\mathrm{z}}$ and the signal bandwidth is $W_{s}=45.47 \mathrm{MH}_{\mathrm{z}}$.

In all OFDM simulation figures, the label "modified $\mathrm{R}$ " in the symbol timing estimator curves means that $R_{f}(d)$ given by (3.12) is used instead of $R_{S C}(d), R_{M i n n}(d)$ or $R_{p}(d)$ in each timing metric. Note that Minn's [7] did not propose any method to estimate the integer part of the CFO hence Minn's method is not included in the figures presenting performance of estimators of the entire CFO (e.g. fraction plus integer part of $\mathrm{CFO})$.

For MIMO-OFDM systems, we only compare the SC'method with the proposed method. We use the same parameters as for OFDM systems. The power of the transmitter antennas is given by (2.52) for a fair comparison. All the timing metrics use $R_{l}(d)$ (given by (4.26)) instead of $R_{S C}(d)$ or $R_{p}(d)$.

For the CFO estimator, for OFDM systems the label "fine time" means using $\frac{\angle P_{p}\left(\hat{d}_{\text {fine }}\right)}{\pi}$ to estimate the fraction part of the CFO instead of $\frac{\left\langle P_{p}\left(\widehat{d}_{\text {coarse }}\right)\right.}{\pi}$ (see $\left.(4.8)\right)$.

\subsection{Simulation results in an AWGN channel}

Figs. 5.1, 5.2 and 5.3 present the simulation results in terms of the means and variances of the symbol timing and CFO estimators for SC's, Minn's and proposed methods in an AWGN channel.

Fig. 5.1 shows the performance of the symbol timing estimators for different methods in an AWGN channel. Fig. 5.1(a) shows that the mean value of SC's coarse timing estimator is quite far from the exact start point ("0 sample value") and on the left side of it due to the CP. For example, Fig. 5.1(a) shows for SC's method at $S N R=2 \mathrm{~dB}$, mean $=-50$. It means that the estimated start point has a mean which is 50 samples 
before the exact start point. The mean values of the other two estimators and SC's fine timing estimator are almost at the exact start point. Comparing all coarse timing estimators, the proposed coarse timing estimator has the smallest variance (see Fig. 5.1(b)). The variance of the proposed fine timing estimator is less than $10^{-4}$ when $S N R \geq-2 \mathrm{~dB}$ and the variance of the SC's fine timing estimator is less than $10^{-4}$ when $S N R \geq 2 \mathrm{~dB}$. Fig. 5.1 also shows that all modified methods have smaller variance than the original methods, which is expected since the variance of $R_{f}(d)$ is half of the variance of $R_{S C}(d), R_{M i n n}(d)$ or $R_{p}(d)$ from the law of large number.

Fig. 5.2 and Fig. 5.3 show the performance of the CFO estimators for different methods in an AWGN channel. The variance of the proposed CFO estimator is slightly smaller than the one of SC's CFO estimator using the coarse time point. Using the fine time point or the coarse time point to estimate CFO yields almost same performance. For Minn's CFO estimator, since its repeated part length is half of the SC's one, its mean is not accurate at low SNR and its variance is quite large. It has the worst performance among all three CFO estimators. The integer part of the CFO is estimated correctly for 10,000 runs by SC's and proposed methods for each simulated SNR.

From the discussion above, we conclude that the proposed method has the best performance in an AWGN channel. 


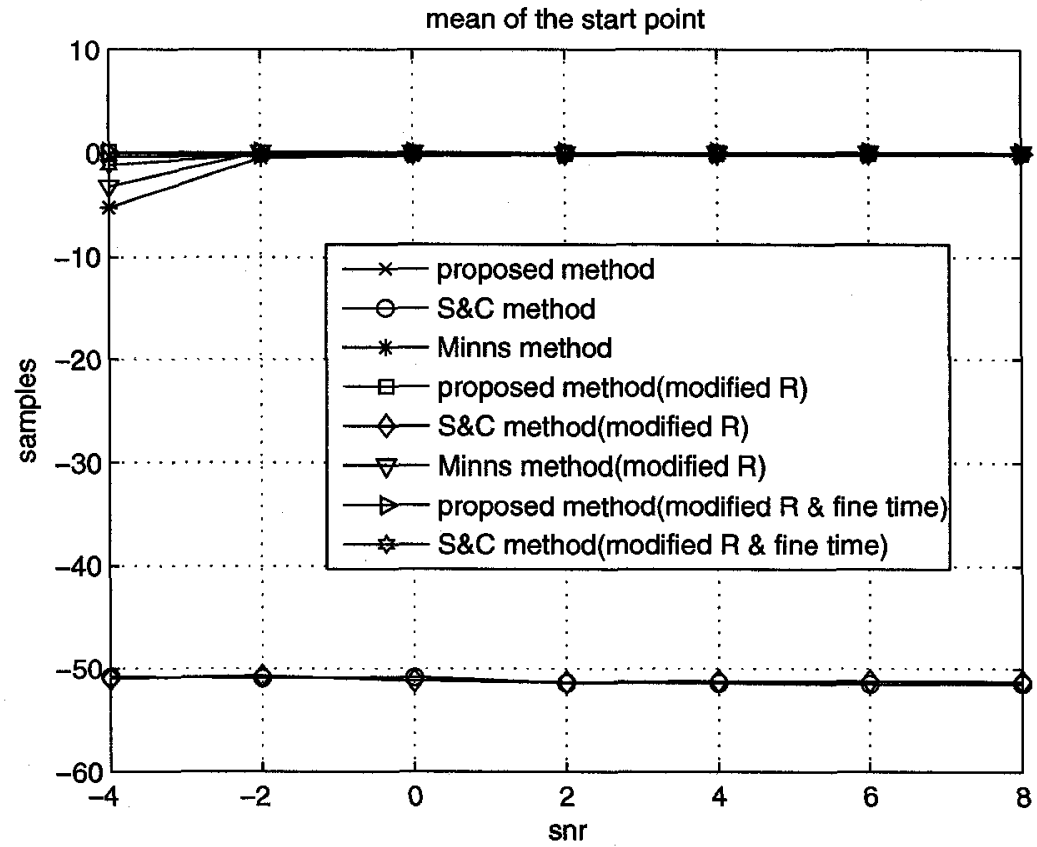

(a) Mean of the start point

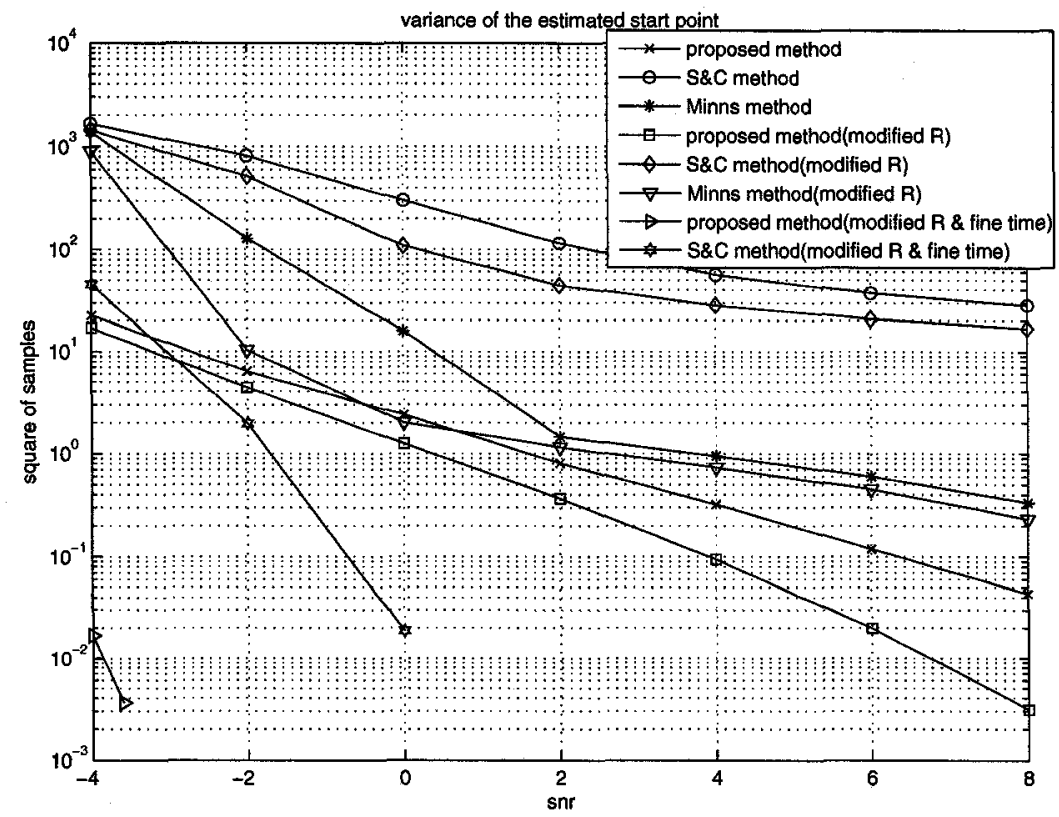

(b) Variance of the start point

Fig. 5.1 Performance of the timing estimator in an AWGN channel 


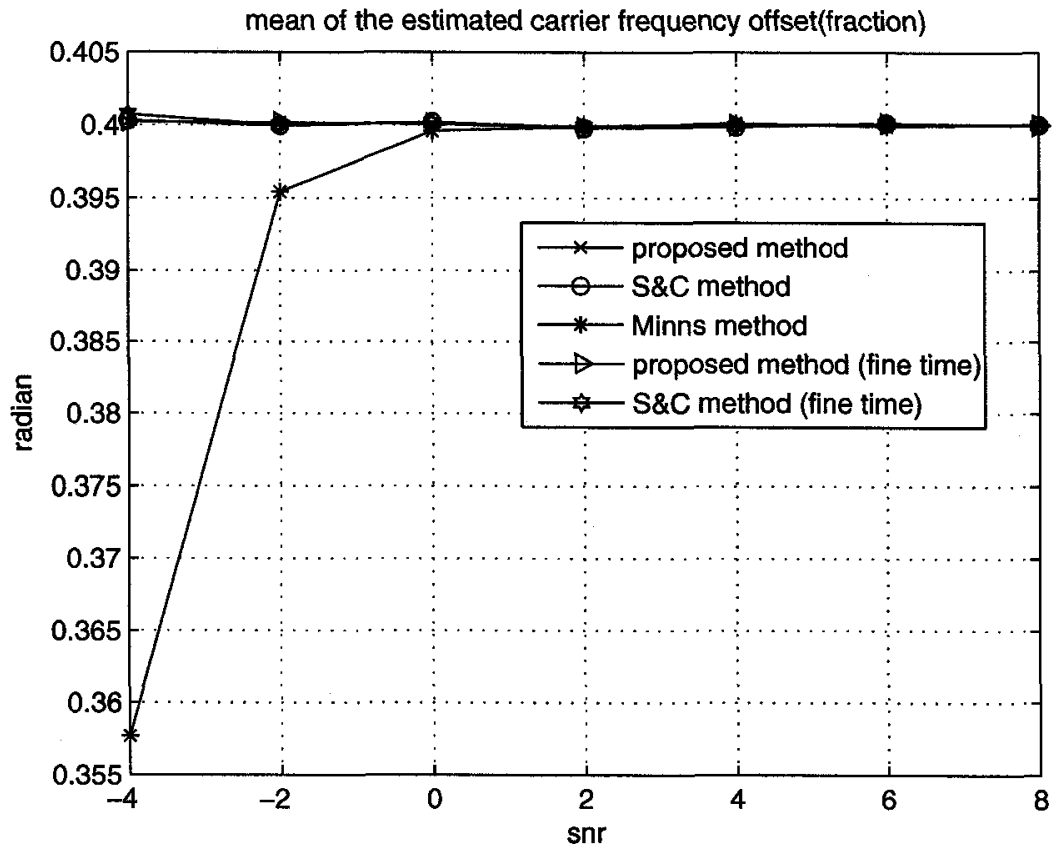

(a) Mean of the fraction part of the CFO

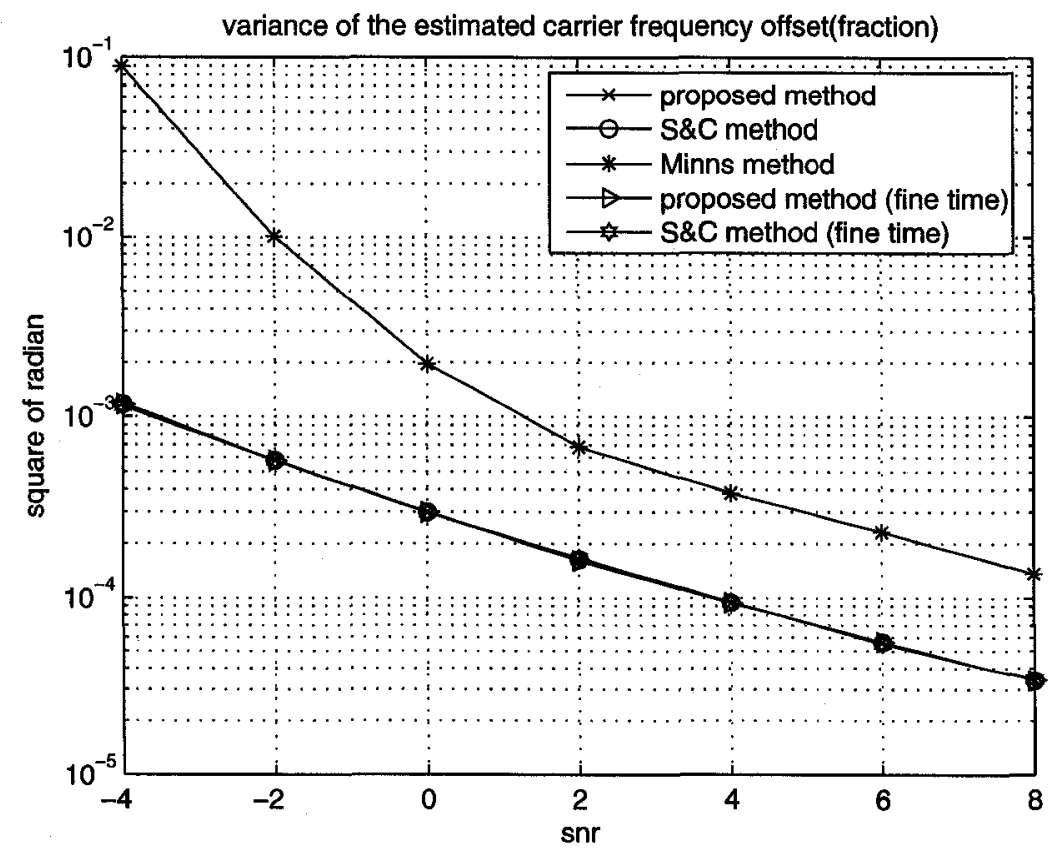

(b) Variance of the fraction part of the CFO

Fig. 5.2 Performance of the CFO (fraction part) estimator in an AWGN channel 


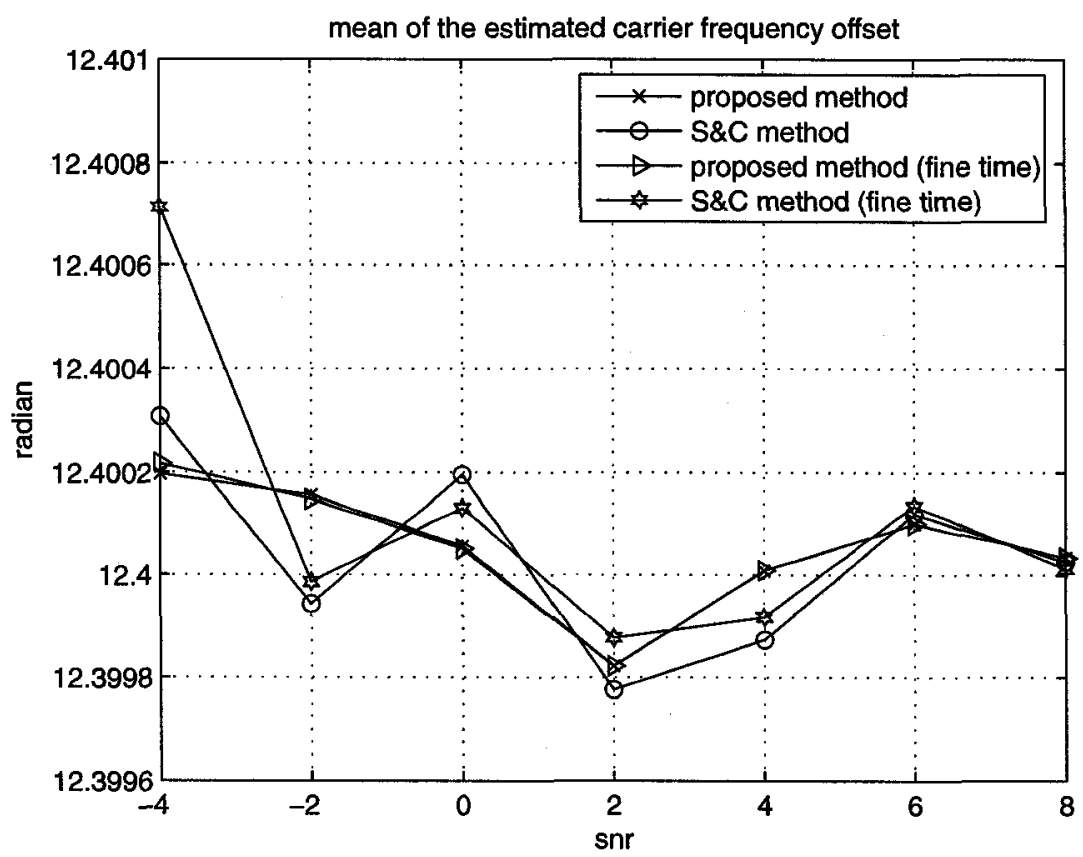

(a) Mean of the CFO

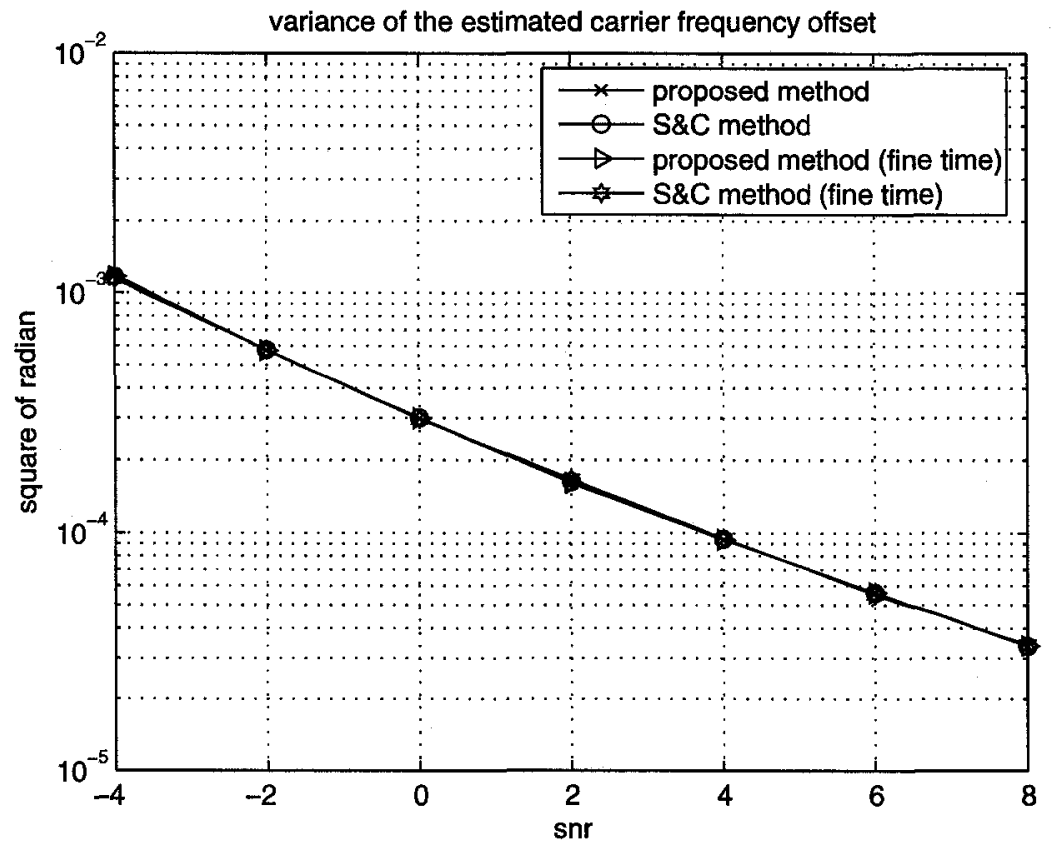

(b) Variance of the CFO

Fig. 5.3 Performance of the CFO estimator in an AWGN channel 


\subsection{Simulation results in the first ISI channel}

Figs. 5.4, 5.5 and 5.6 present the simulation results in terms of the means and variances of the symbol timing and CFO estimators for SC's, Minn's and proposed methods in the first ISI channel.

Fig. 5.4 shows the performance of the symbol timing estimators for different methods in the first ISI channel. Fig. 5.4(a) shows that the mean value of SC's coarse timing estimator shifts to the left side of the exact start point ('O' sample) due to the CP. The mean value of Minn's timing estimator shifts to the right side of the exact start point due to multipath effect. Note that Minn's results presented in Figs. 5.4, 5.5 and 5.6 were obtained by considering a training sequence (among all possible random sequences) that yielded performance similar to results presented in [7]. It was observed that this choice corresponded to almost the best training sequence in terms of performance of the timing estimator variance. If one uses Chu sequences instead then performance over the first ISI channel would be similar to the performance over the second ISI channel which will be presented in section 5.4. Fig. 5.4(a) shows that the mean values of the proposed coarse timing estimators (unmodified and modified " $R$ ") are quite close to the exact start point and always on the left side of it. As expected, mean values get closer to the exact start point as SNR increases. The mean values of the two fine timing estimators (SC and proposed) are almost at the exact start point. Since we pad $G / 2$ zeroes at the beginning of the training symbol, the variance of the proposed coarse timing estimator is the smallest one at high SNR and slightly larger than Minn's estimator at low SNR. But since the mean value of the proposed estimator is on the left side of the exact start point, most of the estimated start points would be on the left of it and since we use CP for data transmission after the training, the resulting ISI would be small. 


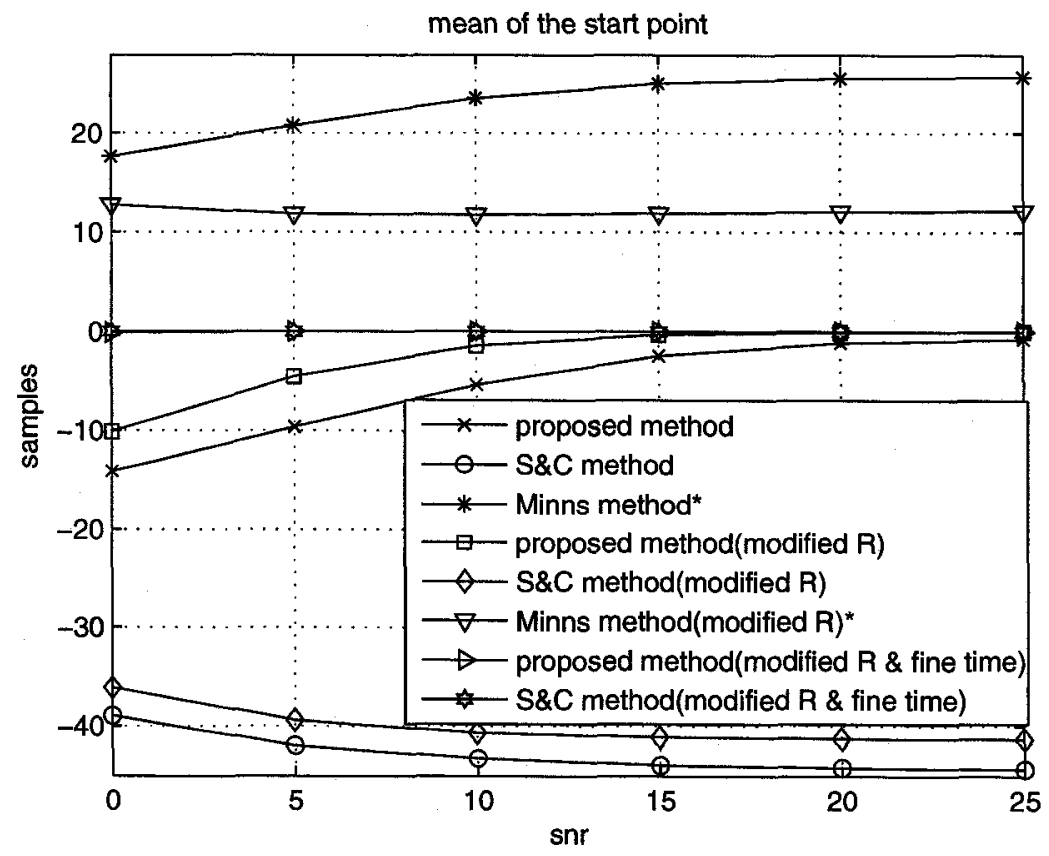

(a) Mean of the start point

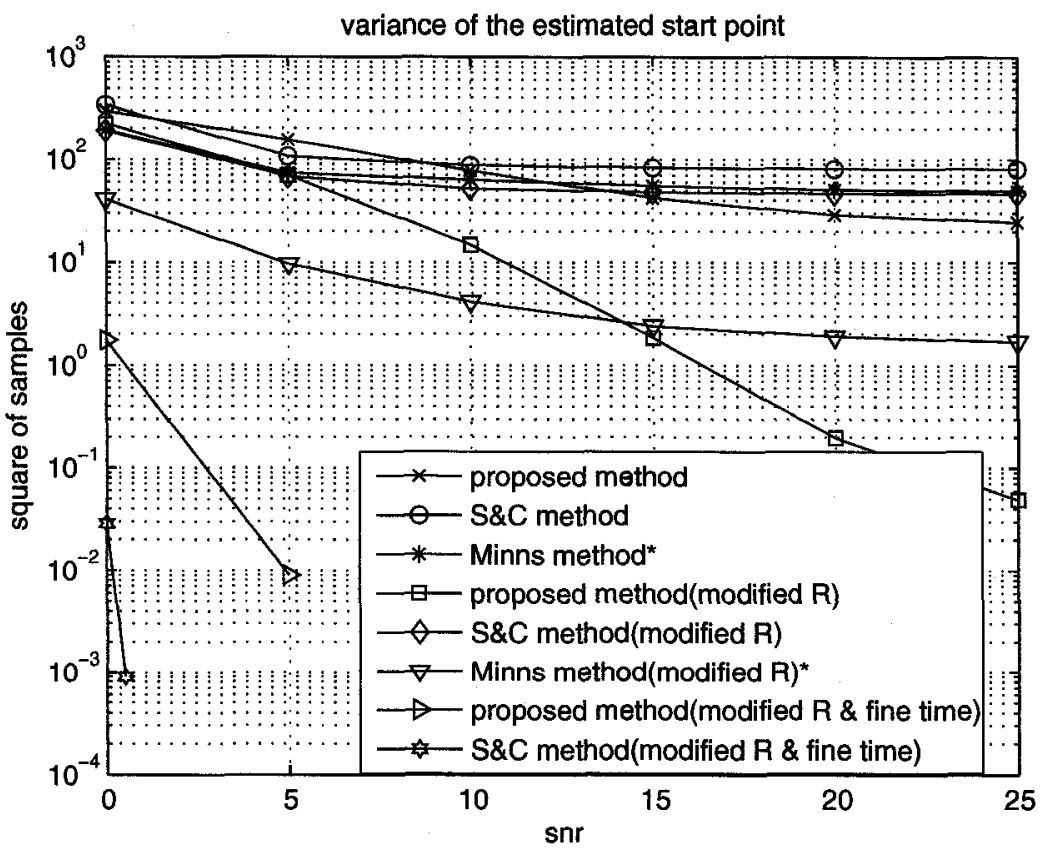

(b) Variance of the start point

Fig. 5.4 Performance of the timing estimator in the first ISI channel (*: uses training sequences similar to [7]) 
Fig. 5.4(b) shows that the proposed and SC's fine timing estimators have quite small variance. The variance of the proposed fine timing estimator is less than $10^{-4}$ when $S N R \geq 10 \mathrm{~dB}$ and the variance of the SC's fine timing estimator is less than $10^{-4}$ when $S N R \geq 5 \mathrm{~dB}$. Similar to AWGN results, Fig. 5.4(b) also shows that all modified methods have smaller variance than the original methods as expected.

Fig. 5.5 and Fig. 5.6 show the performance of the CFO estimators for different methods in the first ISI channel. The proposed CFO estimator has almost the same performance as SC's CFO estimator. Using the fine time point or the coarse time point to estimate CFO yields almost same performance. For Minn's CFO estimator, since its repeated part length is half of the SC's one, its mean is not accurate and its variance is quite large (see Fig. 5.5). It has the worst performance among all three CFO estimators in the first ISI channel, which was also observed in an AWGN channel. The integer part of the CFO is estimated correctly for 10,000 runs by SC's and proposed methods for each simulated SNR.

From the discussion above, we conclude that the SC's method (fine time) has the best performance in the first ISI channel. 


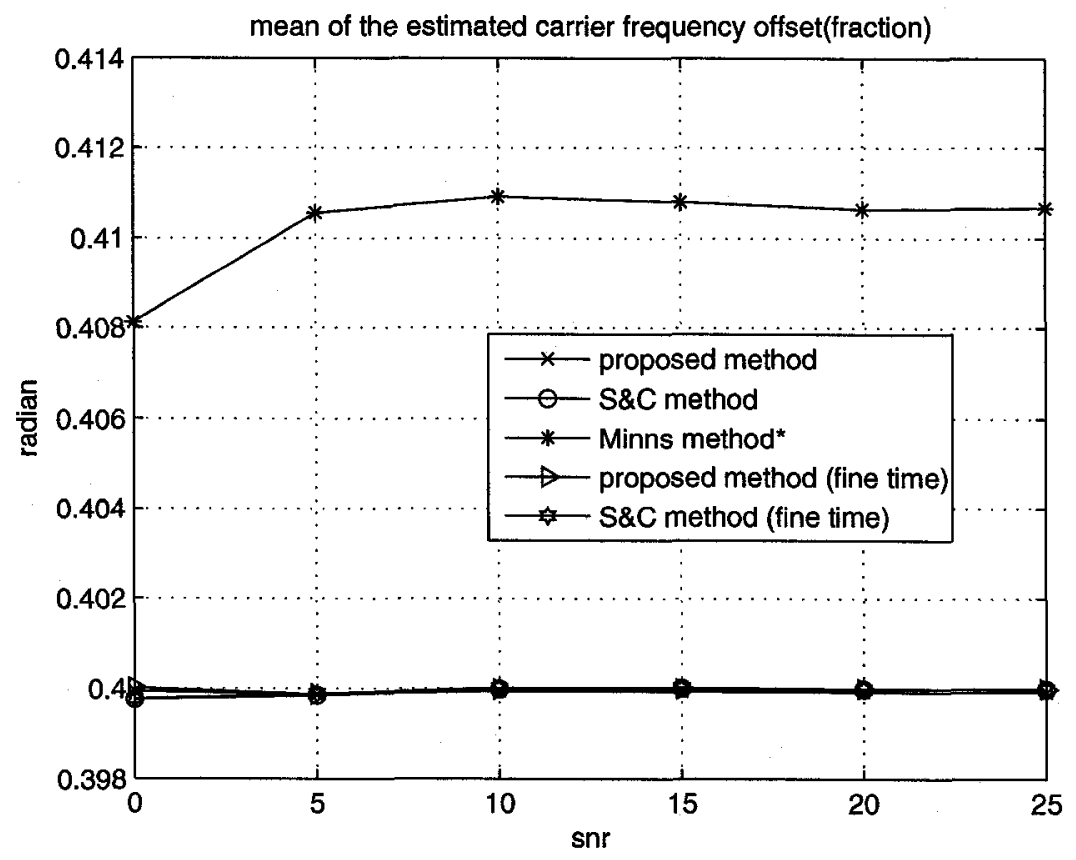

(a) Mean of the fraction part of the CFO

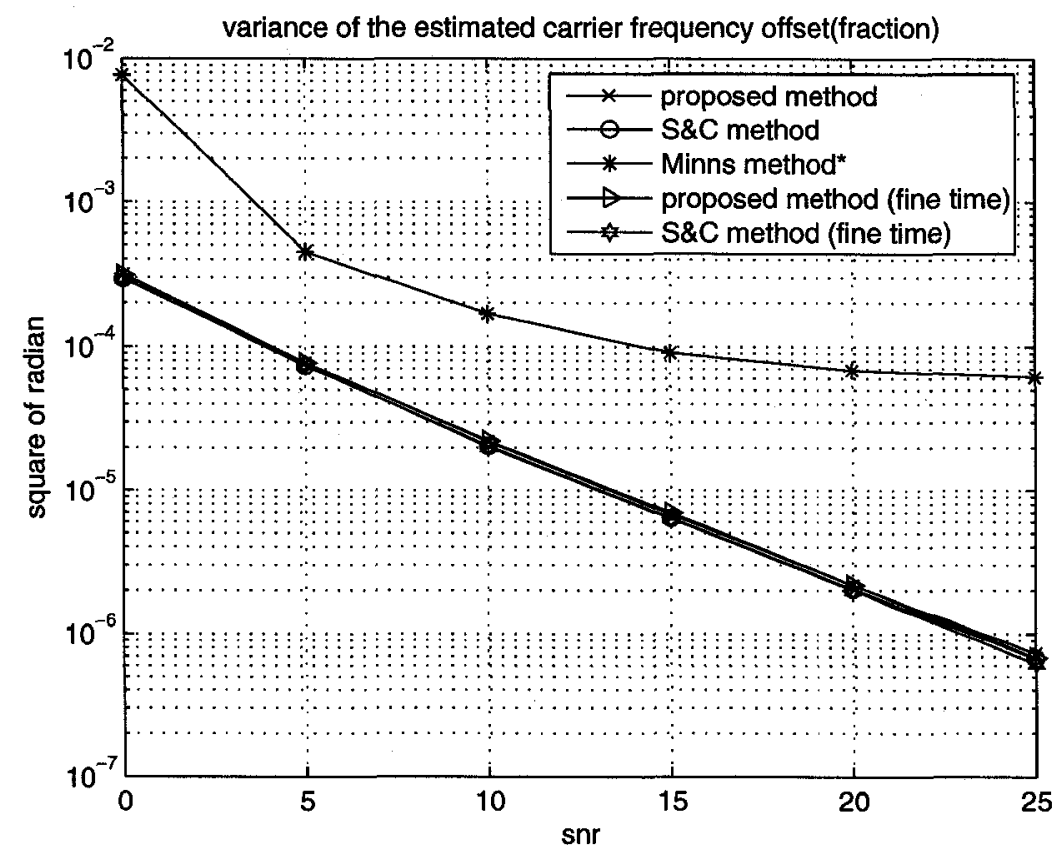

(b) Variance of the fraction part of the CFO

Fig. 5.5 Performance of the CFO (fraction part) estimator in the first ISI channel (*: uses training sequences similar to [7]) 


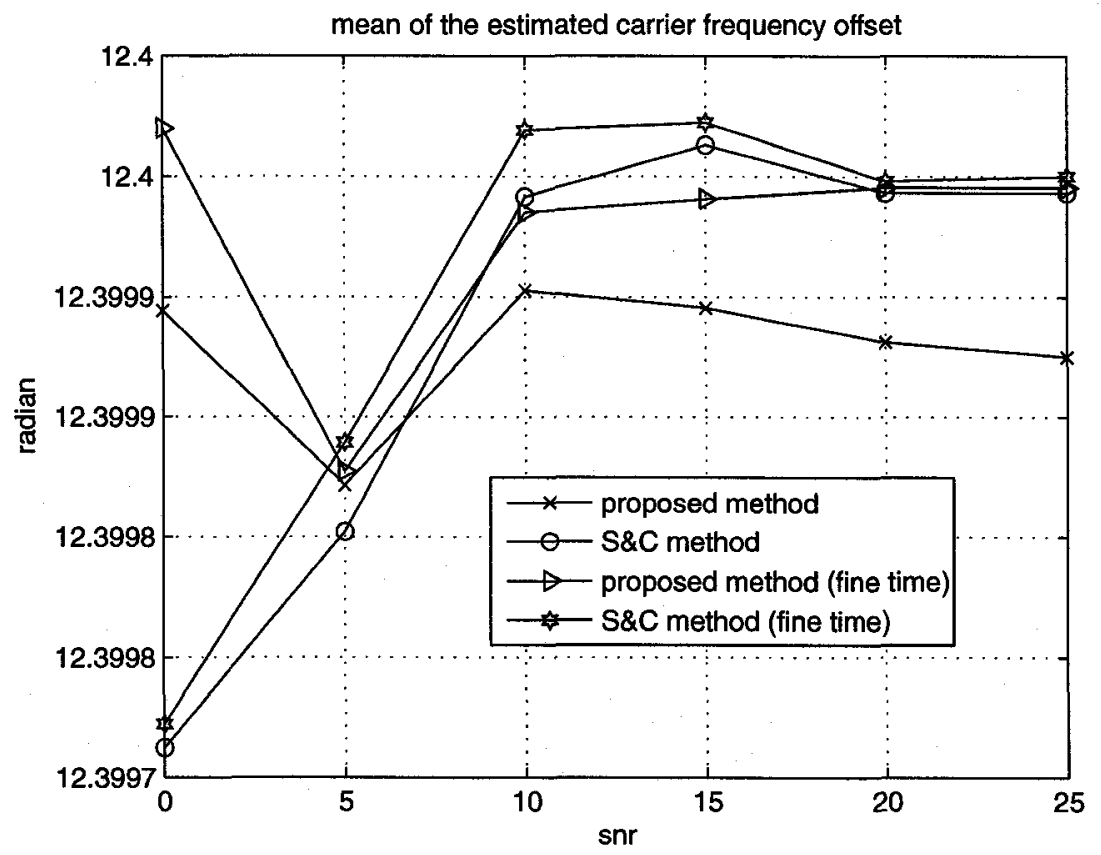

(a) Mean of the CFO

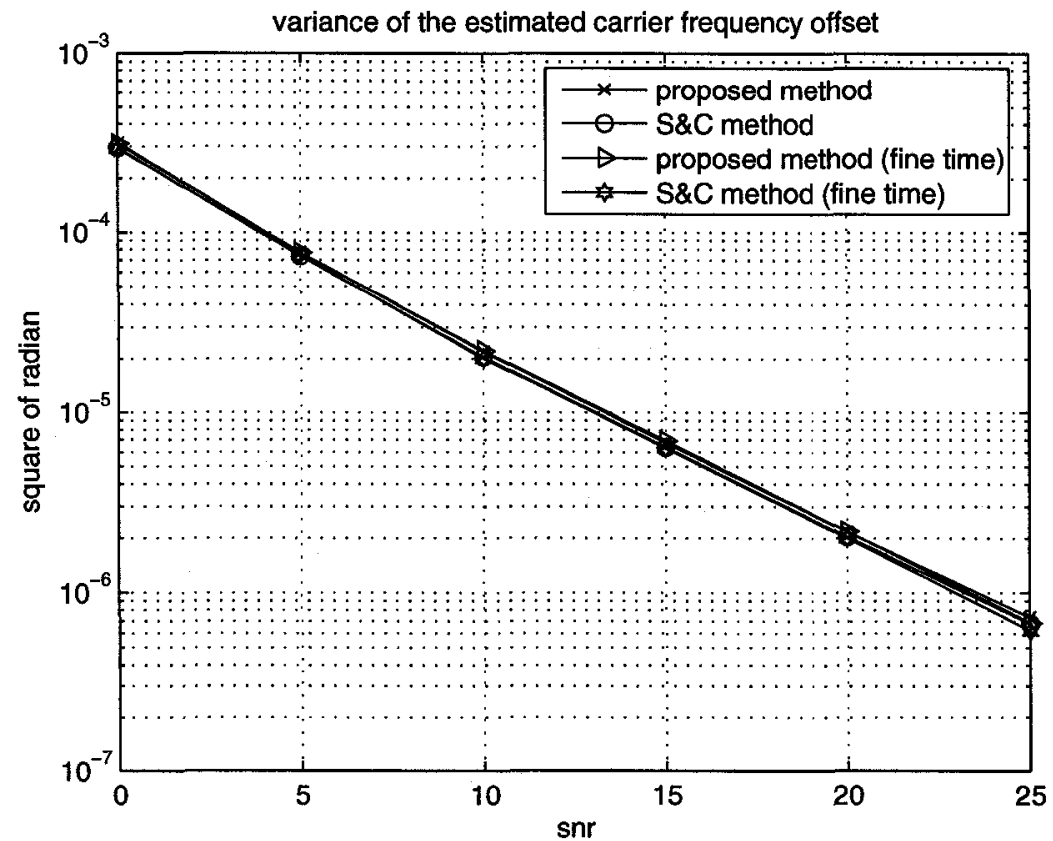

(b) Variance of the CFO

Fig. 5.6 Performance of the CFO estimator in the first ISI channel 


\subsection{Simulation results in the second ISI channel}

Figs. 5.7, 5.8 and 5.9 present the simulation results in terms of the means and variances of the symbol timing and CFO estimators for SC's, Minn's and proposed methods in the second ISI channel.

Fig. 5.7 shows the performance of the symbol timing estimators for different methods in the second ISI channel. Fig. 5.7 shows that effects observed for the first ISI channel are also observed for the second ISI channel. For example, it is seen that the mean values of the proposed coarse timing estimators are quite close to the exact start point and always on the left side of it, whereas they are shifted to the left for SC's method (due to CP) and to the right for Minn's method. The mean values of the proposed and SC fine timing estimators are almost at the exact start point. Fig. 5.7(b) shows that the proposed and SC's fine timing estimators have quite small variance (less than $10^{-4}$ when $S N R \geq 5 \mathrm{~dB}$ for the proposed estimator and less than $10^{-4}$ when $S N R \geq 0 \mathrm{~dB}$ for SC's estimator). Fig. 5.7(b) also shows that all modified methods have smaller variance than the original methods in the second ISI channel as well.

Similarly, as seen in Fig. 5.8 and Fig. 5.9 performance of CFO estimators in the second ISI channel exhibits same trends as for the first ISI channels, except for Minn's CFO mean. The difference of behavior of CFO mean obtained by Minn's method between the first and second ISI channel is in fact due to the different choice of training sequence for the two channels (training sequence similar to [7] for the first ISI channel and Chu sequence for the second ISI channel). Preliminary simulations not shown in this thesis have showed that Minn's method performance is quite sensitive to the choice of training sequences. Fig. 5.9 shows that the proposed CFO estimator has almost the same performance as SC's CFO estimator. The integer part of the 
CFO is estimated correctly for 10,000 runs by SC's and proposed methods for each simulated SNR. From the discussion above, we conclude that the SC's method (fine time) has the best performance in the second ISI channel. Note that it will be shown in section 5.5 that the proposed method (fine time) is better than SC's method (fine time) in Rayleigh fading channels. Therefore, it is to be expected that performance of the proposed method (fine time) might also outperform SC's method (fine time) in other ISI channels. 


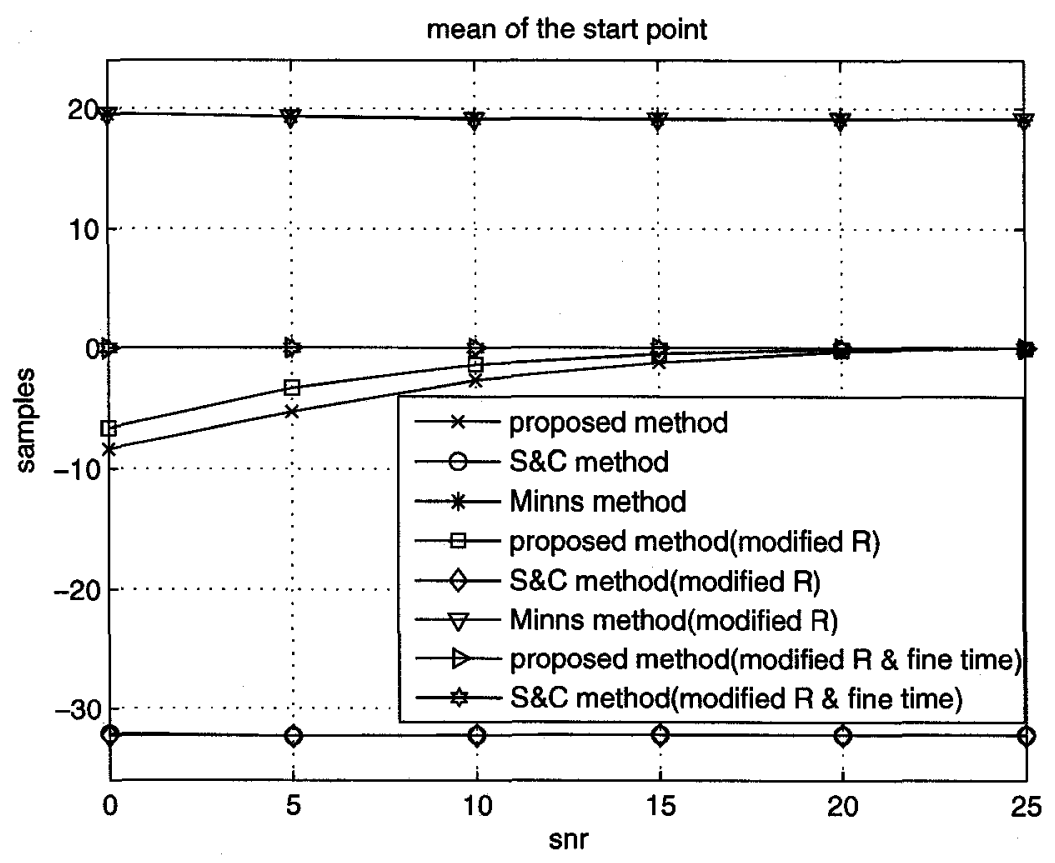

(a) Mean of the start point

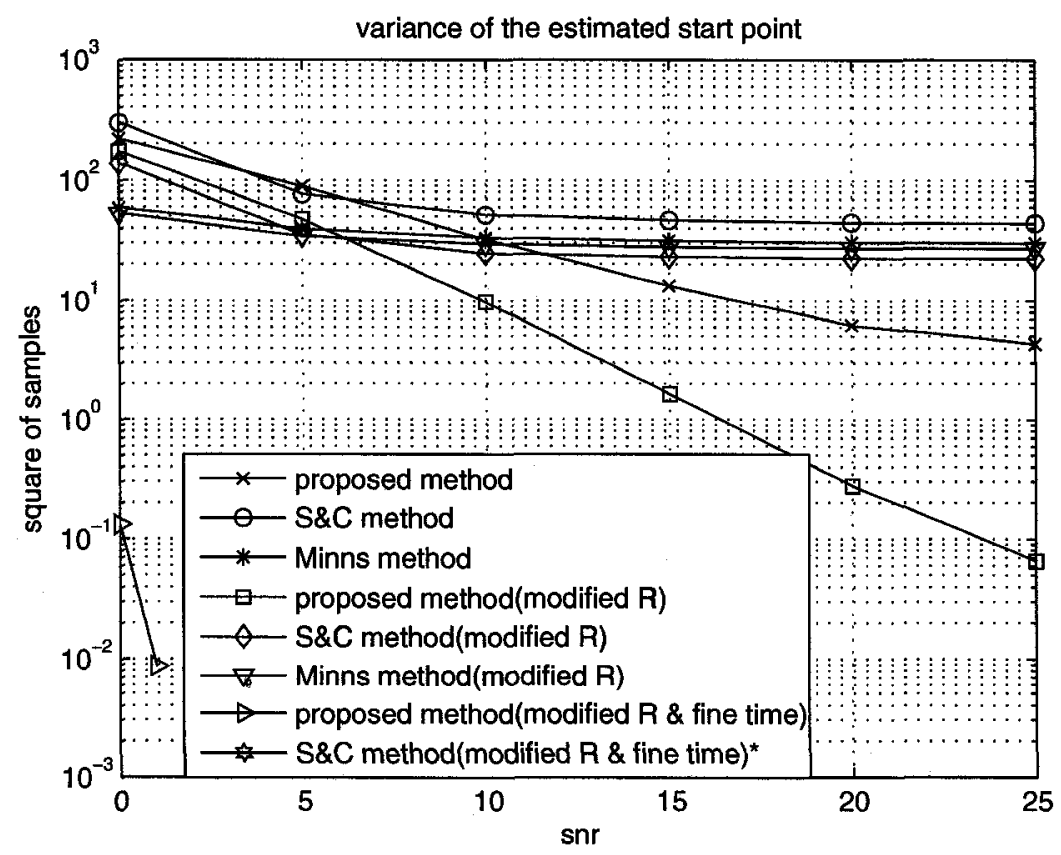

(b) Variance of the start point

Fig. 5.7 Performance of the timing estimator in the second ISI channel (*: The variance of SC's fine timing estimator is less than $10^{-4}$ when $S N R \geq 0 \mathrm{~dB}$ ) 


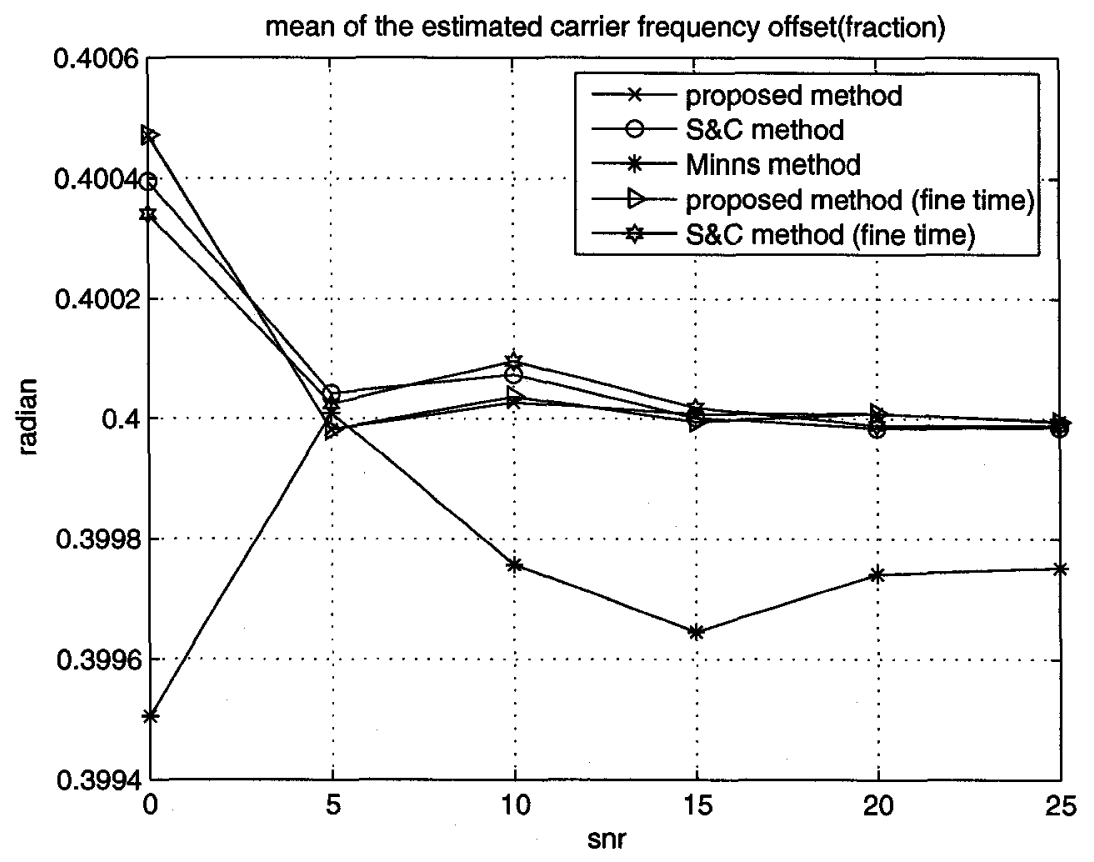

(a) Mean of the fraction part of the CFO

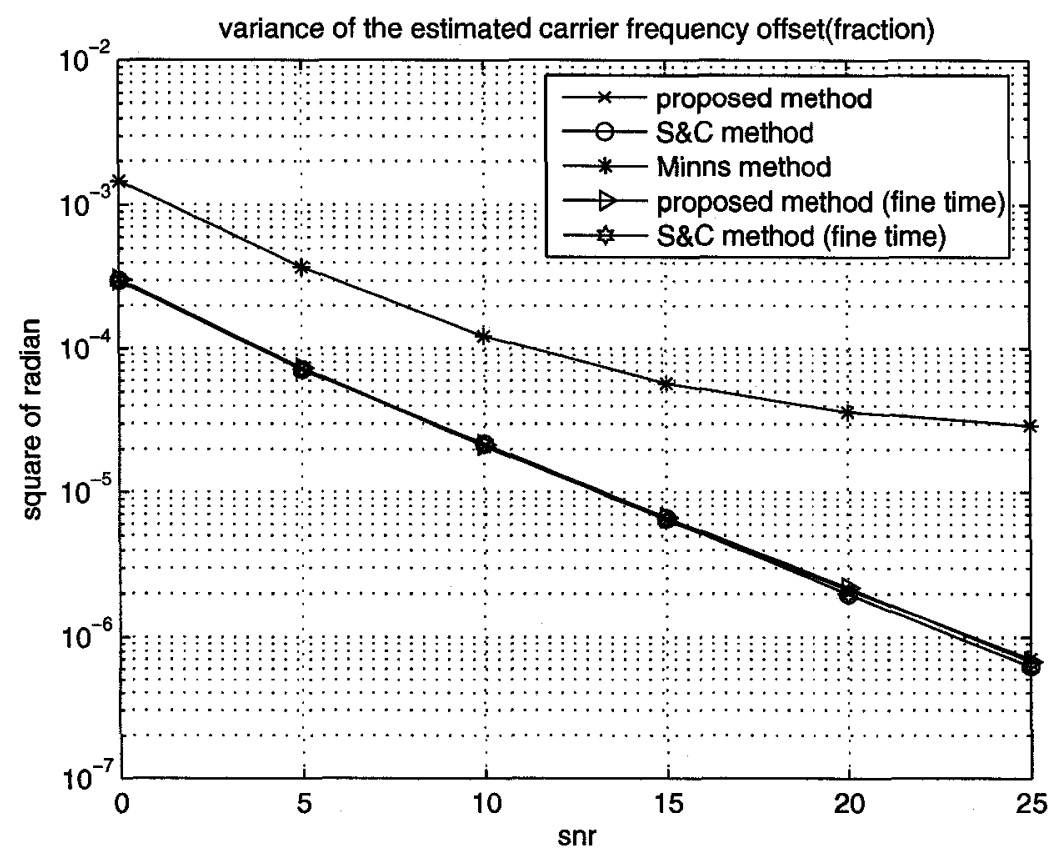

(b) Variance of the fraction part of the CFO

Fig. 5.8 Performance of the CFO (fraction part) estimator in the second ISI channel 


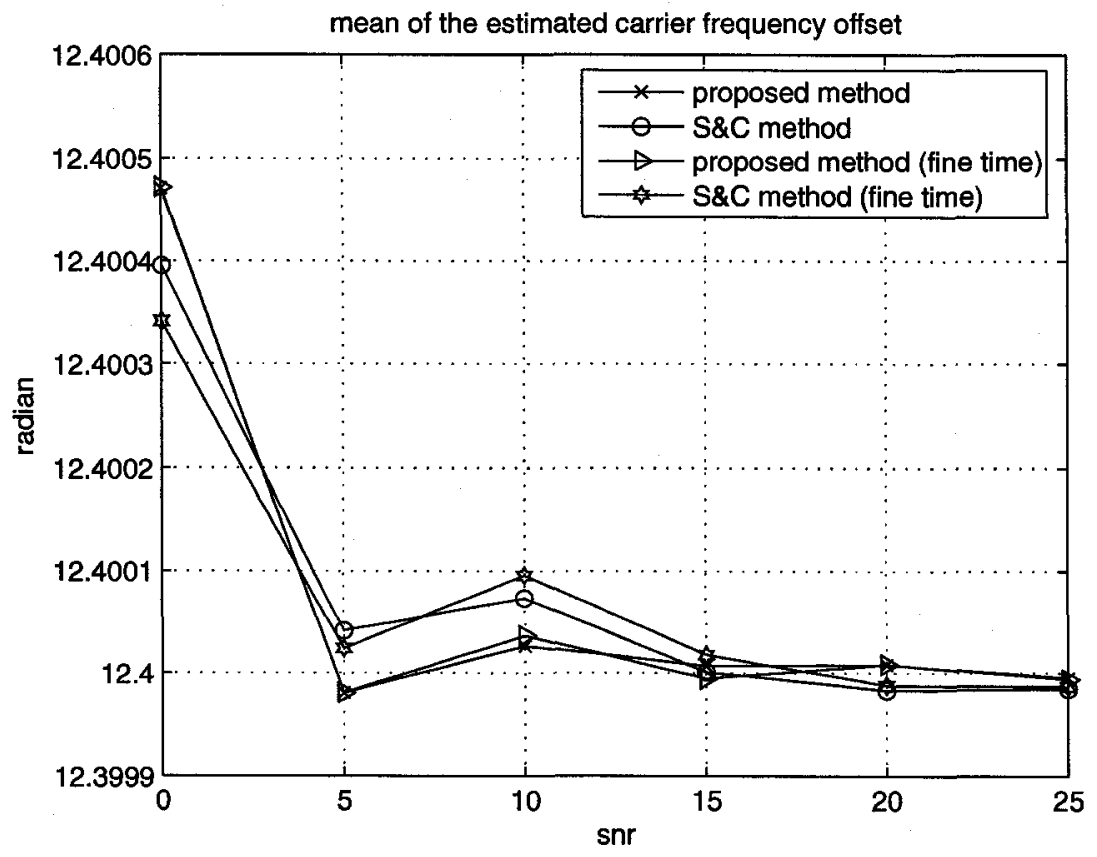

(a) Mean of the CFO

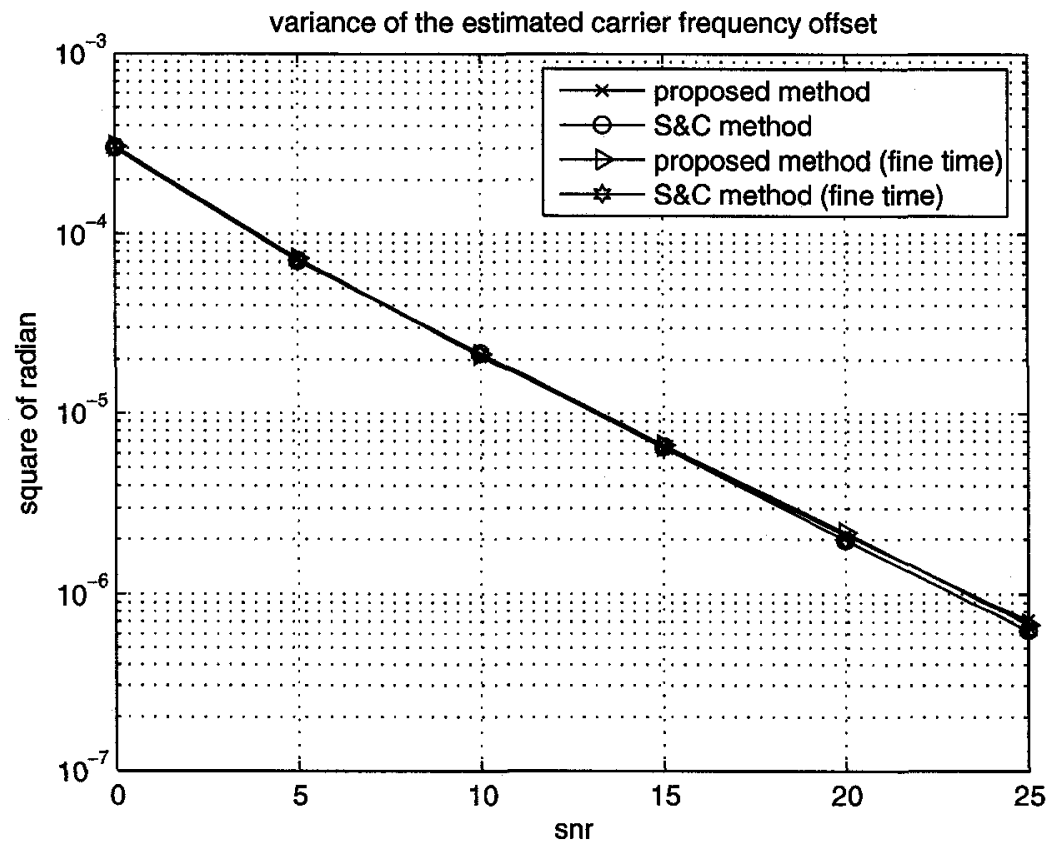

(b) Variance of the CFO

Fig. 5.9 Performance of the CFO estimator in the second ISI channel 


\subsection{Simulation results in the Rayleigh fading channel}

\subsubsection{Simulation results in the time-invariant Rayleigh fading channel}

Fig. 5.10, 5.11 and 5.12 present the simulation results in terms of the means and variances of the symbol timing and CFO estimators for SC's, Minn's and proposed methods in the time-invariant Rayleigh fading channel.

Fig. 5.10 shows the performance of the symbol timing estimators for different methods in the time-invariant Rayleigh fading channel. Fig. 5.10(a) shows that the mean value of the proposed coarse timing estimator is quite closer to the exact start point than SC and Minn's methods. The mean values of the proposed and SC fine timing estimators slightly shift to the right of the exact start point because the first tap might be in deep fade and smaller than the threshold in some simulation runs. Fig. 5.10(b) shows that the proposed coarse timing estimator has quite small variance in the time-invariant Rayleigh fading channel. If we need a more accurate estimator, we can implement fine timing synchronization and the price is the computational complexity. The proposed fine timing estimator has better performance (in terms of variance) than SC's fine timing estimator at low SNR due to the FlP-based preamble. Similar to AWGN and ISI channels results, Fig. 5.10(b) also shows that all modified methods perform better than the original methods.

Fig. 5.11 and Fig. 5.12 show the performance of the CFO estimators for different methods in the time-invariant Rayleigh fading channel. The variance of the proposed CFO estimator is similar to SC's CFO estimator at low SNR and slightly bigger at high SNR. Using the fine time point or the coarse time point to estimate CFO yields almost same performance for both the proposed and SC's methods. Minn's CFO estimator has the worst performance among all three CFO (fraction part) estimators due to the short length of its repeated part. The integer part of the CFO is estimated 


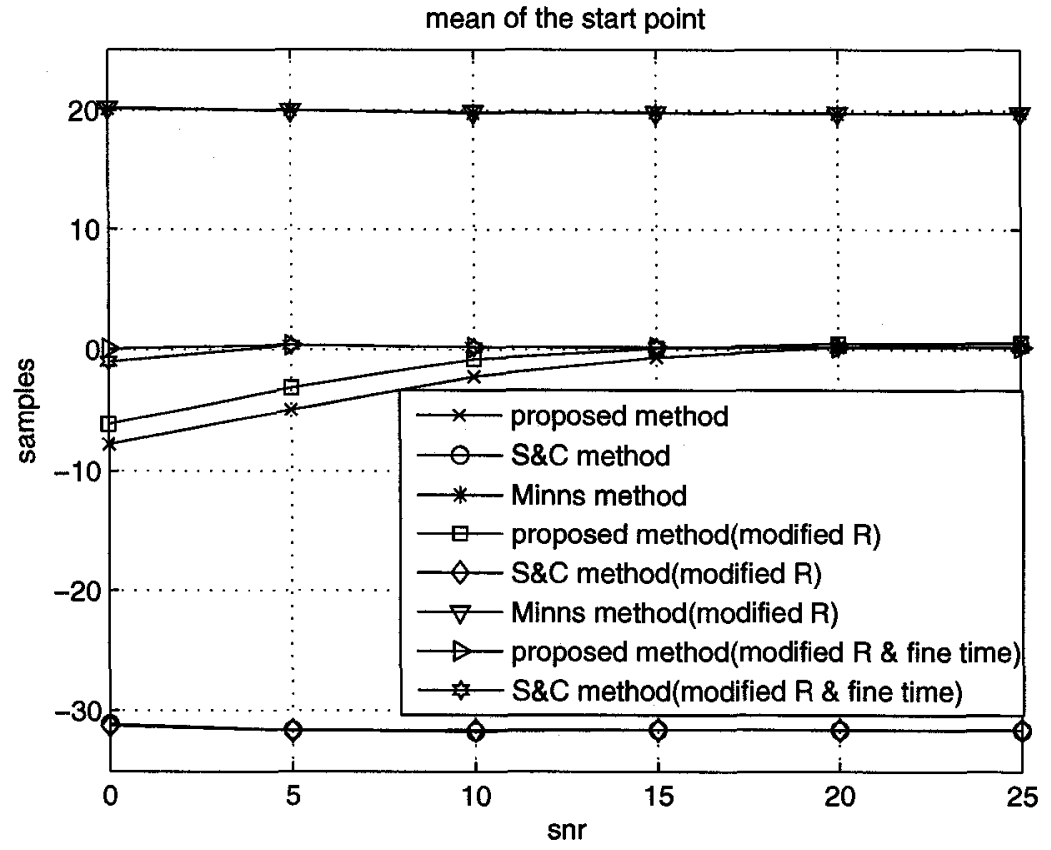

(a) Mean of the start point

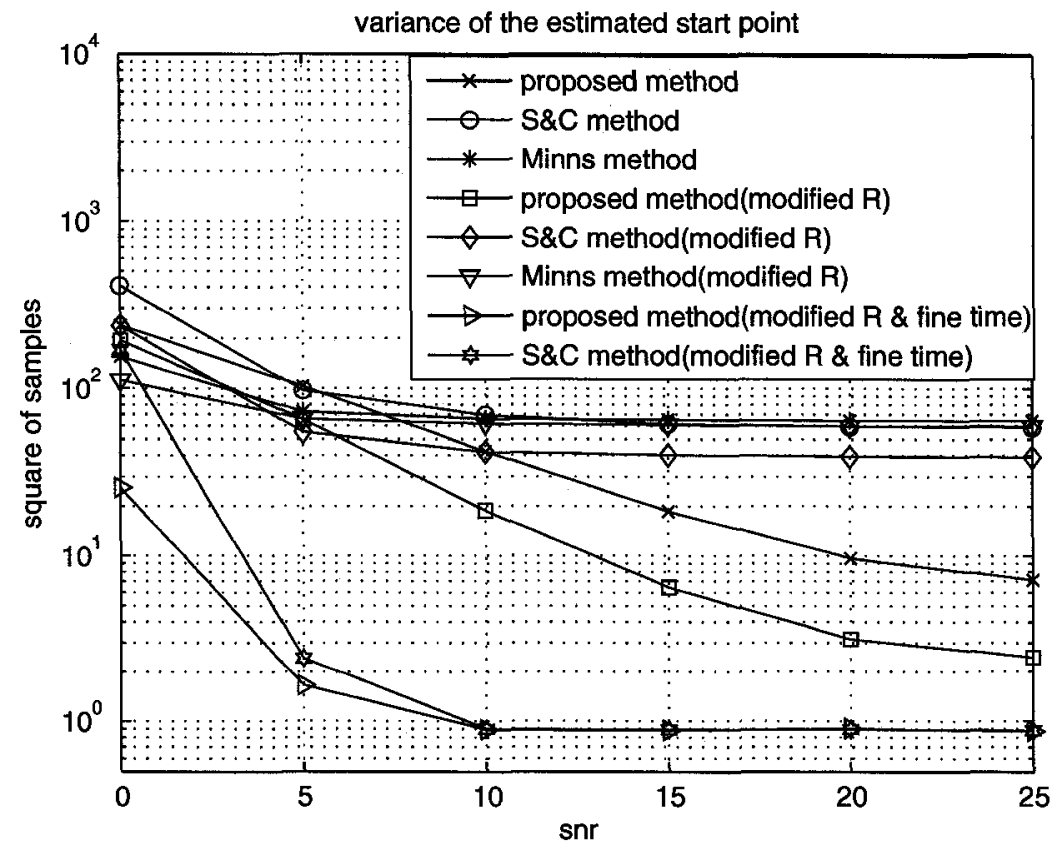

(b) Variance of the start point

Fig. 5.10 Performance of the timing estimator in the time-invariant Rayleigh fading channel 


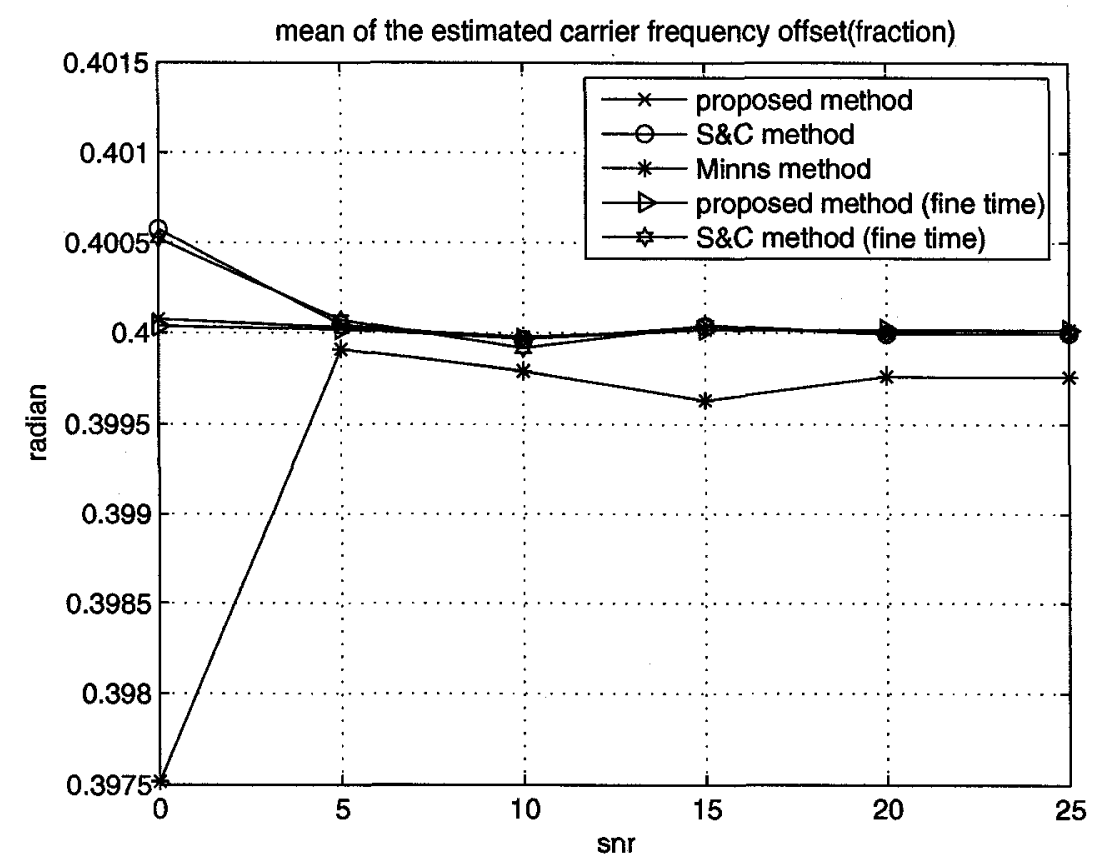

(a) Mean of the fraction part of the CFO

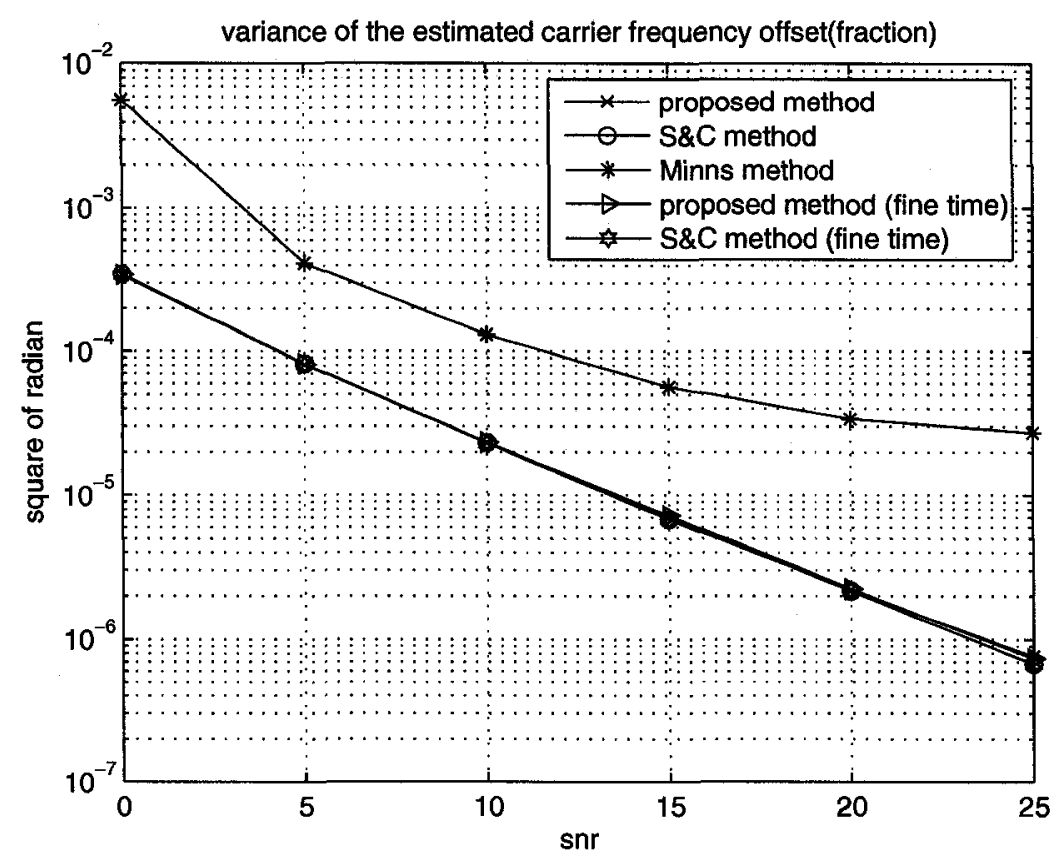

(b) Variance of the fraction part of the CFO

Fig. 5.11 Performance of the CFO (fraction part) estimator in the time-invariant Rayleigh fading channel 


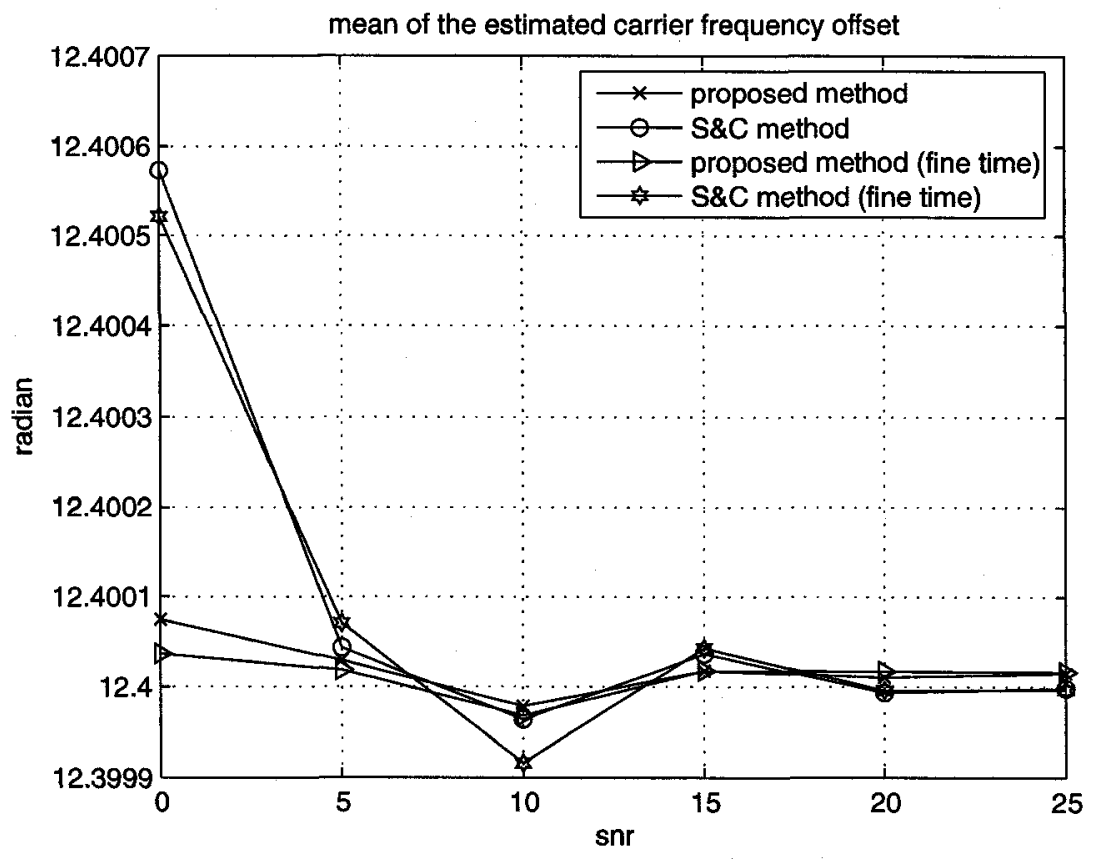

(a) Mean of the CFO

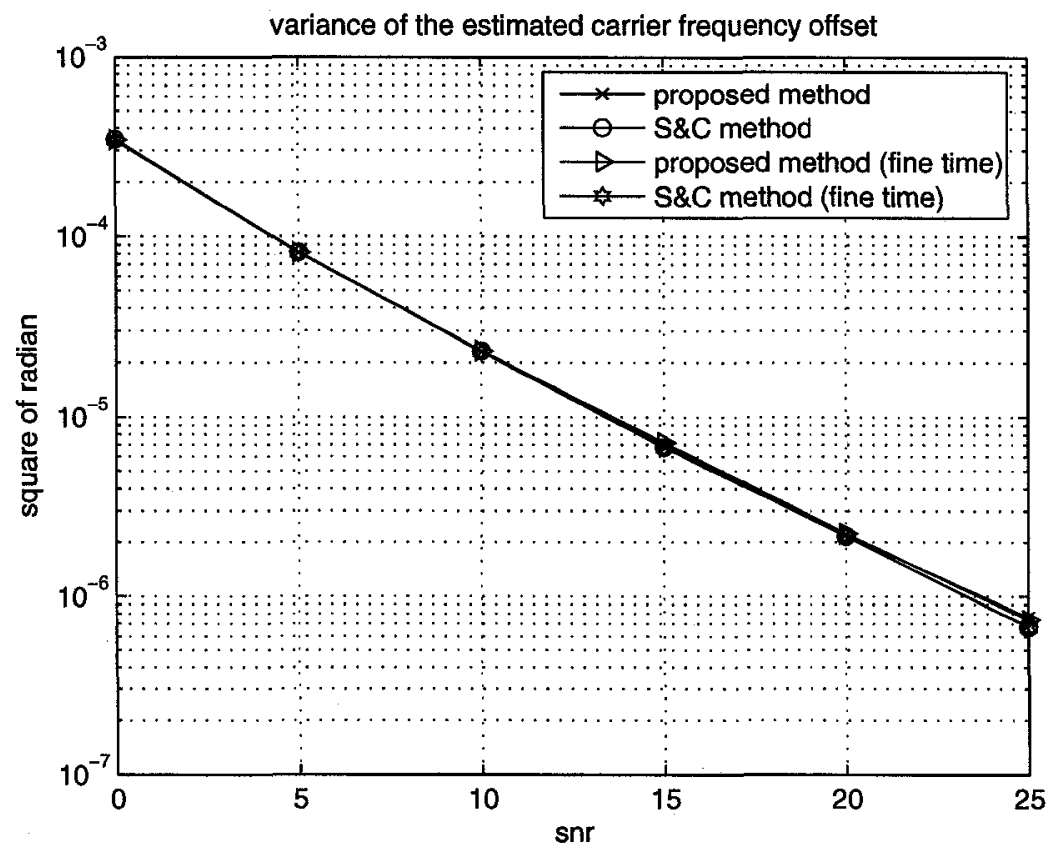

(b) Variance of the CFO

Fig. 5.12 Performance of the CFO estimator in the time-invariant Rayleigh fading channel 
correctly for 10,000 runs by SC's and proposed methods for each simulated SNR.

From the discussion above, we conclude that the proposed method (fine time) has the best performance in the time-invariant Rayleigh fading channel.

\subsubsection{Simulation results in the time-variant Rayleigh fading channel}

Figs. 5.13-5.21 present the simulation results in terms of the means and variances of the symbol timing and CFO estimators for SC's, Minn's and proposed methods in the time-variant Rayleigh fading channel with different maximum Doppler frequencies. Figs. 5.13-5.15, Figs. 5.16-5.18 and Figs. 5.19-5.21 presents results for a low vehicle speed $(v=10 \mathrm{~km} / \mathrm{hr})$, a moderate vehicle speed $(v=70 \mathrm{~km} / \mathrm{hr})$ and a high vehicle speed $(v=250 \mathrm{~km} / \mathrm{hr}$ ) respectively. Figs. 5.13-5.15 results (mean and variance of the estimated start point, variance of the estimated CFO) are very close to the results obtained in Figs. 5.10-5.12 for the time invariant Rayleigh fading channel, which was to be expected for such a low vehicle speed $(v=10 \mathrm{~km} / \mathrm{hr})$.

For each maximum Doppler frequency, the proposed fine timing estimator has the best performance. The variance of the proposed CFO estimator is similar to SC's CFO estimator one at low SNR and slightly bigger at high SNR. Performance when the fine time point or the coarse time point is used to estimate CFO is almost the same since $N=1024$ is large. Minn's CFO estimator has the worse performance among all three CFO estimators due to the short length of its repeated part.

In Fig. 5.22 and Fig. 5.23, we compare the performance of the proposed timing and CFO estimators with different maximum Doppler frequencies. The timing estimators have almost the same performance when the maximum Doppler frequency changes. When the maximum Doppler frequency increases, the variance of CFO estimator also increases. The CFO estimator is more sensitive to the rate of the channel change. The integer part of the CFO is estimated correctly for 10,000 runs by SC's and proposed 


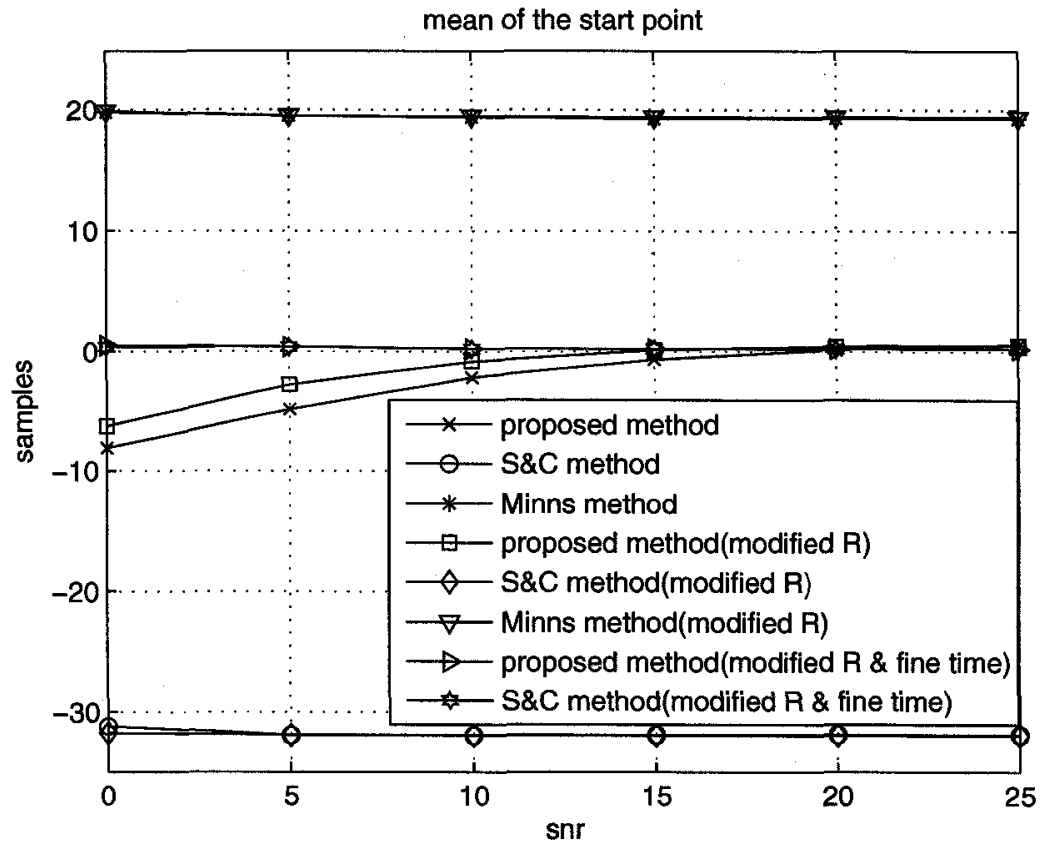

(a) Mean of the start point

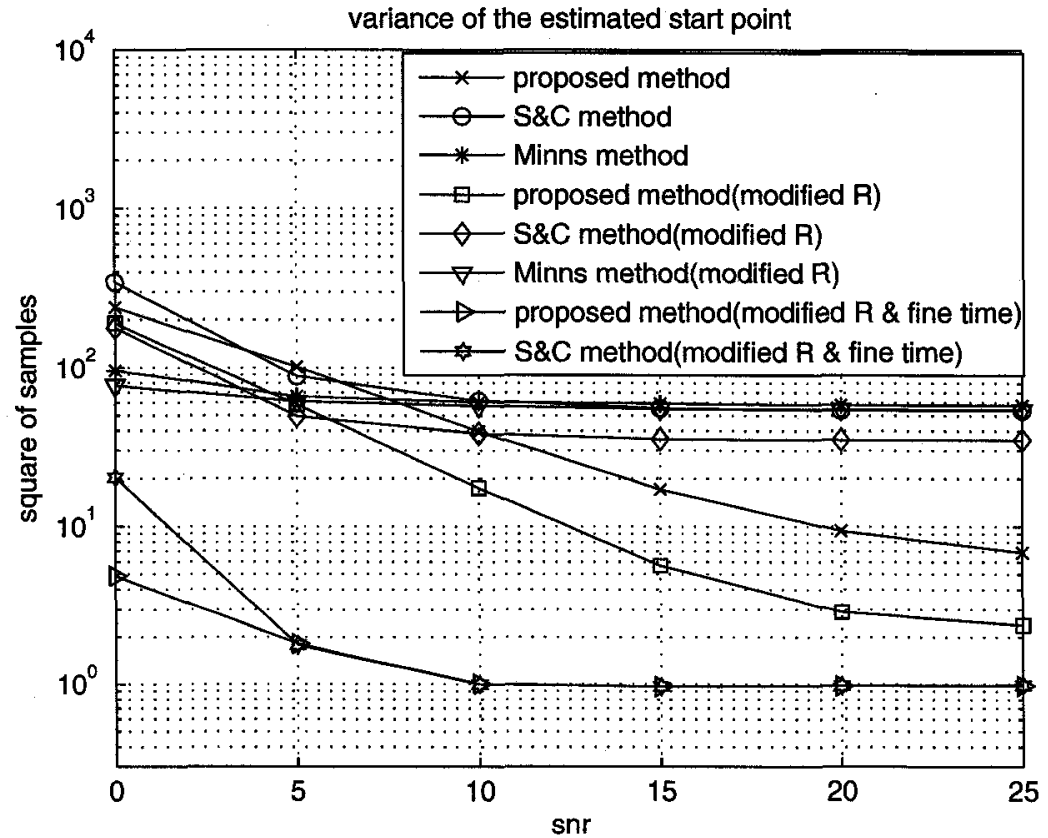

(b) Variance of the start point

Fig. 5.13 Performance of the timing estimator in the time-variant Rayleigh fading channel, $v=10 \mathrm{~km} / \mathrm{hr}, f_{m}=46.3 \mathrm{H}_{z}$ 


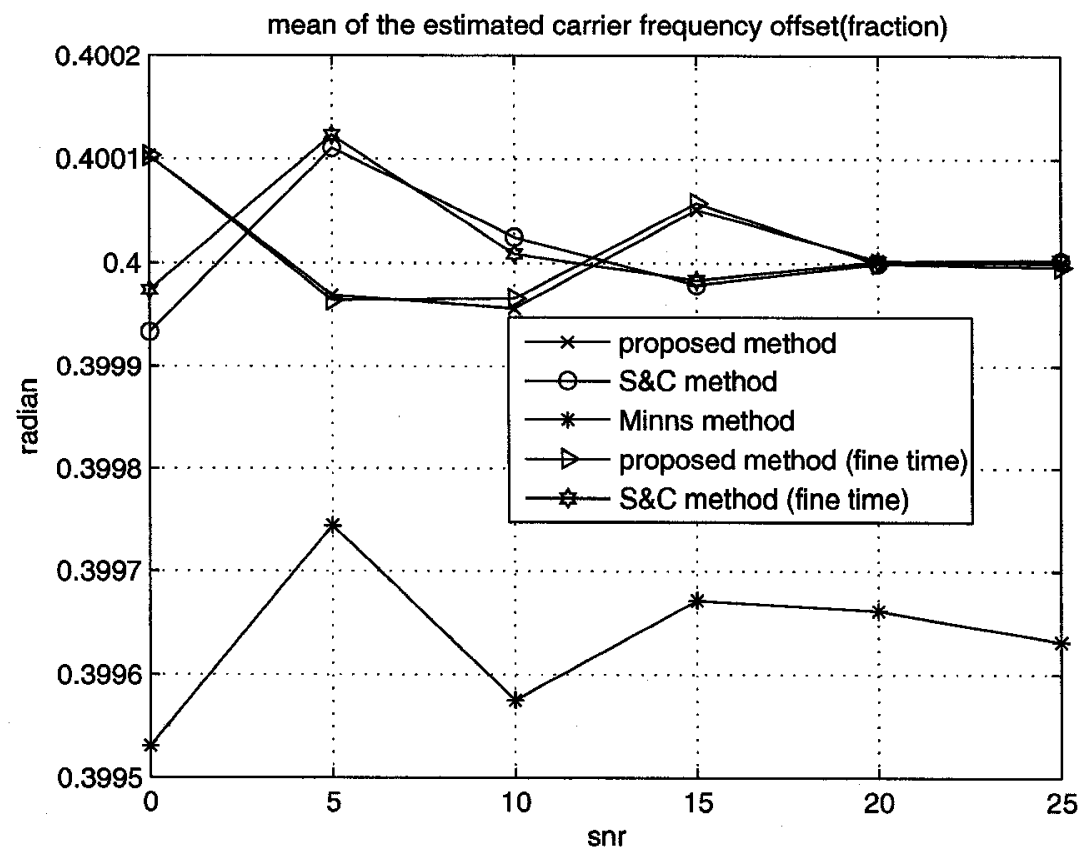

(a) Mean of the fraction part of the CFO

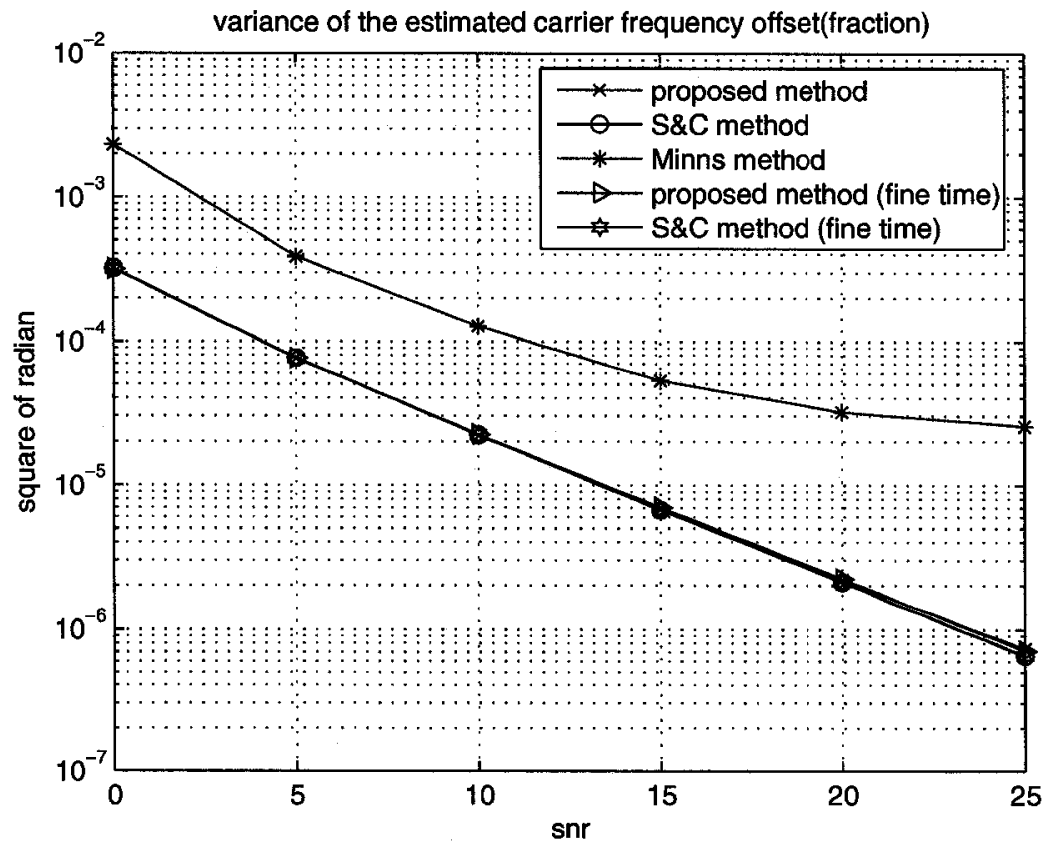

(b) Variance of the fraction part of the CFO

Fig. 5.14 Performance of the CFO (fraction part) estimator in the time-variant Rayleigh fading channel, $v=10 \mathrm{~km} / \mathrm{hr}, f_{m}=46.3 H_{z}$ 


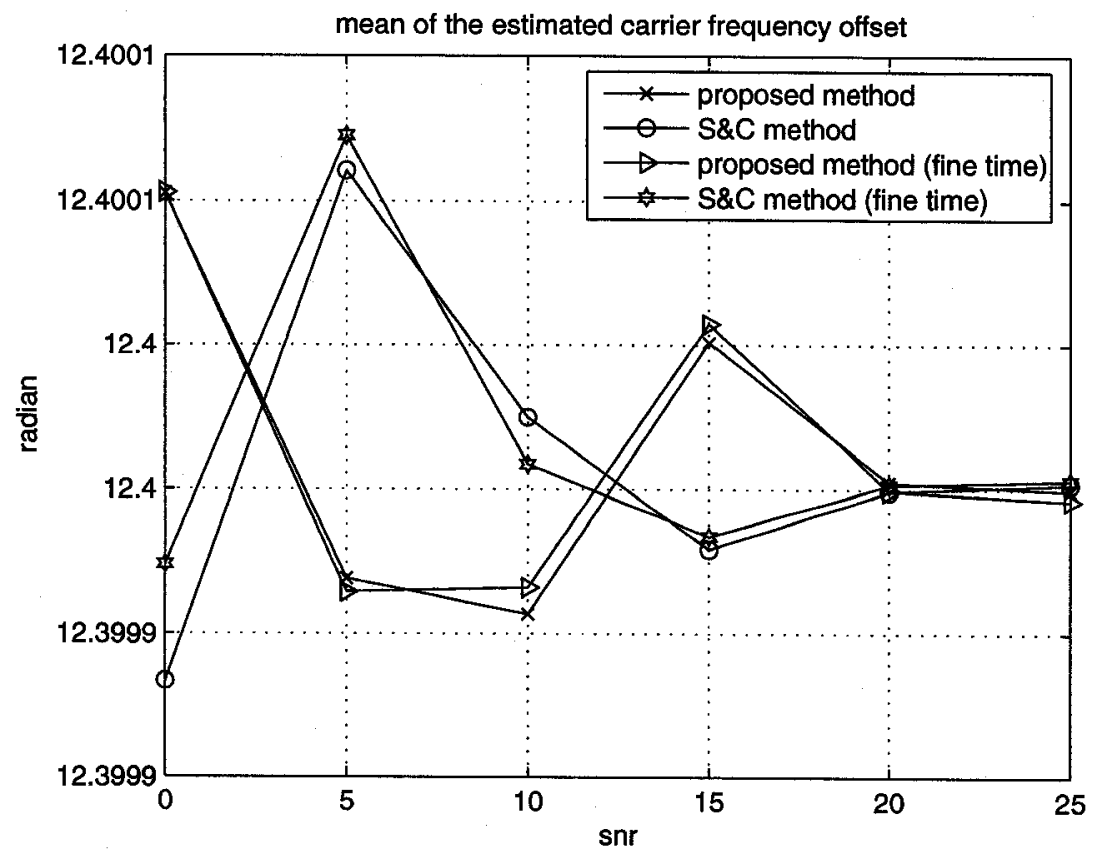

(a) Mean of the CFO

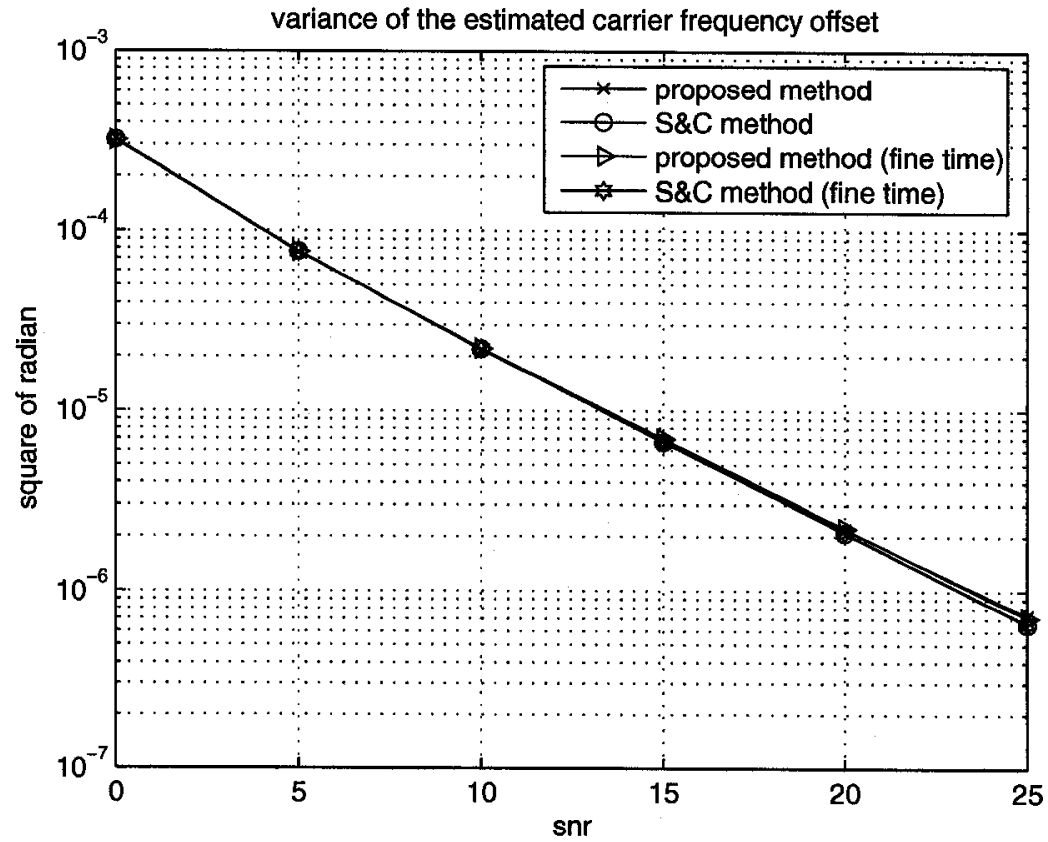

(b) Variance of the CFO

Fig. 5.15 Performance of the CFO estimator in the time-variant Rayleigh fading channel, $v=10 \mathrm{~km} / \mathrm{hr}, f_{m}=46.3 H_{z}$ 


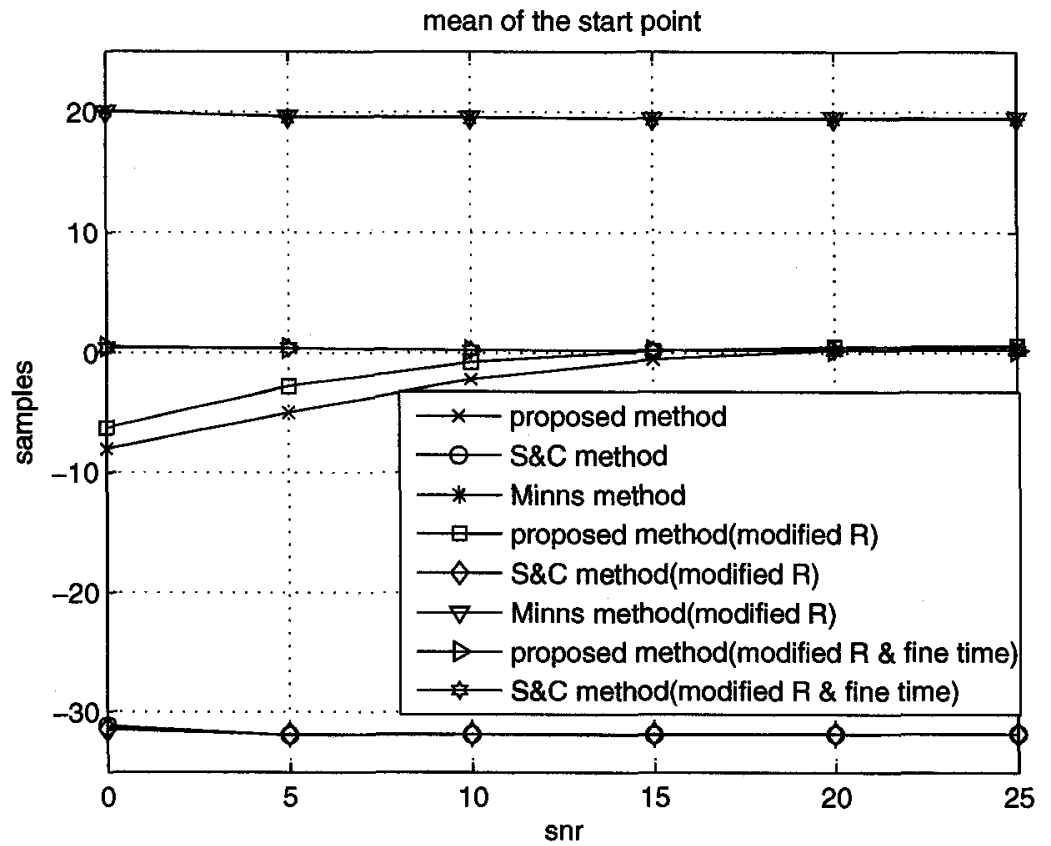

(a) Mean of the start point

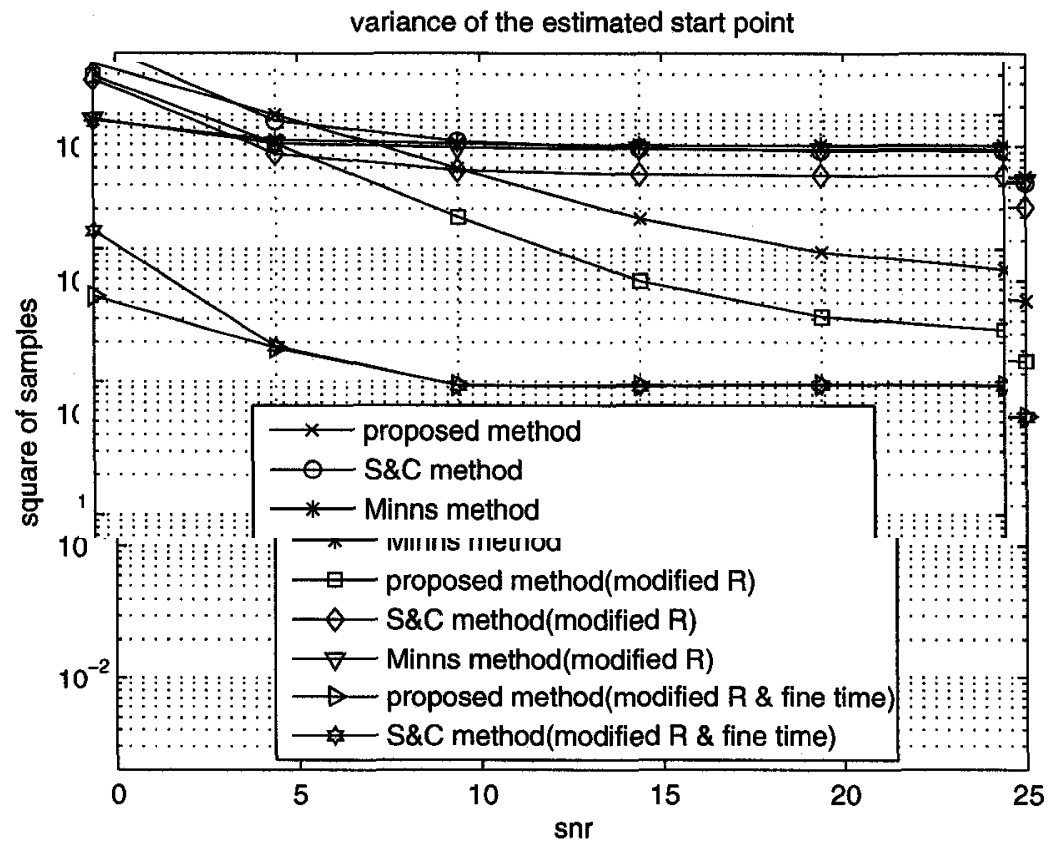

(b) Variance of the start point

Fig. 5.16 Performance of the timing estimator in the time-variant Rayleigh fading channel, $v=70 \mathrm{~km} / \mathrm{hr}, f_{m}=324.1 \mathrm{H}_{z}$ 


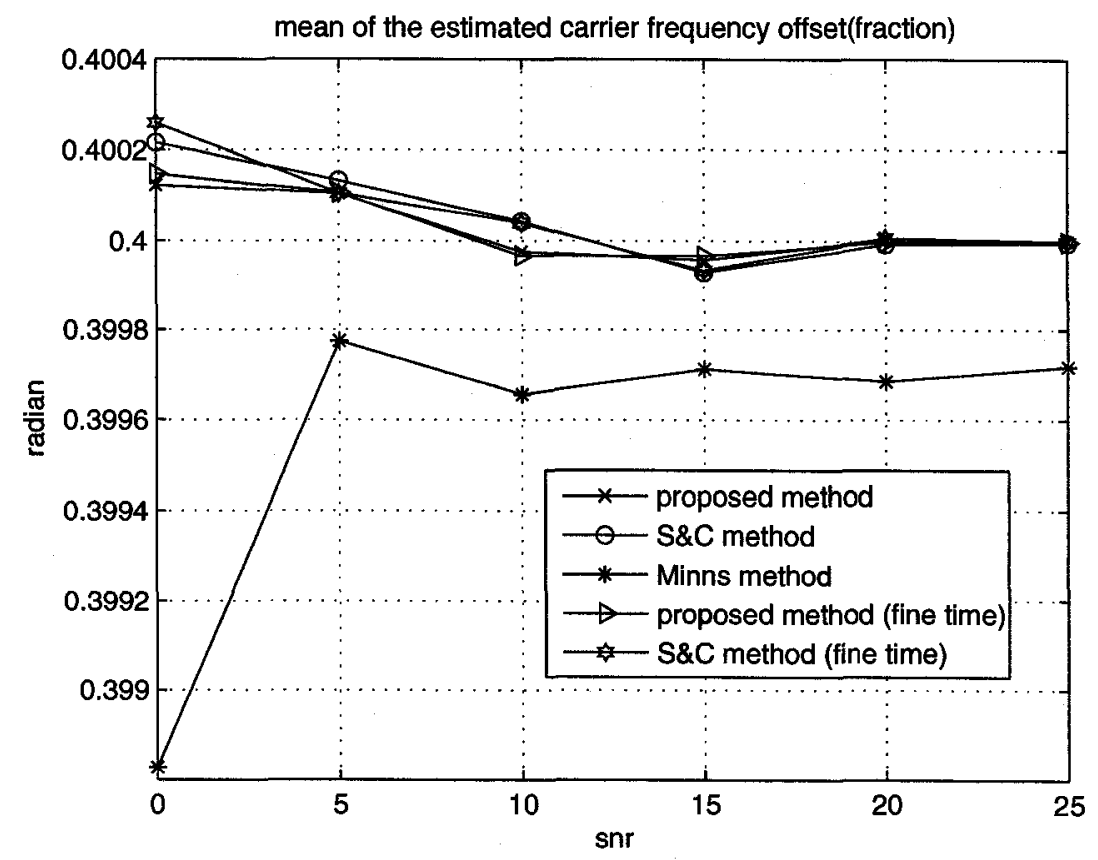

(a) Mean of the fraction part of the CFO

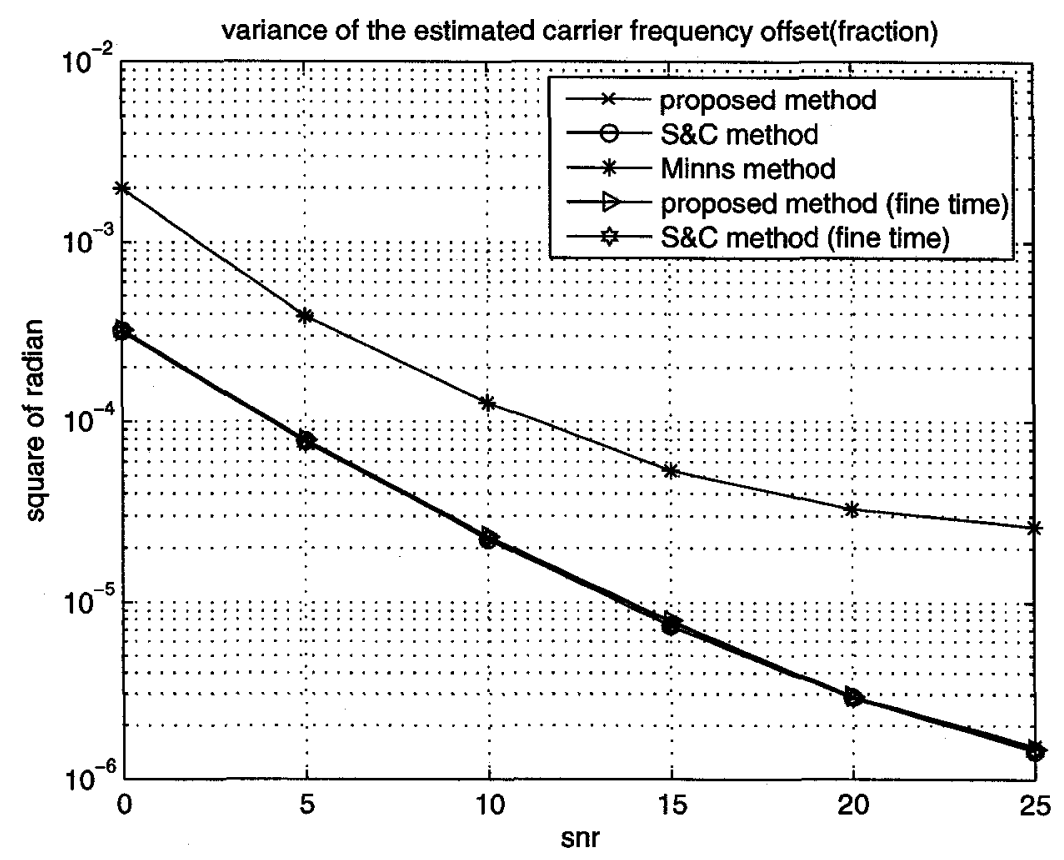

(b) Variance of the fraction part of the CFO

Fig. 5.17 Performance of the CFO (fraction part) estimator in the time-variant Rayleigh fading channel, $v=70 \mathrm{~km} / \mathrm{hr}, f_{m}=324.1 H_{z}$ 


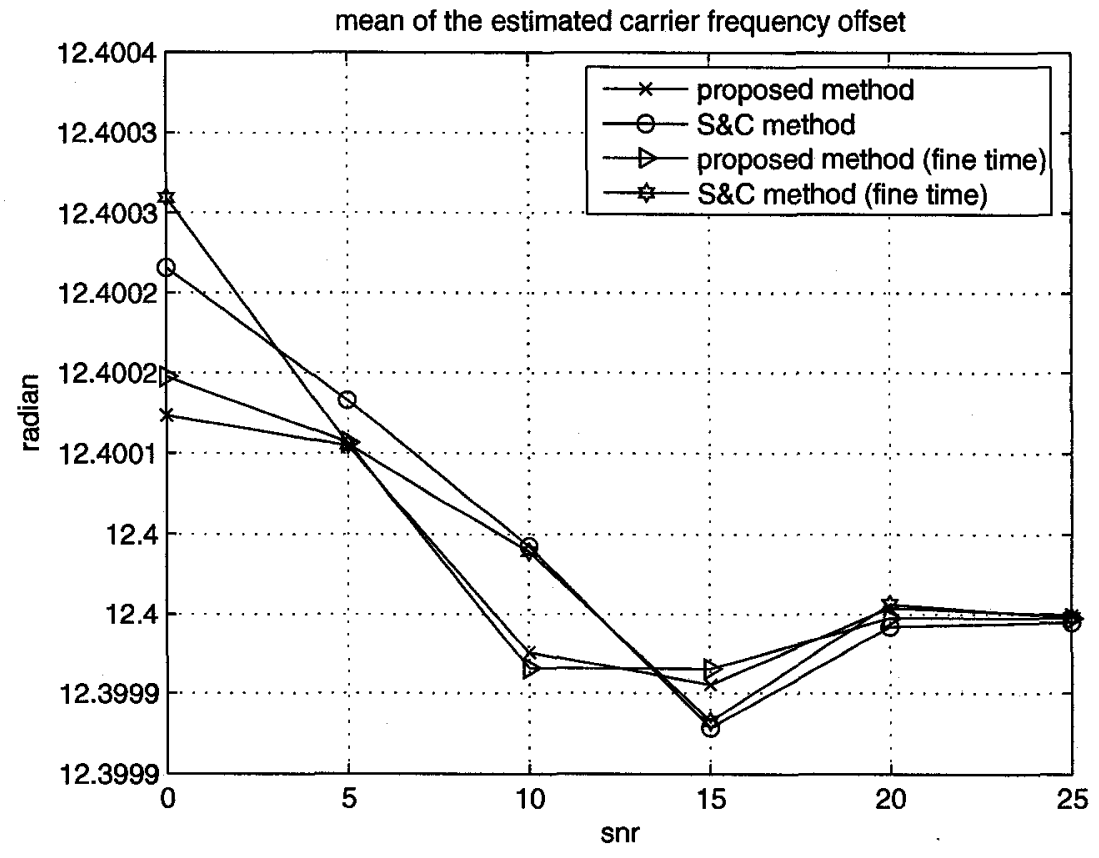

(a) Mean of the CFO

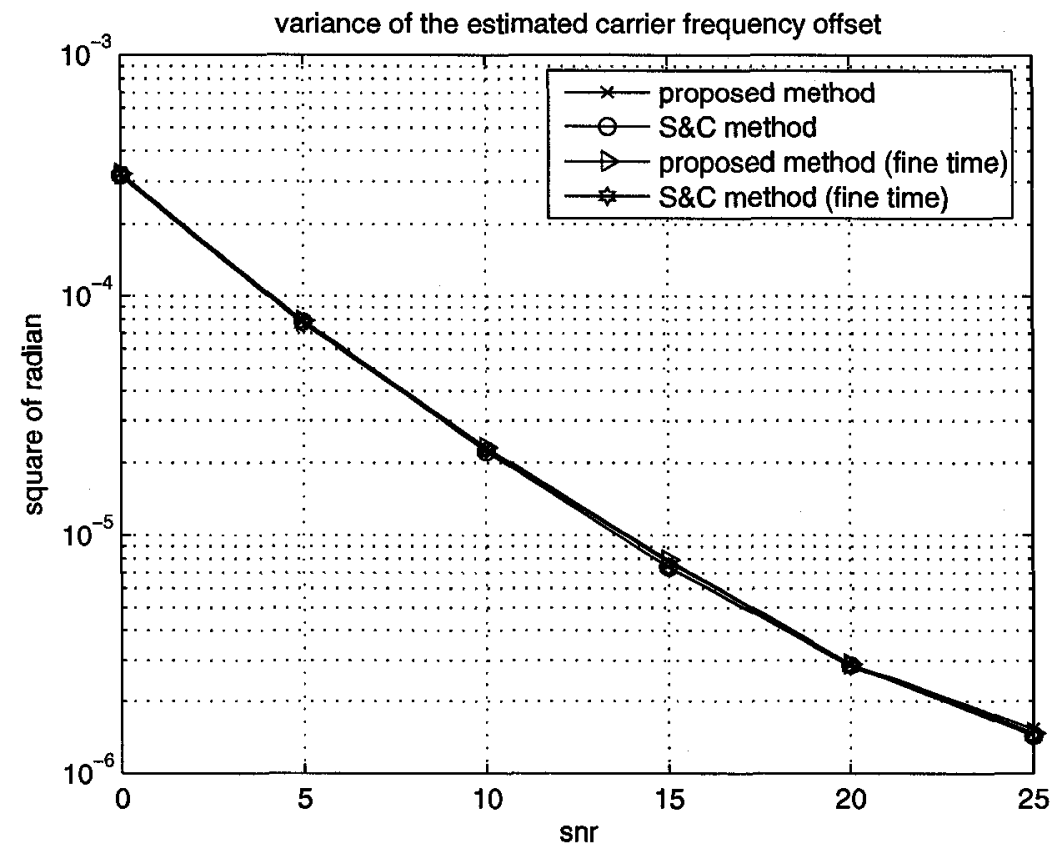

(b) Variance of the CFO

Fig. 5.18 Performance of the CFO estimator in the time-variant Rayleigh fading channel, $v=70 \mathrm{~km} / \mathrm{hr}, f_{m}=324.1 \mathrm{H}_{z}$ 


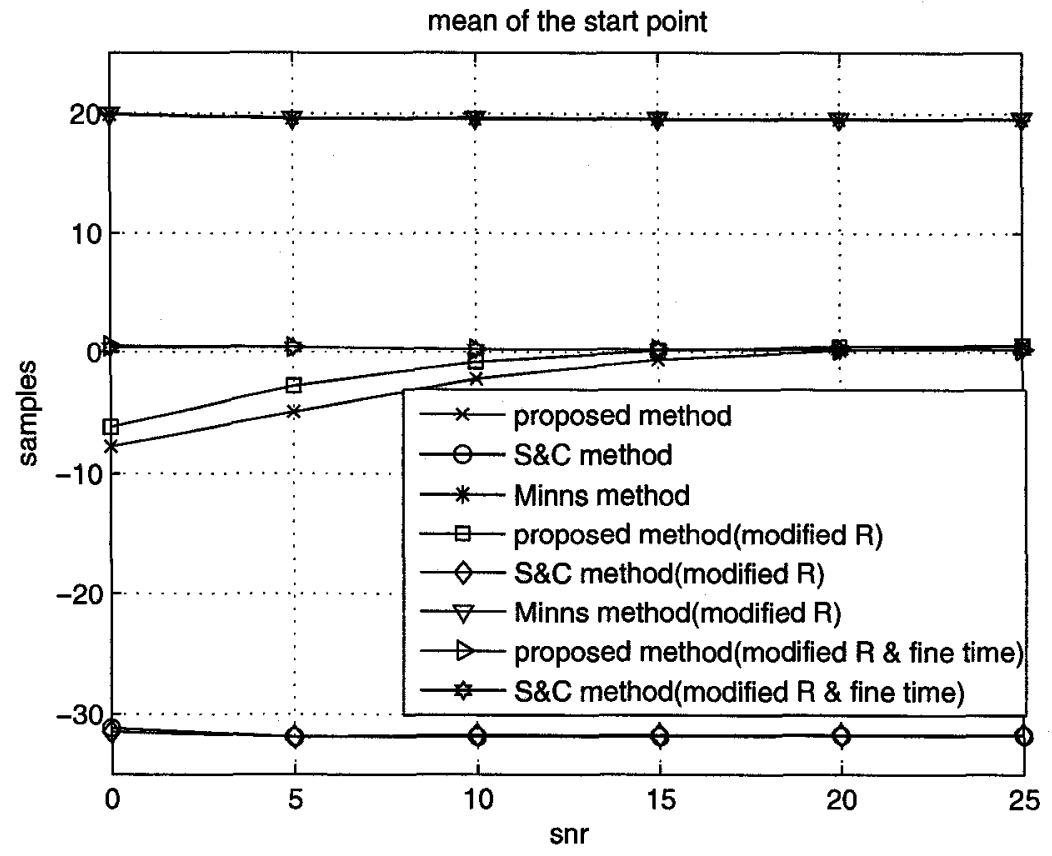

(a) Mean of the start point

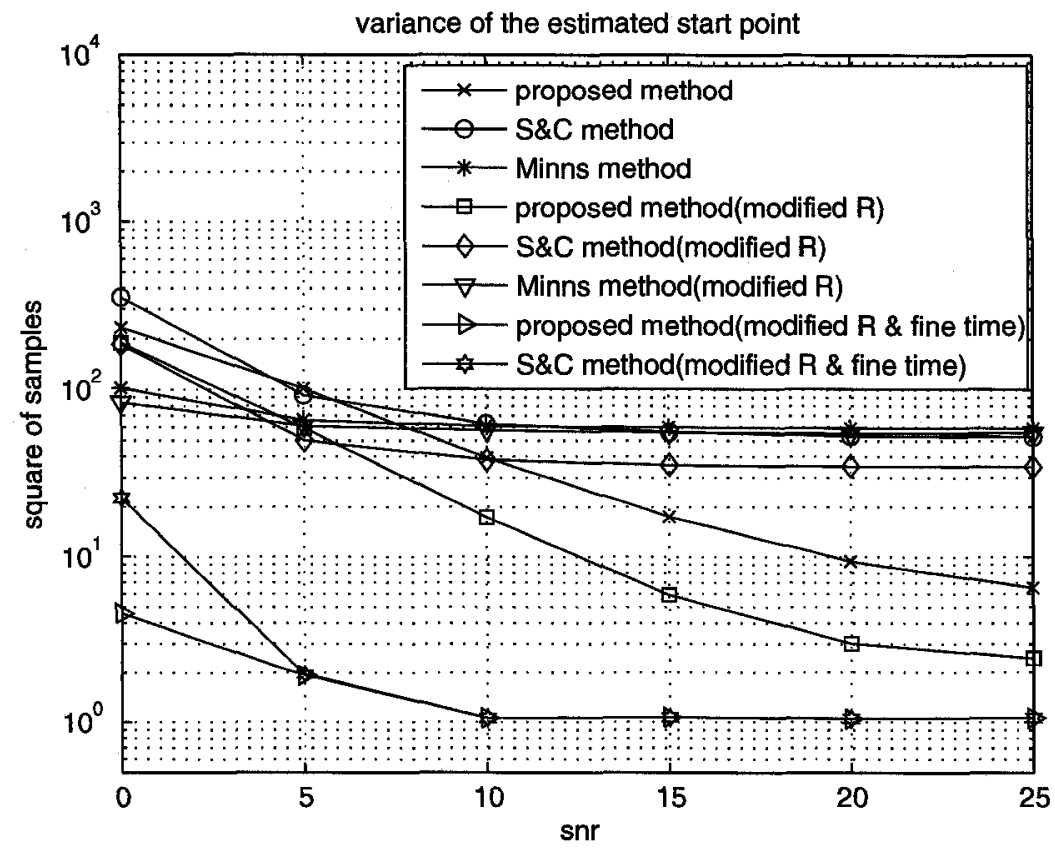

(b) Variance of the start point

Fig. 5.19 Performance of the timing estimator in the time-variant Rayleigh fading channel, $v=250 \mathrm{~km} / \mathrm{hr}, f_{m}=1157.4 H_{z}$ 


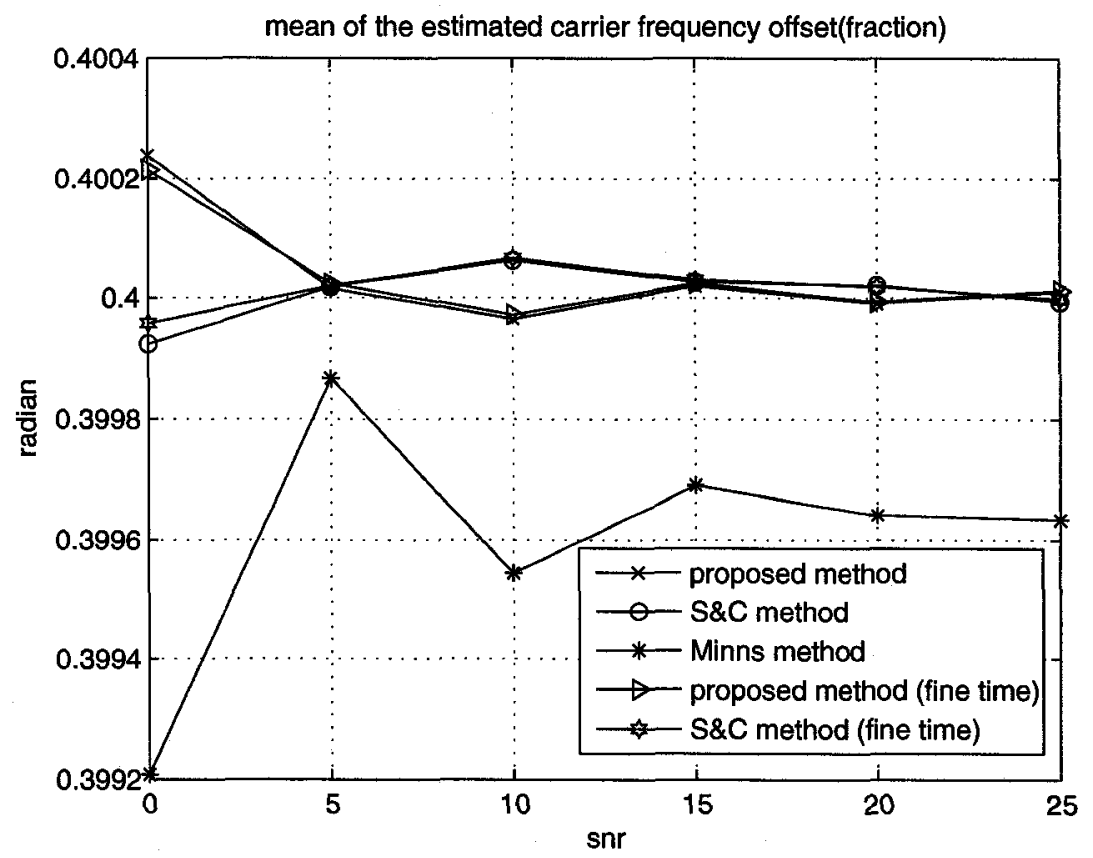

(a) Mean of the fraction part of the CFO

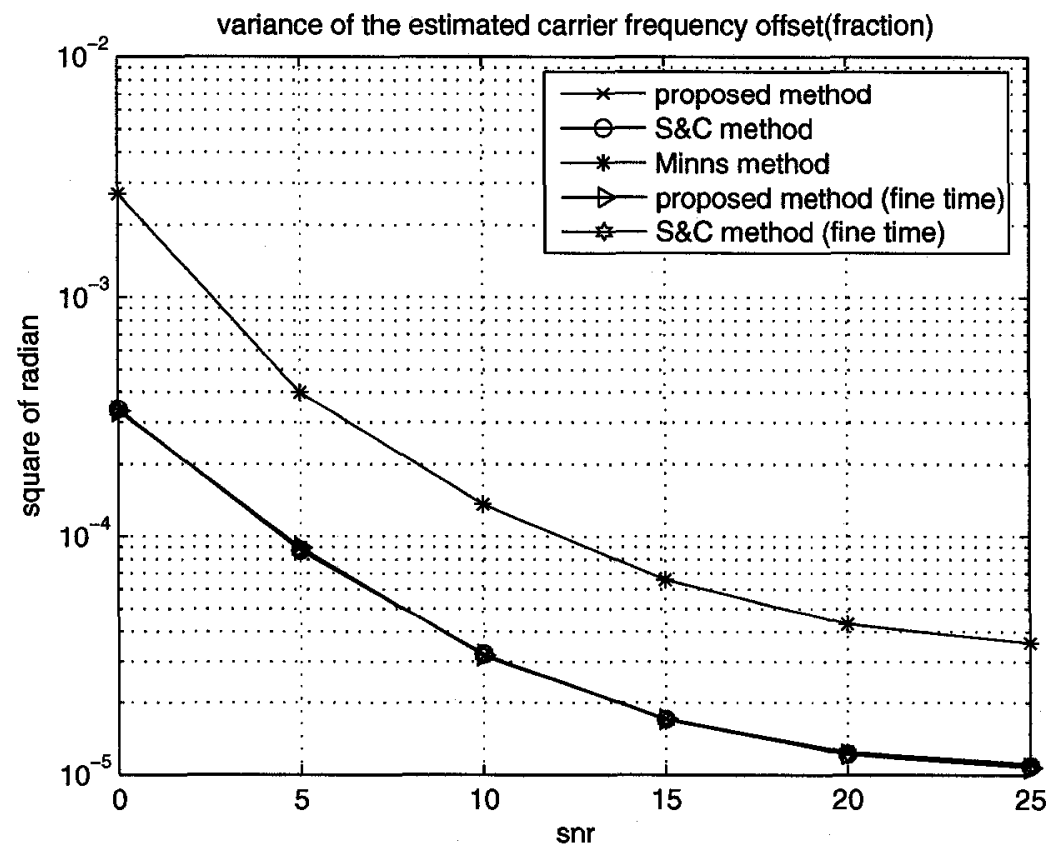

(b) Variance of the fraction part of the CFO

Fig. 5.20 Performance of the CFO (fraction part) estimator in the time-variant Rayleigh fading channel, $v=250 \mathrm{~km} / \mathrm{hr}, f_{m}=1157.4 H_{z}$ 


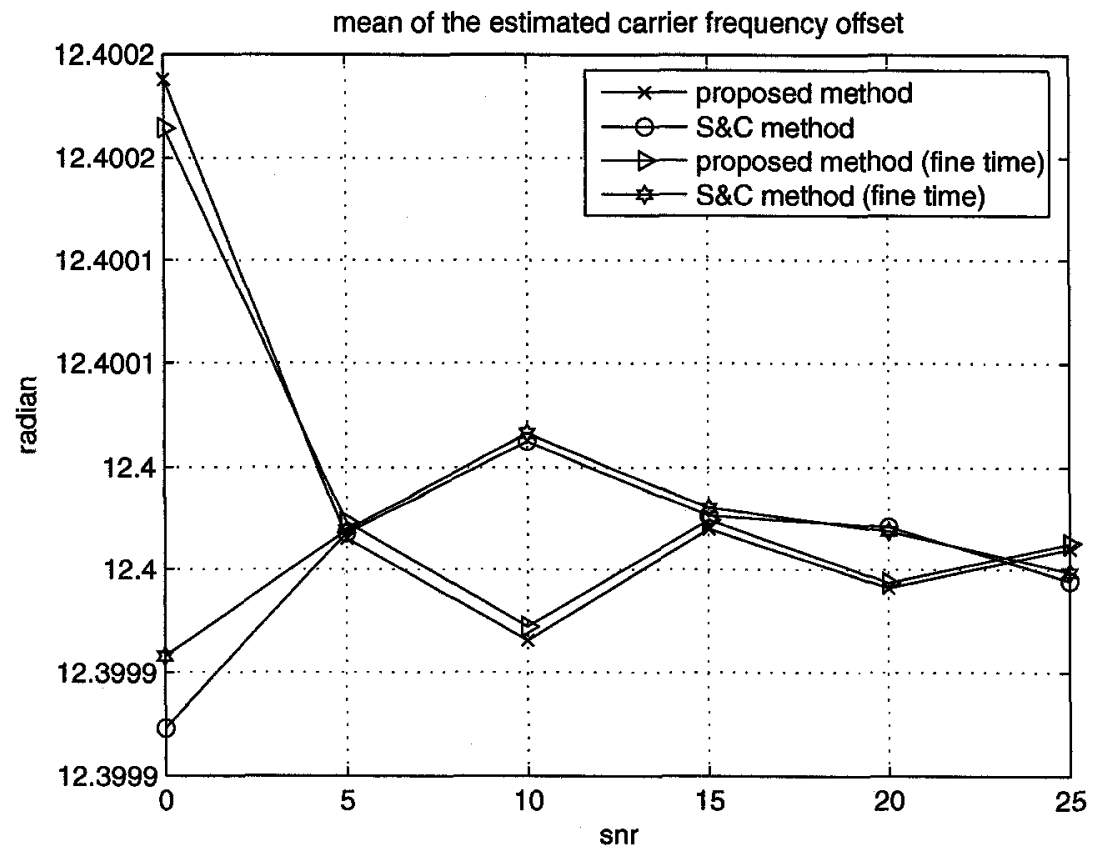

(a) Mean of the CFO

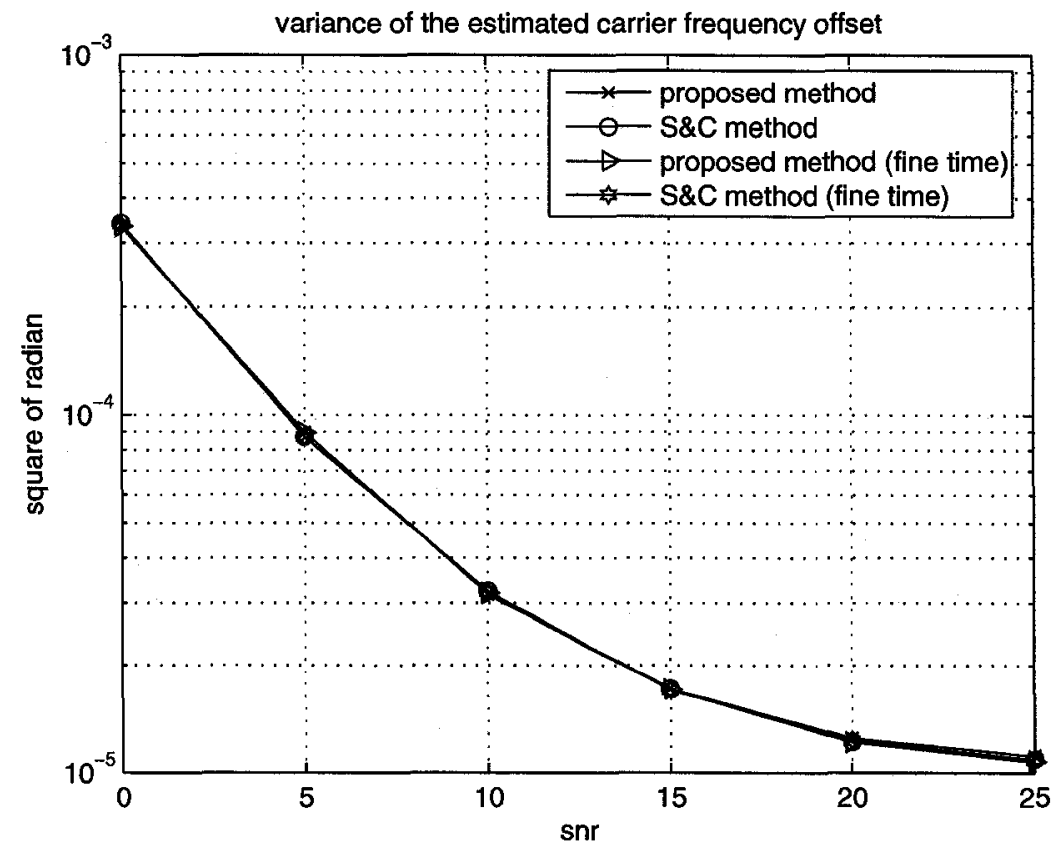

(b) Variance of the CFO

Fig. 5.21 Performance of the CFO estimator in the time-variant Rayleigh fading channel, $v=250 \mathrm{~km} / \mathrm{hr}, f_{m}=1157.4 \mathrm{H}_{z}$ 


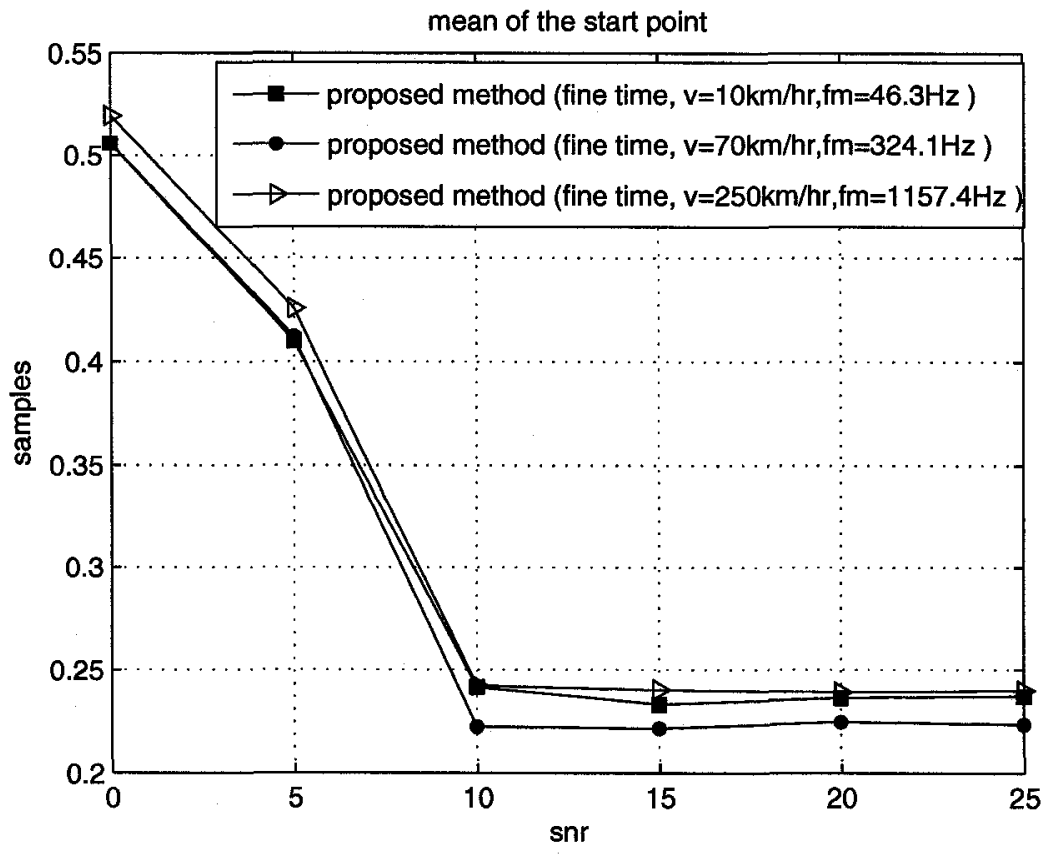

(a) Mean of the start point

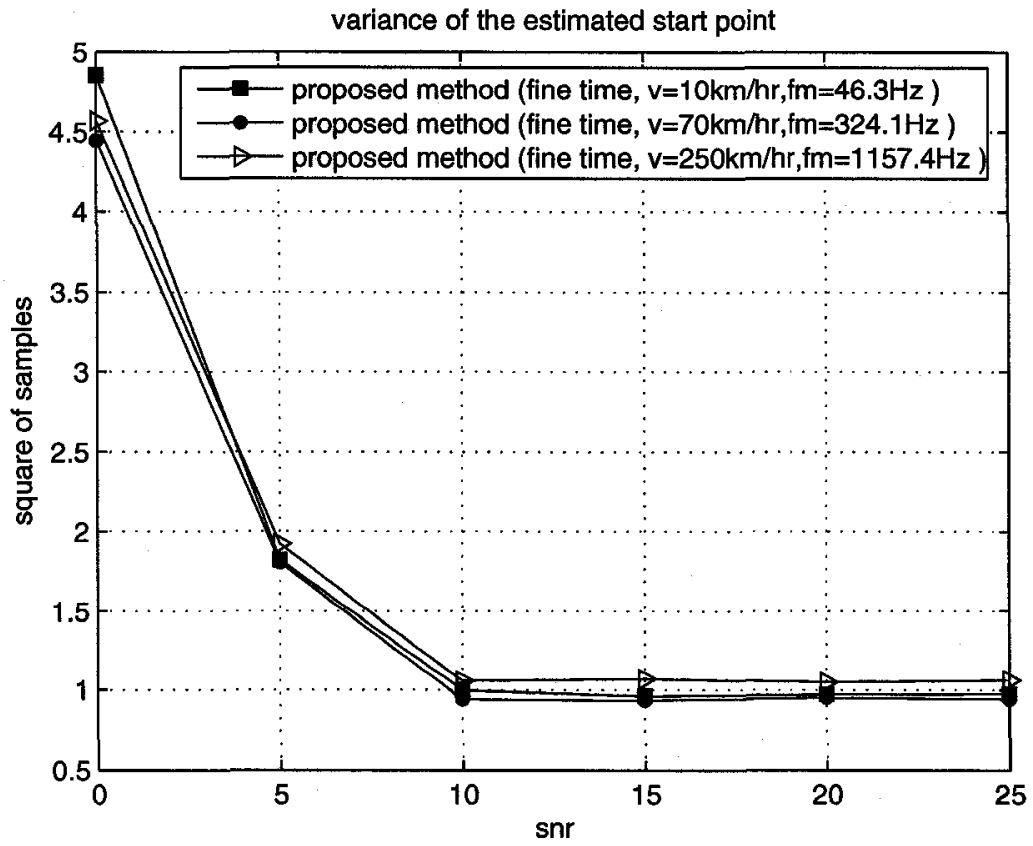

(b) Variance of the start point

Fig. 5.22 Performance of the timing estimator in the time-variant Rayleigh fading channel for various maximum Doppler frequencies 


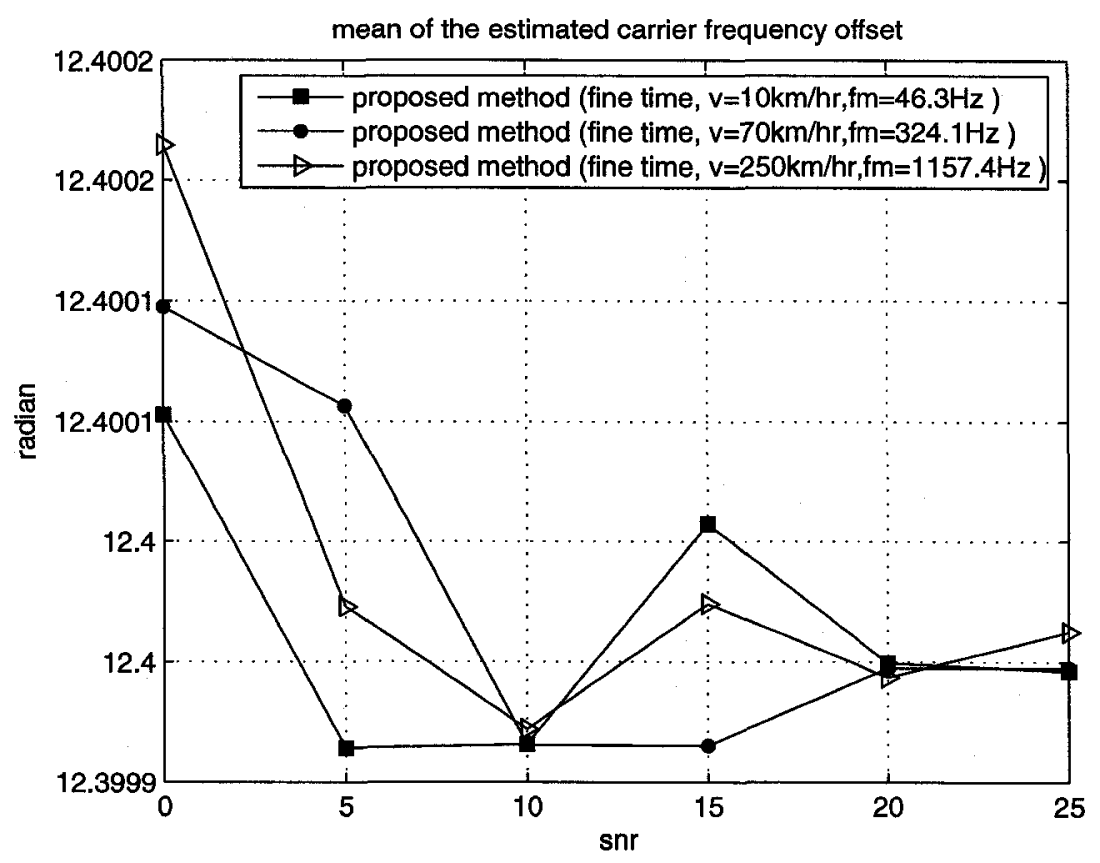

(a) Mean of the CFO

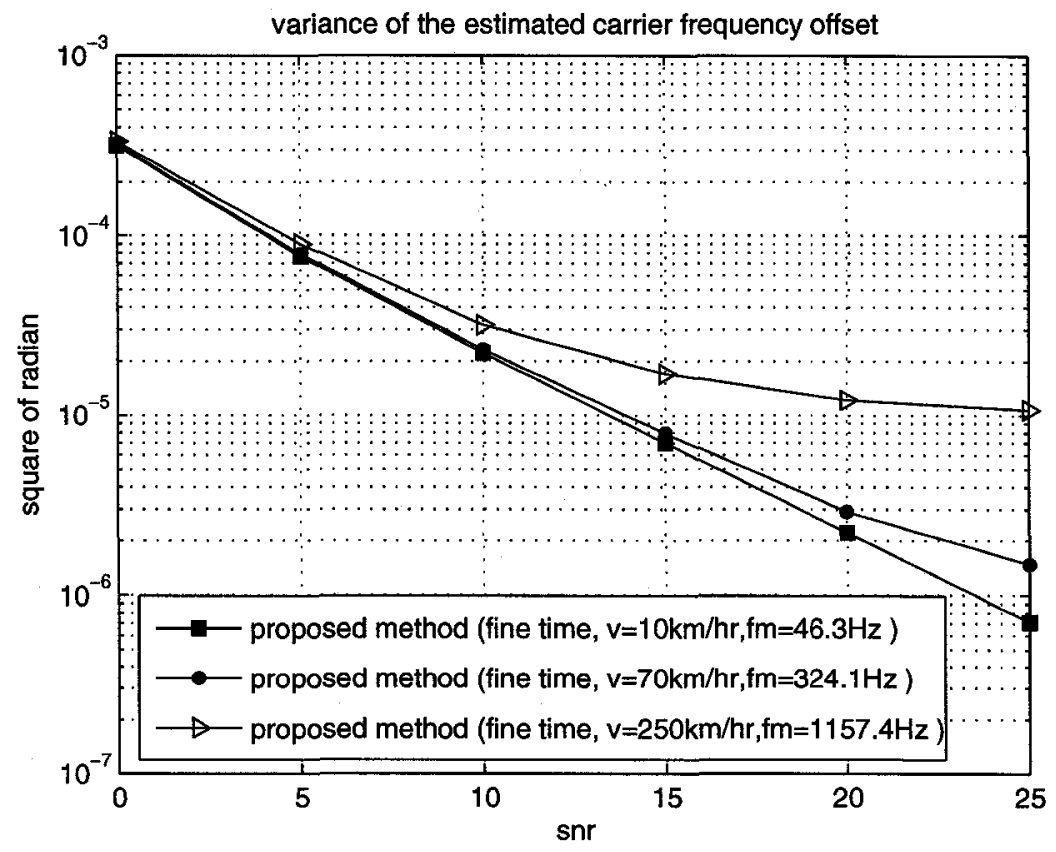

(b) Variance of the CFO

Fig. 5.23 Comparing the performance of the CFO estimator in the time-variant Rayleigh fading channel for various maximum Doppler frequencies 
methods for each simulated SNR.

Therefore, it is seen that the proposed method is quite robust to channel time variations.

\subsection{Simulation results for MIMO-OFDM systems}

\subsubsection{Simulation results in the time-invariant Rayleigh fading channel for MIMO-OFDM systems}

Fig. 5.24 and Fig. 5.25 present the simulation results in terms of the means and variances of the symbol timing and CFO estimators for SC's and proposed methods in the time-invariant Rayleigh fading channel using two transmitter antennas and two receiver antennas.

Fig. 5.24 shows the performance of the coarse and fine timing estimators for different methods using different combining methods in the time-invariant Rayleigh fading channel for a $2 \times 2$ antenna array. Fig. 5.24(a) shows that the mean value of the estimated start point is almost the same using EGC or MRC with a slight advantage for MRC. It also shows that the mean value of the proposed coarse timing estimator is quite close to the exact start point, whereas the SC's coarse timing estimator one shifts to the left of it. The mean values of the proposed and SC fine timing estimators slightly shift to the right of the exact start point because the first tap might be in deep fade and smaller than the threshold in some simulation runs. Fig. 5.24(b) shows that the proposed fine timing estimator has better performance than SC's fine timing estimator at low SNR due to the FlP-based preamble. The proposed coarse timing estimator has a smaller variance than the proposed fine timing estimator when $S N R \geq 15 \mathrm{~dB}$. When $S N R \geq 10 \mathrm{~dB}$, the proposed fine timing estimator has almost the same variance. This error floor is caused by the probability of false alarm and 
missing detection of the fine timing estimator. It depends on the threshold setting and tap fading. Since the correlation function used for the proposed coarse timing estimator collects all tap signal powers, the accuracy of the proposed coarse timing estimator increases with SNR. The proposed coarse timing estimator variance using MRC is slightly lower than the one using EGC.

Fig. 5.25 shows the performance of the CFO estimators for different methods using different combining methods in the time-invariant Rayleigh fading channel for a $2 \times 2$ antenna array. It shows that the proposed CFO estimator has a similar variance as SC's CFO estimator one at low SNR and slightly bigger at high SNR. Performance using EGC or MRC is almost the same. The integer part of the CFO is estimated correctly for 10,000 runs by SC's and proposed methods for each simulated SNR.

In Fig. 5.26, we compare the performance of the proposed coarse timing estimator using different number of transmitter and receiver antennas with EGC. Due to the spatial diversity, the mean value of the proposed coarse timing estimator is closer to the exact start point and the variance is getting lower when the antenna number is increased. The receiver diversity gives more gain than transmitter diversity.

In Fig. 5.27, we compare the performance of the proposed fine timing estimator using different number of transmitter and receiver antennas with EGC. Since the total transmitted power is fixed, increasing the transmitter antennas means decreasing the transmitted power on each transmitter antenna. From (4.41), increasing the number of the transmitter antennas will decrease the mean value of $\psi_{l, n}$ and the probability of false alarm and missing detection of fine timing estimator will increase (appendix B). The performance of the proposed fine timing estimator depends on both the performance of the proposed coarse timing estimator and the probability of false alarm and missing detection of the fine timing estimator. At low SNR, the performance of the proposed fine timing estimator is dominated by the performance 
of the proposed coarse timing estimator (in the searching range or not) and $2 \times 3$ has the lowest variance when $S N R=0 \mathrm{~dB}$. At high $\mathrm{SNR}$, the performance of the proposed fine timing estimator is dominated by the probability of false alarm and missing detection of the fine timing estimator and $1 \times 2$ has the lowest variance when $S N R \geq 5 \mathrm{~dB}$.

In Fig. 5.28, we compare the performance of the proposed CFO estimators using different number of transmitter and receiver antennas with EGC. Fig. 5.28 shows that increasing the number of transmitter antenna or receiver antenna improves the performance of the proposed CFO estimator. The receiver diversity gives more gain for the CFO estimation than the transmitter diversity.

Summarizing, it is seen that the performance when using EGC or MRC is almost the same with a slight improvement using MRC. For MIMO-OFDM systems, it is preferable to use the proposed fine timing estimator in low SNR scenario and the proposed coarse timing estimator in high SNR scenario to achieve the best performance. Overall, the proposed method works well in the time-invariant Rayleigh fading channel for MIMO-OFDM systems. 


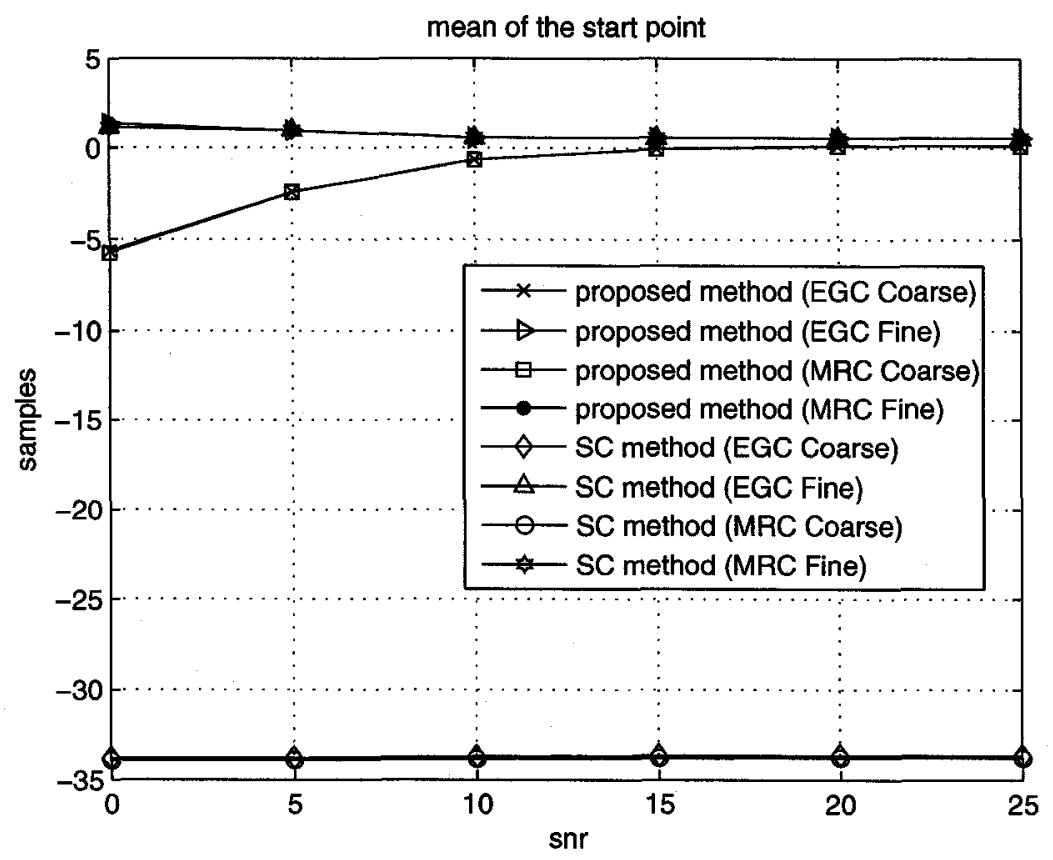

(a) Mean of the start point

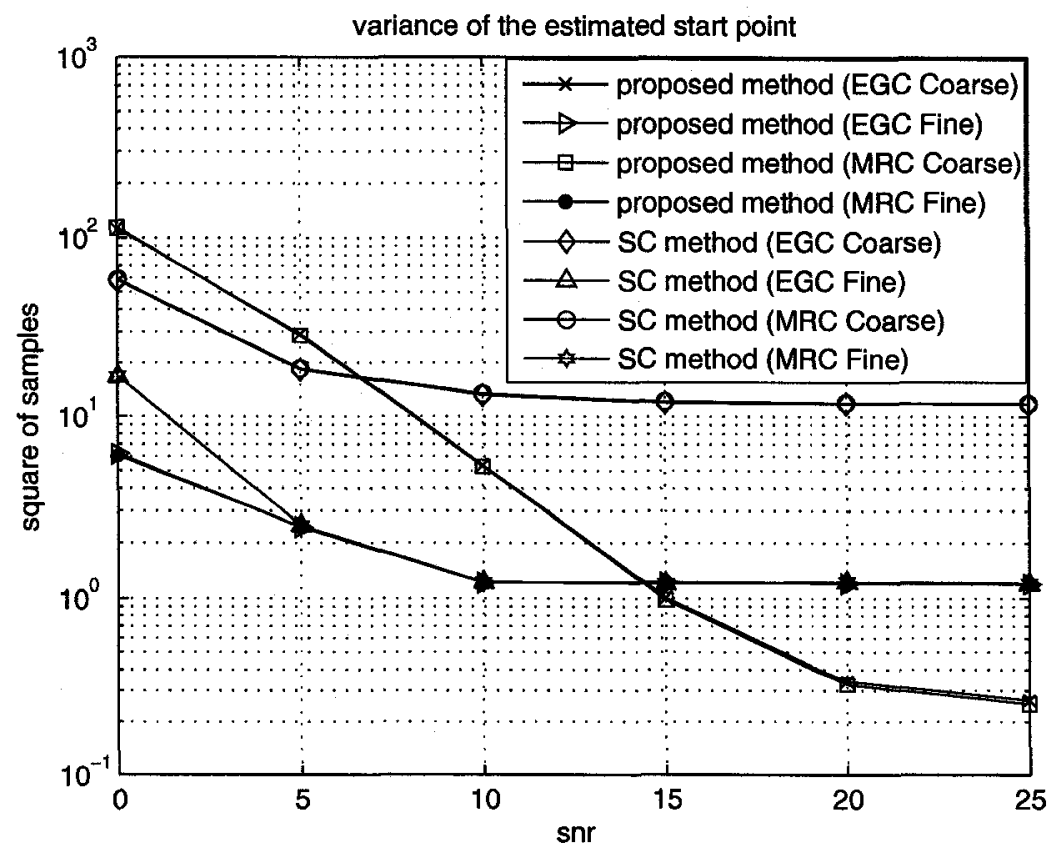

(b) Variance of the start point

Fig. 5.24 Performance of the timing estimator in the time-invariant Rayleigh fading channel, $Q=2, L=2$ 


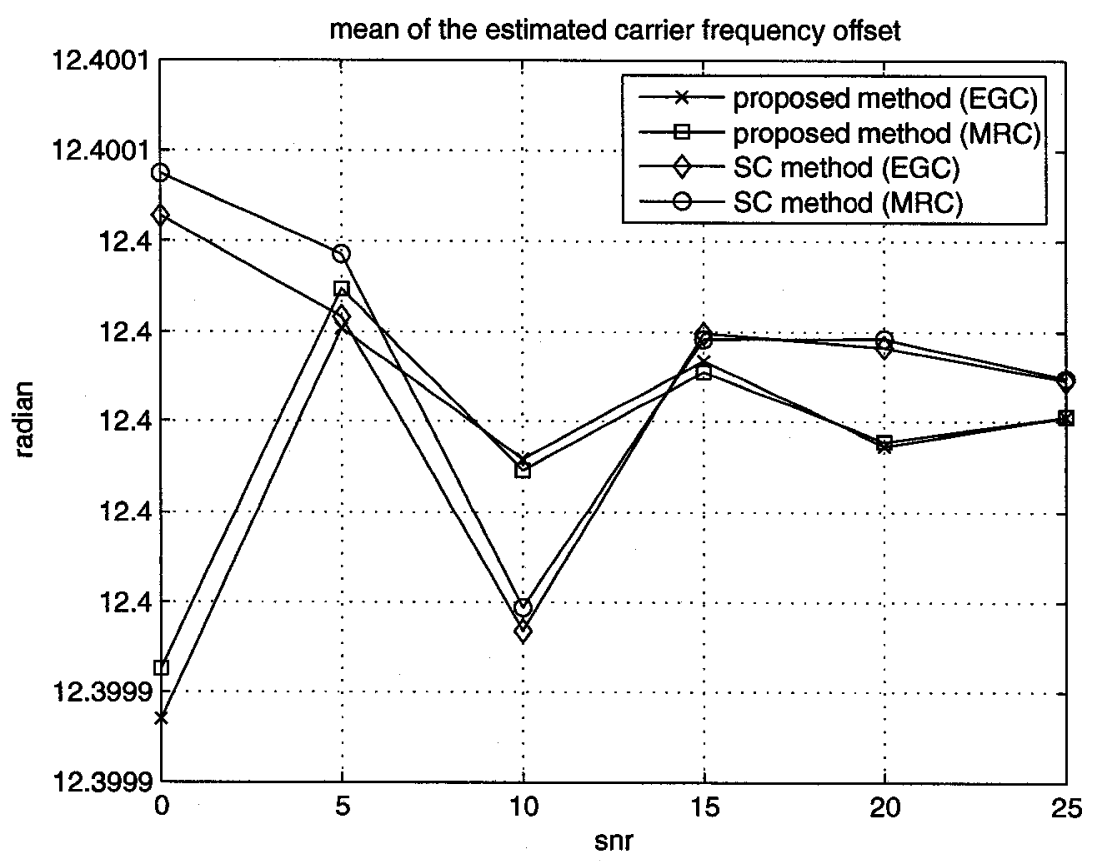

(a) Mean of the CFO

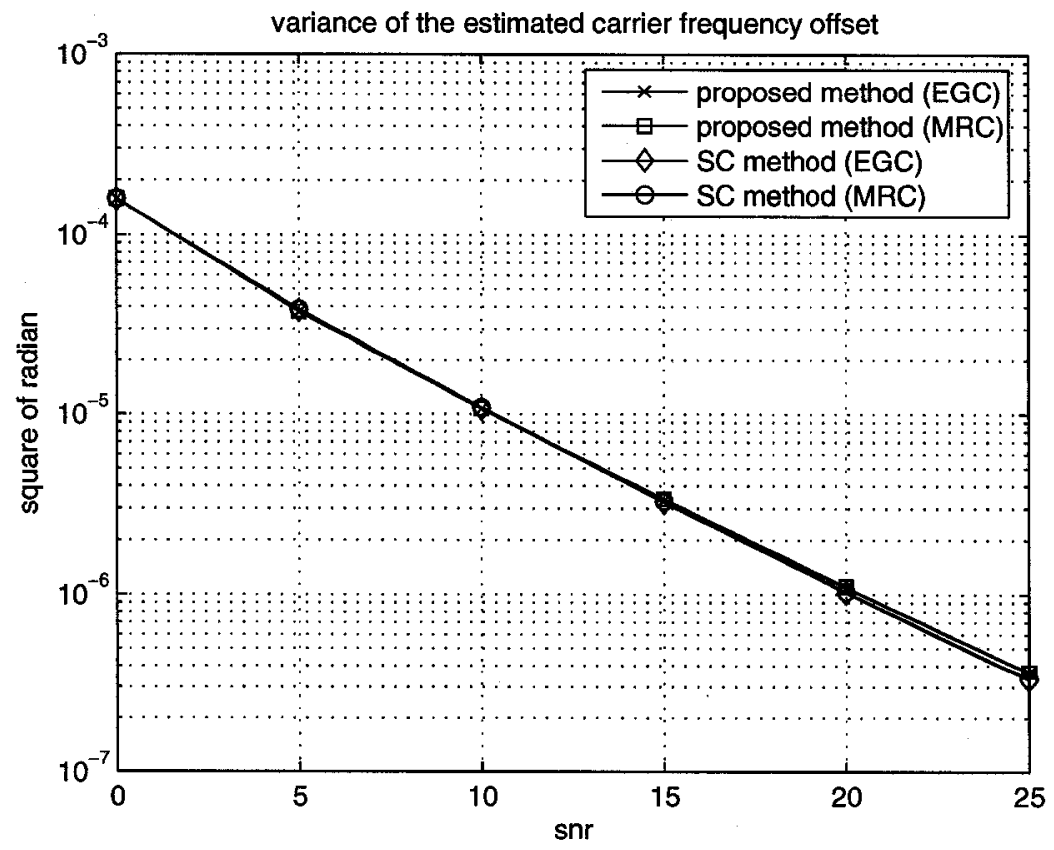

(b) Variance of the CFO

Fig. 5.25 Performance of the CFO estimator in the time-invariant Rayleigh fading channel, $Q=2, L=2$ 


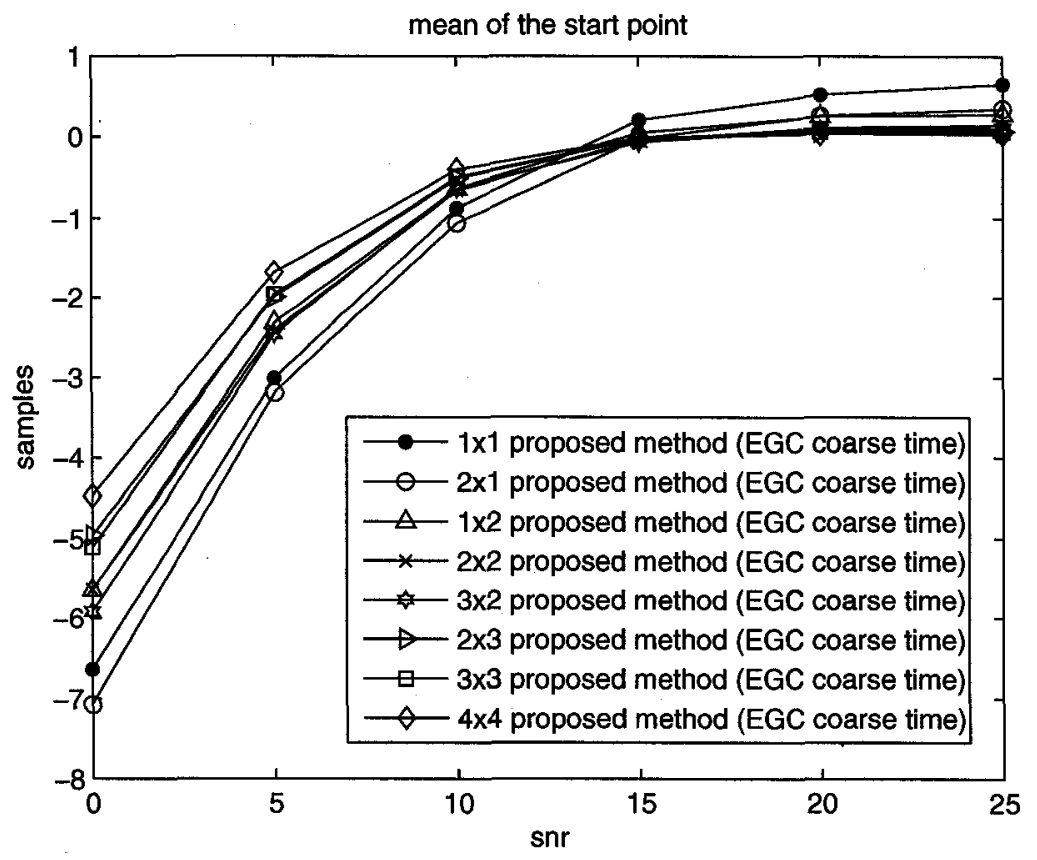

(a) Mean of the start point

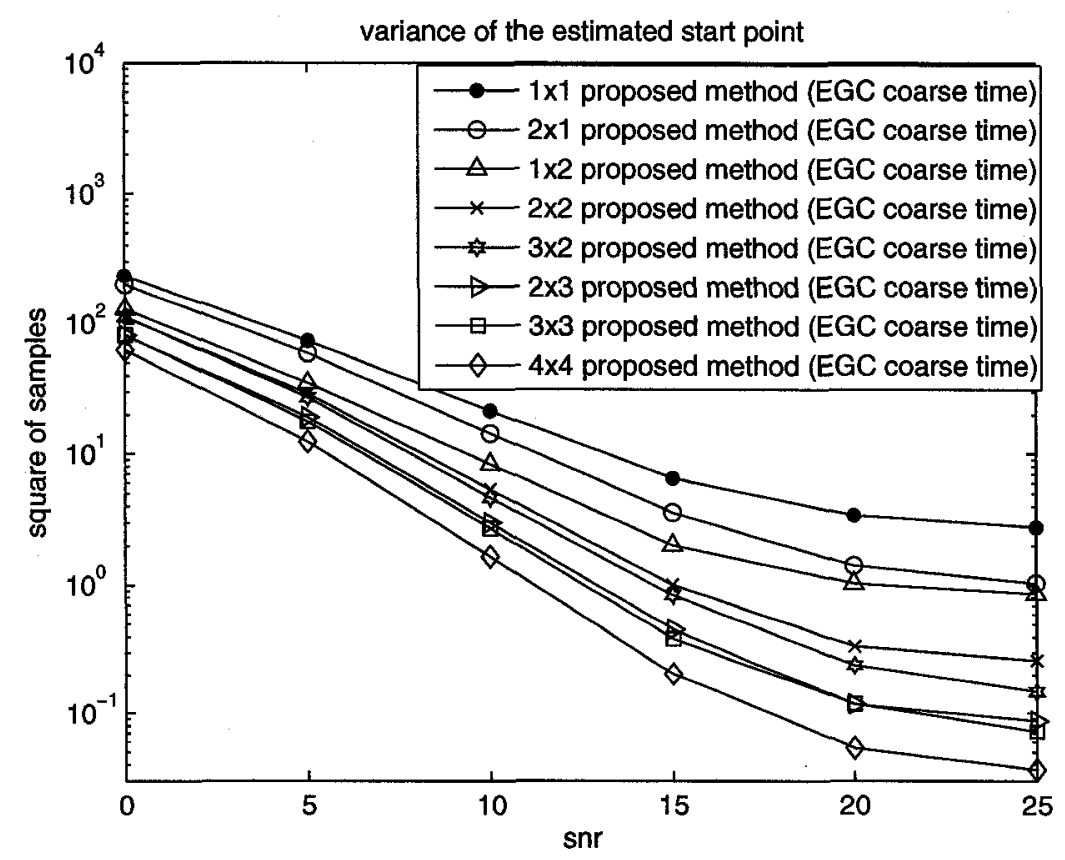

(b) Variance of the start point

Fig. 5.26 Performance of the proposed coarse timing estimator in the timeinvariant Rayleigh fading channel using different number of antennas 


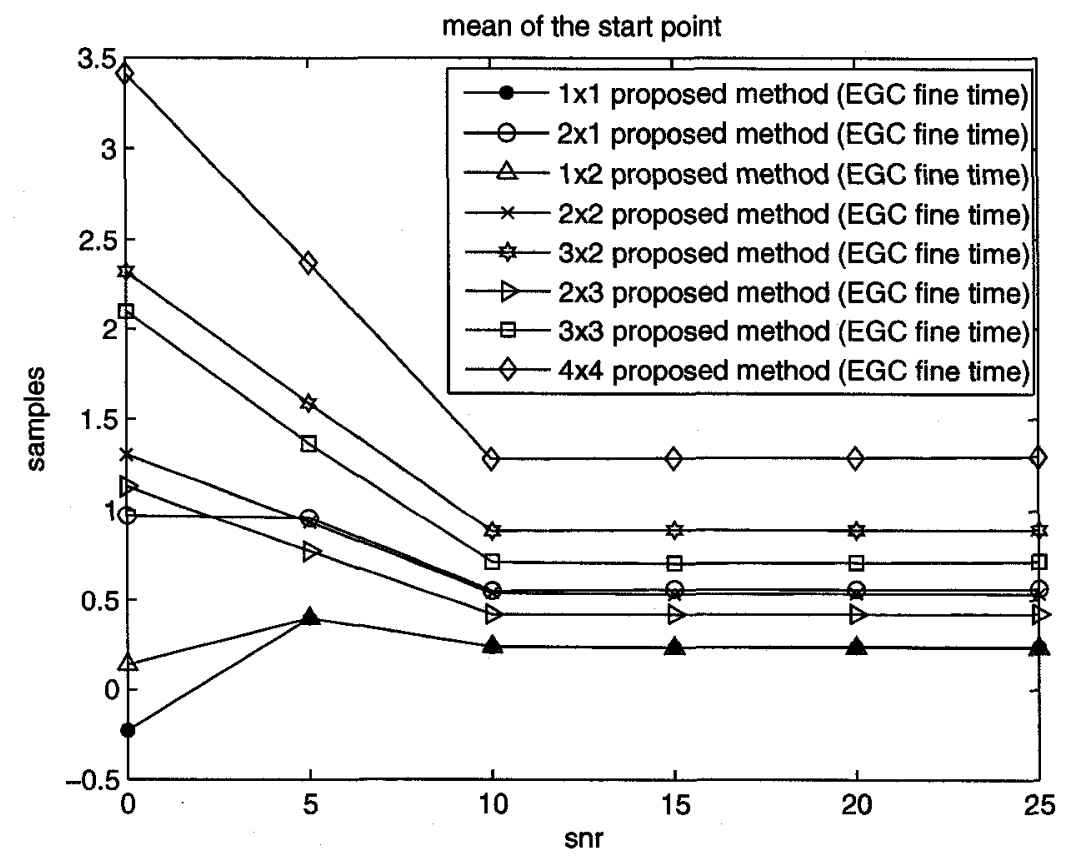

(a) Mean of the start point

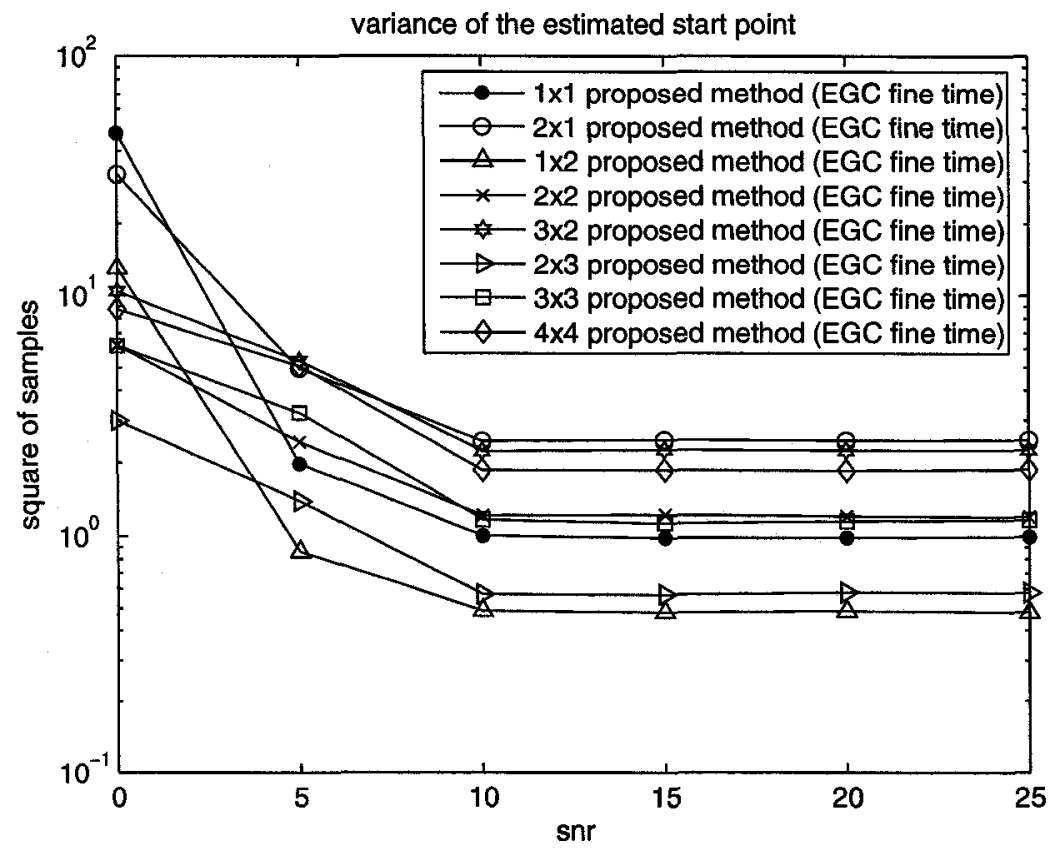

(b) Variance of the start point

Fig. 5.27 Performance of the proposed fine timing estimator in the time-invariant Rayleigh fading channel using different number of antennas 


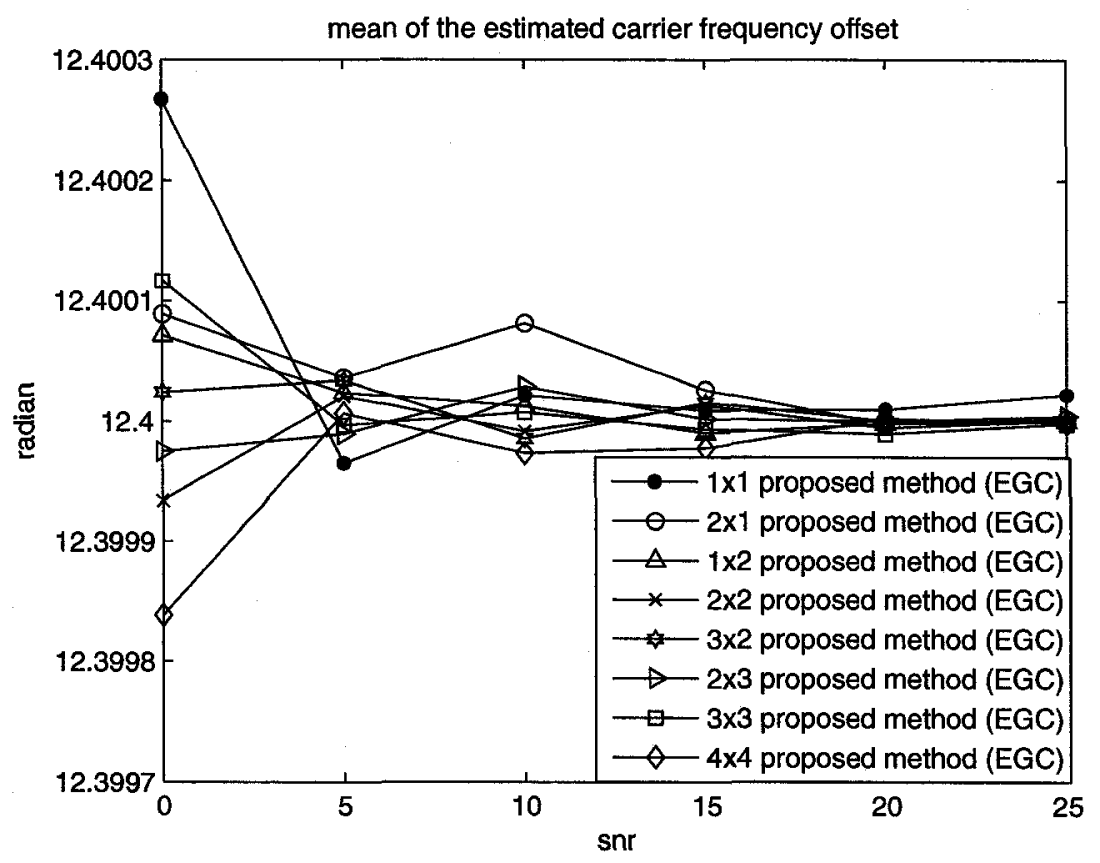

(a) Mean of the CFO

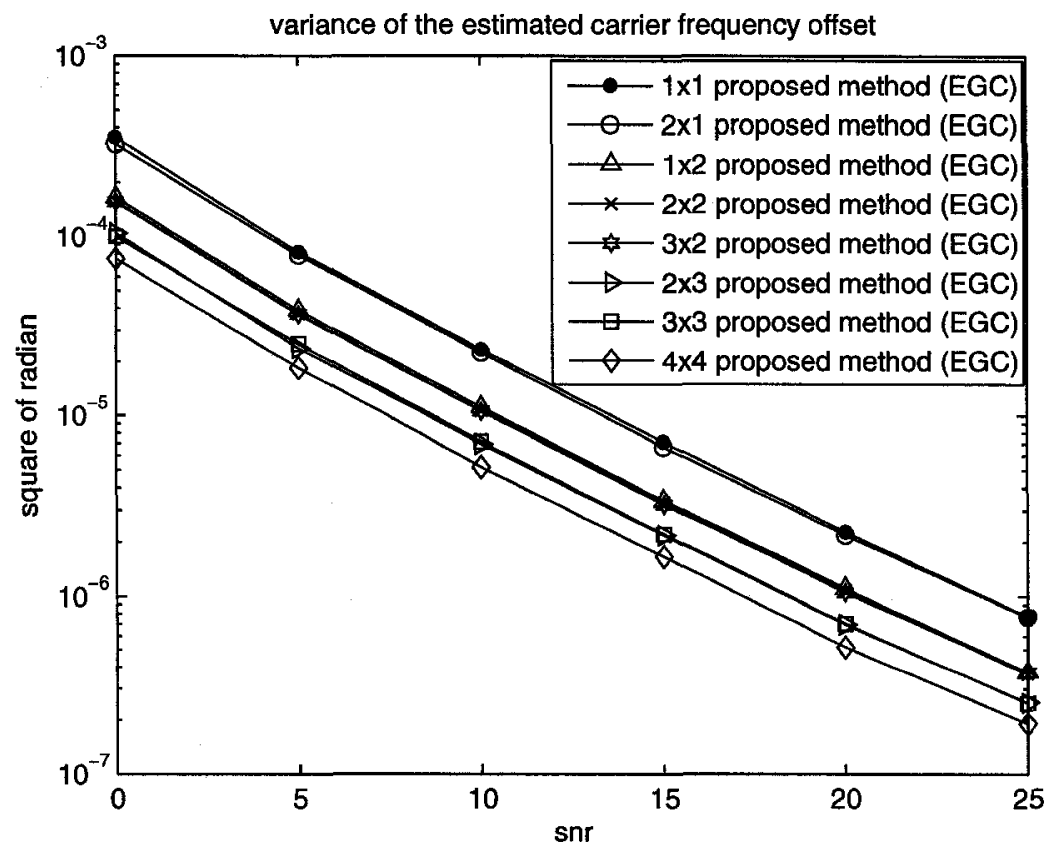

(b) Variance of the CFO

Fig. 5.28 Performance of the proposed CFO estimator in the time-invariant Rayleigh fading channel using different number of antennas 


\subsubsection{Simulation results in the time-variant Rayleigh fading channel for MIMO-OFDM systems}

Figs. 5.29-5.34 present the simulation results in terms of the means and variances of the symbol timing and CFO estimators for SC's and proposed methods in the time-variant Rayleigh fading channel using two transmitter antennas and two receiver antennas with different maximum Doppler frequencies (corresponding to low, moderated and high vehicle speed).

For each maximum Doppler frequency, the proposed fine timing estimator has the best performance. The variance of the proposed CFO estimator is similar to SC's CFO estimator one at low SNR and slightly bigger at high SNR. Performance when the fine time point or the coarse time point is used to estimate CFO are almost the same.

In Fig. 5.35 and Fig. 5.36, we compare the performance of the proposed timing and CFO estimator with different maximum Doppler frequencies in a $2 \times 2$ MIMO-OFDM system. The timing estimators have almost the same performance when the maximum Doppler frequency changes. When the maximum Doppler frequency increases, the variance of the $\mathrm{CFO}$ estimator also increases. The $\mathrm{CFO}$ estimator is more sensitive to the rate of the channel change. Overall, it is seen that the proposed method is robust to channel time variations for MIMO-OFDM systems as well. 


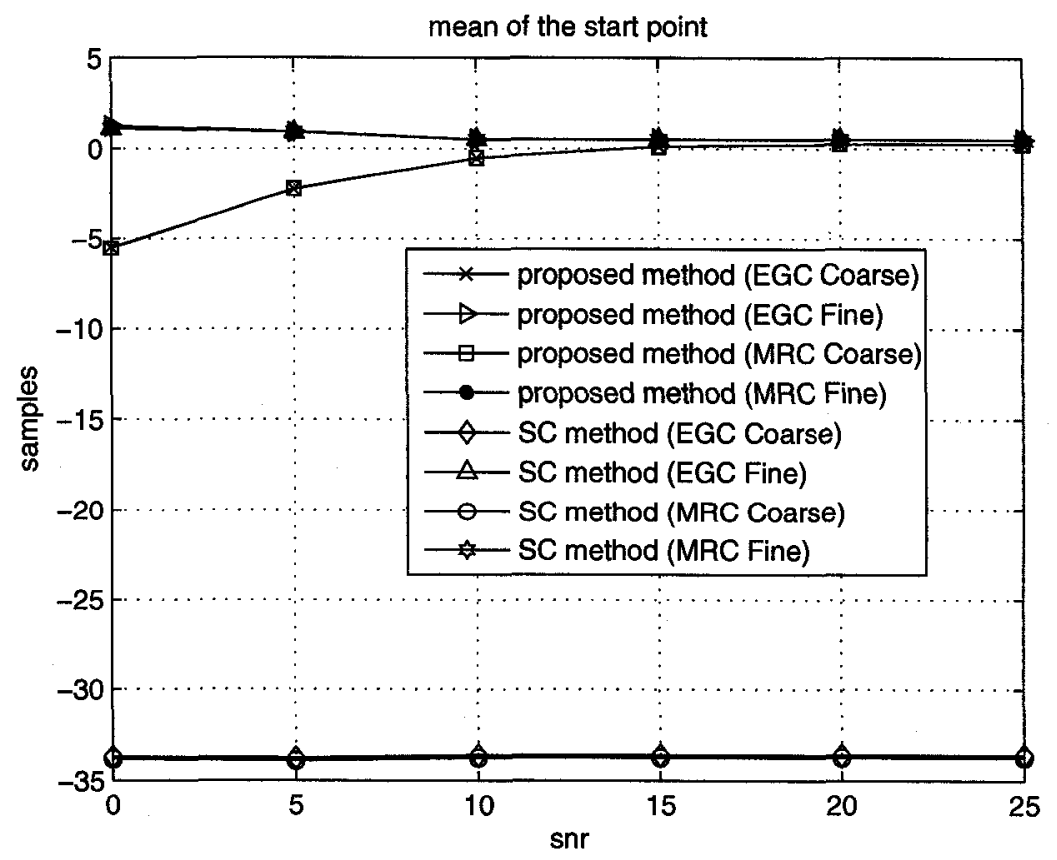

(a) Mean of the start point

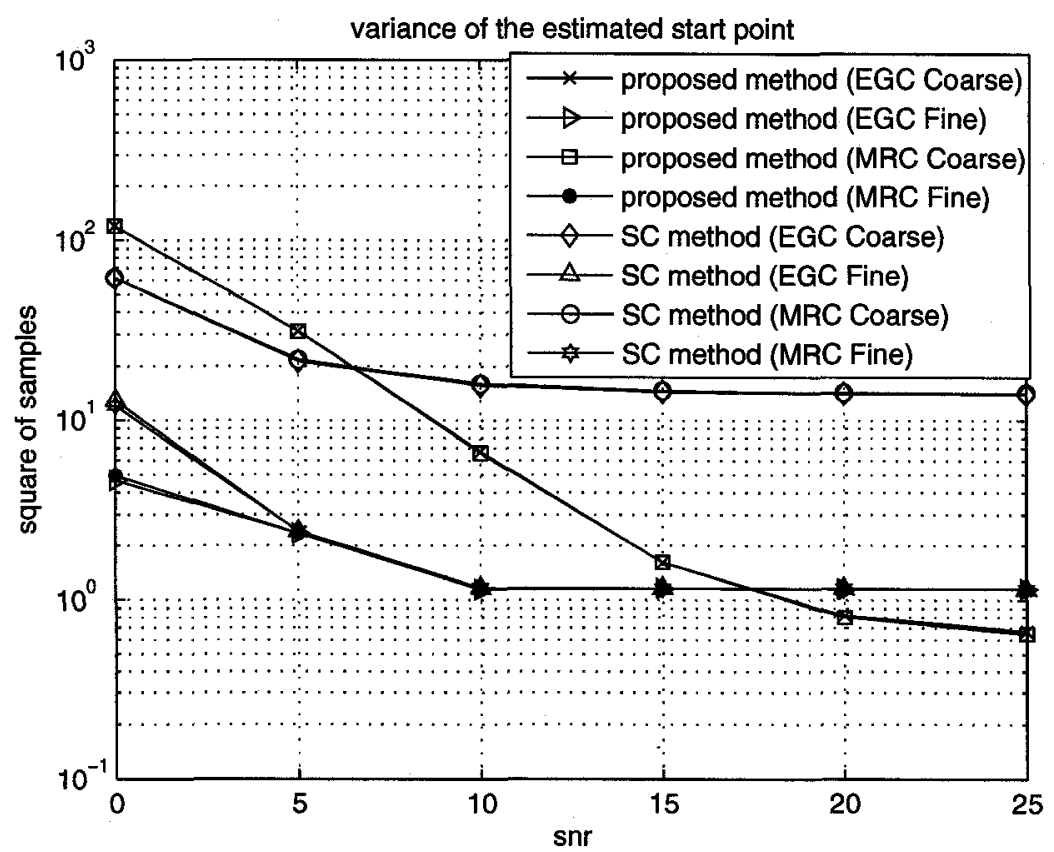

(b) Variance of the start point

Fig. 5.29 Performance of the timing estimator in the time-variant Rayleigh fading channel, $Q=2, L=2, v=10 \mathrm{~km} / \mathrm{hr}, f_{m}=46.3 H_{z}$ 


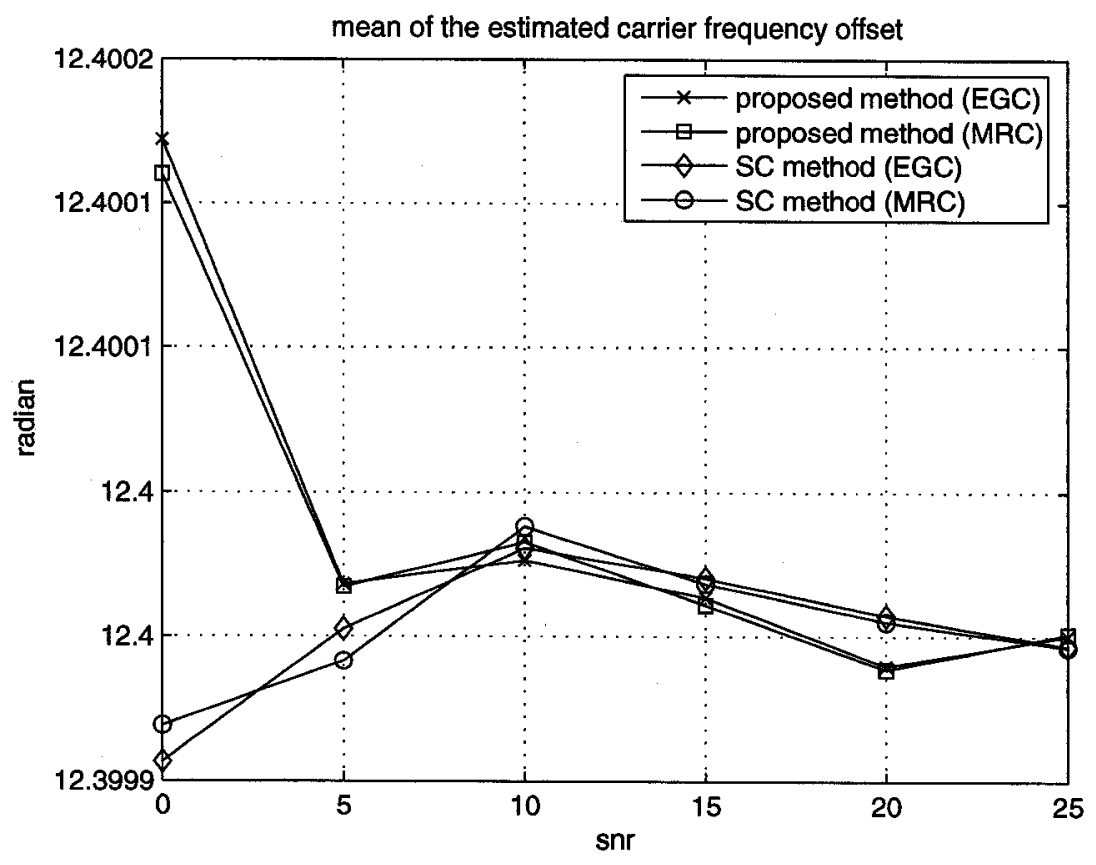

(a) Mean of the CFO

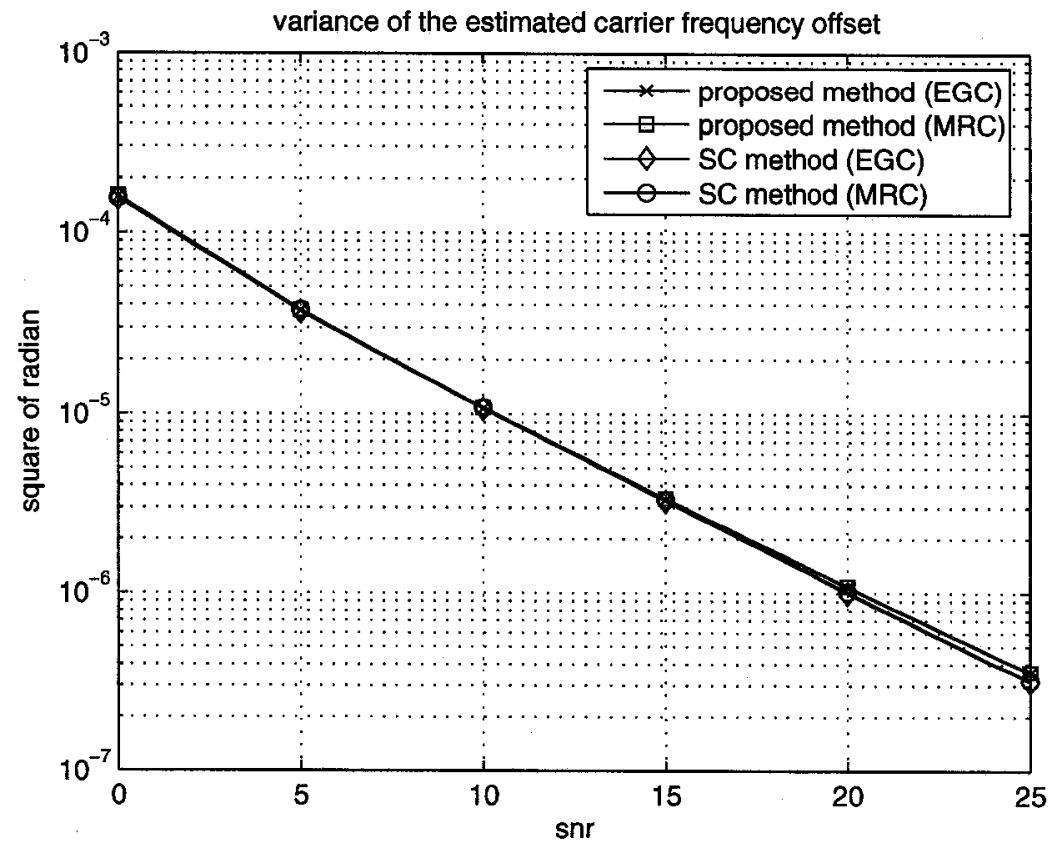

(b) Variance of the CFO

Fig. 5.30 Performance of the CFO estimator in the time-variant Rayleigh fading channel, $Q=2, L=2, v=10 \mathrm{~km} / \mathrm{hr}, f_{m}=46.3 H_{z}$ 


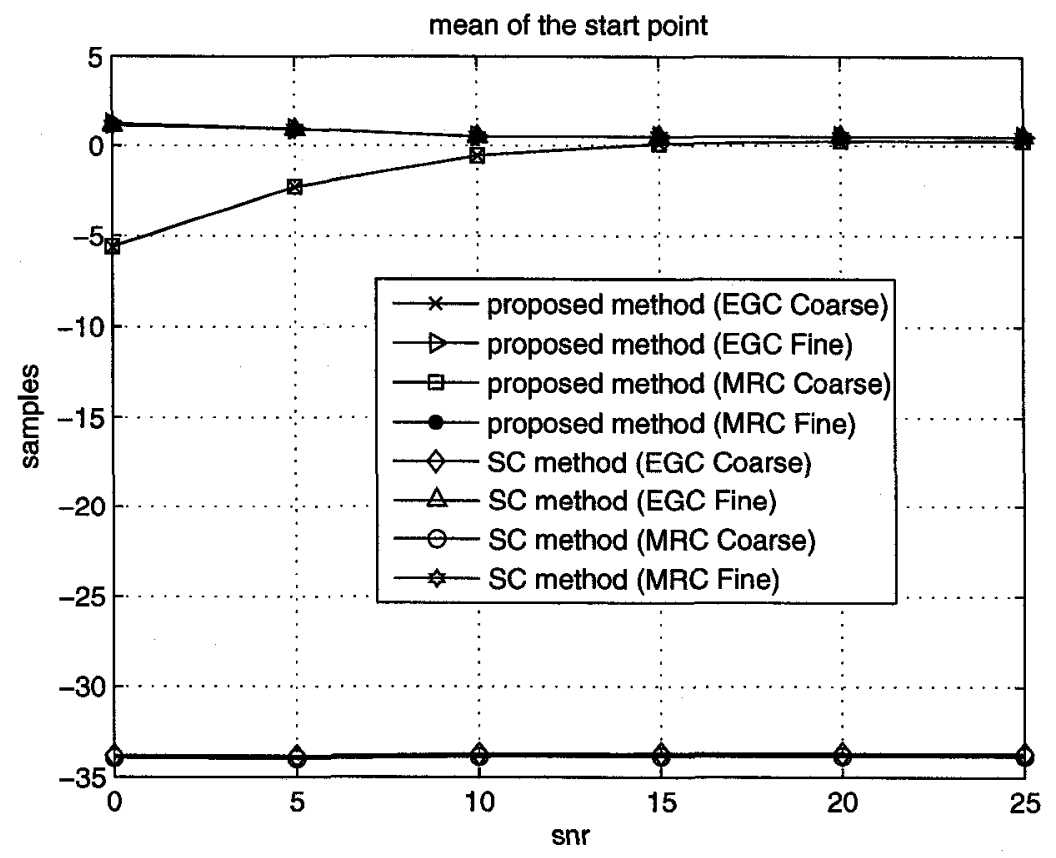

(a) Mean of the start point

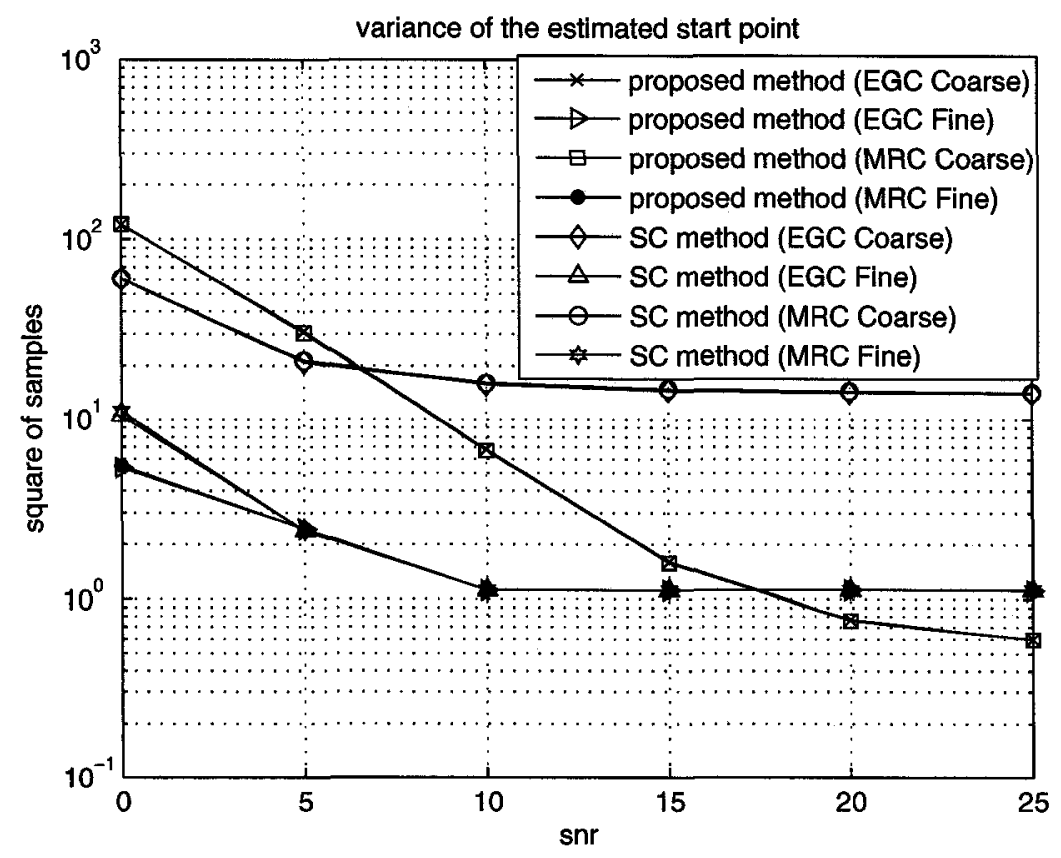

(b) Variance of the start point

Fig. 5.31 Performance of the timing estimator in the time-variant Rayleigh fading channel, $Q=2, L=2, v=70 \mathrm{~km} / \mathrm{hr}, f_{m}=324.1 \mathrm{H}_{z}$ 


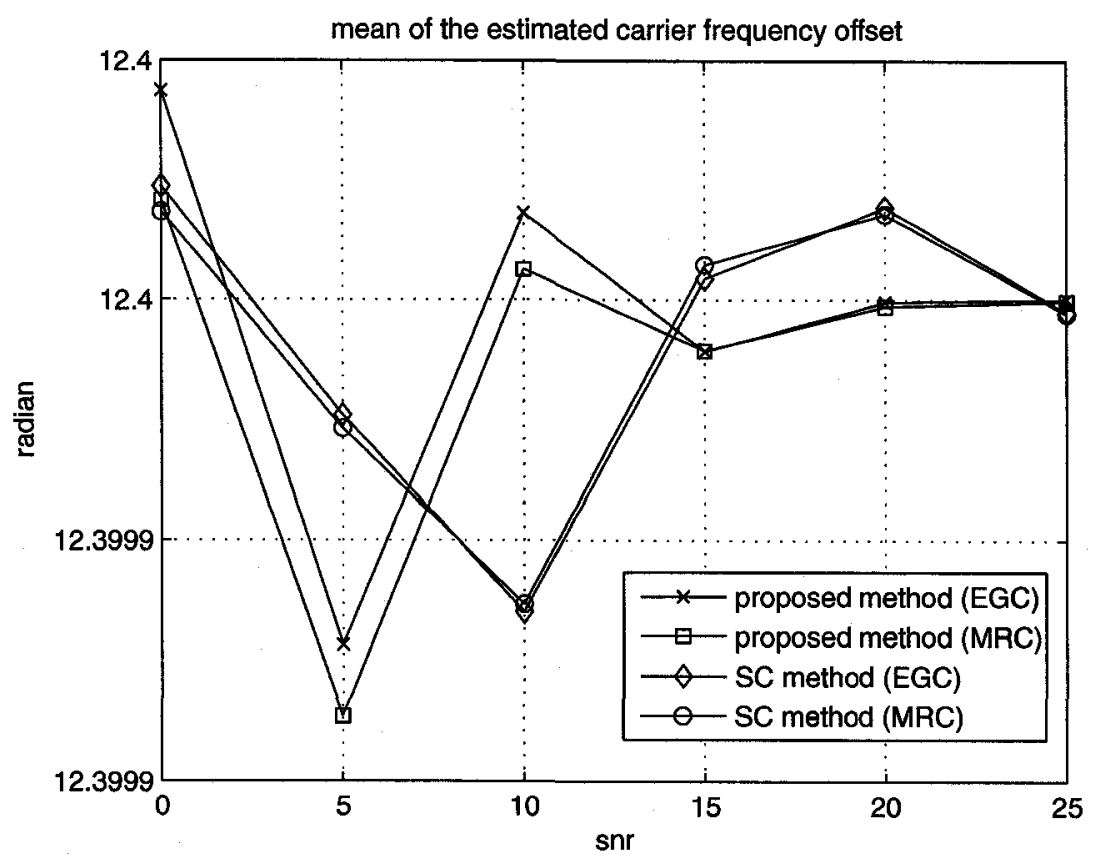

(a) Mean of the CFO

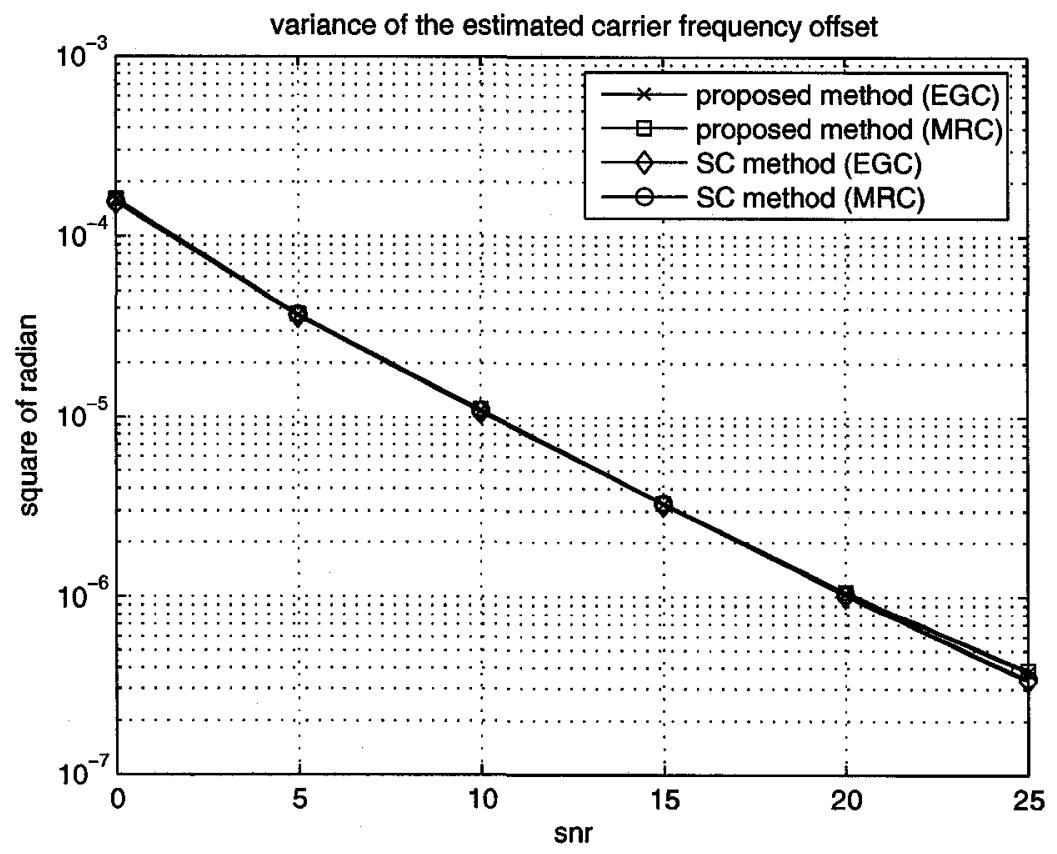

(b) Variance of the CFO

Fig. 5.32 Performance of the CFO estimator in the time-variant Rayleigh fading channel, $Q=2, L=2, v=70 \mathrm{~km} / \mathrm{hr}, f_{m}=324.1 H_{z}$ 


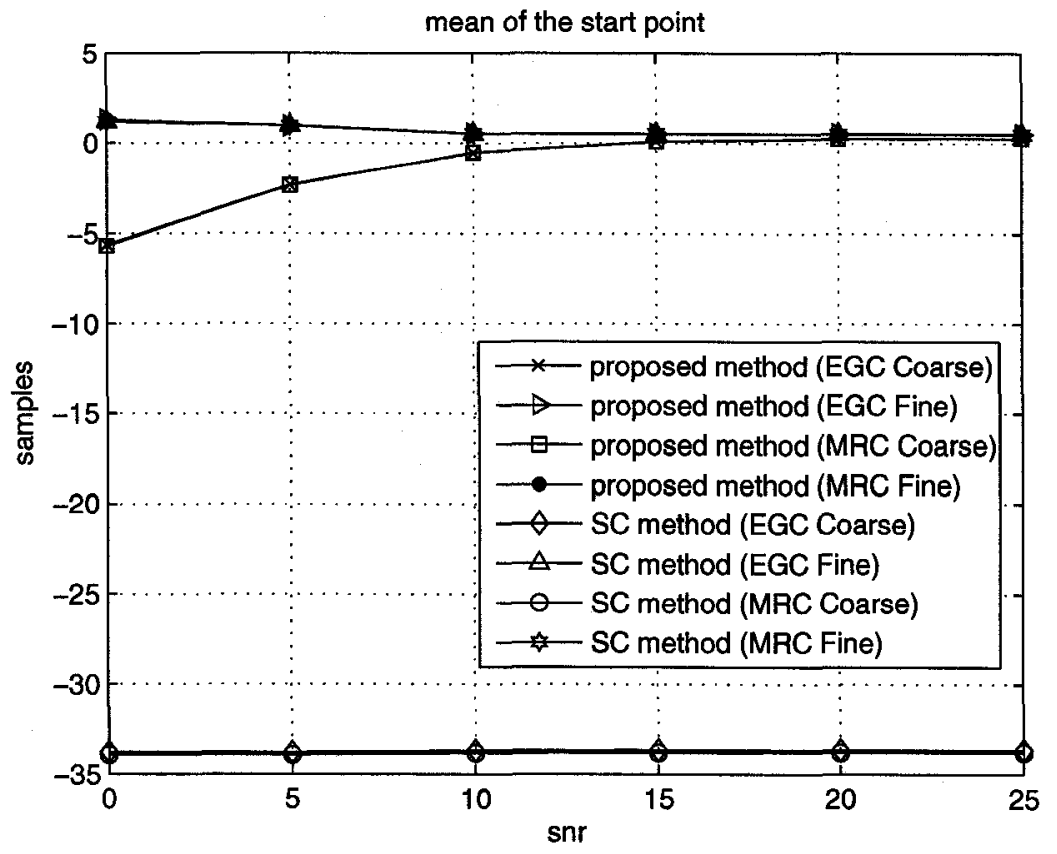

(a) Mean of the start point

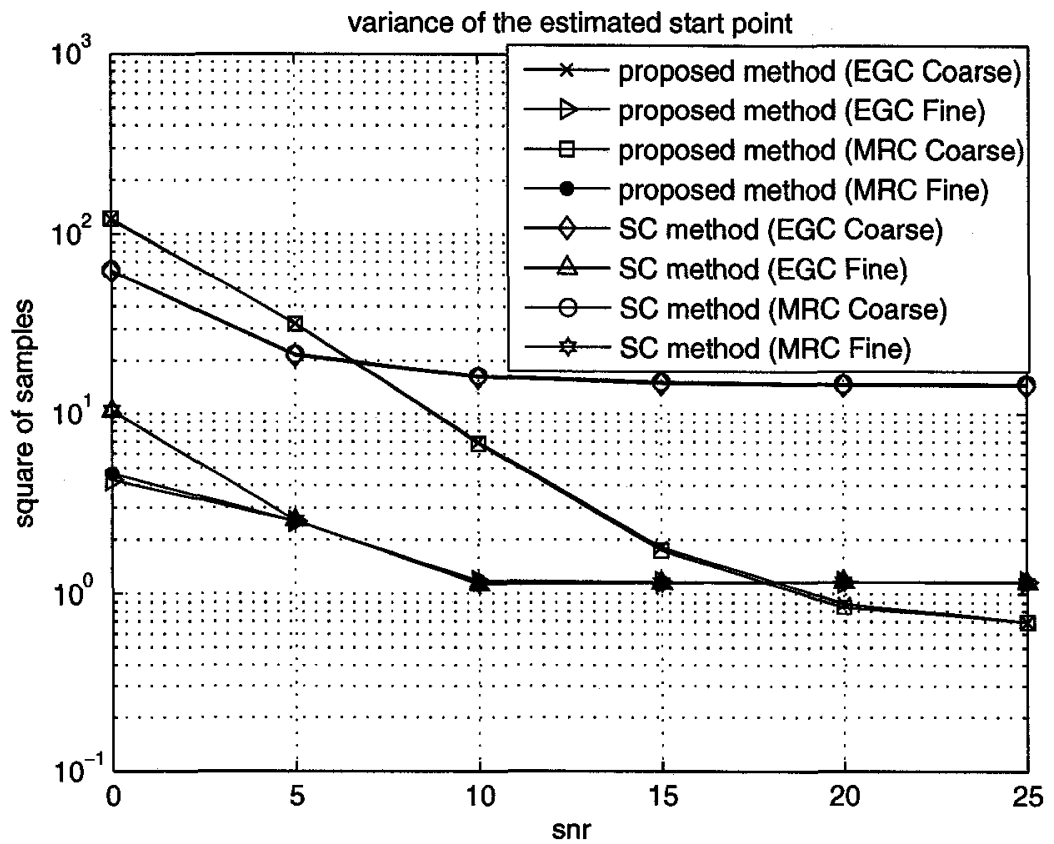

(b) Variance of the start point

Fig. 5.33 Performance of the timing estimator in the time-variant Rayleigh fading channel, $Q=2, L=2, v=250 \mathrm{~km} / \mathrm{hr}, f_{m}=1157.4 H_{z}$ 


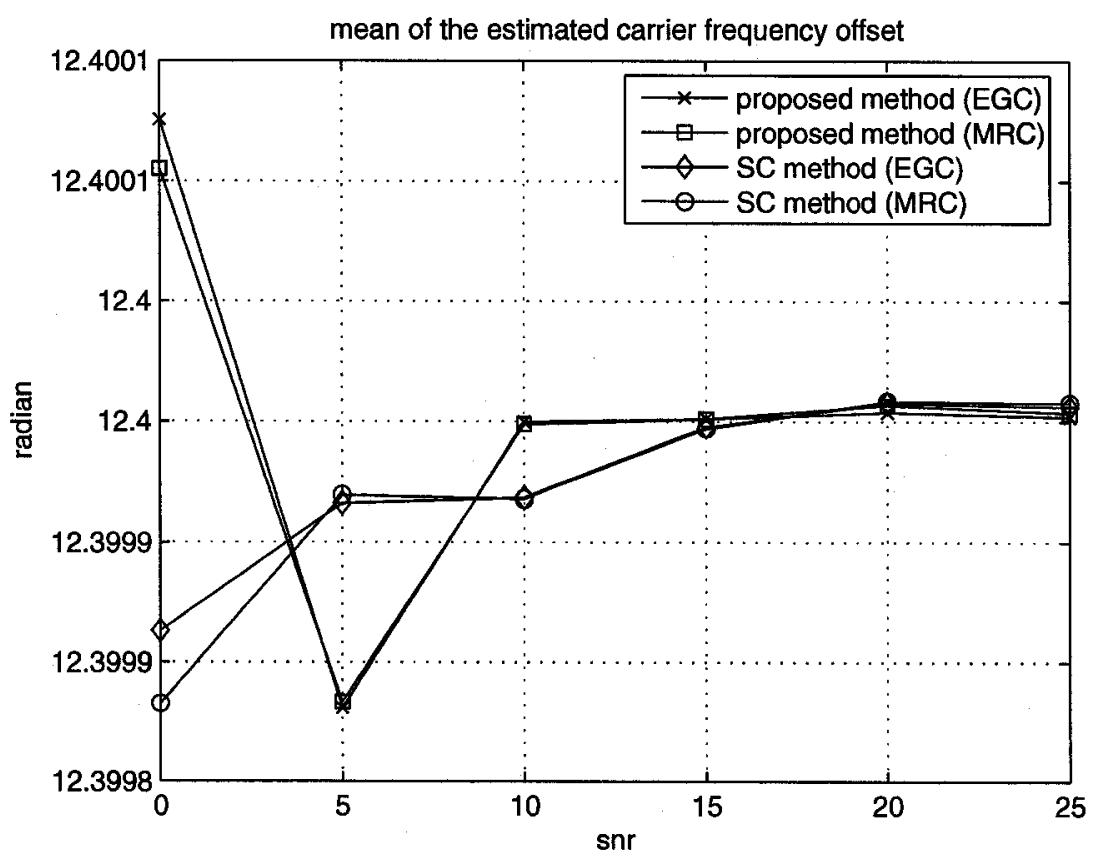

(a) Mean of the CFO

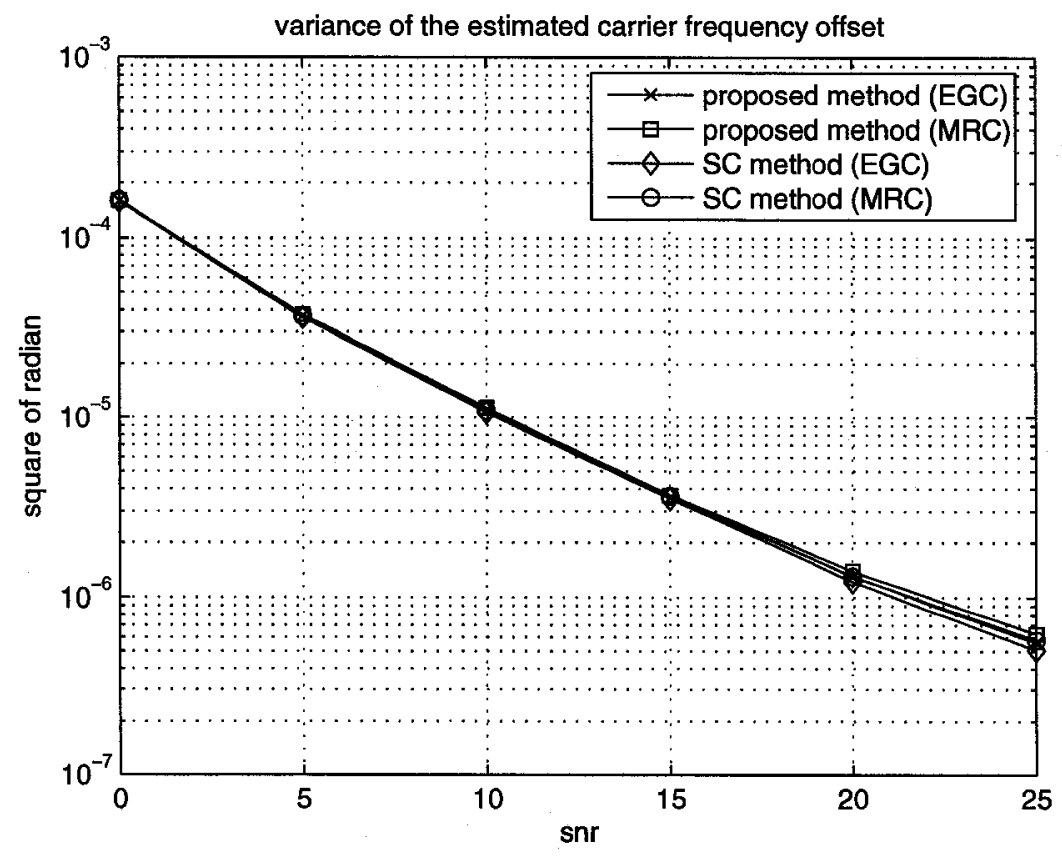

(b) Variance of the CFO

Fig. 5.34 Performance of the CFO estimator in the time-variant Rayleigh fading channel, $Q=2, L=2, v=250 \mathrm{~km} / \mathrm{hr}, f_{m}=1157.4 H_{z}$ 


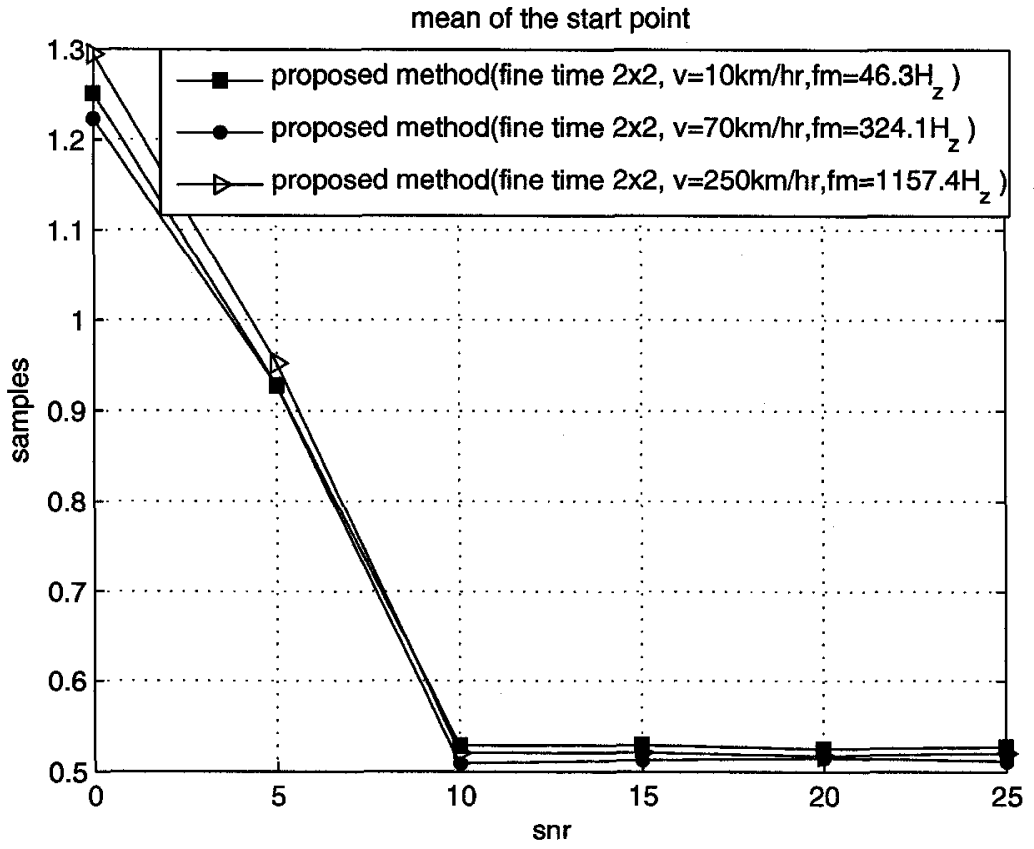

(a) Mean of the start point

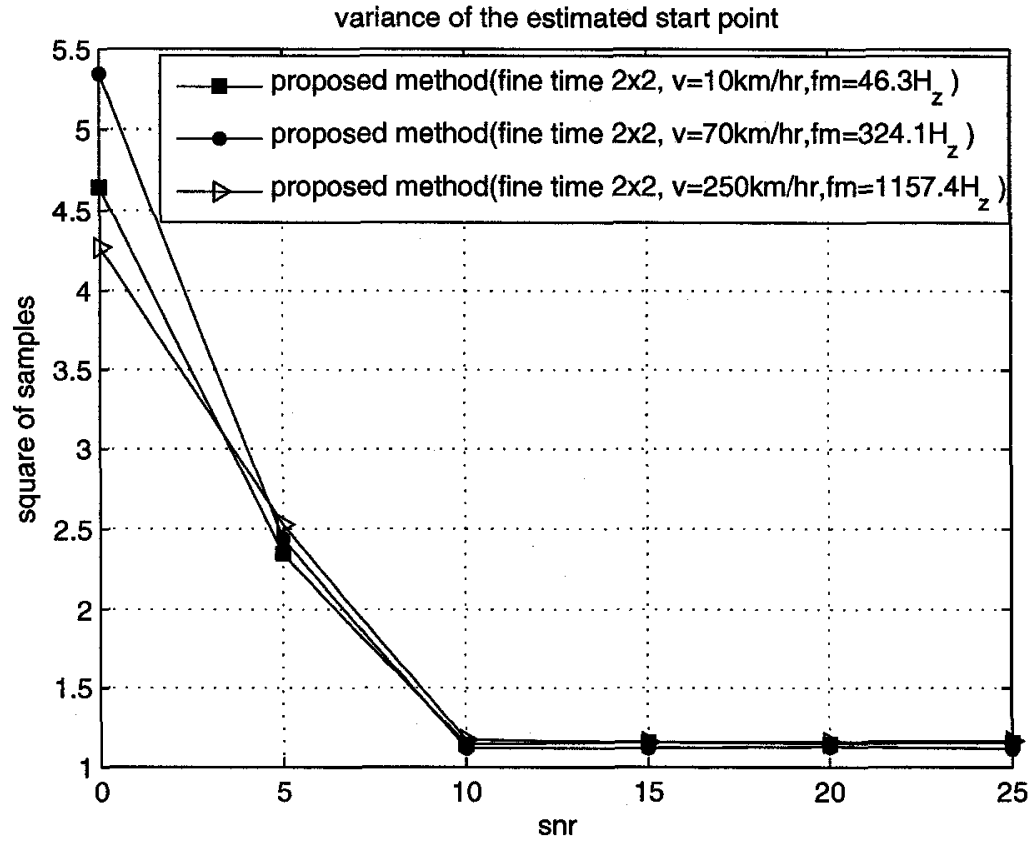

(b) Variance of the start point

Fig. 5.35 Performance of the timing estimator using EGC in the time-variant Rayleigh fading channel for various maximum Doppler frequencies, $Q=2, L=2$ 


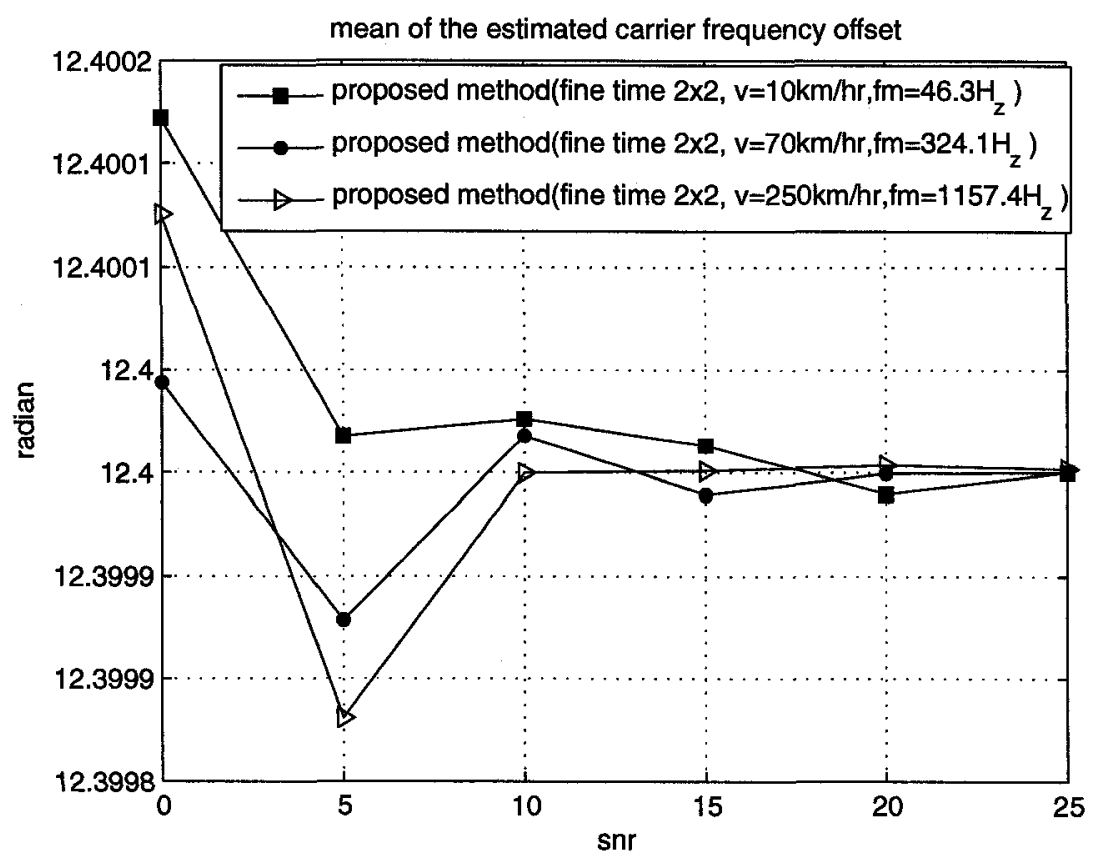

(a) Mean of the CFO

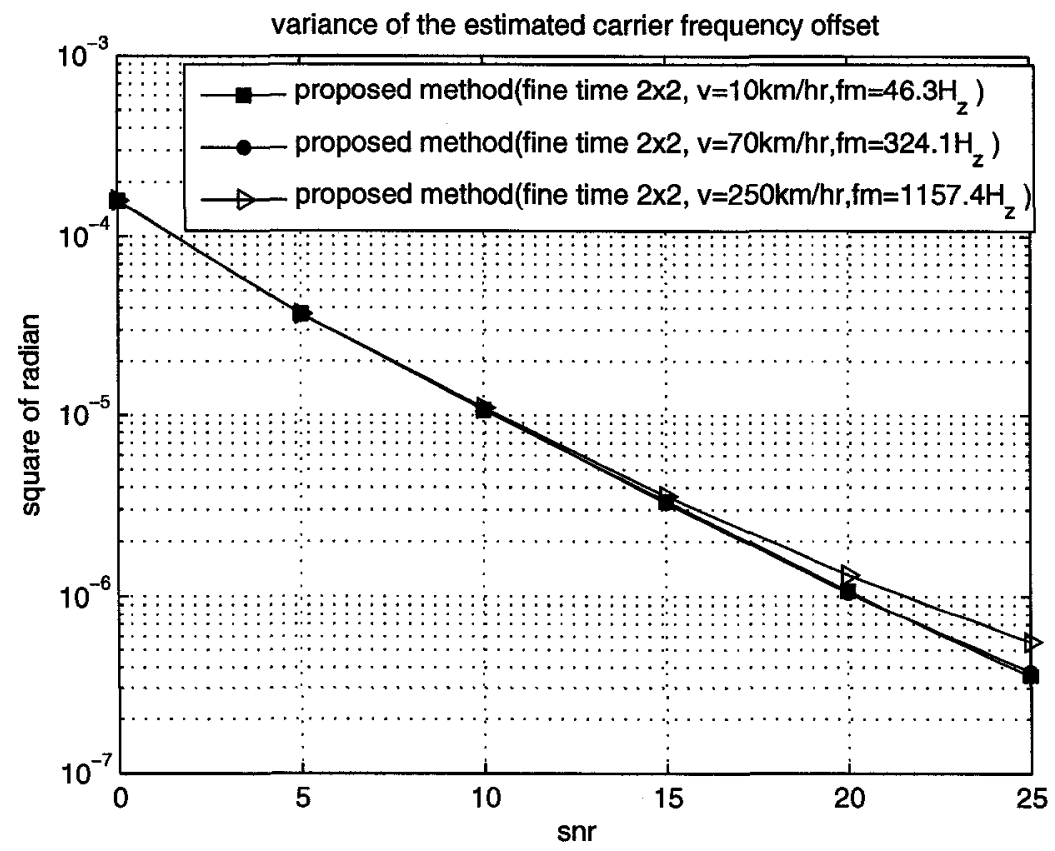

(b) Variance of the CFO

Fig. 5.36 Performance of the CFO estimator using EGC in the time-variant Rayleigh fading channel for various maximum Doppler frequencies, $Q=2, L=2$ 


\section{Chapter 6}

\section{Conclusions and future works}

\subsection{Conclusions}

This thesis presents a postfix synchronization method for OFDM and MIMO-OFDM systems that uses preambles containing two identical training parts (Chu sequences) followed by a Flipped Postfix (FIP). Specifically, the proposed preamble for AWGN channels consists of a Flipped Cyclic Prefix (FCP) and two identical training parts followed by a FlP. The FCP and FIP lengths are the Cyclic Prefix (CP) length of conventional preambles. The preamble for ISI or fading channels is similar except that the FCP is replaced by a Zero Padding Prefix (ZPP) of length $G / 2$ and each identical part

is a shorter Chu sequence of length $\frac{N}{2}-\frac{G}{2}$ followed by $\frac{G}{2}$ zeroes. For MIMO-OFDM systems, since the training sequences of different antennas should be orthogonal and shift-orthogonal and each training sequence of different antennas should have a perfect autocorrelation function in the fading channel, we use Chu sequences and each circular shift $G$ version of Chu sequences to be the half part of the first training symbol for each transmitter antenna. The first symbol consists of a half length CP and two identical training parts followed by a FlP. We analyze the effect of the FIP in AWGN 
channels. By using these FlP-based preambles, the accuracy of the timing estimator is highly improved compared to classical existing methods such as Schmidl and Cox's or Minn' methods without increasing complexity.

The proposed synchronization method is implemented in four steps. Step I is Coarse Time Synchronization. It uses the timing metric to find the coarse estimated symbol start point. Step II is Estimation of the Fraction Part of the CFO. It uses the phase difference between the two identical parts of the first training symbol. Since it uses the coarse estimated symbol start point as the symbol start point, its performance depends on the coarse time synchronization. Step III is Estimation of the Integer Part of the CFO. It uses the difference of the two training symbols on the even subcarriers. Specifically, after the fraction part of the CFO correction, the ratio sequence is retrieved and compared with the reference sequence in the frequency domain to get the estimated integer part of the CFO. Its performance depends on step I and step II. Step IV is Fine Time Synchronization. We calculate the crosscorrelation of the CFO corrected sample sequences and the transmitted preamble sequences in a short range whose center is the coarse estimated symbol start point. We search the first point in that short range whose cross-correlation is greater than the threshold (from smallest to greatest). Using the fine time synchronization algorithm, the accuracies of the proposed method and SC's method are much improved in the low SNR scenario by setting a proper threshold. We give a theoretical support for setting the threshold. Since the cross correlation cannot be calculated iteratively, the computation complexity is high. Since the searching range of the proposed method is shorter than the one of SC's method, the proposed fine time estimation method has less complexity than SC's fine time estimation method. In fading channels, the fine time estimator has an error floor when SNR increases. This error floor is caused by the probability of false alarm and missing detection of the fine timing estimator and 
depends on the threshold setting and tap fading. Since we can estimate SNR from the peak value of the timing metric, we can set up a look up table depending on the estimated SNR for setting the threshold. If we know the channel power delay profile, it is very helpful for setting the threshold. The performance is dominated by steps I, II and III in a low SNR scenario and by the probability of false alarm and missing detection of the fine timing estimator in a high SNR scenario.

MIMO-OFDM systems are also sensitive to synchronization errors. We extended the proposed method and SC's method to MIMO-OFDM systems. By using multiple antennas, the spatial diversity improves the accuracy of the symbol timing and carrier frequency offset estimator.

The performance of the proposed method for OFDM systems is evaluated by simulation over AWGN, ISI or fading channels in terms of the mean and variance of the symbol timing and carrier frequency offset estimators. Results show the proposed symbol timing estimator has a quite small variance and its mean value is quite close to the exact start point. Comparison to classical synchronization methods such as SC's and Minn's methods is also done. The performance of the proposed method for MIMO-OFDM systems is evaluated by simulation in fading channels. By comparing different combining techniques, such as EGC and MRC, we found the EGC had almost the same performance as MRC but is less complex. Looking from the point of view of synchronization, this thesis also shows that receiver diversity gives more gains than transmitter diversity.

In [29], the performance of the proposed method for OFDM systems is evaluated by simulation over AWGN, ISI and fading channels in terms of the mean and variance of the symbol timing and carrier frequency offset estimators using different simulation parameters. Obtained results are similar to the results presented in this thesis and can be seen as a complement of this thesis. 
From the analysis and simulations, it is seen that the proposed synchronization method has a narrow and large timing metric peak value suitable to specify a threshold and is robust in continuous or burst communication scenarios under different channels with large CFO. It is also robust to the Doppler spread. In particular, simulations in various channels have shown the proposed symbol timing estimator's mean value is quite close to the exact start point with a small variance from the left side of it. The accuracy of the proposed CFO estimator is similar in low SNR scenario and slightly worse in high SNR scenario than SC's CFO estimator one. Taking into consideration both timing synchronization and CFO correction, this thesis showed that postfix synchronization methods are suitable for the practical synchronization of OFDM or MIMO-OFDM systems in various channels.

\subsection{Future works}

The proposed method is a synchronization method in the acquisition mode. After finding the start of the frame and estimated CFO, we have to estimate the channel. To make the preamble efficient, we need an algorithm to estimate the channel by using the information contained in the training symbols.

From appendix A, the FlP-based preamble increases the mean value of $M_{d}$. If we boost the power of the FlP, the mean value of $M_{d}$ will be increased further. Future work could be to use power-boost FlP-based preambles to improve the accuracy of the symbol timing estimator under reasonable PAPR.

In this thesis, we assume a perfect sampling time. If there is a sampling clock offset, the performance of the proposed method will degrade. Using training symbols jointly to estimate the symbol timing, carrier frequency offset and sampling clock offset is another future work. 
Generalized multicarrier (GMC) [30] transmission systems also need reliable synchronization methods. Future work could consist of adapting the proposed FlP-based preambles synchronization method to GMC systems and evaluating its performance. 


\section{Appendix A}

\section{The distribution of}

$$
M_{d}=M_{p}\left(d_{o p t}\right)-M_{p}\left(d_{o p t+1}\right)
$$

Using FlP-based preambles can decrease the probability that the peak of metric shifts to the right side of the exact start point. Let us recall the timing metric formula:

$$
P_{p}(d)=\sum_{m=0}^{L_{p}-1}\left(r_{d+m}^{*} \cdot r_{d+m+L_{p}}\right)
$$

where $L_{p}=\frac{N}{2}$ samples and $N$ is the IFFT size. We use the energy of the second half of the first training symbol to normalize the sum of the correlation value. The energy is calculated as:

$$
R_{p}(d)=\sum_{m=0}^{L_{p}-1}\left|r_{d+m+L_{p}}\right|^{2}
$$

A timing metric is defined as the normalized sum of products and can be given as:

$$
M_{p}(d)=\frac{\left|P_{p}(d)\right|^{2}}{R_{p}^{2}(d)}
$$

Let us calculate the probability that the timing metric value of the exact start point 
is smaller than the one of the right shift one point. To calculate that probability, we have to calculate the distribution of the random variable $M_{d}=M_{p}\left(d_{\text {opt }}\right)-M_{p}\left(d_{\text {opt }+1}\right)$. From [6], we know the $\left|P_{p}(d)\right|, R_{p}(d)$, and $M_{p}(d)$ can be approximated by Gaussian Random Variables (RVs) from the central limit theorem (CLT). Since $\left|P_{p}(d)\right|$ and $R_{p}(d)$ have much larger means than variances, $\left|P_{p}(d)\right|^{2}$ and $R_{p}^{2}(d)$ can also be approximated by Gaussian RVs. Let each complex sample $r_{m}=s_{m}+n_{m}$ be made up of a signal and a noise component. Let the variance of the real and imaginary components be:

$$
\begin{gathered}
E\left[\Re\left\{s_{m}\right\}^{2}\right]=E\left[\Im\left\{s_{m}\right\}^{2}\right]=\frac{\sigma_{s}^{2}}{2} \\
E\left[\Re\left\{n_{m}\right\}^{2}\right]=E\left[\Im\left\{n_{m}\right\}^{2}\right]=\frac{\sigma_{n}^{2}}{2}
\end{gathered}
$$

SNR is $\frac{\sigma_{s}^{2}}{\sigma_{n}^{2}}$ and we have:

$$
\begin{aligned}
P_{p}(d) & =\sum_{m=0}^{L_{p}-1}\left(r_{d+m}^{*} \cdot r_{d+m+L_{p}}\right) \\
& =\sum_{m=0}^{L_{p}-1} s_{d+m}^{*} s_{d+m+L_{p}}+s_{d+m}^{*} n_{d+m+L_{p}}+s_{d+m+L_{p}} n_{d+m}^{*}+n_{d+m}^{*} n_{d+m+L_{p}}
\end{aligned}
$$

and

$$
\begin{aligned}
\left|P_{p}(d)\right| & \approx e^{-j \phi} \sum_{m=0}^{L_{p}-1} s_{d+m}^{*} s_{d+m+L_{p}} \\
& +\sum_{m=0}^{L_{p}-1} \operatorname{inPhase}_{\phi}\left(s_{d+m}^{*} n_{d+m+L_{p}}+s_{d+m+L_{p}} n_{d+m}^{*}+n_{d+m}^{*} n_{d+m+L_{p}}\right)
\end{aligned}
$$

where from (3.5) $\phi=\pi \gamma$. We have

$$
E\left\{s_{d_{o p t}+m}^{*} s_{d_{o p t}+m+L_{p}}\right\}=e^{j \phi} \sigma_{s}^{2}, \quad m=0,1, \cdots, L_{p}-1
$$




$$
\begin{aligned}
& E\left\{s_{d_{o p t}+m+L_{p}} s_{d_{o p t}+m+2 L_{p}}\right\} \\
& =\left\{\begin{aligned}
-e^{j \phi} \sigma_{s}^{2}, m=0,1, \cdots, G / 2 . & \text { using the FlP preamble. } \\
0, & m=0,1, \cdots, G / 2 .
\end{aligned}\right. \text { using SC's preamble. }
\end{aligned}
$$

$\left|P_{p}\left(d_{\text {opt }}\right)\right|$ is a Gaussian Random Variable $(\mathrm{RV})$ with mean $L_{p} \sigma_{s}^{2}$ and its variance is quite small compared to its mean. When we use FlP, $E\left\{s_{d_{o p t}+L_{p}}^{*} s_{d_{o p t}+2 L_{p}}\right\}=-e^{-j \phi} \sigma_{s}^{2}$, $\left|P_{p}\left(d_{o p t+1}\right)\right|$ is also a Gaussian RV but its mean is $\left(L_{p}-2\right) \sigma_{s}^{2}$. Its variance is also small compared to its mean when $L_{p}$ is large. In the conventional (SC) structure, $s_{d_{o p t}+2 L_{p}}$ would be the first CP sample of the next symbol, so $E\left\{s_{d_{o p t}+L_{p}}^{*} s_{d_{o p t}+2 L_{p}}\right\}=0$ and $\left|P_{p}\left(d_{o p t+1}\right)\right|$ 's mean is $\left(L_{p}-1\right) \sigma_{s}^{2}$.

For $R_{p}(d)$ we have

$$
\begin{aligned}
R_{p}(d) & =\sum_{m=0}^{L_{p}-1}\left|r_{d+m+L_{p}}\right|^{2} \\
& =\sum_{m=0}^{L_{p}-1}\left|s_{d+m+L_{p}}\right|^{2}+s_{d+m+L_{p}}^{*} n_{d+m+L_{p}}+s_{d+m+L_{p}} n_{d+m+L_{p}}^{*}+\left|n_{d+m+L_{p}}\right|^{2}
\end{aligned}
$$

$R_{p}(d)$ is also a Gaussian RV with mean $L_{p}\left(\sigma_{s}^{2}+\sigma_{n}^{2}\right)$ and its variance is quite small compared to its mean.

Since $\left|P_{p}(d)\right|$ and $R_{p}(d)$ have much larger means than their variances, $\left|P_{p}(d)\right|^{2}$ and $R_{p}^{2}(d)$ can also be approximated by Gaussian RVs with means $L_{p}^{2} \sigma_{s}^{4}, L_{p}^{2}\left(\sigma_{s}^{2}+\right.$ $\left.\sigma_{n}^{2}\right)^{2}$ respectively and their means are quite larger than their variances. Using the approximation $(1+b) /(1+c) \approx(1+b-c)$ (b and $c$ are zero mean Gaussian RVs and their variances are quite smaller than one) we have

$$
M_{p}\left(d_{o p t}\right)=\frac{\left|P_{p}\left(d_{o p t}\right)\right|^{2}}{R_{p}^{2}\left(d_{o p t}\right)} \approx \frac{L_{p}^{2} \sigma_{s}^{4}}{L_{p}^{2}\left(\sigma_{s}^{2}+\sigma_{n}^{2}\right)^{2}} \frac{1+\delta P_{p}}{1+\delta R_{p}}
$$




$$
\begin{aligned}
& \approx \frac{\sigma_{s}^{4}}{\left(\sigma_{s}^{2}+\sigma_{n}^{2}\right)^{2}}\left(1+\delta P_{p}-\delta R_{p}\right)=\frac{\sigma_{s}^{4}}{\left(\sigma_{s}^{2}+\sigma_{n}^{2}\right)^{2}}\left(1+\delta P_{p}-\delta R_{p}-1+1\right) \\
& =\frac{\left|P_{p}\left(d_{o p t}\right)\right|^{2}}{L_{p}^{2}\left(\sigma_{s}^{2}+\sigma_{n}^{2}\right)^{2}}-\frac{R_{p}^{2}\left(d_{o p t}\right) \sigma_{s}^{4}}{L_{p}^{2}\left(\sigma_{s}^{2}+\sigma_{n}^{2}\right)^{4}}+\frac{\sigma_{s}^{4}}{\left(\sigma_{s}^{2}+\sigma_{n}^{2}\right)^{2}}
\end{aligned}
$$

similarly we have

$$
M_{p}\left(d_{o p t+1}\right) \approx \frac{\left|P_{p}\left(d_{o p t+1}\right)\right|^{2}}{L_{p}^{2}\left(\sigma_{s}^{2}+\sigma_{n}^{2}\right)^{2}}-\frac{R_{p}^{2}\left(d_{o p t+1}\right) \sigma_{s}^{4}}{L_{p}^{2}\left(\sigma_{s}^{2}+\sigma_{n}^{2}\right)^{4}}+\frac{\sigma_{s}^{4}}{\left(\sigma_{s}^{2}+\sigma_{n}^{2}\right)^{2}}
$$

$\left(\left|P_{p}\left(d_{\text {opt }+1}\right)\right|^{2}\right.$ 's mean is $\left(L_{p}-2\right)^{2} \sigma_{s}^{4}$ or $\left(L_{p}-1\right)^{2} \sigma_{s}^{4}$. Since $L_{p}$ is large, we approximate $\left.L_{p}-2 \approx L_{p}, L_{p}-1 \approx L_{p}\right)$

$M_{p}\left(d_{\text {opt }}\right), M_{p}\left(d_{o p t+1}\right)$ are approximated by Gaussian RVs, so $M_{d}=M_{p}\left(d_{\text {opt }}\right)-$ $M_{p}\left(d_{o p t+1}\right)$ is also a Gaussian RV.

$$
\begin{aligned}
M_{d} & =M_{p}\left(d_{o p t}\right)-M_{p}\left(d_{o p t+1}\right) \\
& \approx \frac{\left|P_{p}\left(d_{o p t}\right)\right|^{2}-\left|P_{p}\left(d_{o p t+1}\right)\right|^{2}}{L_{p}^{2}\left(\sigma_{s}^{2}+\sigma_{n}^{2}\right)^{2}}+\frac{\sigma_{s}^{4}}{L_{p}^{2}\left(\sigma_{s}^{2}+\sigma_{n}^{2}\right)^{4}}\left(R_{p}^{2}\left(d_{o p t+1}\right)-R_{p}^{2}\left(d_{o p t}\right)\right)
\end{aligned}
$$

To calculate the mean and variance of the $M_{d}$, we have to calculate the means and variances of $P_{d}=\left|P_{p}\left(d_{o p t}\right)\right|^{2}-\left|P_{p}\left(d_{o p t+1}\right)\right|^{2}$ and $R_{d}=R_{p}^{2}\left(d_{o p t+1}\right)-R_{p}^{2}\left(d_{o p t}\right)$ and their covariance. Let $c_{1}=\frac{1}{L_{p}^{2}\left(\sigma_{s}^{2}+\sigma_{n}^{2}\right)^{2}}$ and $c_{2}=\frac{\sigma_{s}^{4}}{L_{p}^{2}\left(\sigma_{s}^{2}+\sigma_{n}^{2}\right)^{4}}$. We have

$$
\begin{gathered}
E\left\{M_{d}\right\}=c_{1} \cdot E\left\{P_{d}\right\}+c_{2} \cdot E\left\{R_{d}\right\} \\
\operatorname{Var}\left(M_{d}\right)=c_{1}^{2} \cdot \operatorname{Var}\left(P_{d}\right)+c_{2}^{2} \cdot \operatorname{Var}\left(R_{d}\right)+2 \cdot c_{1} \cdot c_{2} \cdot \operatorname{Cov}\left(P_{d}, R_{d}\right)
\end{gathered}
$$

First, let's calculate the mean and variance of $P_{d}$. Let us define

$$
d_{m}=s_{d_{o p t}+m}^{*} s_{d_{o p t}+m+L_{p}}+s_{d_{o p t}+m}^{*} n_{d_{o p t}+m+L_{p}}+s_{d_{o p t}+m+L_{p}} n_{d_{o p t}+m}^{*}+n_{d_{o p t}+m}^{*} n_{d_{o p t}+m+L_{p}}
$$


Then

$$
\begin{aligned}
\left|d_{m}\right|^{2}=d_{m}^{*} d_{m} & \\
= & \left(s_{d_{o p t}+m}^{*} s_{d_{o p t}+m+L_{p}}+s_{d_{o p t}+m}^{*} n_{d_{o p t}+m+L_{p}}+s_{d_{o p t}+m+L_{p}} n_{d_{o p t}+m}^{*}+n_{d_{o p t}+m}^{*} n_{d_{o p t}+m+L_{p}}\right)^{*} \\
\cdot & \left(s_{d_{o p t}+m}^{*} s_{d_{o p t}+m+L_{p}}+s_{d_{o p t}+m}^{*} n_{d_{o p t}+m+L_{p}}+s_{d_{o p t}+m+L_{p}} n_{d_{o p t}+m}^{*}+n_{d_{o p t}+m}^{*} n_{d_{o p t}+m+L_{p}}\right) \\
= & \left(s_{d_{o p t}+m} s_{d_{o p t}+m+L_{p}}^{*}+s_{d_{o p t}+m} n_{d_{o p t}+m+L_{p}}^{*}+s_{d_{o p t}+m+L_{p}}^{*} n_{d_{o p t}+m}+n_{d_{o p t}+m} n_{d_{o p t}+m+L_{p}}^{*}\right) \\
\cdot & \left(s_{d_{o p t}+m}^{*} s_{d_{o p t}+m+L_{p}}+s_{d_{o p t}+m}^{*} n_{d_{o p t}+m+L_{p}}+s_{d_{o p t}+m+L_{p}} n_{d_{o p t}+m}^{*}+n_{d_{o p t}+m}^{*} n_{d_{o p t}+m+L_{p}}\right) \\
= & \left|s_{d_{o p t}+m}\right|^{2}\left|s_{d_{o p t}+m+L_{p}}\right|^{2}+\left|s_{d_{o p t}+m}\right|^{2} n_{d_{o p t}+m+L_{p}}^{*} s_{d_{o p t}+m+L_{p}}+\left|s_{d_{o p t}+m+L_{p}}\right|^{2} s_{d_{o p t}+m}^{*} \\
& n_{d_{o p t}+m}+n_{d_{o p t}+m} n_{d_{o p t}+m+L_{p}}^{*} s_{d_{o p t}+m}^{*} s_{d_{o p t}+m+L_{p}}+\left|s_{d_{o p t}+m}\right|^{2} s_{d_{o p t}+m+L_{p}}^{*} n_{d_{o p t}+m+L_{p}} \\
& +\left|s_{d_{o p t}+m}\right|^{2}\left|n_{d_{o p t}+m+L_{p}}\right|^{2}+s_{d_{o p t}+m+L_{p}}^{*} n_{d_{o p t}+m} s_{d_{o p t}+m}^{*} n_{d_{o p t}+m+L_{p}} \\
& +n_{d_{o p t}+m} s_{d_{o p t}+m}^{*}\left|n_{d_{o p t}+m+L_{p}}\right|^{2}+s_{d_{o p t}+m}\left|s_{d_{o p t}+m+L_{p}}\right|^{2} n_{d_{o p t}+m}^{*} \\
& +s_{d_{o p t}+m} n_{d_{o p t}+m+L_{p}}^{*} s_{d_{o p t}+m+L_{p}} n_{d_{o p t}+m}^{*}+\left|s_{d_{o p t}+m+L_{p}}\right|^{2}\left|n_{d_{o p t}+m}\right|^{2} \\
& +\left|n_{d_{o p t}+m}\right|^{2} n_{d_{o p t}+m+L_{p}}^{*} s_{d_{o p t}+m+L_{p}}+s_{d_{o p t}+m} s_{d_{o p t}+m+L_{p}}^{*} n_{d_{o p t}+m}^{*} n_{d_{o p t}+m+L_{p}}+s_{d_{o p t}+m} \\
& n_{d_{o p t}+m}^{*}\left|n_{d_{o p t}+m+L_{p}}\right|^{2}+s_{d_{o p t}+m+L_{p}}^{*}\left|n_{d_{o p t}+m}\right|^{2} n_{d_{o p t}+m+L_{p}}+\left|n_{d_{o p t}+m}\right|^{2}\left|n_{d_{o p t}+m+L_{p}}\right|^{2}
\end{aligned}
$$

Using that the first training sequence is not random and that constant envelopes (Chu sequences) sequences are used for the training sequences, we have

$$
\begin{gathered}
E\left\{\left|d_{m}\right|^{2}\right\}=\sigma_{s}^{4}+2 \sigma_{s}^{2} \sigma_{n}^{2}+\sigma_{n}^{4} \\
P_{d}=\left|P_{p}\left(d_{o p t}\right)\right|^{2}-\left|P_{p}\left(d_{o p t+1}\right)\right|^{2} \\
=\left(d_{0}+\sum_{m=1}^{L_{p}-1} d_{m}\right)^{*}\left(d_{0}+\sum_{m=1}^{L_{p}-1} d_{m}\right)-\left(d_{L_{p}}+\sum_{m=1}^{L_{p}-1} d_{m}\right)^{*}\left(d_{L_{p}}+\sum_{m=1}^{L_{p}-1} d_{m}\right)
\end{gathered}
$$




$$
\begin{aligned}
= & \left|d_{0}\right|^{2}+d_{0}^{*} \sum_{m=1}^{L_{p}-1} d_{m}+d_{0} \sum_{m=1}^{L_{p}-1} d_{m}^{*}+\left|\sum_{m=1}^{L_{p}-1} d_{m}\right|^{2} \\
& -\left(\left|d_{L_{p}}\right|^{2}+d_{L_{p}}^{*} \sum_{m=1}^{L_{p}-1} d_{m}+d_{L_{p}} \sum_{m=1}^{L_{p}-1} d_{m}^{*}+\left|\sum_{m=1}^{L_{p}-1} d_{m}\right|^{2}\right) \\
= & \left|d_{0}\right|^{2}-\left|d_{L_{p}}\right|^{2}+\left(d_{0}^{*}-d_{L_{p}}^{*}\right) \sum_{m=1}^{L_{p}-1} d_{m}+\left(d_{0}-d_{L_{p}}\right) \sum_{m=1}^{L_{p}-1} d_{m}^{*} \\
= & \left|d_{0}\right|^{2}-\left|d_{L_{p}}\right|^{2}+2 \Re\left\{\left(d_{0}^{*}-d_{L_{p}}^{*}\right) \sum_{m=1}^{L_{p}-1} d_{m}\right\}
\end{aligned}
$$

From (A.8) and (A.9), we have

$$
E\left\{d_{m}^{*}\right\}=\left\{\begin{array}{lll}
e^{-j \phi} \sigma_{s}^{2}, & m=0,1, \cdots, L_{p}-1 . \\
-e^{-j \phi} \sigma_{s}^{2}, & m=L_{p} . & \text { using the FIP preamble. } \\
0, & m=L_{p} . & \text { using SC's preamble. }
\end{array}\right.
$$

Since $d_{0}, d_{L_{p}}$ are independent of $d_{m}, m=1,2, \cdots, L_{p}-1$ (noises at each sample are independent and $s_{d_{o p t}}, s_{d_{o p t}+L_{p}}, s_{d_{o p t}+2 L_{p}}$ are independent of $s_{d_{o p t}+m}, m=1,2, \cdots, 2 L_{p}$ 1. $m \neq L_{p}$ ), we have

$$
\begin{aligned}
& E\left\{\Re\left\{\left(d_{0}^{*}-d_{L_{p}}^{*}\right) \sum_{m=1}^{L_{p}-1} d_{m}\right\}\right\}=\Re\left\{E\left\{\left(d_{0}^{*}-d_{L_{p}}^{*}\right) \sum_{m=1}^{L_{p}-1} d_{m}\right\}\right\} \\
& =\Re\left\{E\left\{\left(d_{0}^{*}-d_{L_{p}}^{*}\right)\right\} \sum_{m=1}^{L_{p}-1} E\left\{d_{m}\right\}\right\}=\Re\left\{\left(E\left\{d_{0}^{*}\right\}-E\left\{d_{L_{p}}^{*}\right\}\right) \sum_{m=1}^{L_{p}-1} E\left\{d_{m}\right\}\right\} \\
& = \begin{cases}2\left(L_{p}-1\right) \sigma_{s}^{4} & \text { using the FlP preamble. } \\
\left(L_{p}-1\right) \sigma_{s}^{4} & \text { using SC's preamble. }\end{cases}
\end{aligned}
$$


From (A.18), (A.19) and (A.21), we have

$$
\begin{aligned}
E\left\{P_{d}\right\} & =E\left\{\left|d_{0}\right|^{2}\right\}-E\left\{\left|d_{L_{p}}\right|^{2}\right\}+2 E\left\{\Re\left\{\left(d_{0}^{*}-d_{L_{p}}^{*}\right) \sum_{m=1}^{L_{p}-1} d_{m}\right\}\right\} \\
& =\left\{\begin{array}{lc}
4\left(L_{p}-1\right) \sigma_{s}^{4} & \text { using the FlP preamble. } \\
2\left(L_{p}-1\right) \sigma_{s}^{4} & \text { using SC's preamble. }
\end{array}\right.
\end{aligned}
$$

To calculate $\operatorname{Var}\left(P_{d}\right)$, first we calculate the second moment of $P_{d}$. We have

$$
\begin{aligned}
\left|P_{d}\right|^{2}= & \left(\left|P_{p}\left(d_{o p t}\right)\right|^{2}-\left|P_{p}\left(d_{o p t+1}\right)\right|^{2}\right)^{*}\left(\left|P_{p}\left(d_{o p t}\right)\right|^{2}-\left|P_{p}\left(d_{o p t+1}\right)\right|^{2}\right) \\
= & {\left[\left|d_{0}\right|^{2}-\left|d_{L_{p}}\right|^{2}+\left(d_{0}^{*}-d_{L_{p}}^{*}\right) \sum_{m=1}^{L_{p}-1} d_{m}+\left(d_{0}-d_{L_{p}}\right) \sum_{m=1}^{L_{p}-1} d_{m}^{*}\right]^{*} } \\
& \cdot\left[\left|d_{0}\right|^{2}-\left|d_{L_{p}}\right|^{2}+\left(d_{0}^{*}-d_{L_{p}}^{*}\right) \sum_{m=1}^{L_{p}-1} d_{m}+\left(d_{0}-d_{L_{p}}\right) \sum_{m=1}^{L_{p}-1} d_{m}^{*}\right] \\
= & \left(\left|d_{0}\right|^{2}-\left|d_{L_{p}}\right|^{2}\right)^{2}+2\left(\left|d_{0}\right|^{2}-\left|d_{L_{p}}\right|^{2}\right) \cdot 2 \Re\left\{\left(d_{0}-d_{L_{p}}\right) \sum_{m=1}^{L_{p}-1} d_{m}^{*}\right\} \\
& +\left[\left(d_{0}^{*}-d_{L_{p}}^{*}\right) \sum_{m=1}^{L_{p}-1} d_{m}\right]^{2}+2\left|\left(d_{0}^{*}-d_{L_{p}}^{*}\right) \sum_{m=1}^{L_{p}-1} d_{m}\right|^{2}+\left[\left(d_{0}-d_{L_{p}}\right) \sum_{m=1}^{L_{p}-1} d_{m}^{*}\right]^{2}
\end{aligned}
$$

$$
\begin{aligned}
E\left\{\left|d_{m}\right|^{4}\right\} & =E\left\{\left|d_{m}\right|^{2} \cdot\left|d_{m}\right|^{2}\right\} \\
& =\left(\sigma_{s}^{4}+2 \sigma_{s}^{2} \sigma_{n}^{2}+\sigma_{n}^{4}\right)^{2}+\sigma_{s}^{6} \sigma_{n}^{2}+\sigma_{s}^{6} \sigma_{n}^{2}+\sigma_{s}^{4} \sigma_{n}^{4}+\sigma_{s}^{6} \sigma_{n}^{2}+\sigma_{s}^{4} \sigma_{n}^{4}+\sigma_{s}^{2} \sigma_{n}^{6} \\
& +\sigma_{s}^{6} \sigma_{n}^{2}+\sigma_{s}^{4} \sigma_{n}^{4}+\sigma_{s}^{2} \sigma_{n}^{6}+\sigma_{s}^{4} \sigma_{n}^{4}+\sigma_{s}^{2} \sigma_{n}^{6}+\sigma_{s}^{2} \sigma_{n}^{6}+2 \sigma_{s}^{6} \sigma_{n}^{2}+4 \sigma_{s}^{4} \sigma_{n}^{4}+2 \sigma_{s}^{2} \sigma_{n}^{6} \\
& =\left(\sigma_{s}^{4}+2 \sigma_{s}^{2} \sigma_{n}^{2}+\sigma_{n}^{4}\right)^{2}+6 \sigma_{s}^{6} \sigma_{n}^{2}+8 \sigma_{s}^{4} \sigma_{n}^{4}+6 \sigma_{s}^{2} \sigma_{n}^{6}
\end{aligned}
$$




$$
\begin{aligned}
& E\left\{\left|d_{0}\right|^{2}\left|d_{L_{p}}\right|^{2}\right\}=E\left\{\left(\left|s_{d_{o p t}}\right|^{2}\left|s_{d_{o p t}+L_{p}}\right|^{2}+\left|s_{d_{o p t}}\right|^{2} n_{d_{o p t}+L_{p}}^{*} s_{d_{o p t}+L_{p}}\right.\right. \\
& +\left|s_{d_{o p t}+L_{p}}\right|^{2} s_{d_{o p t}}^{*} n_{d_{o p t}}+n_{d_{o p t}} n_{d_{o p t}+L_{p}}^{*} s_{d_{o p t}}^{*} s_{d_{o p t}+L_{p}} \\
& +\left|s_{d_{o p t}}\right|^{2} s_{d_{o p t}+L_{p}}^{*} n_{d_{o p t}+L_{p}}+\left|s_{d_{o p t}}\right|^{2}\left|n_{d_{o p t}+L_{p}}\right|^{2} \\
& +s_{d_{o p t}+L_{p}}^{*} n_{d_{o p t}} s_{d_{o p t}}^{*} n_{d_{o p t}+L_{p}}+n_{d_{o p t}} s_{d_{o p t}}^{*}\left|n_{d_{o p t}+L_{p}}\right|^{2} \\
& +s_{d_{o p t}}\left|s_{d_{o p t}+L_{p}}\right|^{2} n_{d_{o p t}}^{*}+s_{d_{o p t}} n_{d_{o p t}+L_{p}}^{*} s_{d_{o p t}+L_{p}} n_{d_{o p t}}^{*} \\
& +\left|s_{d_{o p t}+L_{p}}\right|^{2}\left|n_{d_{o p t}}\right|^{2}+\left|n_{d_{o p t}}\right|^{2} n_{d_{o p t}+L_{p}}^{*} s_{d_{o p t}+L_{p}} \\
& +s_{d_{o p t}} s_{d_{o p t}+L_{p}}^{*} n_{d_{o p t}}^{*} n_{d_{o p t}+L_{p}}+s_{d_{o p t}} n_{d_{o p t}}^{*}\left|n_{d_{o p t}+L_{p}}\right|^{2} \\
& \left.+s_{d_{o p t}+L_{p}}^{*}\left|n_{d_{o p t}}\right|^{2} n_{d_{o p t}+L_{p}}+\left|n_{d_{o p t}}\right|^{2}\left|n_{d_{o p t}+L_{p}}\right|^{2}\right) \\
& \cdot\left(\left|s_{d_{o p t}+L_{p}}\right|^{2}\left|s_{d_{o p t}+2 L_{p}}\right|^{2}+\left|s_{d_{o p t}+L_{p}}\right|^{2} n_{d_{o p t}+2 L_{p}}^{*} s_{d_{o p t}+2 L_{p}}\right. \\
& +\left|s_{d_{o p t}+2 L_{p}}\right|^{2} s_{d_{o p t}+L_{p}}^{*} n_{d_{o p t}+L_{p}}+n_{d_{o p t}+L_{p}} n_{d_{o p t}+2 L_{p}}^{*} s_{d_{o p t}+L_{p}}^{*} s_{d_{o p t}+2 L_{p}} \\
& +\left|s_{d_{o p t}+L_{p}}\right|^{2} s_{d_{o p t}+2 L_{p}}^{*} n_{d_{o p t}+2 L_{p}}+\left|s_{d_{o p t}+L_{p}}\right|^{2}\left|n_{d_{o p t}+2 L_{p}}\right|^{2} \\
& +s_{d_{o p t}+2 L_{p}}^{*} n_{d_{o p t}+L_{p}} s_{d_{o p t}+L_{p}}^{*} n_{d_{o p t}+2 L_{p}}+n_{d_{o p t}+L_{p}} s_{d_{o p t}+L_{p}}^{*}\left|n_{d_{o p t}+2 L_{p}}\right|^{2} \\
& +s_{d_{o p t}+L_{p}}\left|s_{d_{o p t}+2 L_{p}}\right|^{2} n_{d_{o p t}+L_{p}}^{*}+s_{d_{o p t}+L_{p}} n_{d_{o p t}+2 L_{p}}^{*} s_{d_{o p t}+2 L_{p}} n_{d_{o p t}+L_{p}}^{*} \\
& +\left|s_{d_{o p t}+2 L_{p}}\right|^{2}\left|n_{d_{o p t}+L_{p}}\right|^{2}+\left|n_{d_{o p t}+L_{p}}\right|^{2} n_{d_{o p t}+2 L_{p}}^{*} s_{d_{o p t}+2 L_{p}} \\
& +s_{d_{o p t}+L_{p}} s_{d_{o p t}+2 L_{p}}^{*} n_{d_{o p t}+L_{p}}^{*} n_{d_{o p t}+2 L_{p}}+s_{d_{o p t}+L_{p}} n_{d_{o p t}+L_{p}}^{*}\left|n_{d_{o p t}+2 L_{p}}\right|^{2} \\
& \left.\left.+s_{d_{o p t}+2 L_{p}}^{*}\left|n_{d_{o p t}+L_{p}}\right|^{2} n_{d_{o p t}+2 L_{p}}+\left|n_{d_{o p t}+L_{p}}\right|^{2}\left|n_{d_{o p t}+2 L_{p}}\right|^{2}\right)\right\} \\
& =\left(\sigma_{s}^{4}+2 \sigma_{s}^{2} \sigma_{n}^{2}+\sigma_{n}^{4}\right)^{2}+2 \sigma_{s}^{6} \sigma_{n}^{2}+4 \sigma_{s}^{4} \sigma_{n}^{4}+2 \sigma_{s}^{2} \sigma_{n}^{6} \\
& E\left\{\left(\left|d_{0}\right|^{2}-\left|d_{L_{p}}\right|^{2}\right)^{2}\right\}=E\left\{\left|d_{0}\right|^{4}\right\}-2 E\left\{\left|d_{0}\right|^{2}\left|d_{L_{p}}\right|^{2}\right\}+E\left\{\left|d_{L_{p}}\right|^{4}\right\} \\
& =2\left[\left(\sigma_{s}^{4}+2 \sigma_{s}^{2} \sigma_{n}^{2}+\sigma_{n}^{4}\right)^{2}+6 \sigma_{s}^{6} \sigma_{n}^{2}+8 \sigma_{s}^{4} \sigma_{n}^{4}+6 \sigma_{s}^{2} \sigma_{n}^{6}\right] \\
& -2\left[\left(\sigma_{s}^{4}+2 \sigma_{s}^{2} \sigma_{n}^{2}+\sigma_{n}^{4}\right)^{2}+2 \sigma_{s}^{6} \sigma_{n}^{2}+4 \sigma_{s}^{4} \sigma_{n}^{4}+2 \sigma_{s}^{2} \sigma_{n}^{6}\right] \\
& =8 \sigma_{s}^{6} \sigma_{n}^{2}+8 \sigma_{s}^{4} \sigma_{n}^{4}+8 \sigma_{s}^{2} \sigma_{n}^{6}
\end{aligned}
$$




$$
\begin{aligned}
& E\left\{\left|d_{m}\right|^{2} d_{m}\right\}=E\left\{\left(\left|s_{d_{o p t}+m}\right|^{2}\left|s_{d_{o p t}+m+L_{p}}\right|^{2}+\left|s_{d_{o p t}+m}\right|^{2} n_{d_{o p t}+m+L_{p}}^{*} s_{d_{o p t}+m+L_{p}}\right.\right. \\
& +\left|s_{d_{o p t}+m+L_{p}}\right|^{2} s_{d_{o p t}+m}^{*} n_{d_{o p t}+m}+n_{d_{o p t}+m} n_{d_{o p t}+m+L_{p}}^{*} s_{d_{o p t}+m}^{*} s_{d_{o p t}+m+L_{p}} \\
& +\left|s_{d_{o p t}+m}\right|^{2} s_{d_{o p t}+m+L_{p}}^{*} n_{d_{o p t}+m+L_{p}}+\left|s_{d_{o p t}+m}\right|^{2}\left|n_{d_{o p t}+m+L_{p}}\right|^{2} \\
& +s_{d_{o p t}+m+L_{p}}^{*} n_{d_{o p t}+m} s_{d_{o p t}+m}^{*} n_{d_{o p t}+m+L_{p}}+n_{d_{o p t}+m} s_{d_{o p t}+m}^{*}\left|n_{d_{o p t}+m+L_{p}}\right|^{2} \\
& +s_{d_{o p t}+m}\left|s_{d_{o p t}+m+L_{p}}\right|^{2} n_{d_{o p t}+m}^{*}+s_{d_{o p t}+m} n_{d_{o p t}+m+L_{p}}^{*} s_{d_{o p t}+m+L_{p}} n_{d_{o p t}+m}^{*} \\
& +\left|s_{d_{o p t}+m+L_{p}}\right|^{2}\left|n_{d_{o p t}+m}\right|^{2}+\left|n_{d_{o p t}+m}\right|^{2} n_{d_{o p t}+m+L_{p}}^{*} s_{d_{o p t}+m+L_{p}} \\
& +s_{d_{o p t}+m} s_{d_{o p t}+m+L_{p}}^{*} n_{d_{o p t}+m}^{*} n_{d_{o p t}+m+L_{p}}+s_{d_{o p t}+m} n_{d_{o p t}+m}^{*}\left|n_{d_{o p t}+m+L_{p}}\right|^{2} \\
& \left.+s_{d_{o p t}+m+L_{p}}^{*}\left|n_{d_{o p t}+m}\right|^{2} n_{d_{o p t}+m+L_{p}}+\left|n_{d_{o p t}+m}\right|^{2}\left|n_{d_{o p t}+m+L_{p}}\right|^{2}\right) \text {. } \\
& \left.\left(s_{d_{o p t}+m}^{*} s_{d_{o p t}+m+L_{p}}+s_{d_{o p t}+m}^{*} n_{d_{o p t}+m+L_{p}}+s_{d_{o p t}+m+L_{p}} n_{d_{o p t}+m}^{*}+n_{d_{o p t}+m}^{*} n_{d_{o p t}+m+L_{p}}\right)\right\} \\
& =\left(\sigma_{s}^{4}+2 \sigma_{s}^{2} \sigma_{n}^{2}+\sigma_{n}^{4}\right) E\left\{s_{d_{o p t}+m}^{*} s_{d_{o p t}+m+L_{p}}\right\}+\left(\sigma_{s}^{2} \sigma_{n}^{2}+\sigma_{n}^{4}\right) E\left\{s_{d_{o p t}+m}^{*} s_{d_{o p t}+m+L_{p}}\right\} \\
& +\left(\sigma_{s}^{2} \sigma_{n}^{2}+\sigma_{n}^{4}\right) E\left\{s_{d_{o p t}+m}^{*} s_{d_{o p t}+m+L_{p}}\right\}+\sigma_{n}^{4} E\left\{s_{d_{o p t}+m}^{*} s_{d_{o p t}+m+L_{p}}\right\} \\
& =\left(\sigma_{s}^{4}+4 \sigma_{s}^{2} \sigma_{n}^{2}+4 \sigma_{n}^{4}\right) E\left\{s_{d_{o p t}+m}^{*} s_{d_{o p t}+m+L_{p}}\right\} \\
& = \begin{cases}e^{j \phi} \sigma_{s}^{2}\left(\sigma_{s}^{4}+4 \sigma_{s}^{2} \sigma_{n}^{2}+4 \sigma_{n}^{4}\right), & m=0,1, \cdots, L_{p}-1 . \\
-e^{j \phi} \sigma_{s}^{2}\left(\sigma_{s}^{4}+4 \sigma_{s}^{2} \sigma_{n}^{2}+4 \sigma_{n}^{4}\right), & m=L_{p} . \quad \text { using the FlP preamble. } \\
0, & m=L_{p} . \quad \text { using SC's preamble. }\end{cases}
\end{aligned}
$$




$$
\begin{aligned}
& E\left\{\left|d_{0}\right|^{2} d_{L_{p}}\right\}=E\left\{\left(\left|s_{d_{o p t}}\right|^{2}\left|s_{d_{o p t}+L_{p}}\right|^{2}+\left|s_{d_{o p t}}\right|^{2} n_{d_{o p t}+L_{p}}^{*} s_{d_{o p t}+L_{p}}+\left|s_{d_{o p t}+L_{p}}\right|^{2} s_{d_{o p t}}^{*} n_{d_{o p t}}\right.\right. \\
& +n_{d_{o p t}} n_{d_{o p t}+L_{p}}^{*} s_{d_{o p t}}^{*} s_{d_{o p t}+L_{p}}+\left|s_{d_{o p t}}\right|^{2} s_{d_{o p t}+L_{p}}^{*} n_{d_{o p t}+L_{p}}+\left|s_{d_{o p t}}\right|^{2}\left|n_{d_{o p t}+L_{p}}\right|^{2} \\
& +s_{d_{o p t}+L_{p}}^{*} n_{d_{o p t}} s_{d_{o p t}}^{*} n_{d_{o p t}+L_{p}}+n_{d_{o p t}} s_{d_{o p t}}^{*}\left|n_{d_{o p t}+L_{p}}\right|^{2}+s_{d_{o p t}}\left|s_{d_{o p t}+L_{p}}\right|^{2} n_{d_{o p t}}^{*} \\
& +s_{d_{o p t}} n_{d_{o p t}+L_{p}}^{*} s_{d_{o p t}+L_{p}} n_{d_{o p t}}^{*}+\left|s_{d_{o p t}+L_{p}}\right|^{2}\left|n_{d_{o p t}}\right|^{2}+\left|n_{d_{o p t}}\right|^{2} n_{d_{o p t}+L_{p}}^{*} s_{d_{o p t}+L_{p}} \\
& +s_{d_{o p t}} s_{d_{o p t}+L_{p}}^{*} n_{d_{o p t}}^{*} n_{d_{o p t}+L_{p}}+s_{d_{o p t}} n_{d_{o p t}}^{*}\left|n_{d_{o p t}+L_{p}}\right|^{2} \\
& \left.+s_{d_{o p t}+L_{p}}^{*}\left|n_{d_{o p t}}\right|^{2} n_{d_{o p t}+L_{p}}+\left|n_{d_{o p t}}\right|^{2}\left|n_{d_{o p t}+L_{p}}\right|^{2}\right) \\
& \left.\cdot\left(s_{d_{o p t}+L_{p}}^{*} s_{d_{o p t}+2 L_{p}}+s_{d_{o p t}+L_{p}}^{*} n_{d_{o p t}+2 L_{p}}+s_{d_{o p t}+2 L_{p}} n_{d_{o p t}+L_{p}}^{*}+n_{d_{o p t}+L_{p}}^{*} n_{d_{o p t}+2 L_{p}}\right)\right\} \\
& =\left(\sigma_{s}^{4}+2 \sigma_{s}^{2} \sigma_{n}^{2}+\sigma_{n}^{4}\right) E\left\{s_{d_{o p t}+L_{p}}^{*} s_{d_{o p t}+2 L_{p}}\right\}+\left(\sigma_{s}^{2} \sigma_{n}^{2}+\sigma_{n}^{4}\right) E\left\{s_{d_{o p t}+L_{p}}^{*} s_{d_{o p t}+2 L_{p}}\right\} \\
& =\left(\sigma_{s}^{4}+3 \sigma_{s}^{2} \sigma_{n}^{2}+2 \sigma_{n}^{4}\right) E\left\{s_{d_{o p t}+L_{p}}^{*} s_{d_{o p t}+2 L_{p}}\right\} \\
& = \begin{cases}-e^{j \phi} \sigma_{s}^{2}\left(\sigma_{s}^{4}+3 \sigma_{s}^{2} \sigma_{n}^{2}+2 \sigma_{n}^{4}\right), & \text { using the FIP preamble. } \\
0, & \text { using SC's preamble. }\end{cases} \\
& E\left\{\left|d_{L_{p}}\right|^{2} d_{0}\right\}=E\left\{\left(\left|s_{d_{o p t}+L_{p}}\right|^{2}\left|s_{d_{o p t}+2 L_{p}}\right|^{2}+\left|s_{d_{o p t}+L_{p}}\right|^{2} n_{d_{o p t}+2 L_{p}}^{*} s_{d_{o p t}+2 L_{p}}\right.\right. \\
& +\left|s_{d_{o p t}+2 L_{p}}\right|^{2} s_{d_{o p t}+L_{p}}^{*} n_{d_{o p t}+L_{p}}+n_{d_{o p t}+L_{p}} n_{d_{o p t}+2 L_{p}}^{*} s_{d_{o p t}+L_{p}}^{*} s_{d_{o p t}+2 L_{p}} \\
& +\left|s_{d_{o p t}+L_{p}}\right|^{2} s_{d_{o p t}+2 L_{p}}^{*} n_{d_{o p t}+2 L_{p}}+\left|s_{d_{o p t}+L_{p}}\right|^{2}\left|n_{d_{o p t}+2 L_{p}}\right|^{2} \\
& +s_{d_{o p t}+2 L_{p}}^{*} n_{d_{o p t}+L_{p}} s_{d_{o p t}+L_{p}}^{*} n_{d_{o p t}+2 L_{p}}+n_{d_{o p t}+L_{p}} s_{d_{o p t}+L_{p}}^{*}\left|n_{d_{o p t}+2 L_{p}}\right|^{2} \\
& +s_{d_{o p t}+L_{p}}\left|s_{d_{o p t}+2 L_{p}}\right|^{2} n_{d_{o p t}+L_{p}}^{*}+s_{d_{o p t}+L_{p}} n_{d_{o p t}+2 L_{p}}^{*} s_{d_{o p t}+2 L_{p}} n_{d_{o p t}+L_{p}}^{*} \\
& +\left|s_{d_{o p t}+2 L_{p}}\right|^{2}\left|n_{d_{o p t}+L_{p}}\right|^{2}+\left|n_{d_{o p t}+L_{p}}\right|^{2} n_{d_{o p t}+2 L_{p}}^{*} s_{d_{o p t}+2 L_{p}} \\
& +s_{d_{o p t}+L_{p}} s_{d_{o p t}+2 L_{p}}^{*} n_{d_{o p t}+L_{p}}^{*} n_{d_{o p t}+2 L_{p}}+s_{d_{o p t}+L_{p}} n_{d_{o p t}+L_{p}}^{*}\left|n_{d_{o p t}+2 L_{p}}\right|^{2} \\
& \left.+s_{d_{o p t}+2 L_{p}}^{*}\left|n_{d_{o p t}+L_{p}}\right|^{2} n_{d_{o p t}+2 L_{p}}+\left|n_{d_{o p t}+L_{p}}\right|^{2}\left|n_{d_{o p t}+2 L_{p}}\right|^{2}\right) \\
& \left.\cdot\left(s_{d_{o p t}}^{*} s_{d_{o p t}+L_{p}}+s_{d_{o p t}}^{*} n_{d_{o p t}+L_{p}}+s_{d_{o p t}+L_{p}} n_{d_{o p t}}^{*}+n_{d_{o p t}}^{*} n_{d_{o p t}+L_{p}}\right)\right\} \\
& =\left(\sigma_{s}^{4}+2 \sigma_{s}^{2} \sigma_{n}^{2}+\sigma_{n}^{4}\right) E\left\{s_{d_{o p t}}^{*} s_{d_{o p t}+L_{p}}\right\}+\left(\sigma_{s}^{2} \sigma_{n}^{2}+\sigma_{n}^{4}\right) E\left\{s_{d_{o p t}}^{*} s_{d_{o p t}+L_{p}}\right\} \\
& =\left(\sigma_{s}^{4}+3 \sigma_{s}^{2} \sigma_{n}^{2}+2 \sigma_{n}^{4}\right) E\left\{s_{d_{o p t}}^{*} s_{d_{o p t}+L_{p}}\right\}=e^{j \phi} \sigma_{s}^{2}\left(\sigma_{s}^{4}+3 \sigma_{s}^{2} \sigma_{n}^{2}+2 \sigma_{n}^{4}\right)
\end{aligned}
$$




$$
E\left\{\sum_{m=1}^{L_{p}-1} d_{m}^{*}\right\}=\sum_{m=1}^{L_{p}-1} E\left\{d_{m}^{*}\right\}=e^{-j \phi} \sigma_{s}^{2}\left(L_{p}-1\right)
$$

Since $d_{0}, d_{L_{p}}$ are independent of $d_{m}, m=1,2, \cdots, L_{p}-1$, we have

$$
\begin{aligned}
& E\left\{2\left(\left|d_{0}\right|^{2}-\left|d_{L_{p}}\right|^{2}\right) \cdot 2 \Re\left\{\left(d_{0}-d_{L_{p}}\right) \sum_{m=1}^{L_{p}-1} d_{m}^{*}\right\}\right\} \\
& =4 \Re\left\{E\left\{\left(\left|d_{0}\right|^{2}-\left|d_{L_{p}}\right|^{2}\right)\left(d_{0}-d_{L_{p}}\right) \sum_{m=1}^{L_{p}-1} d_{m}^{*}\right\}\right\} \\
& =4 \Re\left\{E\left\{\left(\left|d_{0}\right|^{2}-\left|d_{L_{p}}\right|^{2}\right)\left(d_{0}-d_{L_{p}}\right)\right\} E\left\{\sum_{m=1}^{L_{p}-1} d_{m}^{*}\right\}\right\} \\
& =4 \Re\left\{\left(E\left\{\left|d_{0}\right|^{2} d_{0}\right\}+E\left\{\left|d_{L_{p}}\right|^{2} d_{L_{p}}\right\}-E\left\{\left|d_{0}\right|^{2} d_{L_{p}}\right\}-E\left\{\left|d_{L_{p}}\right|^{2} d_{0}\right\}\right)\left(e^{-j \phi} \sigma_{s}^{2}\left(L_{p}-1\right)\right)\right\} \\
& = \begin{cases}0, & \text { using the FlP preamble. } \\
4\left(L_{p}-1\right) \sigma_{s}^{4}\left(\sigma_{s}^{2} \sigma_{n}^{2}+2 \sigma_{n}^{4}\right), & \text { using SC's preamble. }\end{cases}
\end{aligned}
$$

Since $d_{0}, d_{L_{p}}$ are independent of $d_{m}, m=1,2, \cdots, L_{p}-1$, we have

$$
\begin{aligned}
& E\left\{\left(d_{0}-d_{L_{p}}\right)^{2}\left(\sum_{m=1}^{L_{p}-1} d_{m}^{*}\right)^{2}\right\}=E\left\{\left(d_{0}-d_{L_{p}}\right)^{2}\right\} E\left\{\left(\sum_{m=1}^{L_{p}-1} d_{m}^{*}\right)^{2}\right\} . \\
& E\left\{\left(d_{0}-d_{L_{p}}\right)^{2}\right\}=E\left\{\left(s_{d_{o p t}}^{*} s_{d_{o p t}+L_{p}}+s_{d_{o p t}}^{*} n_{d_{o p t}+L_{p}}+s_{d_{o p t}+L_{p}} n_{d_{o p t}}^{*}+n_{d_{o p t}}^{*} n_{d_{o p t}+L_{p}}\right.\right. \\
& \left.\left.-s_{d_{o p t}+L_{p}}^{*} s_{d_{o p t}+2 L_{p}}-s_{d_{o p t}+L_{p}}^{*} n_{d_{o p t}+2 L_{p}}-s_{d_{o p t}+2 L_{p}} n_{d_{o p t}+L_{p}}^{*}-n_{d_{o p t}+L_{p}}^{*} n_{d_{o p t}+2 L_{p}}\right)^{2}\right\} \\
& =E\left\{\left(s_{d_{o p t}}^{*} s_{d_{o p t}+L_{p}}\right)^{2}\right\}-2 E\left\{\left(s_{d_{o p t}}^{*} s_{d_{o p t}+L_{p}} s_{d_{o p t}+L_{p}}^{*} s_{d_{o p t}+2 L_{p}}\right\}\right. \\
& +E\left\{\left(s_{d_{o p t}+L_{p}}^{*} s_{d_{o p t}+2 L_{p}}\right)^{2}\right\}-2 E\left\{s_{d_{o p t}}^{*} n_{d_{o p t}+L_{p}} s_{d_{o p t}+2 L_{p}} n_{d_{o p t}+L_{p}}^{*}\right\} \\
& =e^{j 2 \phi} \sigma_{s}^{4}-2\left(\sigma_{s}^{2}+\sigma_{n}^{2}\right) E\left\{s_{d_{o p t}}^{*} s_{d_{o p t}+2 L_{p}}\right\}+E\left\{\left(s_{d_{o p t}+L_{p}}^{*} s_{d_{o p t}+2 L_{p}}\right)^{2}\right\} \\
& =\left\{\begin{array}{cc}
2 e^{j 2 \phi}\left(2 \sigma_{s}^{4}+\sigma_{s}^{2} \sigma_{n}^{2}\right), & \text { using the FlP preamble. } \\
e^{j 2 \phi} \sigma_{s}^{4}, & \text { using SC's preamble. }
\end{array}\right.
\end{aligned}
$$

As discussed above, $\sum_{m=1}^{L_{p}-1} d_{m}^{*}$ can be approximated by a Gaussian RV 
$\mathcal{C N}\left(e^{-j \phi} \sigma_{s}^{2}\left(L_{p}-1\right), \sigma_{c}^{2}\right)$. Its variance is quite smaller than its mean. We have

$$
\begin{aligned}
E\left\{\left(\sum_{m=1}^{L_{p}-1} d_{m}^{*}\right)^{2}\right\} & \approx E\left\{\left[e^{-j \phi} \sigma_{s}^{2}\left(L_{p}-1\right)\right]^{2}+2 e^{-j \phi} \sigma_{s}^{2}\left(L_{p}-1\right) \mathcal{C N}\left(0, \sigma_{c}^{2}\right)+\mathcal{C N}^{2}\left(0, \sigma_{c}^{2}\right)\right\} \\
& \approx e^{-j 2 \phi} \sigma_{s}^{4}\left(L_{p}-1\right)^{2}
\end{aligned}
$$

$$
\begin{aligned}
& E\left\{\left(d_{0}-d_{L_{p}}\right)^{2}\left(\sum_{m=1}^{L_{p}-1} d_{m}^{*}\right)^{2}\right\}=E\left\{\left(d_{0}-d_{L_{p}}\right)^{2}\right\} E\left\{\left(\sum_{m=1}^{L_{p}-1} d_{m}^{*}\right)^{2}\right\} \\
& =\left\{\begin{array}{lr}
2\left(2 \sigma_{s}^{4}+\sigma_{s}^{2} \sigma_{n}^{2}\right) \sigma_{s}^{4}\left(L_{p}-1\right)^{2}, & \text { using the FlP preamble. } \\
\sigma_{s}^{8}\left(L_{p}-1\right)^{2}, & \text { using SC's preamble. }
\end{array}\right.
\end{aligned}
$$

$$
\begin{aligned}
& E\left\{\left(d_{0}^{*}-d_{L_{p}}^{*}\right)^{2}\left(\sum_{m=1}^{L_{p}-1} d_{m}\right)^{2}\right\}=E\left\{\left(d_{0}-d_{L_{p}}\right)^{2}\left(\sum_{m=1}^{L_{p}-1} d_{m}^{*}\right)^{2}\right\}^{*} \\
& =\left\{\begin{array}{lr}
2\left(2 \sigma_{s}^{4}+\sigma_{s}^{2} \sigma_{n}^{2}\right) \sigma_{s}^{4}\left(L_{p}-1\right)^{2}, & \text { using the FIP preamble. } \\
\sigma_{s}^{8}\left(L_{p}-1\right)^{2}, & \text { using SC's preamble. }
\end{array}\right.
\end{aligned}
$$

Now let us calculate $E\left\{\left|\left(d_{0}^{*}-d_{L_{p}}^{*}\right) \sum_{m=1}^{L_{p}-1} d_{m}\right|^{2}\right\}$. We have

$$
\begin{aligned}
\left|\left(d_{0}^{*}-d_{L_{p}}^{*}\right) \sum_{m=1}^{L_{p}-1} d_{m}\right|^{2} & =\left[\left(d_{0}^{*}-d_{L_{p}}^{*}\right) \sum_{m=1}^{L_{p}-1} d_{m}\right]^{*}\left[\left(d_{0}^{*}-d_{L_{p}}^{*}\right) \sum_{n=1}^{L_{p}-1} d_{n}\right] \\
& =\left(\left|d_{0}\right|^{2}-d_{0} d_{L_{p}}^{*}-d_{0}^{*} d_{L_{p}}+\left|d_{L_{p}}\right|^{2}\right)\left(\sum_{m=1}^{L_{p}-1} d_{m}^{*} \sum_{n=1}^{L_{p}-1} d_{n}\right)
\end{aligned}
$$




$$
\begin{aligned}
E\left\{d_{0} d_{L_{p}}^{*}\right\} & =E\left\{\left(s_{d_{o p t}}^{*} s_{d_{o p t}+L_{p}}+s_{d_{o p t}}^{*} n_{d_{o p t}+L_{p}}+s_{d_{o p t}+L_{p}} n_{d_{o p t}}^{*}+n_{d_{o p t}}^{*} n_{d_{o p t}+L_{p}}\right)\right. \\
& \left.\left(s_{d_{o p t}+L_{p}} s_{d_{o p t}+2 L_{p}}^{*}+s_{d_{o p t}+L_{p}} n_{d_{o p t}+2 L_{p}}^{*}+s_{d_{o p t}+2 L_{p}}^{*} n_{d_{o p t}+L_{p}}+n_{d_{o p t}+L_{p}} n_{d_{o p t}+2 L_{p}}^{*}\right)\right\} \\
& = \begin{cases}-\sigma_{s}^{4}, & \text { using the FIP preamble. } \\
0, & \text { using SC's preamble. }\end{cases}
\end{aligned}
$$

$$
E\left\{d_{0}^{*} d_{L_{p}}\right\}=\left(E\left\{d_{0} d_{L_{p}}^{*}\right\}\right)^{*}= \begin{cases}-\sigma_{s}^{4}, & \text { using the FIP preamble } \\ 0, & \text { using SC's preamble }\end{cases}
$$

If $n \neq m, n=1,2, \cdots, L_{p}-1$ and $m=1,2, \cdots, L_{p}-1$, we assume that $d_{m}$ and $d_{n}$ are independent.

$$
\begin{aligned}
E\left\{\sum_{m=1}^{L_{p}-1} d_{m}^{*} \sum_{n=1}^{L_{p}-1} d_{n}\right\} & =E\left\{\sum_{m=1}^{L_{p}-1}\left|d_{m}\right|^{2}\right\}+E\left\{\sum_{m=1}^{L_{p}-1} d_{m}^{*} \sum_{n=1, n \neq m}^{L_{p}-1} d_{n}\right\} \\
& =\sum_{m=1}^{L_{p}-1} E\left\{\left|d_{m}\right|^{2}\right\}+\sum_{m=1}^{L_{p}-1} E\left\{d_{m}^{*}\right\} \sum_{n=1, n \neq m}^{L_{p}-1} E\left\{d_{n}\right\} \\
& =\left(L_{p}-1\right)\left(\sigma_{s}^{4}+2 \sigma_{s}^{2} \sigma_{n}^{2}+\sigma_{n}^{4}\right)+\left(L_{p}-1\right)\left(L_{p}-2\right) \sigma_{s}^{4}
\end{aligned}
$$

Since $d_{0}, d_{L_{p}}$ are independent of $d_{m}, m=1,2, \cdots, L_{p}-1$, we have

$$
\begin{aligned}
& E\left\{\left|\left(d_{0}^{*}-d_{L_{p}}^{*}\right) \sum_{m=1}^{L_{p}-1} d_{m}\right|^{2}\right\}=E\left\{\left(\left|d_{0}\right|^{2}-d_{0} d_{L_{p}}^{*}-d_{0}^{*} d_{L_{p}}+\left|d_{L_{p}}\right|^{2}\right)\left(\sum_{m=1}^{L_{p}-1} d_{m}^{*} \sum_{n=1}^{L_{p}-1} d_{n}\right)\right\} \\
& =E\left\{\left|d_{0}\right|^{2}-d_{0} d_{L_{p}}^{*}-d_{0}^{*} d_{L_{p}}+\left|d_{L_{p}}\right|^{2}\right\} E\left\{\sum_{m=1}^{L_{p}-1} d_{m}^{*} \sum_{n=1}^{L_{p}-1} d_{n}\right\}
\end{aligned}
$$




$$
=\left\{\begin{array}{c}
2\left(2 \sigma_{s}^{4}+2 \sigma_{s}^{2} \sigma_{n}^{2}+\sigma_{n}^{4}\right)\left[\left(L_{p}-1\right)\left(\sigma_{s}^{4}+2 \sigma_{s}^{2} \sigma_{n}^{2}+\sigma_{n}^{4}\right)+\left(L_{p}-1\right)\left(L_{p}-2\right) \sigma_{s}^{4}\right] \\
\text { using the FIP preamble. } \\
2\left(\sigma_{s}^{4}+2 \sigma_{s}^{2} \sigma_{n}^{2}+\sigma_{n}^{4}\right)\left[\left(L_{p}-1\right)\left(\sigma_{s}^{4}+2 \sigma_{s}^{2} \sigma_{n}^{2}+\sigma_{n}^{4}\right)+\left(L_{p}-1\right)\left(L_{p}-2\right) \sigma_{s}^{4}\right] \\
\text { using SC's preamble. }
\end{array}\right.
$$

From (A.23), (A.26), (A.31), (A.34), (A.35) and (A.40), we have

$$
\begin{aligned}
& E\left\{\left|P_{d}\right|^{2}\right\}=E\left\{\left(\left|d_{0}\right|^{2}-\left|d_{L_{p}}\right|^{2}\right)^{2}\right\}+E\left\{2\left(\left|d_{0}\right|^{2}-\left|d_{L_{p}}\right|^{2}\right) \cdot 2 \Re\left\{\left(d_{0}-d_{L_{p}}\right) \sum_{m=1}^{L_{p}-1} d_{m}^{*}\right\}\right\} \\
& +E\left\{\left[\left(d_{0}^{*}-d_{L_{p}}^{*}\right) \sum_{m=1}^{L_{p}-1} d_{m}\right]^{2}\right\}+E\left\{2\left|\left(d_{0}^{*}-d_{L_{p}}^{*}\right) \sum_{m=1}^{L_{p}-1} d_{m}\right|^{2}\right\} \\
& +E\left\{\left[\left(d_{0}-d_{L_{p}}\right) \sum_{m=1}^{L_{p}-1} d_{m}^{*}\right]^{2}\right\} \\
& \left\{\begin{array}{l}
8 \sigma_{s}^{6} \sigma_{n}^{2}+8 \sigma_{s}^{4} \sigma_{n}^{4}+8 \sigma_{s}^{2} \sigma_{n}^{6}+4\left(2 \sigma_{s}^{4}+\sigma_{s}^{2} \sigma_{n}^{2}\right) \sigma_{s}^{4}\left(L_{p}-1\right)^{2} \\
+4\left(2 \sigma_{s}^{4}+2 \sigma_{s}^{2} \sigma_{n}^{2}+\sigma_{n}^{4}\right)\left[\left(L_{p}-1\right)\left(\sigma_{s}^{4}+2 \sigma_{s}^{2} \sigma_{n}^{2}+\sigma_{n}^{4}\right)+\left(L_{p}-1\right)\left(L_{p}-2\right) \sigma_{s}^{4}\right]
\end{array}\right. \\
& \text { using the FIP preamble. } \\
& =\{ \\
& 8 \sigma_{s}^{6} \sigma_{n}^{2}+8 \sigma_{s}^{4} \sigma_{n}^{4}+8 \sigma_{s}^{2} \sigma_{n}^{6}+4\left(L_{p}-1\right) \sigma_{s}^{4}\left(\sigma_{s}^{2} \sigma_{n}^{2}+2 \sigma_{n}^{4}\right)+2 \sigma_{s}^{8}\left(L_{p}-1\right)^{2} \\
& +4\left(\sigma_{s}^{4}+2 \sigma_{s}^{2} \sigma_{n}^{2}+\sigma_{n}^{4}\right)\left[\left(L_{p}-1\right)\left(\sigma_{s}^{4}+2 \sigma_{s}^{2} \sigma_{n}^{2}+\sigma_{n}^{4}\right)+\left(L_{p}-1\right)\left(L_{p}-2\right) \sigma_{s}^{4}\right] \\
& \text { using SC's preamble. }
\end{aligned}
$$


From (A.22) and (A.41), we have

$$
\begin{aligned}
& \operatorname{Var}\left(P_{d}\right)=E\left\{\left|P_{d}\right|^{2}\right\}-\left(E\left\{P_{d}\right\}\right)^{2} \\
& \left\{\begin{array}{l}
8 \sigma_{s}^{6} \sigma_{n}^{2}+8 \sigma_{s}^{4} \sigma_{n}^{4}+8 \sigma_{s}^{2} \sigma_{n}^{6}+4\left(2 \sigma_{s}^{4}+\sigma_{s}^{2} \sigma_{n}^{2}\right) \sigma_{s}^{4}\left(L_{p}-1\right)^{2} \\
+4\left(2 \sigma_{s}^{4}+2 \sigma_{s}^{2} \sigma_{n}^{2}+\sigma_{n}^{4}\right)\left[\left(L_{p}-1\right)\left(\sigma_{s}^{4}+2 \sigma_{s}^{2} \sigma_{n}^{2}+\sigma_{n}^{4}\right)+\left(L_{p}-1\right)\left(L_{p}-2\right) \sigma_{s}^{4}\right] \\
-\left[4\left(L_{p}-1\right) \sigma_{s}^{4}\right]^{2}
\end{array}\right. \\
& \text { using the FlP preamble. } \\
& = \\
& 8 \sigma_{s}^{6} \sigma_{n}^{2}+8 \sigma_{s}^{4} \sigma_{n}^{4}+8 \sigma_{s}^{2} \sigma_{n}^{6}+4\left(L_{p}-1\right) \sigma_{s}^{4}\left(\sigma_{s}^{2} \sigma_{n}^{2}+2 \sigma_{n}^{4}\right)+2 \sigma_{s}^{8}\left(L_{p}-1\right)^{2} \\
& +4\left(\sigma_{s}^{4}+2 \sigma_{s}^{2} \sigma_{n}^{2}+\sigma_{n}^{4}\right)\left[\left(L_{p}-1\right)\left(\sigma_{s}^{4}+2 \sigma_{s}^{2} \sigma_{n}^{2}+\sigma_{n}^{4}\right)+\left(L_{p}-1\right)\left(L_{p}-2\right) \sigma_{s}^{4}\right] \\
& -\left[2\left(L_{p}-1\right) \sigma_{s}^{4}\right]^{2} \\
& \text { using SC's preamble. } \\
& \left\{\begin{array}{l}
4\left(L_{p}-1\right)^{2}\left(3 \sigma_{s}^{6} \sigma_{n}^{2}+\sigma_{s}^{4} \sigma_{n}^{4}\right)+4\left(L_{p}-1\right)\left(2 \sigma_{s}^{4}+2 \sigma_{s}^{2} \sigma_{n}^{2}+\sigma_{n}^{4}\right) \\
\left(2 \sigma_{s}^{2} \sigma_{n}^{2}+\sigma_{n}^{4}\right)+8\left(\sigma_{s}^{6} \sigma_{n}^{2}+\sigma_{s}^{4} \sigma_{n}^{4}+\sigma_{s}^{2} \sigma_{n}^{6}\right)
\end{array}\right. \\
& \text { using the FlP preamble. } \\
& =\{ \\
& \left(L_{p}-1\right)^{2} \sigma_{s}^{4}\left(2 \sigma_{s}^{4}+8 \sigma_{s}^{2} \sigma_{n}^{2}+4 \sigma_{n}^{4}\right)+4\left(L_{p}-1\right)\left[3 \sigma_{s}^{4}\left(\sigma_{s}^{2} \sigma_{n}^{2}+\sigma_{n}^{4}\right)\right. \\
& \left.+\left(2 \sigma_{s}^{2} \sigma_{n}^{2}+\sigma_{n}^{4}\right)^{2}\right]+8\left(\sigma_{s}^{6} \sigma_{n}^{2}+\sigma_{s}^{4} \sigma_{n}^{4}+\sigma_{s}^{2} \sigma_{n}^{6}\right) \\
& \text { using SC's preamble. }
\end{aligned}
$$

Secondly, let us calculate the mean and variance of $R_{d}$. Let us define

$$
q_{m}=\left|s_{d_{o p t}+m+L_{p}}\right|^{2}+s_{d_{o p t}+m+L_{p}}^{*} n_{d_{o p t}+m+L_{p}}+s_{d_{o p t}+m+L_{p}} n_{d_{o p t}+m+L_{p}}^{*}+\left|n_{d_{o p t}+m+L_{p}}\right|^{2}
$$


Recall $R_{d}=R_{p}^{2}\left(d_{o p t+1}\right)-R_{p}^{2}\left(d_{o p t}\right)$ and from (A.10), we have

$$
\begin{gathered}
R_{d}=R_{p}^{2}\left(d_{o p t+1}\right)-R_{p}^{2}\left(d_{o p t}\right)=\left(\sum_{m=1}^{L_{p}} q_{m}\right)^{2}-\left(\sum_{m=0}^{L_{p}-1} q_{m}\right)^{2} \\
=\left(q_{L_{p}}+\sum_{m=1}^{L_{p}-1} q_{m}\right)^{2}-\left(q_{0}+\sum_{m=1}^{L_{p}-1} q_{m}\right)^{2} \\
=q_{L_{p}}^{2}+2 q_{L_{p}} \sum_{m=1}^{L_{p}-1} q_{m}+\left(\sum_{m=1}^{L_{p}-1} q_{m}\right)^{2}-q_{0}^{2}-2 q_{0} \sum_{m=1}^{L_{p}-1} q_{m}-\left(\sum_{m=1}^{L_{p}-1} q_{m}\right)^{2}(\mathrm{~A} .44) \\
=\left(q_{L_{p}}^{2}-q_{0}^{2}\right)+2\left(q_{L_{p}}-q_{0}\right) \sum_{m=1}^{L_{p}-1} q_{m} \\
q_{m}^{2}=\left(\left|s_{d_{o p t}+m+L_{p}}\right|^{2}+s_{d_{o p t}+m+L_{p}}^{*} n_{d_{o p t}+m+L_{p}}+s_{d_{o p t}+m+L_{p}} n_{d_{o p t}+m+L_{p}}^{*}+\left|n_{d_{o p t}+m+L_{p}}\right|^{2}\right)^{2} \\
=\left|s_{d_{o p t}+m+L_{p}}\right|^{4}+\left(s_{d_{o p t}+m+L_{p}}^{*} n_{d_{o p t}+m+L_{p}}\right)^{2}+\left(s_{d_{o p t}+m+L_{p}} n_{d_{o p t}+m+L_{p}}^{*}+\left|n_{d_{o p t}+m+L_{p}}\right|^{4}\right. \\
+2\left|s_{d_{o p t}+m+L_{p}}\right|^{2} s_{d_{o p t}+m+L_{p}}^{*} n_{d_{o p t}+m+L_{p}}+2\left|s_{d_{o p t}+m+L_{p}}\right|^{2} s_{d_{o p t}+m+L_{p}} n_{d_{o p t}+m+L_{p}}^{*} \\
+2\left|s_{d_{o p t}+m+L_{p}}\right|^{2}\left|n_{d_{o p t}+m+L_{p}}\right|^{2}+2\left|s_{d_{o p t}+m+L_{p}}\right|^{2}\left|n_{d_{o p t}+m+L_{p}}\right|^{2} \\
+2 s_{d_{o p t}+m+L_{p}}^{*} n_{d_{o p t}+m+L_{p}}\left|n_{d_{o p t}+m+L_{p}}\right|^{2}+2 s_{d_{o p t}+m+L_{p}} n_{d_{o p t}+m+L_{p}}^{*}\left|n_{d_{o p t}+m+L_{p}}\right|^{2}
\end{gathered}
$$

We have

$$
\begin{aligned}
& E\left\{q_{m}^{2}\right\}=\sigma_{s}^{4}+4 \sigma_{s}^{2} \sigma_{n}^{2}+\sigma_{n}^{4} \\
& E\left\{q_{m}\right\}=\sigma_{s}^{2}+\sigma_{n}^{2}
\end{aligned}
$$

Since $q_{m}$ are independent with each other, we have

$$
\begin{aligned}
E\left\{R_{d}\right\} & =E\left\{\left(q_{L_{p}}^{2}-q_{0}^{2}\right)+2\left(q_{L_{p}}-q_{0}\right) \sum_{m=1}^{L_{p}-1} q_{m}\right\} \\
& =E\left\{q_{L_{p}}^{2}\right\}-E\left\{q_{0}^{2}\right\}+2\left(E\left\{q_{L_{p}}\right\}-E\left\{q_{0}\right\}\right) E\left\{\sum_{m=1}^{L_{p}-1} q_{m}\right\}=0
\end{aligned}
$$




$$
\begin{aligned}
R_{d}^{2} & =\left[\left(q_{L_{p}}^{2}-q_{0}^{2}\right)+2\left(q_{L_{p}}-q_{0}\right) \sum_{m=1}^{L_{p}-1} q_{m}\right]^{2} \\
& =\left(q_{L_{p}}^{2}-q_{0}^{2}\right)^{2}+4\left(q_{L_{p}}^{2}-q_{0}^{2}\right)\left(q_{L_{p}}-q_{0}\right) \sum_{m=1}^{L_{p}-1} q_{m}+4\left(q_{L_{p}}-q_{0}\right)^{2}\left(\sum_{m=1}^{L_{p}-1} q_{m}\right)^{2}
\end{aligned}
$$

$$
\begin{aligned}
& E\left\{q_{m}^{4}\right\}=E\left\{\left[\left|s_{d_{o p t}+m+L_{p}}\right|^{4}+4\left|s_{d_{o p t}+m+L_{p}}\right|^{2}\left|n_{d_{o p t}+m+L_{p}}\right|^{2}+\left|n_{d_{o p t}+m+L_{p}}\right|^{4}\right.\right. \\
& +2\left(\left|s_{d_{o p t}+m+L_{p}}\right|^{2}+\left|n_{d_{o p t}+m+L_{p}}\right|^{2}\right)\left(s_{d_{o p t}+m+L_{p}}^{*} n_{d_{o p t}+m+L_{p}}+s_{d_{o p t}+m+L_{p}} n_{d_{o p t}+m+L_{p}}^{*}\right) \\
& \left.\left.+\left(s_{d_{o p t}+m+L_{p}}^{*} n_{d_{o p t}+m+L_{p}}\right)^{2}+\left(s_{d_{o p t}+m+L_{p}} n_{d_{o p t}+m+L_{p}}^{*}\right)^{2}\right]^{2}\right\} \\
& \quad=\left(\sigma_{s}^{4}+4 \sigma_{s}^{2} \sigma_{n}^{2}+\sigma_{n}^{4}\right)^{2}+8 \sigma_{s}^{2} \sigma_{n}^{2}\left(\sigma_{s}^{2}+\sigma_{n}^{2}\right)^{2}+2 \sigma_{s}^{4} \sigma_{n}^{4}
\end{aligned}
$$

$$
\begin{aligned}
E\left\{q_{0}^{2} q_{L_{p}}^{2}\right\}= & \left\{\left[\left|s_{d_{o p t}+L_{p}}\right|^{4}+4\left|s_{d_{o p t}+L_{p}}\right|^{2}\left|n_{d_{o p t}+L_{p}}\right|^{2}+\left|n_{d_{o p t}+L_{p}}\right|^{4}\right.\right. \\
& +2\left(\left|s_{d_{o p t}+L_{p}}\right|^{2}+\left|n_{d_{o p t}+L_{p}}\right|^{2}\right)\left(s_{d_{o p t}+L_{p}}^{*} n_{d_{o p t}+L_{p}}+s_{d_{o p t}+L_{p}} n_{d_{o p t}+L_{p}}^{*}\right) \\
& \left.+\left(s_{d_{o p t}+L_{p}}^{*} n_{d_{o p t}+L_{p}}\right)^{2}+\left(s_{d_{o p t}+L_{p}} n_{d_{o p t}+L_{p}}^{*}\right)^{2}\right] \\
\cdot & {\left[\left|s_{d_{o p t}+2 L_{p}}\right|^{4}+4\left|s_{d_{o p t}+2 L_{p}}\right|^{2}\left|n_{d_{o p t}+2 L_{p}}\right|^{2}+\left|n_{d_{o p t}+2 L_{p}}\right|^{4}\right.} \\
& +2\left(\left|s_{d_{o p t}+2 L_{p}}\right|^{2}+\left|n_{d_{o p t}+2 L_{p}}\right|^{2}\right)\left(s_{d_{o p t}+2 L_{p}}^{*} n_{d_{o p t}+2 L_{p}}+s_{d_{o p t}+2 L_{p}} n_{d_{o p t}+2 L_{p}}^{*}\right) \\
& \left.\left.+\left(s_{d_{o p t}+2 L_{p}}^{*} n_{d_{o p t}+2 L_{p}}\right)^{2}+\left(s_{d_{o p t}+2 L_{p}} n_{d_{o p t}+2 L_{p}}^{*}\right)^{2}\right]\right\} \\
= & \left(\sigma_{s}^{4}+4 \sigma_{s}^{2} \sigma_{n}^{2}+\sigma_{n}^{4}\right)^{2}
\end{aligned}
$$

$$
E\left\{\left(q_{L_{p}}^{2}-q_{0}^{2}\right)^{2}\right\}=E\left\{q_{L_{p}}^{4}-2 q_{0}^{2} q_{L_{p}}^{2}+q_{0}^{4}\right\}=16 \sigma_{s}^{2} \sigma_{n}^{2}\left(\sigma_{s}^{2}+\sigma_{n}^{2}\right)^{2}+4 \sigma_{s}^{4} \sigma_{n}^{4}
$$




$$
\begin{gathered}
E\left\{q_{m}^{3}\right\}=E\left\{\left(\left|s_{d_{o p t}+m+L_{p}}\right|^{4}+4\left|s_{d_{o p t}+m+L_{p}}\right|^{2}\left|n_{d_{o p t}+m+L_{p}}\right|^{2}+\left|n_{d_{o p t}+m+L_{p}}\right|^{4}\right.\right. \\
+2\left(\left|s_{d_{o p t}+m+L_{p}}\right|^{2}+\left|n_{d_{o p t}+m+L_{p}}\right|^{2}\right)\left(s_{d_{o p t}+m+L_{p}}^{*} n_{d_{o p t}+m+L_{p}}+s_{d_{o p t}+m+L_{p}} n_{d_{o p t}+m+L_{p}}^{*}\right) \\
\left.+\left(s_{d_{o p t}+m+L_{p}}^{*} n_{d_{o p t}+m+L_{p}}\right)^{2}+\left(s_{d_{o p t}+m+L_{p}} n_{d_{o p t}+m+L_{p}}^{*}\right)^{2}\right) \\
\left.\cdot\left(\left|s_{d_{o p t}+m+L_{p}}\right|^{2}+\left|n_{d_{o p t}+m+L_{p}}\right|^{2}+s_{d_{o p t}+m+L_{p}}^{*} n_{d_{o p t}+m+L_{p}}+s_{d_{o p t}+m+L_{p}} n_{d_{o p t}+m+L_{p}}^{*}\right)\right\} \\
=\left(\sigma_{s}^{4}+8 \sigma_{s}^{2} \sigma_{n}^{2}+\sigma_{n}^{4}\right)\left(\sigma_{s}^{2}+\sigma_{n}^{2}\right) \\
\begin{array}{c}
E\left\{q_{0}^{2} q_{L_{p}}\right\}=E \\
\quad\left\{\left(\left|s_{d_{o p t}+L_{p}}\right|^{4}+4\left|s_{d_{o p t}+L_{p}}\right|^{2}\left|n_{d_{o p t}+L_{p}}\right|^{2}+\left|n_{d_{o p t}+L_{p}}\right|^{4}\right.\right. \\
+2\left(\left|s_{d_{o p t}+L_{p}}\right|^{2}+\left|n_{d_{o p t}+L_{p}}\right|^{2}\right)\left(s_{d_{o p t}+L_{p}}^{*} n_{d_{o p t}+L_{p}}+s_{d_{o p t}+L_{p}} n_{d_{o p t}+L_{p}}^{*}\right) \\
\left.\quad+\left(s_{d_{o p t}+L_{p}}^{*} n_{d_{o p t}+L_{p}}\right)^{2}+\left(s_{d_{o p t}+L_{p}} n_{d_{o p t}+L_{p}}^{*}\right)^{2}\right) \\
\left.\cdot\left(\left|s_{d_{o p t}+2 L_{p}}\right|^{2}+\left|n_{d_{o p t}+2 L_{p}}\right|^{2}+s_{d_{o p t}+2 L_{p}}^{*} n_{d_{o p t}+2 L_{p}}+s_{d_{o p t}+2 L_{p}} n_{d_{o p t}+2 L_{p}}^{*}\right)\right\} \\
=\left(\sigma_{s}^{4}+4 \sigma_{s}^{2} \sigma_{n}^{2}+\sigma_{n}^{4}\right)\left(\sigma_{s}^{2}+\sigma_{n}^{2}\right)
\end{array}
\end{gathered}
$$

Since $q_{m}$ are independent, we have

$$
\begin{aligned}
E\left\{\left(q_{L_{p}}^{2}-q_{0}^{2}\right)\left(q_{L_{p}}-q_{0}\right) \sum_{m=1}^{L_{p}-1} q_{m}\right\} & =E\left\{\left(q_{L_{p}}^{2}-q_{0}^{2}\right)\left(q_{L_{p}}-q_{0}\right)\right\} E\left\{\sum_{m=1}^{L_{p}-1} q_{m}\right\} \\
& =\left(L_{p}-1\right)\left(\sigma_{s}^{2}+\sigma_{n}^{2}\right) E\left\{q_{L_{p}}^{3}+q_{0}^{3}-q_{L_{p}}^{2} q_{0}-q_{0}^{2} q_{L_{p}}\right\} \\
& =8\left(L_{p}-1\right) \sigma_{s}^{2} \sigma_{n}^{2}\left(\sigma_{s}^{2}+\sigma_{n}^{2}\right)^{2}
\end{aligned}
$$




$$
\begin{aligned}
E\left\{q_{0} q_{L_{p}}\right\}= & E\left\{\left(\left|s_{d_{o p t}+L_{p}}\right|^{2}+\left|n_{d_{o p t}+L_{p}}\right|^{2}+s_{d_{o p t}+L_{p}}^{*} n_{d_{o p t}+L_{p}}+s_{d_{o p t}+L_{p}} n_{d_{o p t}+L_{p}}^{*}\right)\right. \\
& \left.\cdot\left(\left|s_{d_{o p t}+2 L_{p}}\right|^{2}+\left|n_{d_{o p t}+2 L_{p}}\right|^{2}+s_{d_{o p t}+2 L_{p}}^{*} n_{d_{o p t}+2 L_{p}}+s_{d_{o p t}+2 L_{p}} n_{d_{o p t}+2 L_{p}}^{*}\right)\right\} \\
= & \left(\sigma_{s}^{2}+\sigma_{n}^{2}\right)^{2}
\end{aligned}
$$

From (A.46) and (A.58)

$$
\begin{aligned}
& E\left\{\left(q_{L_{p}}-q_{0}\right)^{2}\right\}=E\left\{q_{L_{p}}^{2}-2 q_{L_{p}} q_{0}+q_{0}^{2}\right\}=E\left\{q_{L_{p}}^{2}\right\}-2 E\left\{q_{L_{p}} q_{0}\right\}+E\left\{q_{0}^{2}\right\}=4 \sigma_{s}^{2} \sigma_{n}^{2} \\
& E\left\{\left(\sum_{m=1}^{L_{p}-1} q_{m}\right)^{2}\right\}=E\left\{\sum_{m=1}^{L_{p}-1} q_{m}^{2}+\sum_{m=1}^{L_{p}-1} \sum_{n=1, n \neq m}^{L_{p}-1} q_{m} q_{n}\right\} \\
& =E\left\{\sum_{m=1}^{L_{p}-1} q_{m}^{2}\right\}+E\left\{\sum_{m=1}^{L_{p}-1} \sum_{n=1, n \neq m}^{L_{p}-1} q_{m} q_{n}\right\} \\
& =\left(L_{p}-1\right)\left(\sigma_{s}^{4}+4 \sigma_{s}^{2} \sigma_{n}^{2}+\sigma_{n}^{4}\right)+\sum_{m=1}^{L_{p}-1} \sum_{n=1, n \neq m}^{L_{p}-1} E\left\{q_{m}\right\} E\left\{q_{n}\right\} \\
& =2\left(L_{p}-1\right) \sigma_{s}^{2} \sigma_{n}^{2}+\left(L_{p}-1\right)^{2}\left(\sigma_{s}^{2}+\sigma_{n}^{2}\right)^{2} \\
& E\left\{\left(q_{L_{p}}-q_{0}\right)^{2}\left(\sum_{m=1}^{L_{p}-1} q_{m}\right)^{2}\right\}=E\left\{\left(q_{L_{p}}-q_{0}\right)^{2}\right\} E\left\{\left(\sum_{m=1}^{L_{p}-1} q_{m}\right)^{2}\right\} \\
& =4 \sigma_{s}^{2} \sigma_{n}^{2}\left[2\left(L_{p}-1\right) \sigma_{s}^{2} \sigma_{n}^{2}+\left(L_{p}-1\right)^{2}\left(\sigma_{s}^{2}+\sigma_{n}^{2}\right)^{2}\right]
\end{aligned}
$$


From (A.48), (A.52), (A.57) and (A.61)

$$
\begin{aligned}
& \operatorname{Var}\left(R_{d}\right)=E\left\{R_{d}^{2}\right\}-\left(E\left\{R_{d}\right\}\right)^{2} \\
& =E\left\{\left(q_{L_{p}}^{2}-q_{0}^{2}\right)^{2}+4\left(q_{L_{p}}^{2}-q_{0}^{2}\right)\left(q_{L_{p}}-q_{0}\right) \sum_{m=1}^{L_{p}-1} q_{m}+4\left(q_{L_{p}}-q_{0}\right)^{2}\left(\sum_{m=1}^{L_{p}-1} q_{m}\right)^{2}\right\}-0 \\
& =E\left\{\left(q_{L_{p}}^{2}-q_{0}^{2}\right)^{2}\right\}+4 E\left\{\left(q_{L_{p}}^{2}-q_{0}^{2}\right)\left(q_{L_{p}}-q_{0}\right) \sum_{m=1}^{L_{p}-1} q_{m}\right\} \\
& \quad+4 E\left\{\left(q_{L_{p}}-q_{0}\right)^{2}\left(\sum_{m=1}^{L_{p}-1} q_{m}\right)^{2}\right\} \\
& =16 \sigma_{s}^{2} \sigma_{n}^{2}\left(\sigma_{s}^{2}+\sigma_{n}^{2}\right)^{2}+4 \sigma_{s}^{4} \sigma_{n}^{4}+32\left(L_{p}-1\right) \sigma_{s}^{2} \sigma_{n}^{2}\left(\sigma_{s}^{2}+\sigma_{n}^{2}\right)^{2} \\
& +16 \sigma_{s}^{2} \sigma_{n}^{2}\left[2\left(L_{p}-1\right) \sigma_{s}^{2} \sigma_{n}^{2}+\left(L_{p}-1\right)^{2}\left(\sigma_{s}^{2}+\sigma_{n}^{2}\right)^{2}\right] \\
& =16 L_{p}^{2} \sigma_{s}^{2} \sigma_{n}^{2}\left(\sigma_{s}^{2}+\sigma_{n}^{2}\right)^{2}+\left(32 L_{p}-28\right) \sigma_{s}^{4} \sigma_{n}^{4}
\end{aligned}
$$

Thirdly, let us calculate the covariance of $P_{d}$ and $R_{d}$ (both are real)

$$
\begin{aligned}
& \operatorname{Cov}\left(P_{d}, R_{d}\right)=E\left\{\left(P_{d}-E\left\{P_{d}\right\}\right)^{*}\left(R_{d}-E\left\{R_{d}\right\}\right)\right\} \\
& =E\left\{P_{d} R_{d}-E\left\{P_{d}\right\} R_{d}-0\left(P_{d}-E\left\{P_{d}\right\}\right)\right\} \\
& =E\left\{P_{d} R_{d}\right\}-E\left\{P_{d}\right\} E\left\{R_{d}\right\}=E\left\{P_{d} R_{d}\right\} \\
& =E\left\{\left[\left|d_{0}\right|^{2}-\left|d_{L_{p}}\right|^{2}+2 \Re\left\{\left(d_{0}^{*}-d_{L_{p}}^{*}\right) \sum_{m=1}^{L_{p}-1} d_{m}\right\}\right]\left[\left(q_{L_{p}}^{2}-q_{0}^{2}\right)+2\left(q_{L_{p}}-q_{0}\right) \sum_{n=1}^{L_{p}-1} q_{n}\right]\right\} \\
& =E\left\{\left(\left|d_{0}\right|^{2}-\left|d_{L_{p}}\right|^{2}\right)\left(q_{L_{p}}^{2}-q_{0}^{2}\right)\right\}+4 E\left\{\Re\left\{\left(d_{0}^{*}-d_{L_{p}}^{*}\right) \sum_{m=1}^{L_{p}-1} d_{m}\right\}\left(q_{L_{p}}-q_{0}\right) \sum_{n=1}^{L_{p}-1} q_{n}\right\} \\
& +2 E\left\{\left(q_{L_{p}}^{2}-q_{0}^{2}\right) \Re\left\{\left(d_{0}^{*}-d_{L_{p}}^{*}\right) \sum_{m=1}^{L_{p}-1} d_{m}\right\}\right\}+2 E\left\{\left(\left|d_{0}\right|^{2}-\left|d_{L_{p}}\right|^{2}\right)\left(q_{L_{p}}-q_{0}\right) \sum_{n=1}^{L_{p}-1} q_{n}\right\}
\end{aligned}
$$




$$
\begin{aligned}
E\{\mid & \left.\left.d_{m}\right|^{2} q_{m}^{2}\right\}=E\left\{\left(\left|s_{d_{o p t}+m}\right|^{2}\left|s_{d_{o p t}+m+L_{p}}\right|^{2}+\left|s_{d_{o p t}+m}\right|^{2} n_{d_{o p t}+m+L_{p}}^{*} s_{d_{o p t}+m+L_{p}}\right.\right. \\
& +\left|s_{d_{o p t}+m+L_{p}}\right|^{2} s_{d_{o p t}+m}^{*} n_{d_{o p t}+m}+n_{d_{o p t}+m} n_{d_{o p t}+m+L_{p}}^{*} s_{d_{o p t}+m}^{*} s_{d_{o p t}+m+L_{p}} \\
& +\left|s_{d_{o p t}+m}\right|^{2} s_{d_{o p t}+m+L_{p}}^{*} n_{d_{o p t}+m+L_{p}}+\left|s_{d_{o p t}+m}\right|^{2}\left|n_{d_{o p t}+m+L_{p}}\right|^{2} \\
& +s_{d_{o p t}+m+L_{p}}^{*} n_{d_{o p t}+m} s_{d_{o p t}+m}^{*} n_{d_{o p t}+m+L_{p}}+n_{d_{o p t}+m} s_{d_{o p t}+m}^{*}\left|n_{d_{o p t}+m+L_{p}}\right|^{2} \\
& +s_{d_{o p t}+m}\left|s_{d_{o p t}+m+L_{p}}\right|^{2} n_{d_{o p t}+m}^{*}+s_{d_{o p t}+m} n_{d_{o p t}+m+L_{p}}^{*} s_{d_{o p t}+m+L_{p}} n_{d_{o p t}+m}^{*} \\
& +\left|s_{d_{o p t}+m+L_{p}}\right|^{2}\left|n_{d_{o p t}+m}\right|^{2}+\left|n_{d_{o p t}+m}\right|^{2} n_{d_{o p t}+m+L_{p}}^{*} s_{d_{o p t}+m+L_{p}} \\
& +s_{d_{o p t}+m} s_{d_{o p t}+m+L_{p}}^{*} n_{d_{o p t}+m}^{*} n_{d_{o p t}+m+L_{p}}+s_{d_{o p t}+m} n_{d_{o p t}+m}^{*}\left|n_{d_{o p t}+m+L_{p}}\right|^{2} \\
& \left.+s_{d_{o p t}+m+L_{p}}^{*}\left|n_{d_{o p t}+m}\right|^{2} n_{d_{o p t}+m+L_{p}}+\left|n_{d_{o p t}+m}\right|^{2}\left|n_{d_{o p t}+m+L_{p}}\right|^{2}\right) \\
& +\left(\left|s_{d_{o p t}+m+L_{p}}\right|^{4}+4\left|s_{d_{o p t}+m+L_{p}}\right|^{2}\left|n_{d_{o p t}+m+L_{p}}\right|^{2}+\left|n_{d_{o p t}+m+L_{p}}\right|^{4}\right. \\
& +2\left(\left|s_{d_{o p t}+m+L_{p}}\right|^{2}+\left|n_{d_{o p t}+m+L_{p}}\right|^{2}\right)\left(s_{d_{o p t}+m+L_{p}}^{*} n_{d_{o p t}+m+L_{p}}+s_{d_{o p t}+m+L_{p}} n_{d_{o p t}+m+L_{p}}^{*}\right) \\
& \left.\left.+\left(s_{d_{o p t}+m+L_{p}}^{*} n_{d_{o p t}+m+L_{p}}\right)^{2}+\left(s_{d_{o p t}+m+L_{p}} n_{d_{o p t}+m+L_{p}}^{*}\right)^{2}\right)\right\} \\
=( & \left.\sigma_{s}^{4}+2 \sigma_{s}^{2} \sigma_{n}^{2}+\sigma_{n}^{4}\right)\left(\sigma_{s}^{4}+4 \sigma_{s}^{2} \sigma_{n}^{2}+\sigma_{n}^{4}\right) \\
+ & 2\left(\sigma_{s}^{2}+\sigma_{n}^{2}\right)\left(\sigma_{s}^{4} \sigma_{n}^{2}+\sigma_{s}^{4} \sigma_{n}^{2}+\sigma_{s}^{2} \sigma_{n}^{4}+\sigma_{s}^{2} \sigma_{n}^{4}\right)+0+0 \\
= & \left.+\sigma_{n}^{2}\right)^{2}\left(\sigma_{s}^{4}+8 \sigma_{s}^{2} \sigma_{n}^{2}+\sigma_{n}^{4}\right)
\end{aligned}
$$




$$
\begin{aligned}
& E\left\{\left|d_{0}\right|^{2} q_{L_{p}}^{2}\right\}=E\left\{\left(\left|s_{d_{o p t}}\right|^{2}\left|s_{d_{o p t}+L_{p}}\right|^{2}+\left|s_{d_{o p t}}\right|^{2} n_{d_{o p t}+L_{p}}^{*} s_{d_{o p t}+L_{p}}\right.\right. \\
&+\left|s_{d_{o p t}+L_{p}}\right|^{2} s_{d_{o p t}}^{*} n_{d_{o p t}}+n_{d_{o p t}} n_{d_{o p t}+L_{p}}^{*} s_{d_{o p t}}^{*} s_{d_{o p t}+L_{p}} \\
&+\left|s_{d_{o p t}}\right|^{2} s_{d_{o p t}+L_{p}}^{*} n_{d_{o p t}+L_{p}}+\left|s_{d_{o p t}}\right|^{2}\left|n_{d_{o p t}+L_{p}}\right|^{2} \\
&+s_{d_{o p t}+L_{p}}^{*} n_{d_{o p t}} s_{d_{o p t}}^{*} n_{d_{o p t}+L_{p}}+n_{d_{o p t}} s_{d_{o p t}}^{*}\left|n_{d_{o p t}+L_{p}}\right|^{2} \\
&+s_{d_{o p t}}\left|s_{d_{o p t}+L_{p}}\right|^{2} n_{d_{o p t}}^{*}+s_{d_{o p t}} n_{d_{o p t}+L_{p}}^{*} s_{d_{o p t}+L_{p}} n_{d_{o p t}}^{*} \\
&+\left|s_{d_{o p t}}+L_{p}\right|^{2}\left|n_{d_{o p t}}\right|^{2}+\left|n_{d_{o p t}}\right|^{2} n_{d_{o p t}+L_{p}}^{*} s_{d_{o p t}+L_{p}} \\
&+s_{d_{o p t}} s_{d_{o p t}+L_{p}}^{*} n_{d_{o p t}}^{*} n_{d_{o p t}+L_{p}}+s_{d_{o p t}} n_{d_{o p t}}^{*}\left|n_{d_{o p t}+L_{p}}\right|^{2} \\
&\left.+s_{d_{o p t}+L_{p}}^{*}\left|n_{d_{o p t}}\right|^{2} n_{d_{o p t}+L_{p}}+\left|n_{d_{o p t}}\right|^{2}\left|n_{d_{o p t}+L_{p}}\right|^{2}\right) \\
&+\left[\left|s_{d_{o p t}+2 L_{p}}\right|^{4}+4\left|s_{d_{o p t}+2 L_{p}}\right|^{2}\left|n_{d_{o p t}+2 L_{p}}\right|^{2}+\left|n_{d_{o p t}+2 L_{p}}\right|^{4}\right. \\
&+2\left(\left|s_{d_{o p t}+2 L_{p}}\right|^{2}+\left|n_{d_{o p t}+2 L_{p}}\right|^{2}\right)\left(s_{d_{o p t}+2 L_{p}}^{*} n_{d_{o p t}+2 L_{p}}+s_{d_{o p t}+2 L_{p}} n_{d_{o p t}+2 L_{p}}^{*}\right) \\
&\left.\left.+\left(s_{d_{o p t}+2 L_{p}}^{*} n_{d_{o p t}+2 L_{p}}\right)^{2}+\left(s_{d_{o p t}+2 L_{p}} n_{d_{o p t}+2 L_{p}}^{*}\right)^{2}\right]\right\} \\
&=(\left.\sigma_{s}^{4}+2 \sigma_{s}^{2} \sigma_{n}^{2}+\sigma_{n}^{4}\right)\left(\sigma_{s}^{4}+4 \sigma_{s}^{2} \sigma_{n}^{2}+\sigma_{n}^{4}\right)+0+0+0 \\
&=\left(\sigma_{s}^{2}+\sigma_{n}^{2}\right)^{2}\left(\sigma_{s}^{4}+4 \sigma_{s}^{2} \sigma_{n}^{2}+\sigma_{n}^{4}\right)
\end{aligned}
$$

Similarly, we have

$$
\begin{gathered}
E\left\{\left|d_{L_{p}}\right|^{2} q_{0}^{2}\right\}=E\left\{\left|d_{0}\right|^{2} q_{L_{p}}^{2}\right\}=\left(\sigma_{s}^{2}+\sigma_{n}^{2}\right)^{2}\left(\sigma_{s}^{4}+4 \sigma_{s}^{2} \sigma_{n}^{2}+\sigma_{n}^{4}\right) \\
E\left\{\left(\left|d_{0}\right|^{2}-\left|d_{L_{p}}\right|^{2}\right)\left(q_{L_{p}}^{2}-q_{0}^{2}\right)\right\}=E\left\{\left|d_{0}\right|^{2} q_{L_{p}}^{2}+\left|d_{L_{p}}\right|^{2} q_{0}^{2}-\left|d_{L_{p}}\right|^{2} q_{L_{p}}^{2}-\left|d_{0}\right|^{2} q_{0}^{2}\right\} \\
=E\left\{\left|d_{0}\right|^{2} q_{L_{p}}^{2}\right\}+E\left\{\left|d_{L_{p}}\right|^{2} q_{0}^{2}\right\}-E\left\{\left|d_{L_{p}}\right|^{2} q_{L_{p}}^{2}\right\}-E\left\{\left|d_{0}\right|^{2} q_{0}^{2}\right\} \\
=-8 \sigma_{s}^{2} \sigma_{n}^{2}\left(\sigma_{s}^{2}+\sigma_{n}^{2}\right)^{2}
\end{gathered}
$$




$$
\begin{aligned}
E\left\{d_{0}^{*} q_{L_{p}}\right\}= & E\left\{\left(s_{d_{o p t}} s_{d_{o p t}+L_{p}}^{*}+s_{d_{o p t}} n_{d_{o p t}+L_{p}}^{*}+s_{d_{o p t}+L_{p}}^{*} n_{d_{o p t}}+n_{d_{o p t}} n_{d_{o p t}+L_{p}}^{*}\right)\right. \\
& \left.\cdot\left(\left|s_{d_{o p t}+2 L_{p}}\right|^{2}+s_{d_{o p t}+2 L_{p}}^{*} n_{d_{o p t}+2 L_{p}}+s_{d_{o p t}+2 L_{p}} n_{d_{o p t}+2 L_{p}}^{*}+\left|n_{d_{o p t}+2 L_{p}}\right|^{2}\right)\right\} \\
= & \left(\sigma_{s}^{2}+\sigma_{n}^{2}\right) E\left\{s_{d_{o p t}} s_{d_{o p t}+L_{p}}^{*}\right\} \\
= & e^{-j \phi} \sigma_{s}^{2}\left(\sigma_{s}^{2}+\sigma_{n}^{2}\right)
\end{aligned}
$$

$$
\begin{aligned}
& E\left\{d_{L_{p}}^{*} q_{0}\right\} \\
&=E\left\{\left(s_{d_{o p t}+L_{p}} s_{d_{o p t}+2 L_{p}}^{*}+s_{d_{o p t}+L_{p}} n_{d_{o p t}+2 L_{p}}^{*}+s_{d_{o p t}+2 L_{p}}^{*} n_{d_{o p t}+L_{p}}+n_{d_{o p t}+L_{p}} n_{d_{o p t}+2 L_{p}}^{*}\right)\right. \\
&\left.\quad \cdot\left(\left|s_{d_{o p t}+L_{p}}\right|^{2}+s_{d_{o p t}+L_{p}}^{*} n_{d_{o p t}+L_{p}}+s_{d_{o p t}+L_{p}} n_{d_{o p t}+L_{p}}^{*}+\left|n_{d_{o p t}+L_{p}}\right|^{2}\right)\right\} \\
&=\left(\sigma_{s}^{2}+\sigma_{n}^{2}\right) E\left\{s_{d_{o p t+L_{p}}} s_{d_{o p t}+2 L_{p}}^{*}\right\}+\sigma_{n}^{2} E\left\{s_{d_{o p t}+L_{p}} s_{d_{o p t}+2 L_{p}}^{*}\right\} \\
&= \begin{cases}-e^{-j \phi} \sigma_{s}^{2}\left(\sigma_{s}^{2}+2 \sigma_{n}^{2}\right) & \text { using the FIP preamble. } \\
0 & \text { using SC's preamble. }\end{cases}
\end{aligned}
$$

$$
\begin{aligned}
& E\left\{d_{m}^{*} q_{m}\right\}= \\
& E\left\{\left(s_{d_{o p t}+m} s_{d_{o p t}+m+L_{p}}^{*}+s_{d_{o p t}+m} n_{d_{o p t}+m+L_{p}}^{*}+s_{d_{o p t}+m+L_{p}}^{*} n_{d_{o p t}+m}+n_{d_{o p t}+m} n_{d_{o p t}+m+L_{p}}^{*}\right)\right. \\
& \left.\cdot\left(\left|s_{d_{o p t}+m+L_{p}}\right|^{2}+s_{d_{o p t}+m+L_{p}}^{*} n_{d_{o p t}+m+L_{p}}+s_{d_{o p t}+m+L_{p}} n_{d_{o p t}+m+L_{p}}^{*}+\left|n_{d_{o p t}+m+L_{p}}\right|^{2}\right)\right\} \\
& =\left(\sigma_{s}^{2}+\sigma_{n}^{2}\right) E\left\{s_{d_{o p t}+m} s_{d_{o p t}+m+L_{p}}^{*}\right\}+\sigma_{n}^{2} E\left\{s_{d_{o p t}+m} s_{d_{o p t}+m+L_{p}}^{*}\right\} \\
& = \begin{cases}e^{-j \phi} \sigma_{s}^{2}\left(\sigma_{s}^{2}+2 \sigma_{n}^{2}\right), & m=0,1, \cdots, L_{p}-1 . \\
-e^{-j \phi} \sigma_{s}^{2}\left(\sigma_{s}^{2}+2 \sigma_{n}^{2}\right), & m=L_{p} . \quad \text { using the FlP preamble. } \\
0, & m=L_{p} . \quad \text { using SC's preamble. }\end{cases}
\end{aligned}
$$




$$
\begin{aligned}
E\left\{\left(d_{0}^{*}-d_{L_{p}}^{*}\right)\left(q_{L_{p}}-q_{0}\right)\right\} & =E\left\{d_{0}^{*} q_{L_{p}}-d_{0}^{*} q_{0}-d_{L_{p}}^{*} q_{L_{p}}+d_{L_{p}}^{*} q_{0}\right\} \\
& =E\left\{d_{0}^{*} q_{L_{p}}\right\}-E\left\{d_{0}^{*} q_{0}\right\}-E\left\{d_{L_{p}}^{*} q_{L_{p}}\right\}+E\left\{d_{L_{p}}^{*} q_{0}\right\} \\
& = \begin{cases}-e^{-j \phi} \sigma_{s}^{2} \sigma_{n}^{2} & \text { using the FIP preamble. } \\
-e^{-j \phi} \sigma_{s}^{2} \sigma_{n}^{2} & \text { using SC's preamble. }\end{cases}
\end{aligned}
$$

$$
\begin{aligned}
& E\left\{\sum_{m=1}^{L_{p}-1} d_{m} \sum_{n=1}^{L_{p}-1} q_{n}\right\}=E\left\{\sum_{m=1}^{L_{p}-1} d_{m} q_{m}+\sum_{m=1}^{L_{p}-1} \sum_{n=1, n \neq m}^{L_{p}-1} d_{m} q_{n}\right\} \\
& =\sum_{m=1}^{L_{p}-1} E\left\{d_{m} q_{m}\right\}+\sum_{m=1}^{L_{p}-1} \sum_{n=1, n \neq m}^{L_{p}-1} E\left\{d_{m} q_{n}\right\} \\
& =\sum_{m=1}^{L_{p}-1}\left(E\left\{d_{m}^{*} q_{m}\right\}\right)^{*}+\sum_{m=1}^{L_{p}-1} \sum_{n=1, n \neq m}^{L_{p}-1} \\
& E\left\{\left(s_{d_{o p t}+m}^{*} s_{d_{o p t}+m+L_{p}}+s_{d_{o p t}+m}^{*} n_{d_{o p t}+m+L_{p}}+s_{d_{o p t}+m+L_{p}} n_{d_{o p t}+m}^{*}+n_{d_{o p t}+m}^{*} n_{d_{o p t}+m+L_{p}}\right)\right. \\
& \left.\quad \cdot\left(\left|s_{d_{o p t}+n+L_{p}}\right|^{2}+s_{d_{o p t}+n+L_{p}}^{*} n_{d_{o p t}+n+L_{p}}+s_{d_{o p t}+n+L_{p}} n_{d_{o p t}+n+L_{p}}^{*}+\left|n_{d_{o p t}+n+L_{p}}\right|^{2}\right)\right\} \\
& =\left(L_{p}-1\right) e^{j \phi} \sigma_{s}^{2}\left(\sigma_{s}^{2}+2 \sigma_{n}^{2}\right)+\left(L_{p}-1\right)\left(L_{p}-2\right) e^{j \phi} \sigma_{s}^{2}\left(\sigma_{s}^{2}+\sigma_{n}^{2}\right) \\
& =e^{j \phi}\left[\left(L_{p}-1\right)^{2} \sigma_{s}^{2}\left(\sigma_{s}^{2}+\sigma_{n}^{2}\right)+\left(L_{p}-1\right) \sigma_{s}^{2} \sigma_{n}^{2}\right]
\end{aligned}
$$

$$
\begin{aligned}
& E\left\{\Re\left\{\left(d_{0}^{*}-d_{L_{p}}^{*}\right) \sum_{m=1}^{L_{p}-1} d_{m}\right\}\left(q_{L_{p}}-q_{0}\right) \sum_{n=1}^{L_{p}-1} q_{n}\right\} \\
& =\Re\left\{E\left\{\left(d_{0}^{*}-d_{L_{p}}^{*}\right)\left(q_{L_{p}}-q_{0}\right) \sum_{m=1}^{L_{p}-1} d_{m} \sum_{n=1}^{L_{p}-1} q_{n}\right\}\right\} \\
& =\Re\left\{E\left\{\left(d_{0}^{*}-d_{L_{p}}^{*}\right)\left(q_{L_{p}}-q_{0}\right)\right\} E\left\{\sum_{m=1}^{L_{p}-1} d_{m} \sum_{n=1}^{L_{p}-1} q_{n}\right\}\right\} \\
& =-\sigma_{s}^{2} \sigma_{n}^{2}\left[\left(L_{p}-1\right)^{2} \sigma_{s}^{2}\left(\sigma_{s}^{2}+\sigma_{n}^{2}\right)+\left(L_{p}-1\right) \sigma_{s}^{2} \sigma_{n}^{2}\right]
\end{aligned}
$$




$$
\begin{aligned}
& E\left\{d_{m}^{*} q_{m}^{2}\right\}= \\
& E\left\{\left(s_{d_{o p t}+m} s_{d_{o p t}+m+L_{p}}^{*}+s_{d_{o p t}+m} n_{d_{o p t}+m+L_{p}}^{*}+s_{d_{o p t}+m+L_{p}}^{*} n_{d_{o p t}+m}+n_{d_{o p t}+m} n_{d_{o p t}+m+L_{p}}^{*}\right)\right. \\
& \cdot\left[\left|s_{d_{o p t}+m+L_{p}}\right|^{4}+4\left|s_{d_{o p t}+m+L_{p}}\right|^{2}\left|n_{d_{o p t}+m+L_{p}}\right|^{2}+\left|n_{d_{o p t}+m+L_{p}}\right|^{4}\right. \\
& +2\left(\left|s_{d_{o p t}+m+L_{p}}\right|^{2}+\left|n_{d_{o p t}+m+L_{p}}\right|^{2}\right)\left(s_{d_{o p t}+m+L_{p}}^{*} n_{d_{o p t}+m+L_{p}}+s_{d_{o p t}+m+L_{p}} n_{d_{o p t}+m+L_{p}}^{*}\right) \\
& \left.\left.+\left(s_{d_{o p t}+m+L_{p}}^{*} n_{d_{o p t}+m+L_{p}}\right)^{2}+\left(s_{d_{o p t}+m+L_{p}} n_{d_{o p t}+m+L_{p}}^{*}\right)^{2}\right]\right\} \\
& =\left(\sigma_{s}^{4}+4 \sigma_{s}^{2} \sigma_{n}^{2}+\sigma_{n}^{4}\right) E\left\{s_{d_{o p t}+m} s_{d_{o p t}+m+L_{p}}^{*}\right\}+2 \sigma_{n}^{2}\left(\sigma_{s}^{2}+\sigma_{n}^{2}\right) E\left\{s_{d_{o p t}+m} s_{d_{o p t}+m+L_{p}}^{*}\right\} \\
& =\left\{\begin{array}{lll}
e^{-j \phi} \sigma_{s}^{2}\left(\sigma_{s}^{4}+6 \sigma_{s}^{2} \sigma_{n}^{2}+3 \sigma_{n}^{4}\right), & m=0,1, \cdots, L_{p}-1 . \\
-e^{-j \phi} \sigma_{s}^{2}\left(\sigma_{s}^{4}+6 \sigma_{s}^{2} \sigma_{n}^{2}+3 \sigma_{n}^{4}\right), & m=L_{p} . \quad \text { using the FlP preamble. } \\
0, & m=L_{p} . \quad \text { using SC's preamble. }
\end{array}\right.
\end{aligned}
$$

$$
\begin{aligned}
E\left\{d_{0}^{*} q_{L_{p}}^{2}\right\}=E & \left\{\left(s_{d_{o p t}} s_{d_{o p t}+L_{p}}^{*}+s_{d_{o p t}} n_{d_{o p t}+L_{p}}^{*}+s_{d_{o p t}+L_{p}}^{*} n_{d_{o p t}}+n_{d_{o p t}} n_{d_{o p t}+L_{p}}^{*}\right)\right. \\
& \cdot\left[\left|s_{d_{o p t}+2 L_{p}}\right|^{4}+4\left|s_{d_{o p t}+2 L_{p}}\right|^{2}\left|n_{d_{o p t}+2 L_{p}}\right|^{2}+\left|n_{d_{o p t}+2 L_{p}}\right|^{4}\right. \\
& +2\left(\left|s_{d_{o p t}+2 L_{p}}\right|^{2}+\left|n_{d_{o p t}+2 L_{p}}\right|^{2}\right)\left(s_{d_{o p t}+2 L_{p}}^{*} n_{d_{o p t}+2 L_{p}}+s_{d_{o p t}+2 L_{p}} n_{d_{o p t}+2 L_{p}}^{*}\right) \\
& \left.\left.+\left(s_{d_{o p t}+2 L_{p}}^{*} n_{d_{o p t}+2 L_{p}}\right)^{2}+\left(s_{d_{o p t}+2 L_{p}} n_{d_{o p t}+2 L_{p}}^{*}\right)^{2}\right]\right\} \\
=( & \left.\sigma_{s}^{4}+4 \sigma_{s}^{2} \sigma_{n}^{2}+\sigma_{n}^{4}\right) E\left\{s_{d_{o p t}} s_{d_{o p t}+L_{p}}^{*}\right\}=e^{-j \phi} \sigma_{s}^{2}\left(\sigma_{s}^{4}+4 \sigma_{s}^{2} \sigma_{n}^{2}+\sigma_{n}^{4}\right)
\end{aligned}
$$

$$
\begin{aligned}
& E\left\{d_{L_{p}}^{*} q_{0}^{2}\right\}=E\left\{\left(s_{d_{o p t}+L_{p}} s_{d_{o p t}+2 L_{p}}^{*}+s_{d_{o p t}+L_{p}} n_{d_{o p t}+2 L_{p}}^{*}+s_{d_{o p t}+2 L_{p}}^{*} n_{d_{o p t}+L_{p}}\right.\right. \\
& \left.\quad+n_{d_{o p t}+L_{p}} n_{d_{o p t}+2 L_{p}}^{*}\right) \cdot\left[\left|s_{d_{o p t}+L_{p}}\right|^{4}+4\left|s_{d_{o p t}+L_{p}}\right|^{2}\left|n_{d_{o p t}+L_{p}}\right|^{2}+\left|n_{d_{o p t}+L_{p}}\right|^{4}\right. \\
& \quad+2\left(\left|s_{d_{o p t}+L_{p}}\right|^{2}+\left|n_{d_{o p t}+L_{p}}\right|^{2}\right)\left(s_{d_{o p t}+L_{p}}^{*} n_{d_{o p t}+L_{p}}+s_{d_{o p t}+L_{p}} n_{d_{o p t}+L_{p}}^{*}\right) \\
& \left.\left.\quad+\left(s_{d_{o p t}+L_{p}}^{*} n_{d_{o p t}+L_{p}}\right)^{2}+\left(s_{d_{o p t}+L_{p}} n_{d_{o p t}+L_{p}}^{*}\right)^{2}\right]\right\} \\
& =\left(\sigma_{s}^{4}+4 \sigma_{s}^{2} \sigma_{n}^{2}+\sigma_{n}^{4}\right) E\left\{s_{d_{o p t}+L_{p}} s_{d_{o p t}+2 L_{p}}^{*}\right\}+2 \sigma_{n}^{2}\left(\sigma_{s}^{2}+\sigma_{n}^{2}\right) E\left\{s_{d_{o p t}+L_{p}} s_{d_{o p t}+2 L_{p}}^{*}\right\}
\end{aligned}
$$


$= \begin{cases}-e^{-j \phi} \sigma_{s}^{2}\left(\sigma_{s}^{4}+6 \sigma_{s}^{2} \sigma_{n}^{2}+3 \sigma_{n}^{4}\right) & \text { using the FlP preamble. } \\ 0 & \text { using SC's preamble. }\end{cases}$

$$
\begin{aligned}
E\left\{\left(q_{L_{p}}^{2}-q_{0}^{2}\right)\left(d_{0}^{*}-d_{L_{p}}^{*}\right)\right\} & =E\left\{q_{L_{p}}^{2} d_{0}^{*}-q_{0}^{2} d_{0}^{*}-q_{L_{p}}^{2} d_{L_{p}}^{*}+q_{0}^{2} d_{L_{p}}^{*}\right\} \\
& =E\left\{d_{0}^{*} q_{L_{p}}^{2}\right\}-E\left\{d_{0}^{*} q_{0}^{2}\right\}-E\left\{d_{L_{p}}^{*} q_{L_{p}}^{2}\right\}+E\left\{d_{L_{p}}^{*} q_{0}^{2}\right\} \\
& =-2 e^{-j \phi} \sigma_{s}^{2} \sigma_{n}^{2}\left(\sigma_{s}^{2}+\sigma_{n}^{2}\right)
\end{aligned}
$$

$$
\begin{aligned}
& E\left\{\left(q_{L_{p}}^{2}-q_{0}^{2}\right) \Re\left\{\left(d_{0}^{*}-d_{L_{p}}^{*}\right) \sum_{m=1}^{L_{p}-1} d_{m}\right\}\right\} \\
& =\Re\left\{E\left\{\left(q_{L_{p}}^{2}-q_{0}^{2}\right)\left(d_{0}^{*}-d_{L_{p}}^{*}\right) \sum_{m=1}^{L_{p}-1} d_{m}\right\}\right\} \\
& =\Re\left\{E\left\{\left(q_{L_{p}}^{2}-q_{0}^{2}\right)\left(d_{0}^{*}-d_{L_{p}}^{*}\right)\right\} E\left\{\sum_{m=1}^{L_{p}-1} d_{m}\right\}\right\} \\
& =\Re\left\{\left(-2 e^{-j \phi} \sigma_{s}^{2} \sigma_{n}^{2}\left(\sigma_{s}^{2}+\sigma_{n}^{2}\right)\right)\left(e^{j \phi} \sigma_{s}^{2}\left(L_{p}-1\right)\right)\right\} \\
& =-2\left(L_{p}-1\right) \sigma_{s}^{4} \sigma_{n}^{2}\left(\sigma_{s}^{2}+\sigma_{n}^{2}\right)
\end{aligned}
$$




$$
\begin{aligned}
& E\left\{\left|d_{m}\right|^{2} q_{m}\right\}=E\left\{\left(\left|s_{d_{o p t}+m}\right|^{2}\left|s_{d_{o p t}+m+L_{p}}\right|^{2}+\left|s_{d_{o p t}+m}\right|^{2} n_{d_{o p t}+m+L_{p}}^{*} s_{d_{o p t}+m+L_{p}}\right.\right. \\
& \quad+\left|s_{d_{o p t}+m+L_{p}}\right|^{2} s_{d_{o p t}+m}^{*} n_{d_{o p t}+m}+n_{d_{o p t}+m} n_{d_{o p t}+m+L_{p}}^{*} s_{d_{o p t}+m}^{*} s_{d_{o p t}+m+L_{p}} \\
& \quad+\left|s_{d_{o p t}+m}\right|^{2} s_{d_{o p t}+m+L_{p}}^{*} n_{d_{o p t}+m+L_{p}}+\left|s_{d_{o p t}+m}\right|^{2}\left|n_{d_{o p t}+m+L_{p}}\right|^{2} \\
& \quad+s_{d_{o p t}+m+L_{p}}^{*} n_{d_{o p t}+m} s_{d_{o p t}+m}^{*} n_{d_{o p t}+m+L_{p}}+n_{d_{o p t}+m} s_{d_{o p t}+m}^{*}\left|n_{d_{o p t}+m+L_{p}}\right|^{2} \\
& \quad+s_{d_{o p t}+m}\left|s_{d_{o p t}+m+L_{p}}\right|^{2} n_{d_{o p t}+m}^{*}+s_{d_{o p t}+m} n_{d_{o p t}+m+L_{p}}^{*} s_{d_{o p t}+m+L_{p}} n_{d_{o p t}+m}^{*} \\
& \quad+\left|s_{d_{o p t}+m+L_{p}}\right|^{2}\left|n_{d_{o p t}+m}\right|^{2}+\left|n_{d_{o p t}+m}\right|^{2} n_{d_{o p t}+m+L_{p}}^{*} s_{d_{o p t}+m+L_{p}} \\
& \quad+s_{d_{o p t}+m} s_{d_{o p t}+m+L_{p}}^{*} n_{d_{o p t}+m}^{*} n_{d_{o p t}+m+L_{p}}+s_{d_{o p t}+m} n_{d_{o p t}+m}^{*}\left|n_{d_{o p t}+m+L_{p}}\right|^{2} \\
& \left.\quad+s_{d_{o p t}+m+L_{p}}^{*}\left|n_{d_{o p t}+m}\right|^{2} n_{d_{o p t}+m+L_{p}}+\left|n_{d_{o p t}+m}\right|^{2}\left|n_{d_{o p t}+m+L_{p}}\right|^{2}\right) \\
& \left.\quad\left(\left|s_{d_{o p t}+m+L_{p}}\right|^{2}+s_{d_{o p t}+m+L_{p}}^{*} n_{d_{o p t}+m+L_{p}}+s_{d_{o p t}+m+L_{p}} n_{d_{o p t}+m+L_{p}}^{*}+\left|n_{d_{o p t}+m+L_{p}}\right|^{2}\right)\right\} \\
& =\left(\sigma_{s}^{2}+\sigma_{n}^{2}\right)^{3}+2 \sigma_{s}^{2} \sigma_{n}^{2}\left(\sigma_{s}^{2}+\sigma_{n}^{2}\right)
\end{aligned}
$$

$$
\begin{aligned}
E\left\{\left|d_{0}\right|^{2} q_{L_{p}}\right\}=E\{(\mid & \left.s_{d_{o p t}}\right|^{2}\left|s_{d_{o p t}+L_{p}}\right|^{2}+\left|s_{d_{o p t}}\right|^{2} n_{d_{o p t}+L_{p}}^{*} s_{d_{o p t}+L_{p}} \\
& +\left|s_{d_{o p t}+L_{p}}\right|^{2} s_{d_{o p t}}^{*} n_{d_{o p t}}+n_{d_{o p t}} n_{d_{o p t}+L_{p}}^{*} s_{d_{o p t}}^{*} s_{d_{o p t}+L_{p}} \\
& +\left|s_{d_{o p t}}\right|^{2} s_{d_{o p t}+L_{p}}^{*} n_{d_{o p t}+L_{p}}+\left|s_{d_{o p t}}\right|^{2}\left|n_{d_{o p t}+L_{p}}\right|^{2} \\
& +s_{d_{o p t}+L_{p}}^{*} n_{d_{o p t}} s_{d_{o p t}}^{*} n_{d_{o p t}+L_{p}}+n_{d_{o p t}} s_{d_{o p t}}^{*}\left|n_{d_{o p t}+L_{p}}\right|^{2} \\
& +s_{d_{o p t}}\left|s_{d_{o p t}+L_{p}}\right|^{2} n_{d_{o p t}}^{*}+s_{d_{o p t}} n_{d_{o p t}+L_{p}}^{*} s_{d_{o p t}+L_{p}} n_{d_{o p t}}^{*} \\
& +\left|s_{d_{o p t}+L_{p}}\right|^{2}\left|n_{d_{o p t}}\right|^{2}+\left|n_{d_{o p t}}\right|^{2} n_{d_{o p t}+L_{p}}^{*} s_{d_{o p t}+L_{p}} \\
& +s_{d_{o p t}} s_{d_{o p t}+L_{p}}^{*} n_{d_{o p t}}^{*} n_{d_{o p t}+L_{p}}+s_{d_{o p t}} n_{d_{o p t}}^{*}\left|n_{d_{o p t}+L_{p}}\right|^{2} \\
& \left.+s_{d_{o p t}+L_{p}}^{*}\left|n_{d_{o p t}}\right|^{2} n_{d_{o p t}+L_{p}}+\left|n_{d_{o p t}}\right|^{2}\left|n_{d_{o p t}+L_{p}}\right|^{2}\right) \\
& \left.\cdot\left(\left|s_{d_{o p t}}+2 L_{p}\right|^{2}+s_{d_{o p t}+2 L_{p}}^{*} n_{d_{o p t}+2 L_{p}}+s_{d_{o p t}+2 L_{p}} n_{d_{o p t}+2 L_{p}}^{*}+\left|n_{d_{o p t}+2 L_{p}}\right|^{2}\right)\right\} \\
=\left(\sigma_{s}^{2}+\right. & \left.\sigma_{n}^{2}\right)^{3}
\end{aligned}
$$




$$
\begin{aligned}
& E\left\{\left|d_{L_{p}}\right|^{2} q_{0}\right\}=E\left\{\left(\left|s_{d_{o p t}+L_{p}}\right|^{2}\left|s_{d_{o p t}+2 L_{p}}\right|^{2}+\left|s_{d_{o p t}+L_{p}}\right|^{2} n_{d_{o p t}+2 L_{p}}^{*} s_{d_{o p t}+2 L_{p}}\right.\right. \\
& +\left|s_{d_{o p t}+2 L_{p}}\right|^{2} s_{d_{o p t}+L_{p}}^{*} n_{d_{o p t}+L_{p}}+n_{d_{o p t}+L_{p}} n_{d_{o p t}+2 L_{p}}^{*} s_{d_{o p t}+L_{p}}^{*} s_{d_{o p t}+2 L_{p}} \\
& +\left|s_{d_{o p t}+L_{p}}\right|^{2} s_{d_{o p t}+2 L_{p}}^{*} n_{d_{o p t}+2 L_{p}}+\left|s_{d_{o p t}+L_{p}}\right|^{2}\left|n_{d_{o p t}+2 L_{p}}\right|^{2} \\
& +s_{d_{o p t}+2 L_{p}}^{*} n_{d_{o p t}+L_{p}} s_{d_{o p t}+L_{p}}^{*} n_{d_{o p t}+2 L_{p}}+n_{d_{o p t}+L_{p}} s_{d_{o p t}+L_{p}}^{*}\left|n_{d_{o p t}+2 L_{p}}\right|^{2} \\
& +s_{d_{o p t}+L_{p}}\left|s_{d_{o p t}+2 L_{p}}\right|^{2} n_{d_{o p t}+L_{p}}^{*}+s_{d_{o p t}+L_{p}} n_{d_{o p t}+2 L_{p}}^{*} s_{d_{o p t}+2 L_{p}} n_{d_{o p t}+L_{p}}^{*} \\
& +\left|s_{d_{o p t}+2 L_{p}}\right|^{2}\left|n_{d_{o p t}+L_{p}}\right|^{2}+\left|n_{d_{o p t}+L_{p}}\right|^{2} n_{d_{o p t}+2 L_{p}}^{*} s_{d_{o p t}+2 L_{p}} \\
& +s_{d_{o p t}+L_{p}} s_{d_{o p t}+2 L_{p}}^{*} n_{d_{o p t}+L_{p}}^{*} n_{d_{o p t}+2 L_{p}}+s_{d_{o p t}+L_{p}} n_{d_{o p t}+L_{p}}^{*}\left|n_{d_{o p t}+2 L_{p}}\right|^{2} \\
& \left.+s_{d_{o p t}+2 L_{p}}^{*}\left|n_{d_{o p t}+L_{p}}\right|^{2} n_{d_{o p t}+2 L_{p}}+\left|n_{d_{o p t}+L_{p}}\right|^{2}\left|n_{d_{o p t}+2 L_{p}}\right|^{2}\right) \\
& \left.\cdot\left(\left|s_{d_{o p t}+L_{p}}\right|^{2}+s_{d_{o p t}+L_{p}}^{*} n_{d_{o p t}+L_{p}}+s_{d_{o p t}+L_{p}} n_{d_{o p t}+L_{p}}^{*}+\left|n_{d_{o p t}+L_{p}}\right|^{2}\right)\right\} \\
& =\left(\sigma_{s}^{2}+\sigma_{n}^{2}\right)^{3}+2 \sigma_{s}^{2} \sigma_{n}^{2}\left(\sigma_{s}^{2}+\sigma_{n}^{2}\right) \\
& E\left\{\left(\left|d_{0}\right|^{2}-\left|d_{L_{p}}\right|^{2}\right)\left(q_{L_{p}}-q_{0}\right)\right\}=E\left\{\left|d_{0}\right|^{2} q_{L_{p}}-\left|d_{L_{p}}\right|^{2} q_{L_{p}}-\left|d_{0}\right|^{2} q_{0}+\left|d_{L_{p}}\right|^{2} q_{0}\right\} \\
& =E\left\{\left|d_{0}\right|^{2} q_{L_{p}}\right\}-E\left\{\left|d_{L_{p}}\right|^{2} q_{L_{p}}\right\}-E\left\{\left|d_{0}\right|^{2} q_{0}\right\}+E\left\{\left|d_{L_{p}}\right|^{2} q_{0}\right\} \\
& =\left(\sigma_{s}^{2}+\sigma_{n}^{2}\right)^{3}-2\left[\left(\sigma_{s}^{2}+\sigma_{n}^{2}\right)^{3}+2 \sigma_{s}^{2} \sigma_{n}^{2}\left(\sigma_{s}^{2}+\sigma_{n}^{2}\right)\right]+\left(\sigma_{s}^{2}+\sigma_{n}^{2}\right)^{3}+2 \sigma_{s}^{2} \sigma_{n}^{2}\left(\sigma_{s}^{2}+\sigma_{n}^{2}\right) \\
& =-2 \sigma_{s}^{2} \sigma_{n}^{2}\left(\sigma_{s}^{2}+\sigma_{n}^{2}\right) \\
& E\left\{\left(\left|d_{0}\right|^{2}-\left|d_{L_{p}}\right|^{2}\right)\left(q_{L_{p}}-q_{0}\right) \sum_{n=1}^{L_{p}-1} q_{n}\right\}=E\left\{\left(\left|d_{0}\right|^{2}-\left|d_{L_{p}}\right|^{2}\right)\left(q_{L_{p}}-q_{0}\right)\right\} E\left\{\sum_{n=1}^{L_{p}-1} q_{n}\right\} \\
& =\left(-2 \sigma_{s}^{2} \sigma_{n}^{2}\left(\sigma_{s}^{2}+\sigma_{n}^{2}\right)\right)\left[\left(L_{p}-1\right)\left(\sigma_{s}^{2}+\sigma_{n}^{2}\right)\right] \\
& =-2\left(L_{p}-1\right) \sigma_{s}^{2} \sigma_{n}^{2}\left(\sigma_{s}^{2}+\sigma_{n}^{2}\right)^{2}
\end{aligned}
$$


From (A.63), (A.67), (A.73), (A.78) and (A.83), we have

$$
\begin{aligned}
& \operatorname{Cov}\left(P_{d}, R_{d}\right) \\
& =E\left\{\left(\left|d_{0}\right|^{2}-\left|d_{L_{p}}\right|^{2}\right)\left(q_{L_{p}}^{2}-q_{0}^{2}\right)\right\}+4 E\left\{\Re\left\{\left(d_{0}^{*}-d_{L_{p}}^{*}\right) \sum_{m=1}^{L_{p}-1} d_{m}\right\}\left(q_{L_{p}}-q_{0}\right) \sum_{n=1}^{L_{p}-1} q_{n}\right\} \\
& +2 E\left\{\left(q_{L_{p}}^{2}-q_{0}^{2}\right) \Re\left\{\left(d_{0}^{*}-d_{L_{p}}^{*}\right) \sum_{m=1}^{L_{p}-1} d_{m}\right\}\right\}+2 E\left\{\left(\left|d_{0}\right|^{2}-\left|d_{L_{p}}\right|^{2}\right)\left(q_{L_{p}}-q_{0}\right) \sum_{n=1}^{L_{p}-1} q_{n}\right\} \\
& =\left[-8 \sigma_{s}^{2} \sigma_{n}^{2}\left(\sigma_{s}^{2}+\sigma_{n}^{2}\right)^{2}\right]+4\left[-\sigma_{s}^{2} \sigma_{n}^{2}\left[\left(L_{p}-1\right)^{2} \sigma_{s}^{2}\left(\sigma_{s}^{2}+\sigma_{n}^{2}\right)+\left(L_{p}-1\right) \sigma_{s}^{2} \sigma_{n}^{2}\right]\right] \\
& +2\left[-2\left(L_{p}-1\right) \sigma_{s}^{4} \sigma_{n}^{2}\left(\sigma_{s}^{2}+\sigma_{n}^{2}\right)\right]+2\left[-2\left(L_{p}-1\right) \sigma_{s}^{2} \sigma_{n}^{2}\left(\sigma_{s}^{2}+\sigma_{n}^{2}\right)^{2}\right] \\
& =-4\left[\left(L_{p}-1\right)^{2} \sigma_{s}^{4} \sigma_{n}^{2}\left(\sigma_{s}^{2}+\sigma_{n}^{2}\right)+\left(L_{p}-1\right) \sigma_{s}^{4} \sigma_{n}^{2}\left(\sigma_{s}^{2}+2 \sigma_{n}^{2}\right)+\left(L_{p}+1\right) \sigma_{s}^{2} \sigma_{n}^{2}\left(\sigma_{s}^{2}+\sigma_{n}^{2}\right)^{2}\right]
\end{aligned}
$$

From (A.14), (A.22) and (A.48), we have

$$
E\left\{M_{d}\right\}=c_{1} \cdot E\left\{P_{d}\right\}+c_{2} \cdot E\left\{R_{d}\right\}= \begin{cases}\frac{4\left(L_{p}-1\right) \sigma_{s}^{4}}{L_{p}^{2}\left(\sigma_{s}^{2}+\sigma_{n}^{2}\right)^{2}} & \text { using the FlP preamble. } \\ \frac{2\left(L_{p}-1\right) \sigma_{s}^{4}}{L_{p}^{2}\left(\sigma_{s}^{2}+\sigma_{n}^{2}\right)^{2}} & \text { using SC's preamble. }\end{cases}
$$


From (A.15), (A.42), (A.62) and (A.84), we have

$$
\begin{aligned}
& \operatorname{Var}\left(M_{d}\right)=c_{1}^{2} \cdot \operatorname{Var}\left(P_{d}\right)+c_{2}^{2} \cdot \operatorname{Var}\left(R_{d}\right)+2 \cdot c_{1} \cdot c_{2} \cdot \operatorname{Cov}\left(P_{d}, R_{d}\right) \\
& = \begin{cases}\frac{1}{L_{p}^{4}\left(\sigma_{s}^{2}+\sigma_{n}^{2}\right)^{4}}\left[4\left(L_{p}-1\right)^{2}\left(3 \sigma_{s}^{6} \sigma_{n}^{2}+\sigma_{s}^{4} \sigma_{n}^{4}\right)+4\left(L_{p}-1\right)\left(2 \sigma_{s}^{4}+2 \sigma_{s}^{2} \sigma_{n}^{2}+\sigma_{n}^{4}\right)\left(2 \sigma_{s}^{2} \sigma_{n}^{2}+\sigma_{n}^{4}\right)\right. \\
\left.+8\left(\sigma_{s}^{6} \sigma_{n}^{2}+\sigma_{s}^{4} \sigma_{n}^{4}+\sigma_{s}^{2} \sigma_{n}^{6}\right)\right]+\frac{\sigma_{s}^{8}}{L_{p}^{4}\left(\sigma_{s}^{2}+\sigma_{n}^{2}\right)^{8}}\left[16 L_{p}^{2} \sigma_{s}^{2} \sigma_{n}^{2}\left(\sigma_{s}^{2}+\sigma_{n}^{2}\right)^{2}+\left(32 L_{p}-28\right) \sigma_{s}^{4} \sigma_{n}^{4}\right] \\
-8 \frac{\sigma_{s}^{4}}{L_{p}^{4}\left(\sigma_{s}^{2}+\sigma_{n}^{2}\right)^{6}}\left[\left(L_{p}-1\right)^{2} \sigma_{s}^{4} \sigma_{n}^{2}\left(\sigma_{s}^{2}+\sigma_{n}^{2}\right)+\left(L_{p}-1\right) \sigma_{s}^{4} \sigma_{n}^{2}\left(\sigma_{s}^{2}+2 \sigma_{n}^{2}\right)\right. \\
\left.+\left(L_{p}+1\right) \sigma_{s}^{2} \sigma_{n}^{2}\left(\sigma_{s}^{2}+\sigma_{n}^{2}\right)^{2}\right] & \text { using the FIP preamble. } \\
\frac{1}{L_{p}^{4}\left(\sigma_{s}^{2}+\sigma_{n}^{2}\right)^{4}}\left[\left(L_{p}-1\right)^{2} \sigma_{s}^{4}\left(2 \sigma_{s}^{4}+8 \sigma_{s}^{2} \sigma_{n}^{2}+4 \sigma_{n}^{4}\right)+4\left(L_{p}-1\right)\left[3 \sigma_{s}^{4}\left(\sigma_{s}^{2} \sigma_{n}^{2}+\sigma_{n}^{4}\right)\right.\right. \\
\left.\left.+\left(2 \sigma_{s}^{2} \sigma_{n}^{2}+\sigma_{n}^{4}\right)^{2}\right]+8\left(\sigma_{s}^{6} \sigma_{n}^{2}+\sigma_{s}^{4} \sigma_{n}^{4}+\sigma_{s}^{2} \sigma_{n}^{6}\right)\right]+\frac{\sigma_{s}^{8}}{L_{p}^{4}\left(\sigma_{s}^{2}+\sigma_{n}^{2}\right)^{8}}\left[16 L_{p}^{2} \sigma_{s}^{2} \sigma_{n}^{2}\left(\sigma_{s}^{2}+\sigma_{n}^{2}\right)^{2}\right. \\
\left.+\left(32 L_{p}-28\right) \sigma_{s}^{4} \sigma_{n}^{4}\right]-8 \frac{\sigma_{s}^{4}}{L_{p}^{4}\left(\sigma_{s}^{2}+\sigma_{n}^{2}\right)^{6}}\left[\left(L_{p}-1\right)^{2} \sigma_{s}^{4} \sigma_{n}^{2}\left(\sigma_{s}^{2}+\sigma_{n}^{2}\right)+\left(L_{p}-1\right) \sigma_{s}^{4} \sigma_{n}^{2}\right. \\
\left.\left(\sigma_{s}^{2}+2 \sigma_{n}^{2}\right)+\left(L_{p}+1\right) \sigma_{s}^{2} \sigma_{n}^{2}\left(\sigma_{s}^{2}+\sigma_{n}^{2}\right)^{2}\right] & \text { using SC's preamble. }\end{cases}
\end{aligned}
$$

When $L_{p}$ is large, $\operatorname{Var}\left(M_{d}\right)$ can be approximated by

$$
\begin{aligned}
& \operatorname{Var}\left(M_{d}\right)=c_{1}^{2} \cdot \operatorname{Var}\left(P_{d}\right)+c_{2}^{2} \cdot \operatorname{Var}\left(R_{d}\right)+2 \cdot c_{1} \cdot c_{2} \cdot \operatorname{Cov}\left(P_{d}, R_{d}\right) \\
& \approx\left\{\begin{array}{l}
\frac{1}{L_{p}^{4}\left(\sigma_{s}^{2}+\sigma_{n}^{2}\right)^{4}}\left[4\left(L_{p}-1\right)^{2}\left(3 \sigma_{s}^{6} \sigma_{n}^{2}+\sigma_{s}^{4} \sigma_{n}^{4}\right)\right]+\frac{\sigma_{s}^{8}}{L_{p}^{4}\left(\sigma_{s}^{2}+\sigma_{n}^{2}\right)^{8}}\left[16 L_{p}^{2} \sigma_{s}^{2} \sigma_{n}^{2}\left(\sigma_{s}^{2}+\sigma_{n}^{2}\right)^{2}\right] \\
-8 \frac{\sigma_{s}^{4}}{L_{p}^{4}\left(\sigma_{s}^{2}+\sigma_{n}^{2}\right)^{6}}\left[\left(L_{p}-1\right)^{2} \sigma_{s}^{4} \sigma_{n}^{2}\left(\sigma_{s}^{2}+\sigma_{n}^{2}\right)\right] \\
\text { using the FlP preamble. } \\
\frac{1}{L_{p}^{4}\left(\sigma_{s}^{2}+\sigma_{n}^{2}\right)^{4}}\left[\left(L_{p}-1\right)^{2} \sigma_{s}^{4}\left(2 \sigma_{s}^{4}+8 \sigma_{s}^{2} \sigma_{n}^{2}+4 \sigma_{n}^{4}\right)\right]+\frac{\sigma_{s}^{8}}{L_{p}^{4}\left(\sigma_{s}^{2}+\sigma_{n}^{2}\right)^{8}}\left[16 L_{p}^{2} \sigma_{s}^{2} \sigma_{n}^{2}\left(\sigma_{s}^{2}+\sigma_{n}^{2}\right)^{2}\right] \\
-8 \frac{\sigma_{s}^{4}}{L_{p}^{4}\left(\sigma_{s}^{2}+\sigma_{n}^{2}\right)^{6}}\left[\left(L_{p}-1\right)^{2} \sigma_{s}^{4} \sigma_{n}^{2}\left(\sigma_{s}^{2}+\sigma_{n}^{2}\right)\right] \\
\text { using SC's preamble. }
\end{array}\right.
\end{aligned}
$$


From (A.85), (A.86) and (A.87), we can see that using the FlP preamble will increase the mean of $M_{d}=M_{p}\left(d_{o p t}\right)-M_{p}\left(d_{o p t+1}\right)$ and decrease the variance of $M_{d}=$ $M_{p}\left(d_{o p t}\right)-M_{p}\left(d_{o p t+1}\right) \quad($ for variance, when $S N R>3 d B)$. Since $M_{d}=M_{p}\left(d_{o p t}\right)-$ $M_{p}\left(d_{\text {opt }+1}\right)$ is approximated by a Gaussian RV, using the FlP preamble will decrease the probability of the start point shifting to the right side. 


\section{Appendix B}

\section{Setting the Threshold for Fine}

\section{Time Synchronization}

Fine time synchronization calculates the cross-correlation function of the frequency

corrected sequences $\left\{r_{k}^{c}\right\}$ with the second transmitted training sequences $\left\{b_{k}\right\}$. Let us assume a perfect CFO estimation. After CFO correction, from (2.49), (2.50) and (4.13), we have

$$
r_{k}^{c}=\left(\sum_{m=0}^{M-1} h_{m} x_{k-n_{\epsilon}-m}\right) e^{j \theta_{0}}+w_{k}
$$

where $M$ is channel length, $h_{m}$ is the channel impulse response, and $w_{k}$ is the complex Gaussian noise with zero mean and variance $\sigma_{n}^{2}$. $\theta_{0}$ is the initial phase for each symbol. Since we assume $\theta_{0}$ does not change in one symbol period and in this thesis the crosscorrelation for fine time synchronization uses only the second training symbol, we can set $\theta_{0}=0$. For simplicity, we also assume $n_{\epsilon}=0$. We have

$$
r_{k}^{c}=\sum_{m=0}^{M-1} h_{m} x_{k-m}+w_{k}
$$


where $x_{k}$ is the $k^{\text {th }}$ sample of the complex transmitted sequences with mean power $\sigma_{x}^{2}$. We have

$$
\begin{gathered}
E\left[\Re\left\{x_{k}\right\}^{2}\right]=E\left[\Im\left\{x_{k}\right\}^{2}\right]=\frac{\sigma_{x}^{2}}{2} \\
E\left[\Re\left\{w_{k}\right\}^{2}\right]=E\left[\Im\left\{w_{k}\right\}^{2}\right]=\frac{\sigma_{n}^{2}}{2}
\end{gathered}
$$

We assume $h_{m}$ is uncorrelated with $x_{k}$ and the $x_{k}$ 's are uncorrelated.

In [6], the author uses the value of $M\left(d_{\text {opt }}\right)$ to estimate the SNR.

$$
\widehat{S N R}=\frac{\sqrt{M\left(d_{\text {opt }}\right)}}{1-\sqrt{M\left(d_{\text {opt }}\right)}}
$$

where $M(d)$ is the timing metric given by (3.4) and $d_{o p t}$ is the exact start point of first training symbol excluding prefix and postfix (Since we do not know the exact start point, we can use the estimated start point to estimate the SNR). Let $d_{\text {opt } 2}$ be the exact start point of the second training symbol excluding CP. From the preamble structure, we have $d_{\text {opt } 2}=d_{\text {opt }}+G / 2+N+G$. Since the proposed method uses similar timing metric as SC's method, we use the estimated coarse time point instead of $d_{\text {opt }}$ and the estimated SNR is

$$
\widehat{S N R}=\frac{\sqrt{M_{p}\left(\widehat{d}_{\text {coarse }}\right)}}{1-\sqrt{M_{p}\left(\widehat{d}_{\text {coarse }}\right)}}
$$

where $M_{p}$ is given by (4.3).

Recall from appendix A (see A.10)) that $R(d)$ is a Gaussian RV with mean $N\left(\sigma_{s}^{2}+\right.$ $\left.\sigma_{n}^{2}\right) / 2$, where $\sigma_{s}^{2}$ is defined in (A.4) and its variance is quite small compared to its mean. $R_{f}(d)$ in (3.12) also has the same mean given by $N\left(\sigma_{s}^{2}+\sigma_{n}^{2}\right) / 2$.

$$
E\{R(d)\}=E\left\{R_{f}(d)\right\}=N\left(\sigma_{s}^{2}+\sigma_{n}^{2}\right) / 2
$$


Recall the cross-correlation in (4.14), $\psi_{n}=\sum_{k=0}^{N-1} b_{k}^{*} r_{n+k}^{c}$. Recall from section 4.1.2, $\widehat{d}_{\text {fine }}=\widehat{n}-N-1.5 G$, where $\widehat{n}$ is the first point in $\left[-G / 2+\widehat{d}_{\text {coarse }}+N+1.5 G, \widehat{d}_{\text {coarse }}+\right.$ $N+1.5 G+G / 2]$ (from smallest to greatest) whose $\left|\psi_{\widehat{n}}\right|$ is greater than a threshold $\eta$. In the following we will indicate how to set to set the threshold for various channels.

- Under AWGN channels

If $M=1$ and $h_{0}=1$, we have $r_{k}^{c}=x_{k}+w_{k}$ and the channel is an AWGN channel. SNR is

$$
S N R=\frac{\sigma_{x}^{2}}{\sigma_{n}^{2}}
$$

Since $x_{d_{o p t 2}+k}=b_{k}$, we have

$$
\psi_{d_{o p t 2}}=\sum_{k=0}^{N-1} b_{k}^{*} r_{d_{o p t 2}+k}^{c}=\sum_{k=0}^{N-1}\left|b_{k}\right|^{2}+\sum_{k=0}^{N-1} b_{k}^{*} w_{d_{o p t 2}+k}
$$

$b_{k}$ is a Chu sequence, we assume the $b_{k}$ 's are uncorrelated and $b_{k}$ is uncorrelated with $w_{k}$.

$$
\begin{aligned}
E\left\{\psi_{d_{o p t 2}}\right\} & =N \sigma_{x}^{2} \\
\operatorname{Var}\left\{\psi_{d_{o p t 2}}\right\} & =E\left\{\psi_{d_{o p t 2}} \psi_{d_{o p t 2}}^{*}\right\}-\left|E\left\{\psi_{d_{o p t 2}}\right\}\right|^{2}=N \sigma_{x}^{2} \sigma_{n}^{2}
\end{aligned}
$$

For $n \neq d_{\text {opt2 }}$, since we search in a small range, we assume $n$ is in the CP of the second symbol. The cross-correlation of $\left\{b_{k}\right\}$ and its circular shift version is zero.

$$
\psi_{n, n \neq d_{o p t 2}}=\sum_{k=0}^{N-1} b_{k}^{*} w_{n+k, n \neq d_{o p t 2}}
$$

we have

$$
E\left\{\psi_{n, n \neq d_{o p t 2}}\right\}=0
$$




$$
\operatorname{Var}\left\{\psi_{n, n \neq d_{\text {opt } 2}}\right\}=N \sigma_{x}^{2} \sigma_{n}^{2}
$$

Since $\sigma_{s}^{2}=\sigma_{x}^{2}$, from (B.6) and (B.7) and using $R_{f}\left(\widehat{d}_{c o a r s e}\right) \approx N\left(\sigma_{s}^{2}+\sigma_{n}^{2}\right) / 2$, we have

$$
\sigma_{s}^{2}=\sigma_{x}^{2} \approx \frac{2 R_{f}\left(\widehat{d}_{c o a r s e}\right) \sqrt{M_{p}\left(\widehat{d}_{c o a r s e}\right)}}{N}
$$

For AWGN channels, we propose to set the threshold

$$
\eta_{A W G N}=0.5 E\left\{\psi_{d_{o p t 2}}\right\}=0.5 N \sigma_{x}^{2} \approx R_{f}\left(\widehat{d}_{c o a r s e}\right) \sqrt{M_{p}\left(\widehat{d}_{c o a r s e}\right)}
$$

From the central limit theorem (CLT), $\psi_{n}$ is a Gaussian RV. The probability of $\left|\psi_{d_{o p t 2}}\right|<\eta_{A W G N}$ and $\left|\psi_{n, n \neq d_{\text {opt }}}\right|>\eta_{A W G N}$ for different SNR is given in Table B.1. Table B.1 shows that the probabilities of missing detection and false

Table B.1 The probability of $\psi_{d_{o p t 2}}<\eta_{A W G N}$ and $\psi_{n, n \neq d_{o p t 2}}>\eta_{A W G N}$ for different SNR under AWGN channels

\begin{tabular}{lllll}
\hline $\mathrm{SNR}(\mathrm{dB})$ & -10 & -5 & 0 & 5 \\
\hline$\left|\psi_{\text {opt }_{2}}\right|<\eta_{A W G N}$ & $2.100 \times 10^{-7}$ & $1.155 \times 10^{-19}$ & $6.389 \times 10^{-58}$ & $2.271 \times 10^{-178}$ \\
\hline & & & & \\
$\left|\psi_{n, n \neq d_{\text {opt } 2}}\right|>\eta_{A W G N}$ & $2.100 \times 10^{-7}$ & $1.155 \times 10^{-19}$ & $6.389 \times 10^{-58}$ & $2.271 \times 10^{-178}$ \\
\hline
\end{tabular}

alarm are quite small for the proposed choice of threshold.

- Under ISI channels

For ISI channels, we use the tapped delay line model given in section 2.1.4, but for each channel tap $h_{m}$, the amplitude of $h_{m}$ is fixed and the phase of $h_{m}$ is fixed or a unform distribution in $[0,2 \pi)$. Recall the frequency corrected samples 
$r_{k}^{c}$ given in (B.2). We normalized the channel power

$$
\sigma_{h}^{2}=\sum_{m=0}^{M-1}\left|h_{m}\right|^{2}=1
$$

The average SNR at the receiver is

$$
S N R=\frac{E\left\{\left|\sum_{m=0}^{M-1} h_{m} x_{k-m}\right|^{2}\right\}}{E\left\{\left|w_{k}\right|^{2}\right\}}=\frac{\left(\sum_{m=0}^{M-1}\left|h_{m}\right|^{2}\right) \sigma_{x}^{2}}{\sigma_{n}^{2}}=\frac{\sigma_{x}^{2}}{\sigma_{n}^{2}}
$$

Since we use Chu sequences as the second training symbol, $b_{k}$ has a perfect autocorrelation function.

$$
\begin{aligned}
\psi_{d_{o p t 2}} & =\sum_{k=0}^{N-1} b_{k}^{*} r_{d_{o p t 2}+k}^{c}=\sum_{k=0}^{N-1} b_{k}^{*} \sum_{m=0}^{l-1} h_{m} x_{d_{o p t 2}+k-m}+\sum_{k=0}^{N-1} b_{k}^{*} w_{d_{o p t 2}+k} \\
& =h_{0} \sum_{k=0}^{N-1}\left|b_{k}\right|^{2}+\sum_{k=0}^{N-1} b_{k}^{*} w_{d_{o p t 2}+k}
\end{aligned}
$$

We have

$$
\begin{gathered}
E\left\{\psi_{d_{o p t 2}}\right\}=h_{0} N \sigma_{x}^{2} \\
\operatorname{Var}\left\{\psi_{d_{o p t 2}}\right\}=E\left\{\psi_{d_{o p t 2}} \psi_{d_{o p t 2}}^{*}\right\}-\left|E\left\{\psi_{d_{o p t 2}}\right\}\right|^{2}=N \sigma_{x}^{2} \sigma_{n}^{2}
\end{gathered}
$$

For $n<d_{\text {opt } 2}$, which means $n$ is on the left side of the exact start point, similar to AWGN channels, we have

$$
\begin{gathered}
\psi_{n, n<d_{o p t 2}}=\sum_{k=0}^{N-1} b_{k}^{*} w_{n+k, n<d_{o p t 2}} \\
E\left\{\psi_{n, n<d_{o p t 2}}\right\}=0 \\
\operatorname{Var}\left\{\psi_{n, n<d_{o p t 2}}\right\}=N \sigma_{x}^{2} \sigma_{n}^{2}
\end{gathered}
$$


In most scenarios, the first tap power of ISI channels would be greater than $-14 \mathrm{~dB}$, which means $\left|h_{0}\right|>0.2$, so setting the threshold corresponding to half the mean of $\psi_{d_{o p t 2}}$ assuming that $h_{0}=0.2$ is reasonable i.e.

$$
\eta_{I S I}=0.5 E\left\{\psi_{d_{\text {opt }}}\right\}=0.5 h_{0} N \sigma_{x}^{2}=0.1 N \sigma_{x}^{2} \approx 0.2 R_{f}\left(\widehat{d}_{\text {coarse }}\right) \sqrt{M_{p}\left(\widehat{d}_{\text {coarse }}\right)}
$$

From the central limit theorem (CLT), $\psi_{n}$ is a Gaussian RV. The probability of $\left|\psi_{d_{o p t} 2}\right|<\eta_{I S I}$ and $\left|\psi_{n, n \neq d_{\text {opt }}}\right|>\eta_{I S I}$ for different SNR is given in Table B.2. Two ISI channels are considered in this thesis with first tap gain $\left|h_{0}\right|=0.37629$. Therefore, Table B. 2 also provide probabilities for a threshold set to half the mean of $\psi_{d_{o p t 2}}$ considering the true $\left|h_{0}\right|$, e.g.

$$
\eta_{1}=0.5 E\left\{\psi_{d_{o p t 2}}\right\}=0.5 h_{0} N \sigma_{x}^{2} \approx 0.19 N \sigma_{x}^{2}
$$

Table B.2 shows that if we choose the threshold to be equal to or little greater

Table B.2 The probability of $\psi_{d_{o p t 2}}<\eta_{I S I}$ and $\psi_{n, n<d_{o p t 2}}>\eta_{I S I}$ for different SNR under ISI channels

\begin{tabular}{lllll}
\hline $\mathrm{SNR}(\mathrm{dB})$ & 0 & 5 & 10 & 15 \\
\hline$\left|\psi_{d_{\text {opt }}}\right|<\eta_{I S I}$ & $4.731 \times 10^{-19}$ & $5.322 \times 10^{-56}$ & $2.592 \times 10^{-172}$ & 0 \\
\hline$\left|\psi_{n, n<d_{\text {opt } 2}}\right|>\eta_{I S I}$ & 0.00068714 & $6.334 \times 10^{-9}$ & $2.269 \times 10^{-24}$ & $1.068 \times 10^{-72}$ \\
\hline \hline$\left|\psi_{d_{\text {opt } 2}}\right|<\eta_{1}$ & $1.251 \times 10^{-9}$ & $1.477 \times 10^{-26}$ & $1.436 \times 10^{-79}$ & $1.124 \times 10^{-246}$ \\
\hline$\left|\psi_{n, n<d_{\text {opt } 2}}\right|>\eta_{1}$ & $6.009 \times 10^{-10}$ & $1.511 \times 10^{-27}$ & $1.107 \times 10^{-82}$ & $1.682 \times 10^{-256}$ \\
\hline
\end{tabular}

than the half of mean of $\psi_{d_{o p t 2}}$, the probability of missing detection and false 
alarm will be at the same level, but we have to know the channel power of the first tap.

From the analysis, to get better performance, the threshold should depend on the SNR. Since we can estimate SNR from the peak value of the timing metric, we can set up a look up table depending on the estimated SNR for setting the threshold. For example, in the simulations presented in this thesis, the chosen threshold for ISI channels is

$$
\eta_{I S I}= \begin{cases}0.2 R_{f}\left(\widehat{d}_{\text {coarse }}\right) \sqrt{M_{p}\left(\widehat{d}_{\text {coarse }}\right)} & \text { if } \widehat{S N R}>5 d B \\ 0.3 R_{f}\left(\widehat{d}_{\text {coarse }}\right) \sqrt{M_{p}\left(\widehat{d}_{\text {coarse }}\right)} & \text { if } \widehat{S N R} \leq 5 d B\end{cases}
$$

- Under Rayleigh fading channels

For the Rayleigh fading channel, each tap amplitude is Rayleigh distributed, and we normalize the mean power of the channel to be one.

$$
E\left\{\sigma_{h}^{2}\right\}=E\left\{\sum_{m=0}^{M-1}\left|h_{m}\right|^{2}\right\}=1
$$

The average SNR at the receiver is

$$
S N R=\frac{E\left\{\left|\sum_{m=0}^{M-1} h_{m} x_{k-m}\right|^{2}\right\}}{E\left\{\left|w_{k}\right|^{2}\right\}}=\frac{E\left\{\sum_{m=0}^{M-1}\left|h_{m}\right|^{2}\right\} \sigma_{x}^{2}}{\sigma_{n}^{2}}=\frac{\sigma_{x}^{2}}{\sigma_{n}^{2}}
$$

Similar to ISI channels

$$
\begin{aligned}
\psi_{d_{o p t 2}} & =\sum_{k=0}^{N-1} b_{k}^{*} r_{d_{o p t 2}+k}^{c}=\sum_{k=0}^{N-1} b_{k}^{*} \sum_{m=0}^{M-1} h_{m} x_{d_{o p t 2}+k-m}+\sum_{k=0}^{N-1} b_{k}^{*} w_{d_{o p t 2}+k} \\
& =h_{0} \sum_{k=0}^{N-1}\left|b_{k}\right|^{2}+\sum_{k=0}^{N-1} b_{k}^{*} w_{d_{o p t 2}+k}
\end{aligned}
$$


We have

$$
\begin{aligned}
E\left\{\psi_{d_{o p t 2}}\right\} & =h_{0} N \sigma_{x}^{2} \\
\operatorname{Var}\left\{\psi_{d_{o p t 2}}\right\} & =E\left\{\psi_{d_{o p t 2}} \psi_{d_{o p t 2}}^{*}\right\}-\left|E\left\{\psi_{d_{o p t 2}}\right\}\right|^{2}=N \sigma_{x}^{2} \sigma_{n}^{2}
\end{aligned}
$$

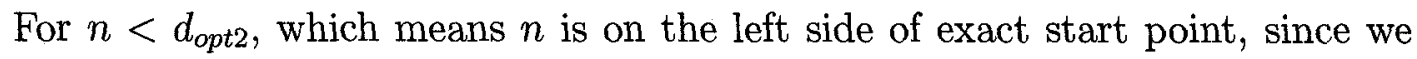
use Chu sequences, we have

$$
\begin{gathered}
\psi_{n, n<d_{o p t 2}}=\sum_{k=0}^{N-1} b_{k}^{*} w_{n+k, n<d_{o p t 2}} \\
E\left\{\psi_{n, n<d_{o p t 2}}\right\}=0 \\
\operatorname{Var}\left\{\psi_{n, n<d_{o p t 2}}\right\}=N \sigma_{x}^{2} \sigma_{n}^{2}
\end{gathered}
$$

Since $\left|h_{0}\right|$ is Rayleigh distributed, sometimes $\left|h_{0}\right|$ might be very small. If we set the threshold too small, the probability of the false alarm would be high and the fine time synchronization would be useless. On the other hand, if the first tap is too weak, we can ignore that tap and choose the first strong tap to be the first tap, which would not result in too much performance loss. Assuming that the first tap power is greater than $-14 \mathrm{~dB}$ (or equivalently $\left|h_{0}\right|>0.2$ ), we can set the threshold for Rayleigh fading channels as

$$
\begin{gathered}
\eta_{\text {Rayleigh }}=0.5 E\left\{\psi_{d_{\text {opt } 2}}\right\}=0.1 N \sigma_{x}^{2} \approx 0.2 R_{f}\left(\widehat{d}_{\text {coarse }}\right) \sqrt{M_{p}\left(\widehat{d}_{\text {coarse }}\right)} \\
\eta_{\text {Rayleigh }}=0.5 E\left\{\psi_{d_{\text {opt } 2}}\right\}=0.1 N \sigma_{x}^{2} \approx 0.2 R_{f}\left(\widehat{d}_{\text {coarse }}\right) \sqrt{M_{p}\left(\widehat{d}_{\text {coarse }}\right)}
\end{gathered}
$$

Similar results as ISI channels would be obtained.

Similar to ISI channels, better performance can be obtained by choosing the thresh- 
old according to SNR. Since we can estimate SNR from the peak value of the timing metric, we can set up a look up table depending on the estimated SNR for setting the threshold. If we know the channel power delay profile, it is very helpful for setting the threshold. For example, in the simulations presented in this thesis, to obtain improved performance, while maintaining reasonable complexity for the look-up table, we propose to set the threshold for Rayleigh as

$$
\eta_{\text {Rayleigh }}= \begin{cases}0.2 R_{f}\left(\widehat{d}_{\text {coarse }}\right) \sqrt{M_{p}\left(\widehat{d}_{\text {coarse }}\right)} & \text { if } \widehat{S N R}>5 d B \\ 0.3 R_{f}\left(\widehat{d}_{\text {coarse }}\right) \sqrt{M_{p}\left(\widehat{d}_{\text {coarse }}\right)} & \text { if } \widehat{S N R} \leq 5 d B\end{cases}
$$




\section{References}

[1] R. V. Nee and R. Prasad, OFDM for wireless multimedia communication. Boston: Artech House, 2000.

[2] T. S. Rappaport, A. Annamalai, R. M. Buehrer, and W. H. Tranter, "Wireless communications: past events and a future perspective," IEEE Communications Magazine, vol. 40, pp. 148 - 161, May 2002.

[3] M. Jankiraman, Space-time codes and MIMO systems. Boston: Artech House, 2004.

[4] W. D. Warner and C. Leung, "OFDM/FM frame synchronization for mobile radio data communication," IEEE Trans. Vehicular Technology, vol. 42, pp. 302 - 313, Aug. 1993.

[5] J. J. van de Beek, M. Sandell, and P. O. Borjesson, "ML estimation of time and frequency offset in OFDM systems," IEEE Trans. Signal Processing, vol. 45, pp. 1800 - 1805, July 1997.

[6] T. M. Schmidl and D. C. Cox, "Robust frequency and timing synchronization for OFDM," IEEE Trans. Communications, vol. 45, pp. 1613 - 1621, Dec. 1997.

[7] H. Minn, M. Zeng, and V. K. Bhargava, "On timing offset estimation for OFDM systems," Communications Letters, IEEE, vol. 4, pp. 242 - 244, July 2000.

[8] K. Shi and E. Serpedin, "Coarse frame and carrier synchronization of OFDM systems: a new metric and comparison," IEEE Trans. Wireless Communications, vol. 3, pp. 1271- 1284, July 2004.

[9] B. Park, H. Cheon, C. Kang, and D. Hong, "A novel timing estimation method for OFDM systems," Communications Letters, IEEE, vol. 7, pp. 239-241, May 2003.

[10] G. Ren, Y. Chang, H. Zhang, and H. Zhang, "Synchronization method based on a new constant envelop preamble for OFDM systems," Broadcasting, IEEE Transactions on, vol. 51, pp. 139- 143, Mar. 2005. 
[11] H. Bolcskei, "MIMO-OFDM wireless systems: basics, perspectives, and challenges," IEEE Trans. Wireless Communications, vol. 13, pp. 31 - 37, Aug. 2006.

[12] M. Schellmann, V. Jungnickel, and C. von Helmolt, "On the value of spatial diversity for the synchronisation in MIMO-OFDM systems," Personal, Indoor and Mobile Radio Communications, 2005. PIMRC 2005. IEEE 16th International Symposium on, vol. 1, pp. 201 - 205, Sept. 2005.

[13] G. L. Stuber, J. R. Barry, S. W. McLaughlin, Y. Li, M. A. Ingram, and T. G. Pratt, "Broadband MIMO-OFDM wireless communications," Proceedings of the IEEE, vol. 92, pp. 271 - 294, Feb. 2004.

[14] A. N. Mody and G. L. Stuber, "Synchronization for MIMO OFDM systems," Global Telecommunications Conference, 2001. GLOBECOM '01. IEEE, vol. 1, pp. $509-513$, Nov. 2001.

[15] A. N. Mody and G. L. Stuber, "Receiver implementation for a MIMO OFDM system," Global Telecommunications Conference, 2002. GLOBECOM '02. IEEE, vol. 1, pp. 716 - 720, Nov. 2002.

[16] Y. Li, J. Cimini, L. J., and N. R. Sollenberger, "Robust channel estimation for OFDM systems with rapid dispersive fading channels," IEEE Trans. Communications, vol. 46, pp. $902-915$, July 1998.

[17] J. G. Proakis, Digital Communications Fourth Edition. Mc Graw Hill, 2000.

[18] T. S. . Rappaport, Wireless communications principles and practice, Second Edition. Prentice Hall, 2001.

[19] S. Weinstein and P. Ebert, "Data transmission by frequency-division multiplexing using the discrete fourier transform," IEEE Trans. Communications, vol. 19, pp. 628 - 634, Oct. 1971.

[20] Y. Li and G. L.Stuber, Orthogonal frequency division multiplexing for wireless communications. New York: Springer, 2006.

[21] C. Wang and Z. Zhou, "A new detection algorithm for OFDM system without cyclic prefix," Emerging Technologies: Frontiers of Mobile and Wireless Communication, 2004. Proceedings of the IEEE 6th Circuits and Systems Symposium on, vol. 2, pp. 453 - 456, May 2004.

[22] J. D. Bakker, "Eliminating the OFDM cyclic prefix," Personal, Indoor and Mobile Radio Communications, 2002. The 13th IEEE International Symposium on, vol. 2, pp. $834-837$, Sept. 2002. 
[23] S. H. Han and J. H. Lee, "An overview of peak-to-average power ratio reduction techniques for multicarrier transmission," IEEE Trans. Communications, vol. 10, pp. $56-65$, Apr. 2005.

[24] A. Czylwik, "Synchronization for systems with antenna diversity," Vehicular Technology Conference, 1999. VTC 1999 - Fall. IEEE VTS 50th, vol. 2, pp. 728 - 732, Sept. 1999.

[25] D. Chu, "Polyphase codes with good periodic correlation properties," IEEE Trans. Inform. Theory, vol. 18, pp. 531 - 532, July 1972.

[26] S. K. Mitra, Digital signal processing : a computer based approach. Boston: Mc Graw Hill, 2001.

[27] W. C. Jakes, Microwave mobile communications. New York: Wiley, 1974.

[28] P. Dent, G. Bottomley, and T. Croft, "Jakes fading model revisited," Electronics Letters, vol. 29, pp. 1162 - 1163, June 1993.

[29] Y. Wen and F. Danilo-Lemoine., "A novel postfix synchronization method for OFDM systems," Accepted by Personal, Indoor and Mobile Radio Communications, 2007. PIMRC 2007. IEEE 18th International Symposium on, Sept. 2007.

[30] E. Zimmermann and et al., "Assessment of radio-link technologies," WINNER Deliverable D2.3, Available: https://www.istwinner. org/DeliverableDocuments/D2.3.pdf, Feb. 2005. 